\title{
Maternal Diet during Pregnancy and Lactation and Risk of Child Food Allergies and Atopic Allergic Diseases: A Systematic Review
}

\author{
2020 Dietary Guidelines Advisory Committee, \\ Pregnancy and Lactation Subcommittee \\ Published date: July 15, 2020
}

Nutrition Evidence Systematic Review

Center for Nutrition Policy and Promotion

Food and Nutrition Service

U.S. Department of Agriculture

Braddock Metro Center II

1320 Braddock Place

Alexandria, Virginia 22314 
This systematic review was conducted by the 2020 Dietary Guidelines Advisory Committee in collaboration with the Nutrition Evidence Systematic Review (NESR) team at the Center for Nutrition Policy and Promotion, Food and Nutrition Service, U.S. Department of Agriculture (USDA). All systematic reviews from the 2020 Advisory Committee Project are available on the NESR website: https://nesr.usda.gov/2020-dietaryguidelines-advisory-committee-systematic-reviews.

Conclusion statements drawn as part of this systematic review describe the state of science related to the specific question examined. Conclusion statements do not draw implications, and should not be interpreted as dietary guidance. This portfolio provides the complete documentation for this systematic review. A summary of this review is included in the 2020 Advisory Committee's Scientific Report available at www.DietaryGuidelines.gov.

The contents of this document may be used and reprinted without permission. Endorsements by NESR, the Center for Nutrition Policy and Promotion, the Food and Nutrition Service, or the USDA of derivative products developed from this work may not be stated or implied.

Suggested citation for this systematic review: 2020 Dietary Guidelines Advisory Committee and Nutrition Evidence Systematic Review Team. Maternal Diet during Pregnancy and Lactation and Risk of Child Food Allergies and Atopic Allergic Diseases: A Systematic Review. 2020 Dietary Guidelines Advisory Committee Project. Alexandria, VA: U.S. Department of Agriculture, Food and Nutrition Service, Center for Nutrition Policy and Promotion, July 2020. Available at: https://nesr.usda.gov/2020-dietary-guidelines-advisorycommittee-systematic-reviews.

Related citation: Dietary Guidelines Advisory Committee. 2020. Scientific Report of the 2020 Dietary Guidelines Advisory Committee: Advisory Report to the Secretary of Agriculture and the Secretary of Health and Human Services. U.S. Department of Agriculture, Agricultural Research Service, Washington, DC.

In accordance with Federal civil rights law and USDA civil rights regulations and policies, the USDA, its Agencies, offices, and employees, and institutions participating in or administering USDA programs are prohibited from discriminating based on race, color, national origin, religion, sex, gender identity (including gender expression), sexual orientation, disability, age, marital status, family/parental status, income derived from a public assistance program, political beliefs, or reprisal or retaliation for prior civil rights activity, in any program or activity conducted or funded by USDA (not all bases apply to all programs). Remedies and complaint filing deadlines vary by program or incident.

Persons with disabilities who require alternative means of communication for program information (e.g., Braille, large print, audiotape, American Sign Language, etc.) should contact the responsible Agency or USDA's TARGET Center at (202) 720-2600 (voice and TTY) or contact USDA through the Federal Relay Service at (800) 877-8339. Additionally, program information may be made available in languages other than English.

To file a program discrimination complaint, complete the USDA Program Discrimination Complaint Form, AD3027, found online at How to File a Program Discrimination Complaint and at any USDA office or write a letter addressed to USDA and provide in the letter all of the information requested in the form. To request a copy of the complaint form, call (866) 632-9992. Submit your completed form or letter to USDA by: (1) mail: U.S. Department of Agriculture, Office of the Assistant Secretary for Civil Rights, 1400 Independence Avenue, SW, Washington, D.C. 20250-9410; (2) fax: (202) 690-7442; or (3) email: program.intake@usda.gov.

USDA is an equal opportunity provider, employer, and lender. 
Pregnancy and Lactation Subcommittee:

- Sharon Donovan, PhD, RD, University of Illinois, Urbana-Champaign, Subcommittee Chair

- Kathryn Dewey, PhD, University of California, Davis

- Rachel Novotny, PhD, RDN, LD, University of Hawaii

- Jamie Stang, PhD, MPH, RD, University of Minnesota

- Elsie Taveras, MD, MPH, Massachusetts General Hospital, Harvard Medical School, and Harvard T.H. Chan School of Public Health

- Ronald Kleinman, MD, Massachusetts General Hospital, Harvard Medical School, Vice-Chair of the 2020 Dietary Guidelines Advisory Committee

\section{Nutrition Evidence Systematic Review (NESR) Team:}

- Ramkripa Raghavan, DrPH, MPH, MSc, Analyst, Panum Groupi

- Julie Nevins, PhD, Analyst, Panum Groupi

- Sara Scinto-Madonich, MS, Analyst, Panum Groupi

- Gisela Butera, MLIS, MEd, Systematic Review Librarian, Panum Groupi

- Nancy Terry, MS, MLS, Biomedical Librarian, National Institutes of Health Library, U.S. Department of Health and Human Services (HHS)

- Julie Obbagy, PhD, RD, Project Lead, Office of Nutrition Guidance and Analysis (ONGA), Center for Nutrition Policy and Promotion (CNPP), Food and Nutrition Service (FNS), U.S. Department of Agriculture (USDA)

\section{Federal Liaisons:}

- Jean Altman, MS, ONGA, CNPP, FNS, USDA

- Meghan Adler, MS, RDN, ONGA, CNPP, USDA

- Jenna Fahle, MSPH, RDN, ONGA, CNPP, FNS, USDA (08/2019-11/2019)

\section{Project Leadership:}

- Eve Essery Stoody, PhD, Designated Federal Officer and Director, ONGA, CNPP, FNS, USDA

- Janet de Jesus, MS, RD, Nutrition Advisor, Office of Disease Prevention and Health Promotion, Office of the Assistant Secretary for Health, HHS

USDA and HHS implemented a process to identify topics and scientific questions to be examined by the 2020 Dietary Guidelines Advisory Committee. The Committee conducted its review of evidence in subcommittees for discussion by the full Committee during its public meetings. The role of the Committee members involved establishing all aspects of the protocol, which presented the plan for how they would examine the scientific evidence, including the inclusion and exclusion criteria; reviewing all studies that met the criteria they set; deliberating on the body of evidence for each question; and writing and grading the conclusion statements to be included in the scientific report the 2020 Committee submitted to USDA and HHS. The NESR team with assistance from Federal Liaisons and Project Leadership, supported the Committee by facilitating, executing, and documenting the work necessary to ensure the reviews were completed in accordance with NESR methodology.

\footnotetext{
' Under contract with the Food and Nutrition Service, United States Department of Agriculture.
} 
More information about the 2020 Dietary Guidelines Advisory Committee, including the process used to identify topics and questions, can be found at www.DietaryGuidelines.gov. More information about NESR can be found at NESR.usda.gov.

The Committee and NESR staff thank USDA's Agricultural Research Service for coordinating the peer review of this systematic review, and the Federal scientist peer reviewers for their time and expertise.

FUNDING SOURCE: United States Department of Agriculture, Food and Nutrition Service, Center for Nutrition Policy and Promotion, Alexandria, VA 


\section{TABLE OF CONTENTS}

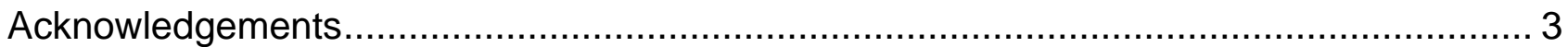

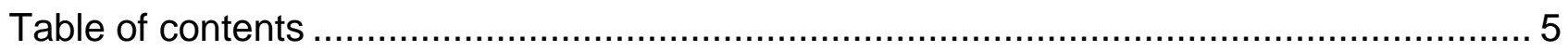

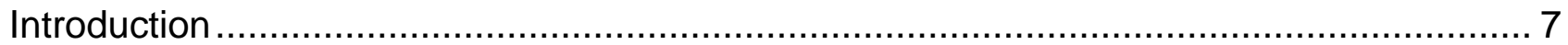

What is the relationship between maternal diet during pregnancy and lactation and risk of child food allergies and atopic allergic diseases? .......................................................... 9

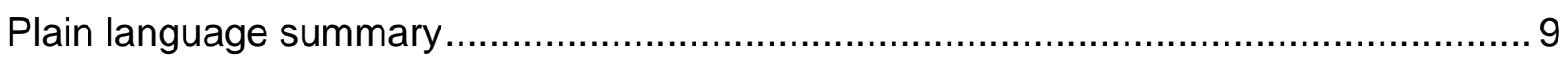

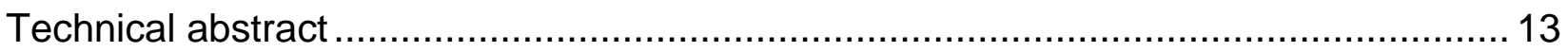

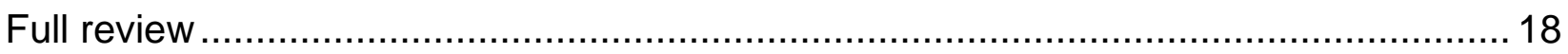

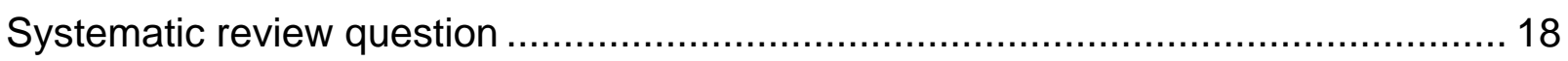

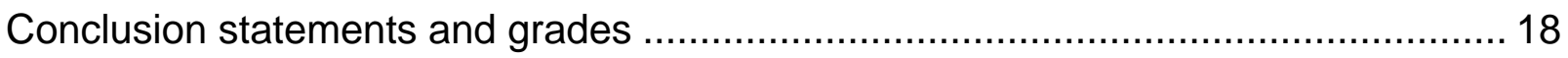

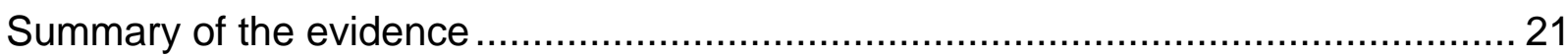

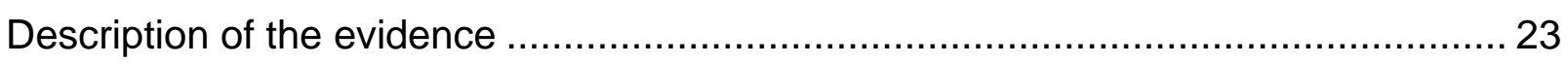

Evidence synthesis - Food allergy ............................................................... 31

Evidence synthesis - Atopic dermatitis/eczema ................................................. 34

Evidence synthesis - Allergic rhinitis ............................................................ 44

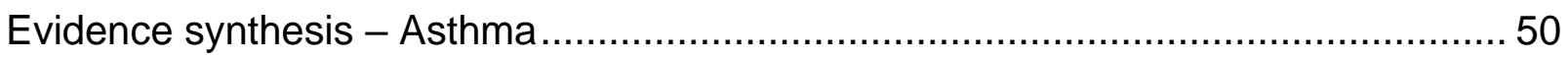

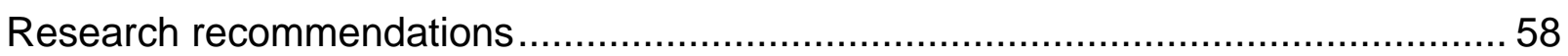

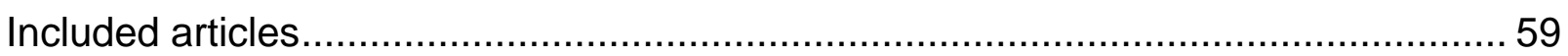

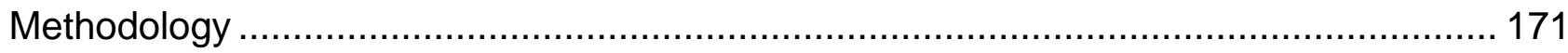

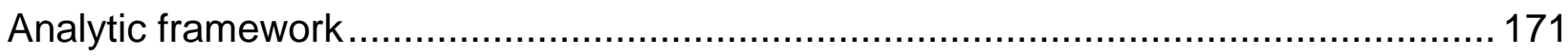

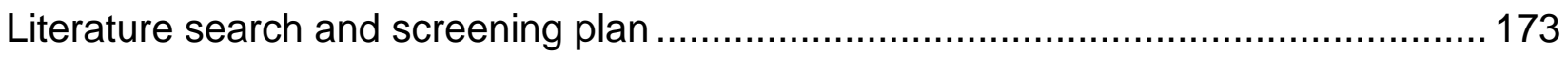

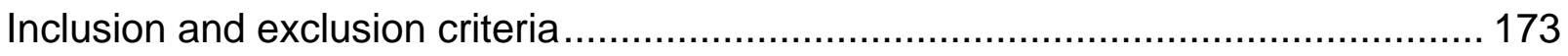

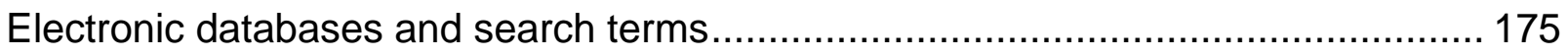

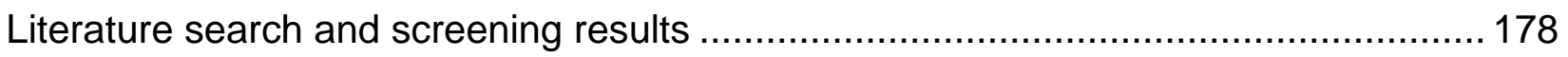

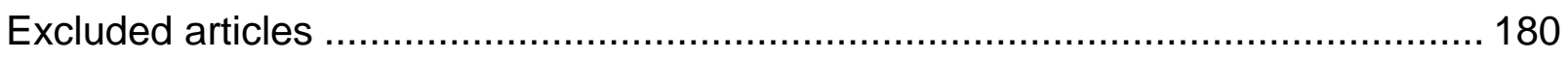

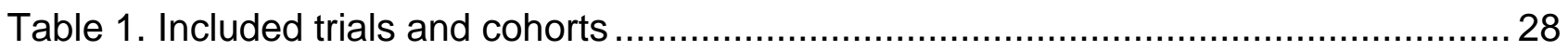

Table 2. Description of evidence on the relationship between maternal diet during

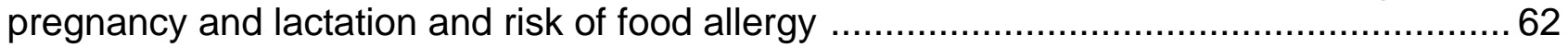

Table 3. Description of evidence on the relationship between maternal diet during

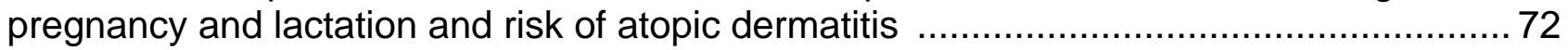

Table 4. Description of evidence on the relationship between maternal diet during pregnancy and lactation and risk of allergic rhinitis

Table 5. Description of evidence on the relationship between maternal diet during

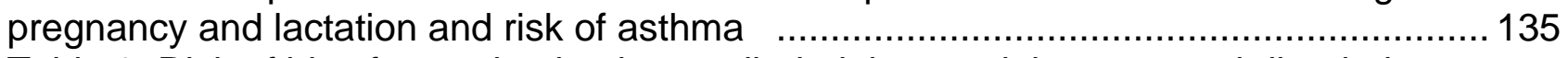

Table 6. Risk of bias for randomized controlled trials examining maternal diet during pregnancy and lactation and risk of child food allergies and atopic allergic diseases ..... 168 Table 7. Risk of bias for non-randomized controlled trials examining maternal diet during 
pregnancy and lactation and risk of child food allergies and atopic allergic diseases...... 168 Table 8. Risk of bias for observational studies examining maternal diet during pregnancy and lactation and risk of child food allergies and atopic allergic diseases 169

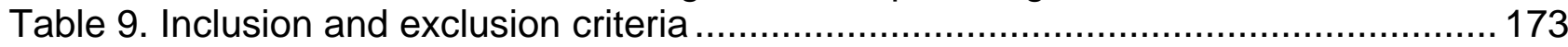

Table 10. Articles excluded after full text screening with rationale for exclusion............... 180

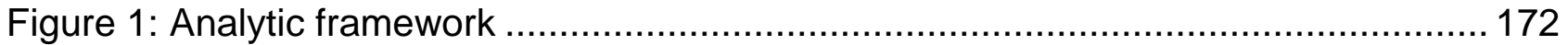

Figure 2: Flow chart of literature search and screening results................................. 179 
This document describes a systematic review conducted to answer the following question: What is the relationship between maternal diet during pregnancy and lactation and risk of child food allergies and atopic allergic diseases? This systematic review was conducted by the 2020 Dietary Guidelines Advisory Committee, supported by USDA's Nutrition Evidence Systematic Review (NESR).

More information about the 2020 Dietary Guidelines Advisory Committee is available at the following website: www.DietaryGuidelines.gov.

NESR specializes in conducting food- and nutrition-related systematic reviews using a rigorous, protocol-driven methodology. More information about NESR is available at the following website: NESR.usda.gov.

NESR's systematic review methodology involves developing a protocol, searching for and selecting studies, extracting data from and assessing the risk of bias of each included study, synthesizing the evidence, developing conclusion statements, grading the evidence underlying the conclusion statements, and recommending future research. A detailed description of the systematic reviews conducted for the 2020 Dietary Guidelines Advisory Committee, including information about methodology, is available on the NESR website: https://nesr.usda.gov/2020-dietary-guidelines-advisory-committee-systematic-reviews. In addition, starting on page 171, this document describes the final protocol as it was applied in the systematic review. A description of and rationale for modifications made to the protocol are described in the 2020 Dietary Guidelines Advisory Committee Report, Part D: Chapter 2. Food, Beverage, and Nutrient Consumption During Pregnancy and Chapter 3. Food, Beverage, and Nutrient Consumption During Lactation. 


\section{List of abbreviations}

\begin{tabular}{ll}
\hline Abbreviation & Full name \\
\hline ALSPAC & Avon Longitudinal Study of Parents and Children \\
\hline DP & Dietary pattern \\
\hline DII & Dietary Inflammation Index \\
\hline FFQ & Food frequency questionnaire \\
\hline HEI & Healthy Eating Index \\
\hline HDI & Human Development Index \\
\hline HHS & Department of Health and Human Services \\
\hline IgE & Immunoglobulin E \\
\hline NESR & Nutrition Evidence Systematic Review \\
\hline NRCT & Non-randomized controlled trial \\
\hline RCT & Randomized controlled trial \\
\hline SES & Socioeconomic status \\
\hline USDA & United States Department of Agriculture \\
\hline
\end{tabular}




\section{WHAT IS THE RELATIONSHIP BETWEEN MATERNAL DIET DURING PREGNANCY AND LACTATION AND RISK OF CHILD FOOD ALLERGIES AND ATOPIC ALLERGIC DISEASES?}

\section{PLAIN LANGUAGE SUMMARY}

\section{What is the question?}

- The question is: What is the relationship between maternal diet during pregnancy and lactation and risk of child food allergies and atopic allergic diseases?

What is the answer to the question?

\section{Diet during pregnancy and risk of child food allergy}

- Insufficient evidence is available to determine the relationship between lower or restricted consumption of cow milk products during pregnancy only, or during both pregnancy and lactation, and risk of food allergy in the child.

- Insufficient evidence is available to determine the relationship between peanuts, eggs, or wheat consumed during pregnancy and risk of food allergy in the child.

- Limited evidence suggests no relationship between soybean consumed during pregnancy and risk of food allergy in the child.

- No evidence is available to determine the relationship between maternal dietary patterns or fish, tree nuts and seeds, and foods not commonly considered to be allergens, such as meat, vegetables, and fruits consumed during pregnancy and risk of food allergy in the child.

\section{Diet during lactation and risk of child food allergy}

- Insufficient evidence is available to determine the relationship between lower or restricted consumption of cow milk products during both pregnancy and lactation and risk of food allergy in the child.

- No evidence is available to determine the relationship between maternal dietary patterns or cow milk products, eggs, peanuts, soybean, wheat, fish, tree nuts and seeds, and foods not commonly considered to be allergens, such as meat, vegetables, and fruits consumed during lactation and risk of food allergy in the child.

\section{Diet during pregnancy and risk of child atopic dermatitis/eczema}

- Moderate evidence indicates that lower or restricted consumption of cow milk products during pregnancy does not reduce the risk of atopic dermatitis/eczema in the child.

- Moderate evidence indicates that lower or restricted consumption of egg during pregnancy does not reduce the risk of atopic dermatitis/eczema in the child.

- Insufficient evidence is available to determine the relationship between cow milk products and eggs restricted during both pregnancy and lactation and risk of atopic dermatitis/eczema in the child.

- Limited evidence suggests that fish consumed during pregnancy does not increase the risk of atopic dermatitis/eczema in the child. 
- Limited evidence suggests that dietary patterns during pregnancy are not associated with risk of atopic dermatitis/eczema in the child.

- Insufficient evidence is available to determine the relationship between peanuts, soybean, wheat/cereal, yogurt and probiotic milk products, and foods not commonly considered to be allergens, such as meat, vegetables, and fruits, consumed during pregnancy and risk of atopic dermatitis/eczema in the child.

- No evidence is available to determine the relationship between tree nuts and seeds consumed during pregnancy and risk of atopic dermatitis/eczema in the child.

\section{Diet during lactation and risk of child atopic dermatitis/eczema}

- Insufficient evidence is available to determine the relationship between cow milk products restricted during both pregnancy and lactation, or during lactation only, and risk of atopic dermatitis/eczema in the child.

- Insufficient evidence is available to determine the relationship between egg consumption restricted during both pregnancy and lactation and risk of atopic dermatitis/eczema in the child.

- No evidence is available to determine the relationship between maternal dietary patterns or yogurt and probiotic milk products, eggs, fish, peanuts, tree nuts and seeds, soybean, wheat/cereal, and foods not commonly considered to be allergens, such as meat, vegetables, and fruits, consumed during lactation and risk of atopic dermatitis/eczema in the child.

\section{Diet during pregnancy and risk of child allergic rhinitis}

- Insufficient evidence is available to determine the relationship between cow milk products (fermented or non-fermented) consumed during pregnancy only, or during both pregnancy and lactation, and risk of allergic rhinitis in the child.

- Moderate evidence indicates that lower or restricted consumption of eggs during pregnancy does not reduce the risk of allergic rhinitis in the child.

- Limited evidence suggests that dietary patterns during pregnancy are not associated with risk of allergic rhinitis in the child.

- Insufficient evidence is available to determine the relationship between fish, peanuts, tree nuts, soybean, wheat, and foods not commonly considered to be allergens, such as meat, vegetables, and fruits consumed during pregnancy and risk of allergic rhinitis in the child.

- No evidence is available to determine the relationship between seeds consumed during pregnancy and the risk of allergic rhinitis in the child.

\section{Diet during lactation and risk of child allergic rhinitis}

- Insufficient evidence is available to determine the relationship between cow milk products consumed during both pregnancy and lactation, and risk of allergic rhinitis in the child.

- No evidence is available to determine the relationship between maternal dietary patterns or cow milk products, eggs, fish, peanuts, tree nuts and seeds, soybean, wheat, and foods not commonly considered to be allergens, such as meat, vegetables, and fruits consumed during lactation and risk of allergic rhinitis in the child. 


\section{Diet during pregnancy and risk of child asthma}

- Limited evidence suggests that a lower consumption of cow milk products during pregnancy does not reduce risk of asthma in the child.

- Insufficient evidence is available to determine the relationship between cow milk products consumed during both pregnancy and lactation and risk of asthma in the child.

- Limited evidence suggests no relationship between eggs consumed during pregnancy and risk of asthma in the child.

- Limited evidence suggests no relationship between fish consumed during pregnancy and risk of asthma in the child.

- Insufficient evidence is available to determine the relationship between maternal dietary patterns or peanuts, tree nuts, soybean, and other foods such as wheat/whole grains, vegetables, fruits, beverages, and margarine consumed during pregnancy and risk of asthma in the child.

- No evidence is available to determine the relationship between seeds consumed during pregnancy and risk of asthma in the child.

\section{Diet during lactation and risk of child asthma}

- Insufficient evidence is available to determine the relationship between cow milk products consumed during both pregnancy and lactation, or during lactation only, and risk of asthma in the child.

- Insufficient evidence is available to determine the relationship between fish, and other foods, such as margarine, oil, butter and butter-spreads, meat, and meat products consumed during lactation and risk of asthma in the child.

- No evidence is available to determine the relationship between maternal dietary patterns or eggs, peanuts, wheat, tree nuts and seeds, and soybean consumed during lactation and risk of asthma in the child.

\section{Why was this question asked?}

- This important public health question was identified by the U.S. Departments of Agriculture (USDA) and Health and Human Services (HHS) to be examined by the 2020 Dietary Guidelines Advisory Committee.

\section{How was this question answered?}

- The 2020 Dietary Guidelines Advisory Committee, Pregnancy and Lactation Subcommittee conducted a systematic review to answer this question with support from the Nutrition Evidence Systematic Review (NESR) team.

\section{What is the population of interest?}

- For the intervention/exposure, generally healthy women during pregnancy and/or lactation.

- For the outcome, infants and toddlers (birth to 24 months) and children and adolescents (ages 2-18 years).

\section{What evidence was found?}

\section{Pregnancyii}

- This systematic review included 36 articles that presented evidence from 15 studies that included only pregnant women and four studies that included both 
pregnant and lactating women.

- Studies looked at cow milk products, eggs, fish, soybean, wheat, peanuts, and tree nuts eaten during pregnancy alone, or during both pregnancy and lactation, as well as dietary patterns and foods not considered to be major allergens, such as meat, fruits, and vegetables eaten during pregnancy and/or lactation, with respect to the outcomes.

- There were a number of limitations including:

- Outcomes measured by methods with limited reliability.

- Only a few studies were well-designed randomized controlled trials. Studies were mostly observational and therefore, cause-effect relationships between diet during pregnancy and the child's risk of food allergy, atopic dermatitis, allergic rhinitis, and asthma, were difficult to determine.

- Potential bias of the studies.

- There was little racial/ethnic, socioeconomic, and age diversity in the studies.

\section{Lactation $^{\text {ii }}$}

- This systematic review included eight articles that presented evidence from four studies that included both pregnant and lactating women, and 2 studies that included only lactating women.

- Studies looked at cow milk products, eggs, fish, soybean, wheat, peanuts, tree nuts, as well as dietary patterns and foods not considered to be major allergens, such as meat, fruits, and vegetables eaten during both pregnancy and lactation, or lactation alone, with respect to the outcomes.

- There were a number of limitations including:

- Very few studies examined the relationship between what mothers ate during lactation alone and the child's risk of food allergy, atopic dermatitis, allergic rhinitis and asthma.

- Potential bias of the studies.

- There was little racial/ethnic, socioeconomic, and age diversity in the studies.

\section{How up-to-date is this systematic review?}

- This review searched for studies from January 1980 to January 2020.

\footnotetext{
ii Studies with interventions during both pregnancy and lactation are discussed in both pregnancy and lactation sections
} 


\section{TECHNICAL ABSTRACT}

\section{Background}

- This important public health question was identified by the U.S. Departments of Agriculture (USDA) and Health and Human Services (HHS) to be examined by the 2020 Dietary Guidelines Advisory Committee.

- The 2020 Dietary Guidelines Advisory Committee, Pregnancy and Lactation Subcommittee conducted a systematic review to answer this question with support from the Nutrition Evidence Systematic Review (NESR) team.

- The goal of this systematic review was to examine the following question: What is the relationship between maternal diet during pregnancy and lactation and risk of child food allergies and atopic allergic diseases?

\section{Conclusion statements and grades}

Food allergy

\section{Pregnancy}

- Insufficient evidence is available to determine the relationship between lower or restricted consumption of cow milk products during pregnancy only, or during both pregnancy and lactation, and risk of food allergy in the child. (Grade: Grade not assignable)

- Insufficient evidence is available to determine the relationship between peanuts, eggs, or wheat consumed during pregnancy and risk of food allergy in the child. (Grade: Grade not assignable)

- Limited evidence suggests no relationship between soybean consumed during pregnancy and risk of food allergy in the child. (Grade: Limited)

- No evidence is available to determine the relationship between maternal dietary patterns or fish, tree nuts and seeds, and foods not commonly considered to be allergens, such as meat, vegetables, and fruits consumed during pregnancy and risk of food allergy in the child. (Grade: Grade not assignable)

\section{Lactation}

- Insufficient evidence is available to determine the relationship between lower or restricted consumption of cow milk products during both pregnancy and lactation and risk of food allergy in the child. (Grade: Grade not assignable)

- No evidence is available to determine the relationship between maternal dietary patterns or cow milk products, eggs, peanuts, soybean, wheat, fish, tree nuts and seeds, and foods not commonly considered to be allergens, such as meat, vegetables, and fruits consumed during lactation and risk of food allergy in the child. (Grade: Grade not assignable)

\section{Atopic dermatitis/eczema}

\section{Pregnancy}

- Moderate evidence indicates that lower or restricted consumption of cow milk products during pregnancy does not reduce the risk of atopic dermatitis/eczema in the child. (Grade: Moderate)

- Moderate evidence indicates that lower or restricted consumption of egg during pregnancy does not reduce the risk of atopic dermatitis/eczema in the child. (Grade: 
Moderate)

- Insufficient evidence is available to determine the relationship between cow milk products and eggs restricted during both pregnancy and lactation and risk of atopic dermatitis/eczema in the child. (Grade: Grade not assignable)

- Limited evidence suggests that fish consumed during pregnancy does not increase the risk of atopic dermatitis/eczema in the child. (Grade: Limited)

- Limited evidence suggests that dietary patterns during pregnancy are not associated with risk of atopic dermatitis/eczema in the child. (Grade: Limited)

- Insufficient evidence is available to determine the relationship between peanuts, soybean, wheat/cereal, yogurt and probiotic milk products, and foods not commonly considered to be allergens, such as meat, vegetables, and fruits, consumed during pregnancy and risk of atopic dermatitis/eczema in the child. (Grade: Grade not assignable)

- No evidence is available to determine the relationship between tree nuts and seeds consumed during pregnancy and risk of atopic dermatitis/eczema in the child. (Grade: Grade not assignable)

\section{Lactation}

- Insufficient evidence is available to determine the relationship between cow milk products restricted during both pregnancy and lactation, or during lactation only, and risk of atopic dermatitis/eczema in the child. (Grade: Grade not assignable)

- Insufficient evidence is available to determine the relationship between egg consumption restricted during both pregnancy and lactation and risk of atopic dermatitis/eczema in the child. (Grade: Grade not assignable)

- No evidence is available to determine the relationship between maternal dietary patterns or yogurt and probiotic milk products, eggs, fish, peanuts, tree nuts and seeds, soybean, wheat/cereal, and foods not commonly considered to be allergens, such as meat, vegetables, and fruits, consumed during lactation and risk of atopic dermatitis/eczema in the child. (Grade: Grade not assignable)

\section{Allergic rhinitis}

\section{Pregnancy}

- Insufficient evidence is available to determine the relationship between cow milk products (fermented or non-fermented) consumed during pregnancy only, or during both pregnancy and lactation, and risk of allergic rhinitis in the child. (Grade: Grade not assignable)

- Moderate evidence indicates that lower or restricted consumption of eggs during pregnancy does not reduce the risk of allergic rhinitis in the child. (Grade: Moderate)

- Limited evidence suggests that dietary patterns during pregnancy are not associated with risk of allergic rhinitis in the child. (Grade: Limited)

- Insufficient evidence is available to determine the relationship between fish, peanuts, tree nuts, soybean, wheat, and foods not commonly considered to be allergens, such as meat, vegetables, and fruits consumed during pregnancy and risk of allergic rhinitis in the child. (Grade: Grade not assignable)

- No evidence is available to determine the relationship between seeds consumed during pregnancy and the risk of allergic rhinitis in the child. (Grade: Grade not 
assignable)

\section{Lactation}

- Insufficient evidence is available to determine the relationship between cow milk products consumed during both pregnancy and lactation, and risk of allergic rhinitis in the child. (Grade: Grade not assignable)

- No evidence is available to determine the relationship between maternal dietary patterns or cow milk products, eggs, fish, peanuts, tree nuts and seeds, soybean, wheat, and foods not commonly considered to be allergens, such as meat, vegetables, and fruits consumed during lactation and risk of allergic rhinitis in the child. (Grade: Grade not assignable)

\section{Asthma}

\section{Pregnancy}

- Limited evidence suggests that a lower consumption of cow milk products during pregnancy does not reduce risk of asthma in the child. (Grade: Limited)

- Insufficient evidence is available to determine the relationship between cow milk products consumed during both pregnancy and lactation and risk of asthma in the child. (Grade: Grade not assignable)

- Limited evidence suggests no relationship between eggs consumed during pregnancy and risk of asthma in the child. (Grade: Limited)

- Limited evidence suggests no relationship between fish consumed during pregnancy and risk of asthma in the child. (Grade: Limited)

- Insufficient evidence is available to determine the relationship between maternal dietary patterns or peanuts, tree nuts, soybean, and other foods such as wheat/whole grains, vegetables, fruits, beverages, and margarine consumed during pregnancy and risk of asthma in the child. (Grade: Grade not assignable)

- No evidence is available to determine the relationship between seeds consumed during pregnancy and risk of asthma in the child. (Grade: Grade not assignable)

\section{Lactation}

- Insufficient evidence is available to determine the relationship between cow milk products consumed during both pregnancy and lactation, or during lactation only, and risk of asthma in the child. (Grade: Grade not assignable)

- Insufficient evidence is available to determine the relationship between fish, and other foods, such as margarine, oil, butter and butter-spreads, meat, and meat products consumed during lactation and risk of asthma in the child. (Grade: Grade not assignable)

- No evidence is available to determine the relationship between maternal dietary patterns or eggs, peanuts, wheat, tree nuts and seeds, and soybean consumed during lactation and risk of asthma in the child. (Grade: Grade not assignable)

\section{Methods}

- A literature search was conducted using four databases (PubMed, Cochrane, Embase, and CINAHL) to identify articles that evaluated the intervention or exposure of maternal diet during pregnancy and lactation and the outcomes of child food allergies and atopic allergic diseases. A manual search was conducted to 
identify articles that may not have been included in the electronic databases searched. Articles were screened by two NESR analysts independently for inclusion based on pre-determined criteria.

- Data extraction and risk of bias assessment were conducted for each included study, and both were checked for accuracy. The Committee qualitatively synthesized the body of evidence to inform development of a conclusion statement(s), and graded the strength of evidence using pre-established criteria for risk of bias, consistency, directness, precision, and generalizability.

\section{Summary of the evidence}

\section{Pregnancyiii}

- This systematic review included 36 articles from five randomized controlled trials (RCTs), one non-RCT (NRCT), and 13 prospective cohort studies that assessed the association between maternal diet and risk of food allergy, atopic dermatitis/eczema, allergic rhinitis, and asthma in the child occurring from birth through $18 \mathrm{y}$ of age. The articles were published between 1987 and 2020 and consisted of:

- Thirty articles from 15 studies that included only pregnant women.

- Six articles from four studies that included both pregnant and lactating women.

- Six articles from two RCTs and two prospective cohort studies examined maternal avoidance and/or consumption of cow milk products, eggs, soybean, wheat, and peanuts during pregnancy alone, or during both pregnancy and lactation, in relation to risk of food allergy in the child from birth through $18 \mathrm{y}$ of age.

- Twenty-five articles from five RCTs, one NRCT, and 10 prospective cohort studies examined maternal dietary patterns and consumption and/or avoidance of cow milk products, eggs, fish, soybean, wheat, peanuts, tree nuts, and other foods not commonly considered to be allergens during pregnancy alone, or during both pregnancy and lactation, in relation to risk of atopic dermatitis/eczema in the child from birth through age $18 \mathrm{y}$.

- Sixteen articles from four RCTs and six prospective cohort studies examined maternal dietary patterns and avoidance and/or consumption of cow milk products, eggs, fish, soybean, wheat, peanuts, tree nuts, and other foods not commonly considered to be allergens during pregnancy alone, or during both pregnancy and lactation, in relation to risk of allergic rhinitis in the child from birth through $18 \mathrm{y}$ of age.

- Nineteen articles from two RCTs and eight prospective cohort studies examined maternal dietary patterns and avoidance and/or consumption of cow milk products, eggs, fish, soybean, peanuts, tree nuts, and other foods during pregnancy alone, or during both pregnancy and lactation, in relation to risk of asthma in the child from 2 through $18 \mathrm{y}$ of age.

- No articles were identified that examined maternal consumption of seeds during pregnancy in relation to risk of atopic outcomes in the child from birth through $18 \mathrm{y}$ of age.

- The ability to draw strong conclusions was limited by the following issues:

- Few RCTs have been conducted and thus, data were primarily observational in nature, limiting the ability to determine causal effects of consumption or avoidance of different foods during pregnancy and risk of 
atopic dermatitis, food allergies, allergic rhinitis, and asthma in the child.

- Key confounders were not consistently controlled for in most of the studies.

- Studies had risk-of-bias issues, such as self-reported outcomes and selection bias.

- People with lower SES, adolescents, and racially and ethnically diverse populations were underrepresented in the body of evidence.

\section{Lactation $^{\text {iii }}$}

- This systematic review included eight articles from four randomized controlled trials (RCTs), one non-RCT (NRCT), and one prospective cohort study that assessed the relationship between maternal diet during both pregnancy and lactation, or during lactation alone, and risk of food allergy, atopic dermatitis/eczema, allergic rhinitis, and asthma in the child occurring from birth through $18 \mathrm{y}$ of age. The included articles were published between 1989 and 2013.

- Six articles from four studies included both pregnant and lactating women.

- Two studies included only lactating women.

- Four articles from two RCTs examined maternal avoidance of cow milk products, eggs, soybean, wheat, and peanuts during both pregnancy and lactation, in relation to risk of food allergy and allergic rhinitis in the child from birth through $18 \mathrm{y}$ of age. None of these studies were conducted exclusively in lactating women.

- Seven articles from four RCTs and one NRCT examined maternal avoidance of cow milk products, eggs, soybean, wheat, and peanuts during both pregnancy and lactation, or during lactation alone, in relation to the risk of atopic dermatitis/eczema in the child from birth through $18 \mathrm{y}$ of age. Of these, only one RCT was conducted exclusively in lactating women.

- Four articles from two RCTs and one prospective cohort study examined maternal avoidance and/or consumption of cow milk products, eggs, fish, soybean, peanuts, wheat, and other foods during both pregnancy and lactation, or during lactation alone, in relation to risk of asthma in the child from 2 through $18 \mathrm{y}$ of age. Of these, one prospective cohort study was conducted exclusively in lactating women.

- No articles were identified that examined maternal avoidance or consumption of seeds during lactation in relation to the risk of atopic outcomes in the child from birth through $18 \mathrm{y}$ of age.

- The ability to draw strong conclusions was limited by the following issues:

- Very few studies assessed the relationship between maternal diet during lactation alone and risk of atopic dermatitis, food allergy, allergic rhinitis, and asthma.

- Key confounders were not consistently controlled for in most of the studies.

- Women with lower socioeconomic status (SES), adolescents, and racially and ethnically diverse populations were underrepresented in the body of evidence.

iii Studies with interventions during both pregnancy and lactation are discussed in both pregnancy and lactation sections 


\section{FULL REVIEW}

\section{Systematic review question}

What is the relationship between maternal diet during pregnancy and lactation and risk of child food allergies and atopic allergic diseases?

\section{Conclusion statements and grades}

\section{Food allergy}

\section{Pregnancy}

Insufficient evidence is available to determine the relationship between lower or restricted consumption of cow milk products during pregnancy only, or during both pregnancy and lactation, and risk of food allergy in the child. (Grade: Grade not assignable)

Insufficient evidence is available to determine the relationship between peanuts, eggs, or wheat consumed during pregnancy and risk of food allergy in the child. (Grade: Grade not assignable)

Limited evidence suggests no relationship between soybean consumed during pregnancy and risk of food allergy in the child. (Grade: Limited)

No evidence is available to determine the relationship between maternal dietary patterns or fish, tree nuts and seeds, and foods not commonly considered to be allergens, such as meat, vegetables, and fruits consumed during pregnancy and risk of food allergy in the child. (Grade: Grade not assignable)

\section{Lactation}

Insufficient evidence is available to determine the relationship between lower or restricted consumption of cow milk products during both pregnancy and lactation and risk of food allergy in the child. (Grade: Grade not assignable)

No evidence is available to determine the relationship between maternal dietary patterns or cow milk products, eggs, peanuts, soybean, wheat, fish, tree nuts and seeds, and foods not commonly considered to be allergens, such as meat, vegetables, and fruits consumed during lactation and risk of food allergy in the child. (Grade: Grade not assignable)

\section{Atopic dermatitis/eczema}

\section{Pregnancy}

Moderate evidence indicates that lower or restricted consumption of cow milk products during pregnancy does not reduce the risk of atopic dermatitis/eczema in the child. (Grade: Moderate)

Moderate evidence indicates that lower or restricted consumption of egg during pregnancy does not reduce the risk of atopic dermatitis/eczema in the child. (Grade: Moderate)

Insufficient evidence is available to determine the relationship between cow milk products and eggs restricted during both pregnancy and lactation and risk of atopic dermatitis/eczema in the child. (Grade: Grade not assignable) 
Limited evidence suggests that fish consumed during pregnancy does not increase the risk of atopic dermatitis/eczema in the child. (Grade: Limited)

Limited evidence suggests that dietary patterns during pregnancy are not associated with risk of atopic dermatitis/eczema in the child. (Grade: Limited)

Insufficient evidence is available to determine the relationship between peanuts, soybean, wheat/cereal, yogurt and probiotic milk products, and foods not commonly considered to be allergens, such as meat, vegetables, and fruits, consumed during pregnancy and risk of atopic dermatitis/eczema in the child. (Grade: Grade not assignable)

No evidence is available to determine the relationship between tree nuts and seeds consumed during pregnancy and risk of atopic dermatitis/eczema in the child. (Grade: Grade not assignable)

\section{Lactation}

Insufficient evidence is available to determine the relationship between cow milk products restricted during both pregnancy and lactation, or during lactation only, and risk of atopic dermatitis/eczema in the child. (Grade: Grade not assignable)

Insufficient evidence is available to determine the relationship between egg consumption restricted during both pregnancy and lactation and risk of atopic dermatitis/eczema in the child. (Grade: Grade not assignable)

No evidence is available to determine the relationship between maternal dietary patterns or yogurt and probiotic milk products, eggs, fish, peanuts, tree nuts and seeds, soybean, wheat/cereal, and foods not commonly considered to be allergens, such as meat, vegetables, and fruits, consumed during lactation and risk of atopic dermatitis/eczema in the child. (Grade: Grade not assignable)

\section{Allergic rhinitis}

\section{Pregnancy}

Insufficient evidence is available to determine the relationship between cow milk products (fermented or non-fermented) consumed during pregnancy only, or during both pregnancy and lactation, and risk of allergic rhinitis in the child. (Grade: Grade not assignable)

Moderate evidence indicates that lower or restricted consumption of eggs during pregnancy does not reduce the risk of allergic rhinitis in the child. (Grade: Moderate)

Limited evidence suggests that dietary patterns during pregnancy are not associated with risk of allergic rhinitis in the child. (Grade: Limited)

Insufficient evidence is available to determine the relationship between fish, peanuts, tree nuts, soybean, wheat, and foods not commonly considered to be allergens, such as meat, vegetables, and fruits consumed during pregnancy and risk of allergic rhinitis in the child. (Grade: Grade not assignable)

No evidence is available to determine the relationship between seeds consumed during pregnancy and the risk of allergic rhinitis in the child. (Grade: Grade not assignable) 


\section{Lactation}

Insufficient evidence is available to determine the relationship between cow milk products consumed during both pregnancy and lactation, and risk of allergic rhinitis in the child. (Grade: Grade not assignable)

No evidence is available to determine the relationship between maternal dietary patterns or cow milk products, eggs, fish, peanuts, tree nuts and seeds, soybean, wheat, and foods not commonly considered to be allergens, such as meat, vegetables, and fruits consumed during lactation and risk of allergic rhinitis in the child. (Grade: Grade not assignable)

\section{Asthma}

\section{Pregnancy}

Limited evidence suggests that a lower consumption of cow milk products during pregnancy does not reduce risk of asthma in the child. (Grade: Limited)

Insufficient evidence is available to determine the relationship between cow milk products consumed during both pregnancy and lactation and risk of asthma in the child. (Grade: Grade not assignable)

Limited evidence suggests no relationship between eggs consumed during pregnancy and risk of asthma in the child. (Grade: Limited)

Limited evidence suggests no relationship between fish consumed during pregnancy and risk of asthma in the child. (Grade: Limited)

Insufficient evidence is available to determine the relationship between maternal dietary patterns or peanuts, tree nuts, soybean, and other foods such as wheat/whole grains, vegetables, fruits, beverages, and margarine consumed during pregnancy and risk of asthma in the child. (Grade: Grade not assignable)

No evidence is available to determine the relationship between seeds consumed during pregnancy and risk of asthma in the child. (Grade: Grade not assignable)

\section{Lactation}

Insufficient evidence is available to determine the relationship between cow milk products consumed during both pregnancy and lactation, or during lactation only, and risk of asthma in the child. (Grade: Grade not assignable)

Insufficient evidence is available to determine the relationship between fish, and other foods, such as margarine, oil, butter and butter-spreads, meat, and meat products consumed during lactation and risk of asthma in the child. (Grade: Grade not assignable)

No evidence is available to determine the relationship between maternal dietary patterns or eggs, peanuts, wheat, tree nuts and seeds, and soybean consumed during lactation and risk of asthma in the child. (Grade: Grade not assignable) 


\section{Summary of the evidence}

\section{Pregnancyiv}

- This systematic review included 36 articles from five randomized controlled trials (RCTs), one non-RCT (NRCT), and 13 prospective cohort studies that assessed the association between maternal diet and risk of food allergy, atopic dermatitis/eczema, allergic rhinitis, and asthma in the child occurring from birth through 18 years of age. The articles were published between 1987 and 2020 and consisted of:

- Thirty articles from 15 studies that included only pregnant women.

- Six articles from four studies that included both pregnant and lactating women.

- Six articles from two RCTs and two prospective cohort studies examined maternal avoidance and/or consumption of cow milk products, eggs, soybean, wheat, and peanuts during pregnancy alone, or during both pregnancy and lactation, in relation to risk of food allergy in the child from birth through $18 \mathrm{y}$ of age.

- Twenty-five articles from five RCTs, one NRCT, and 10 prospective cohort studies examined maternal dietary patterns and consumption and/or avoidance of cow milk products, eggs, fish, soybean, wheat, peanuts, tree nuts, and other foods not commonly considered to be allergens during pregnancy alone, or during both pregnancy and lactation, in relation to risk of atopic dermatitis/eczema in the child from birth through age 18 years.

- Sixteen articles from four RCTs and six prospective cohort studies examined maternal dietary patterns and avoidance and/or consumption of cow milk products, eggs, fish, soybean, wheat, peanuts, tree nuts, and other foods not commonly considered to be allergens during pregnancy alone, or during both pregnancy and lactation, in relation to risk of allergic rhinitis in the child from birth through $18 \mathrm{y}$ of age.

- Nineteen articles from two RCTs and eight prospective cohort studies examined maternal dietary patterns and avoidance and/or consumption of cow milk products, eggs, fish, soybean, peanuts, tree nuts, and other foods during pregnancy alone, or during both pregnancy and lactation, in relation to risk of asthma in the child from 2 through $18 \mathrm{y}$ of age.

- No articles were identified that examined maternal consumption of seeds during pregnancy in relation to risk of atopic outcomes in the child from birth through $18 \mathrm{y}$ of age.

- The ability to draw strong conclusions was limited by the following issues:

- Few RCTs have been conducted and thus, data were primarily observational in nature, limiting the ability to determine causal effects of consumption or avoidance of different foods during pregnancy and risk of atopic dermatitis, food allergies, allergic rhinitis, and asthma in the child.

- Key confounders were not consistently controlled for in most of the studies.

- Studies had risk-of-bias issues, such as self-reported outcomes and selection bias.

- People with lower SES, adolescents, and racially and ethnically diverse populations were underrepresented in the body of evidence. 


\section{Lactation $^{\text {iv }}$}

- This systematic review included eight articles from four randomized controlled trials (RCTs), one non-RCT (NRCT), and one prospective cohort study that assessed the relationship between maternal diet during both pregnancy and lactation, or during lactation alone, and risk of food allergy, atopic dermatitis/eczema, allergic rhinitis, and asthma in the child occurring from birth through 18 years of age. The included articles were published between 1989 and 2013.

- Six articles from four studies included both pregnant and lactating women.

- Two studies included only lactating women.

- Four articles from two RCTs examined maternal avoidance of cow milk products, eggs, soybean, wheat, and peanuts during both pregnancy and lactation, in relation to risk of food allergy and allergic rhinitis in the child from birth through $18 \mathrm{y}$ of age. None of these studies were conducted exclusively in lactating women.

- Seven articles from four RCTs and one NRCT examined maternal avoidance of cow milk products, eggs, soybean, wheat, and peanuts during both pregnancy and lactation, or during lactation alone, in relation to the risk of atopic dermatitis/eczema in the child from birth through 18 years of age. Of these, only one RCT was conducted exclusively in lactating women.

- Four articles from two RCTs and one prospective cohort study examined maternal avoidance and/or consumption of cow milk products, eggs, fish, soybean, peanuts, wheat, and other foods during both pregnancy and lactation, or during lactation alone, in relation to risk of asthma in the child from 2 through $18 \mathrm{y}$ of age. Of these, one prospective cohort study was conducted exclusively in lactating women.

- No articles were identified that examined maternal avoidance or consumption of seeds during lactation in relation to the risk of atopic outcomes in the child from birth through $18 \mathrm{y}$ of age.

- The ability to draw strong conclusions was limited by the following issues:

- Very few studies assessed the relationship between maternal diet during lactation alone and risk of atopic dermatitis, food allergy, allergic rhinitis, and asthma.

- Key confounders were not consistently controlled for in most of the studies.

- Women with lower socioeconomic status (SES), adolescents, and racially and ethnically diverse populations were underrepresented in the body of evidence.

iv Studies with interventions during both pregnancy and lactation are discussed in both pregnancy and lactation sections 


\section{Description of the evidence}

This systematic review included articles that addressed the relationship between maternal diet during pregnancy and lactation and risk of child food allergies and atopic diseases. The search included articles from very high and high Human Development Index $(\mathrm{HDI})^{v}$ countries and the search timeframe spanned from January 1980 to January 2020. The following study designs were included: RCTs, NRCTs, prospective and retrospective cohort studies, and nested case-control studies.

The outcomes considered in this review were food allergies, atopic dermatitis, allergic rhinitis, and asthma in the child (birth to 18 years). Food allergy was defined as a diagnosis based on either the gold standard of a double-blind, placebo-controlled oral food challenge or as parental report of clinical history with blood immunoglobulin $\mathrm{E}$ ( $\operatorname{lgE}$ ) levels $\geq 0.35 \mathrm{kU} / \mathrm{L}$ and/or skin prick test wheal $\geq 3 \mathrm{~mm}$. Because of the difficulty in diagnosing asthma during infancy and toddlerhood, only those studies that assessed asthma in children who were at least 2 years or older were included in this systematic review.

In total, 38 articles were included in the body of evidence. The evidence in this review is presented based on the outcomes: 1) Food allergy; 2) Atopic dermatitis/eczema; 3) Allergic rhinitis; and 4) Asthma.

\section{Food Allergy}

Six articles assessed the relationship between maternal diet and risk of food allergy in the child, including one from a Japanese RCT, ${ }^{8}$ three from a U.S.-based RCT, ${ }^{34-36}$ and one each from two prospective cohort studies. ${ }^{4,12} \mathrm{~A}$ total of four distinct groups of trials/cohort studies were represented in this body of evidence. References are included for each study in Table 1 with trial/cohort names or locations when names were unavailable.

One RCT $\mathrm{T}^{34-36}$ and one prospective cohort study ${ }^{4}$ were conducted in the U.S. One study each was conducted in $\mathrm{Japan}^{8}$ and the U.K. ${ }^{12}$

\section{Participant characteristics}

- Sample size of the studies ranged from $350^{8}$ to $13,971 .^{12}$

- Among the studies that reported these characteristics, all included participants were adult women with singleton pregnancies.

- Health characteristics:

- RCTs recruited participants who exclusively $y^{34-36}$ or predominantly ${ }^{8}$ had a personal and/or family history of allergies.

- In the prospective cohort studies, ${ }^{4,12}$ participants represented the general

\footnotetext{
$\checkmark$ The Human Development classification was based on the Human Development Index (HDI) ranking from the year the study intervention occurred or data were collected (UN Development Program. HDI 1990-2017 HDRO calculations based on data from UNDESA (2017a), UNESCO Institute for Statistics (2018), United Nations Statistics Division (2018b), World Bank (2018b), Barro and Lee (2016) and IMF (2018). Available from: http://hdr.undp.org/en/data). If the study did not report the year in which the intervention occurred or data were collected, the HDI classification for the year of publication was applied. HDI values are available from 1980, and then from 1990 to present. If a study was conducted prior to 1990, the HDI classification from 1990 was applied. When a country was not included in the HDI ranking, the current country classification from the World Bank was used instead (The World Bank. World Bank country and lending groups. Available from: https://datahelpdesk.worldbank.org/knowledgebase/articles/906519-world- country-and-lendinggroups).
} 
population and the majority were not from families with high risk of allergies.

- Race/ethnicity:

○ The U.S.-based RCT ${ }^{34-36}$ and Project Viva cohort study ${ }^{4}$ noted that the participants were predominantly White (defined as $\geq 50$ percent of the participants). One RCT included participants that were 100 percent from Japan. ${ }^{8}$

- One prospective cohort study did not report race/ethnicity of participants. $^{12}$

- Socio-economic status:

- Maternal education:

- One U.S.-based RCT $\mathrm{RT}^{34-36}$ and prospective cohort study ${ }^{4}$ reported that the majority of participants had some higher education.

- One RCT ${ }^{8}$ and one cohort study ${ }^{12}$ did not report on maternal education.

- Income/Employment Status:

- The majority of women were employed and/or from middle-to-high income households in a U.S.-based trial ${ }^{34-36}$ and Project Viva cohort study 4 that reported household income and/or participant employment status.

- One $\mathrm{RCT}^{8}$ and one cohort study ${ }^{12}$ did not report on income or employment status.

\section{Atopic dermatitis/eczema}

Twenty six articles, including eight from six RCTs, ${ }^{7,8,14,16,34-37}$ one NRCT, ${ }^{10}$ and 17 from 10 prospective cohort studies ${ }^{1,3-5,11,13,15,16,21-29,31}$ assessed the association between maternal diet and risk of atopic dermatitis. A total of 18 distinct groups of trials/cohort studies were represented in this body of evidence. References are included for each study in Table 1 with trial/cohort names or locations when names were unavailable.

One $\mathrm{RCT}^{8}$ and two prospective cohort studies were conducted in Japan. ${ }^{21-26,28}$ In addition, three studies were conducted in the U.K., ${ }^{1,16,29,31}$ and two studies each in Sweden ${ }^{7,14}$ and the U.S., ${ }^{4,34-36}$ and one each in the Netherlands, ${ }^{13,27}$ Norway, ${ }^{3}$ Germany, ${ }^{10}$ Singapore, ${ }^{15}$ Thailand, ${ }^{37}$ Spain/Greece, ${ }^{5}$ and Poland/U.S. ${ }^{11}$

\section{Participant characteristics}

- Sample size of the studies ranged from $44^{16}$ to $40,641 .^{3}$

- From the studies that reported these characteristics, almost all included participants who were adult women with singleton pregnancies.

○ Bédard et al $^{1}$ reported 2.5 percent twin pregnancies in the ALSPAC cohort, Falth-Magnusson et $\mathrm{al}^{7}$ and Lovegrove et $\mathrm{al}^{16}$ each included one pair of twins in the trial, and Lilja et al ${ }^{14}$ included four pairs of twins.

- Health characteristics:

- The NRCT ${ }^{10}$ and all the RCTs recruited participants who exclusively $7,14,16,34-37$ or predominantly ${ }^{8}$ had a personal and/or family history of allergies. 
- In the prospective cohort studies, ${ }^{1,3-5,11,13,15,16,21-29,31}$ participants represented the general population and the majority were not from families with high risk of allergies.

- Race/ethnicity:

o The U.S.-based RCT ${ }^{34-36}$ and Project Viva cohort, ${ }^{4}$ as well as the U.K.based ALSPAC cohort, ${ }^{1,29}$ noted that the participants were predominantly White (defined as $\geq 50$ percent of the participants). The Growing Up in Singapore Towards Healthy Outcomes (GUSTO) cohort based in Singapore reported that all of its participants were of Asian race. ${ }^{15}$ One $\mathrm{RCT}^{8}$ and two prospective cohort studies ${ }^{21-26,28}$ included participants that were 100 percent from Japan, while one article from the Generation R cohort specified that participants were Dutch women. ${ }^{13}$

- Four RCTs, $7,14,16,37$ the NRCT, ${ }^{10}$ and four cohort articles did not report race/ethnicity of the mothers. . $^{3,5,11,27,31}$

- Socio-economic status:

- Maternal education:

- One U.S.-based RCT, ${ }^{34-36}$ the NRCT, ${ }^{10}$ and seven prospective cohort studies $^{1,4,11,13,15,21-27,29}$ reported that the majority of participants had some higher education. The study based in Greece and Spain reported that $\sim 34$ percent of participants had a high educational level, ${ }^{5}$ while participants in one U.K.-based cohort were a median age of 18.5 years when they left full-time education. ${ }^{31}$

- Five RCTs R $^{7,8,14,16,37}$ did not report maternal education.

- Income/Employment Status:

- The majority of women were employed and/or from middle-to-high income households in one U.S.-based $\mathrm{RCT}^{34-36}$ and four prospective cohort studies. ${ }^{4,13,21-28,31}$

- About 17 percent of participants in one ALSPAC cohort article ${ }^{1}$ responded yes to experiencing financial difficulties, while $\sim 26$ percent of participants in a second ALSPAC cohort article reported many financial difficulties. ${ }^{29}$

- Five RCTs, ${ }^{7,8,14,16,37}$ the NRCT, ${ }^{10}$ and four cohorts ${ }^{3,5,11,15}$ did not report income or employment status.

\section{Allergic rhinitis}

Sixteen articles, including six from four $\mathrm{RCTs}^{7,8,14,34-36}$ and 10 from six prospective cohort studies, ${ }^{1,3,4,15,17-20,29,31}$ assessed the association between maternal diet and risk of allergic rhinitis in the child. A total of 10 distinct groups of trials/cohort studies were represented in this body of evidence. References are included for each study in Table 1 with trial/cohort names or locations when names were unavailable. One $\mathrm{RCT}^{34-36}$ and one prospective cohort study ${ }^{4}$ were conducted in the U.S., while one prospective cohort study was conducted in Denmark. ${ }^{17-20}$ Two studies were conducted in Sweden, ${ }^{7,14}$ while two cohort studies were conducted in the U.K. ${ }^{1,29,31}$ One study each was conducted in Singapore, ${ }^{15}$ Norway, ${ }^{3}$ and Japan. ${ }^{8}$

\section{Participant characteristics}

- Sample size of the studies ranged from $171^{14}$ to $61,909 .{ }^{18}$ 
- From the studies that reported these characteristics, almost all included participants who were adult women with singleton pregnancies.

$\circ \sim 1$ percent of participants were younger than 20 years in the Danish National Birth Cohort. ${ }^{17-20}$

- Bédard et al $^{1}$ reported 2.5 percent twin pregnancies in the ALSPAC cohort, Falth-Magnusson et $\mathrm{al}^{7}$ included one pair of twins in the trial, and Lilja et $\mathrm{al}^{14}$ included four pairs of twins.

- Health characteristics:

- RCTs recruited participants who exclusively,14,34-36 or predominantly ${ }^{8}$ had a personal and/or family history of allergies.

- In the prospective cohort studies, , ,3,4,15,17-20,29,31 participants represented the general population and the majority were not from families with high risk of allergies.

- Race/ethnicity:

○ The U.S.-based RCT ${ }^{34-36}$ and Project Viva cohort study, ${ }^{4}$ as well as the U.K.-based ALSPAC cohort, ${ }^{1,29}$ noted that the participants were predominantly White (defined as $\geq 50$ percent of the participants). The GUSTO cohort based in Singapore reported that all of its participants were of Asian race. ${ }^{15}$ One RCT included participants that were 100 percent from Japan. ${ }^{8}$

- Two RCTs R $^{7,14}$ and three prospective cohort studies $3,17-20,31$ did not report race/ethnicity of participants.

- Socio-economic status:

- Maternal education:

- One U.S.-based RCT ${ }^{34-36}$ and four prospective cohort studies ${ }^{1,3,4,15,29}$ reported that the majority of participants had some higher education. Participants in one U.K.-based cohort were a median age of 18.5 years when they left full-time education. ${ }^{31}$

- Three RCTs ${ }^{7,8,14}$ and one Danish cohort did not report on maternal education. ${ }^{17-20}$

- Income/Employment Status:

- The majority of women were employed and/or from middle-to-high income households in one U.S.-based $\mathrm{RCT}^{34-36}$ and the Project Viva cohort, ${ }^{4}$ one U.K.-based cohort, ${ }^{31}$ and the Danish National Birth Cohort. ${ }^{17-20}$

- About 17 percent of participants in one ALSPAC cohort report ${ }^{1}$ responded yes to experiencing financial difficulties, while $\sim 26$ percent of participants in a second ALSPAC cohort report indicated many financial difficulties. ${ }^{29}$

- Three RCTs R $^{7,8,14}$ and two cohorts ${ }^{3,15}$ did not report on income or employment status.

\section{Asthma}

Twenty articles, including three from two RCTs ${ }^{8,34,36}$ and 17 from nine prospective cohort studies, ${ }^{1-4,6,9,17-20,27,29-33,38}$ assessed the association between maternal diet and risk of asthma in the child. A total of 11 distinct groups of trials/cohort studies were represented in this body of evidence. References are included for each study in Table 1 with trial/cohort names or locations when names were unavailable. 
One RCT ${ }^{34,36}$ and one prospective cohort study $y^{4,9,33}$ were conducted in the U.S. One prospective cohort study was conducted in Denmark, ${ }^{17-20}$ while two cohort studies were conducted in the U.K. ${ }^{1,2,29,31}$ One cohort study was conducted in Ireland, ${ }^{6,30}$ while two cohort studies were conducted in the Netherlands. ${ }^{27,32}$ One study each was conducted in Norway, ${ }^{3}$ Finland, ${ }^{38}$ and Japan. ${ }^{8}$

\section{Participant characteristics:}

- Sample size of the studies ranged from $350^{8}$ to $61,909 .{ }^{18}$

- Among the studies that reported these characteristics, majority of included participants were adult women with singleton pregnancies.

- About 1 percent of participants were younger than 20 years in the Danish National Birth Cohort, ${ }^{17-20}$ while 2 percent of participants were younger than 18 years in an Irish cohort. 6,30

- Bédard et al ${ }^{1,2}$ reported $\sim 2.5$ percent twin pregnancies in the ASLPAC cohort.

- Health characteristics:

- RCTs recruited participants who exclusively $y^{34,36}$ or predominantly ${ }^{8}$ had a personal and/or family history of allergies.

- In the prospective cohort studies, ${ }^{1-4,6,9,17-20,27,29-33,38}$ participants represented the general population and the majority were not from families with high risk of allergies.

- Race/ethnicity:

- The U.S.-based RCT ${ }^{34,36}$ and Project Viva cohort study, 4,9,33 as well as the U.K.-based ALSPAC cohort, ${ }^{1,2,29}$ noted that the participants were predominantly White (defined as $\geq 50$ percent of the participants). Participants in the Lifeways Cross-Generation cohort study were 100 percent Irish-born. ${ }^{6,30}$ One RCT included participants that were 100 percent from Japan. ${ }^{8}$

- Seven prospective cohort studies did not report race/ethnicity of participants. ${ }^{3,17-20,27,31,32,38}$

- Socio-economic status:

- Maternal education:

- One U.S.-based RCT ${ }^{34,36}$ and six prospective cohort studies ${ }^{1-}$ $4,6,9,27,29,30,33$ reported that the majority of participants had some higher education. Participants in one U.K.-based cohort were a median age of 18.5 years, when they left full-time education, ${ }^{31}$ while a cohort based in the Netherlands reported that $~ 34$ percent of participants had a high educational level at baseline. ${ }^{32}$

- One $\mathrm{RCT}^{8}$ and two cohort studies did not report maternal education level. ${ }^{17-20,38}$

- Income/Employment Status:

- The majority of women were employed and/or from middle-to-high income households in one U.S.-based RCT ${ }^{34,36}$ and the Project Viva cohort, ${ }^{4,9,33}$ one U.K.-based cohort, ${ }^{31}$ the Generation R cohort, ${ }^{27}$ and the Danish National Birth Cohort. ${ }^{17-20}$

- About 17 percent of participants in two ALSPAC cohort responded yes to experiencing financial difficulties, ${ }^{1,2}$ while $~ 26$ percent of participants in a third ALSPAC cohort indicated many financial 
difficulties. ${ }^{29}$ About 16 percent of participants were eligible for free healthcare in the Lifeways Cross-Generation cohort study. ${ }^{6,30}$

- One $\mathrm{RCT}^{8}$ and three cohorts ${ }^{3,32,38}$ did not report on income or employment status.

Table 1. Included trials and cohorts

\begin{tabular}{|c|c|c|}
\hline Trial/Cohort name & Outcome(s) & References \\
\hline \multicolumn{3}{|l|}{ Randomized Controlled Trials } \\
\hline Linköping (Sweden) & $\begin{array}{l}\text { Atopic dermatitis/eczema } \\
\text { Allergic rhinitis }\end{array}$ & 7 \\
\hline Tokyo (Japan) & $\begin{array}{l}\text { Atopic dermatitis/eczema } \\
\text { Food allergy } \\
\text { Allergic rhinitis } \\
\text { Asthma }\end{array}$ & 8 \\
\hline Stockholm and Uppsala (Sweden) & $\begin{array}{l}\text { Atopic dermatitis/eczema } \\
\text { Allergic rhinitis }\end{array}$ & 14 \\
\hline Guildford (U.K.) & Atopic dermatitis/eczema & 16 \\
\hline Bangkok (Thailand) & Atopic dermatitis/eczema & 37 \\
\hline San Diego (U.S.) & $\begin{array}{l}\text { Atopic dermatitis/eczema } \\
\text { Food allergy } \\
\text { Allergic rhinitis } \\
\text { Asthma }\end{array}$ & $34-36$ \\
\hline \multicolumn{3}{|l|}{ Non-Randomized Controlled Trials } \\
\hline Berlin (Germany) & Atopic dermatitis/eczema & 10 \\
\hline \multicolumn{3}{|l|}{ Prospective Cohort Studies } \\
\hline Osaka Maternal and Child Health Study (OMCHS) & Atopic dermatitis/eczema & $21-24,28$ \\
\hline $\begin{array}{l}\text { Kyushu Okinawa Maternal and Child Health Study } \\
\text { (KOMCHS) }\end{array}$ & Atopic dermatitis/eczema & 25,26 \\
\hline Rhea \& INfancia y Medio Ambiente (INMA) & Atopic dermatitis/eczema & 5 \\
\hline Generation R Study & $\begin{array}{l}\text { Atopic dermatitis/eczema } \\
\text { Asthma }\end{array}$ & 13,27 \\
\hline $\begin{array}{l}\text { Growing Up in Singapore Towards Healthy } \\
\text { Outcomes (GUSTO) }\end{array}$ & $\begin{array}{l}\text { Atopic dermatitis/eczema } \\
\text { Allergic rhinitis }\end{array}$ & 15 \\
\hline
\end{tabular}




\begin{tabular}{|c|c|c|}
\hline Trial/Cohort name & Outcome(s) & References \\
\hline $\begin{array}{l}\text { Avon Longitudinal Study of Parents and Children } \\
\text { (ALSPAC) }\end{array}$ & $\begin{array}{l}\text { Atopic dermatitis/eczema } \\
\text { Food allergy } \\
\text { Allergic rhinitis } \\
\text { Asthma }\end{array}$ & $1,2,12,29$ \\
\hline $\begin{array}{l}\text { Norwegian Mother and Child } \\
\text { Cohort Study (MoBa) }\end{array}$ & $\begin{array}{l}\text { Atopic dermatitis/eczema } \\
\text { Allergic rhinitis } \\
\text { Asthma }\end{array}$ & 3 \\
\hline Project Viva & $\begin{array}{l}\text { Atopic dermatitis/eczema } \\
\text { Food allergy } \\
\text { Allergic rhinitis } \\
\text { Asthma }\end{array}$ & $4,9,33$ \\
\hline Danish National Birth Cohort (DNBC) & $\begin{array}{l}\text { Allergic rhinitis } \\
\text { Asthma }\end{array}$ & $17-20$ \\
\hline Lifeways Cross-Generation Cohort Study & Asthma & 6,30 \\
\hline $\begin{array}{l}\text { Type } 1 \text { Diabetes Prediction and Prevention (DIPP) } \\
\text { Project }\end{array}$ & Asthma & 38 \\
\hline $\begin{array}{l}\text { Prevention and Incidence of Asthma and Mite } \\
\text { Allergy (PIAMA) }\end{array}$ & Asthma & 32 \\
\hline New York City (U.S.) and Krakow (Poland) & Atopic dermatitis/eczema & 11 \\
\hline Aberdeen (U.K.) & $\begin{array}{l}\text { Atopic dermatitis/eczema } \\
\text { Allergic rhinitis } \\
\text { Asthma }\end{array}$ & 31 \\
\hline
\end{tabular}

\section{Interventions/Exposures}

The intervention/exposure for this review was intentionally broad and included foods or food groups that may or may not be considered allergens. This review, however, did not assess the relationship between specific nutrients or dietary supplements and allergies and atopic allergic diseases. Studies included in this body of evidence assessed maternal diet during pregnancy and/or lactation in three main ways:

\section{Avoidance diet}

Six RCTs s, $^{7,14,16,34-37}$ and one NRCT ${ }^{10}$ focused on maternal avoidance of specific foods/food groups including:

- Cow milk products ${ }^{8,16,37}$

- Cow milk products and egg 7,10,14

- Cow milk products, egg, peanut, soy, and wheat ${ }^{34-36}$

\section{Dietary patterns}


Dietary patterns (DP) were assessed using an 1) index/score analysis or a 2) factor analysis and principal component analysis. A description of the studies categorized by the method used to measure dietary patterns is included below:

- Five articles representing four cohorts used the following indices/scores summarized below:
- Mediterranean DP5
- Diet quality score ${ }^{27}$
- Mediterranean diet score ${ }^{1}$
- Dietary Inflammatory Index (DII) ${ }^{9}$
- Healthy Eating Index $(\mathrm{HEI})^{6}$

- Three articles assessed dietary patterns using factor analysis or principal component analysis:

- Healthy DP, Western DP, and Japanese DP21

- Vegetable, fruit and white rice DP, Seafood and noodles DP, Pasta, cheese and processed meat DP ${ }^{15}$

- Health conscious DP, Traditional DP, Processed DP, Vegetarian DP and Confectionary DP ${ }^{29}$

\section{Food/beverage consumption levels}

Twenty-two articles from 13 cohort studies compared the association between different consumption levels of a variety of different foods and beverages and risk of atopic outcomes, including:

- Dairy products ${ }^{4,5,18,22,26,28,32,38}$

○ Yogurt and fermented milk products $3,18,22,26,28,32$

- Peanuts 4,17

- Nuts, $5,17,32$

- Wheat/cereals $\mathrm{s}^{4,5,31}$

- Fish $^{5,11,13,19,24,25,28,30-32,38}$

- Eggs 4,32

- Soybean ${ }^{4,12}$

- Foods not commonly considered to be allergens (as reported by the studies)
- Fruits $2,5,23,31,32$
- Vegetables $2,5,23,30-32$
- Sugar-sweetened beverages 20,33
- Artificially-sweetened soft drinks ${ }^{20}$
- Margarine/oil/fats ${ }^{31,38}$
- Meat ${ }^{5,24,25,28,38}$

\section{Time point of intervention/exposure}

The RCTs and NRCT began the avoidance diets at different time points:

- Early third trimester through the end of pregnancy ${ }^{7,14}$ or through early 
lactation $34-36$

- Late third trimester through early lactation $8,10,16$

- After delivery through early lactation ${ }^{37}$

Most of the cohort studies administered a food frequency questionnaire (FFQ) or 24h recall at a single time point during pregnancy. ${ }^{1-3,5,6,11-13,15,17-32}$ In the Project Viva cohort, dietary data were collected at multiple time points during pregnancy and assessed at individual time points (e.g. by trimester) or combined in the analysis. ${ }^{4,9,33}$ In one cohort study, maternal dietary data were collected at a single time point during lactation by a FFQ. ${ }^{38}$

\section{Outcome}

Food allergy was defined as a diagnosis based on either the gold standard of a double-blind, placebo-controlled oral food challenge, ${ }^{12}$ or as parental report of clinical history together with blood IgE levels $\geq 0.35 \mathrm{kU} / \mathrm{L}$ and/or skin prick test wheal $\geq 3$ $\mathrm{mm} .4,8,12,34-36$ Studies that reported food sensitization only, based solely on IgE levels or a skin prick test, were excluded.

Atopic dermatitis/eczema, allergic rhinitis, and asthma outcomes in this body of evidence were measured in three ways:

- Self-reported symptoms, prescription, or doctor diagnosis via a questionnaire ${ }^{1-}$ $5,8,9,13,15,17-29,31-33,38$

- Clinical examination $6,7,10,14,16,30,34-37$

- Hospital admission and prescription registries ${ }^{17-20}$

Because of the difficulty in diagnosing asthma during infancy and toddlerhood, only those studies that assessed asthma in children who were at least 2 years or older were included in this systematic review.

\section{Evidence synthesis - Food allergy}

With six articles, ${ }^{4,8,12,34-36}$ there is a smaller body of evidence available to examine the relationship between maternal diet during pregnancy and lactation and food allergies in the child.

\section{Cow milk products}

Pregnancy: A U.S.-based cohort study 4 showed no association between maternal consumption of milk during pregnancy in the first and second trimesters and risk of food allergy in the child.

Pregnancy and lactation: A Japan-based $\mathrm{RCT},{ }^{8}$ conducted in high-risk families, randomized mothers to consume hypoallergenic formula and avoid cow milk products during late pregnancy and lactation. Results showed no difference in the prevalence of overall allergies between children of the intervention and control group mothers and median blood IgE levels of infants did not differ between groups at 4 months of age.

Three additional articles from one U.S.-based trial ${ }^{34-36}$ assessed the effect of a prophylactic treatment, which included avoidance of cow milk, egg, and peanut during the third trimester and while lactating, on development of atopy in children from highrisk families. In addition to the intervention in the mothers, infants in the intervention group also received an intervention, which included the following: a) casein 
hydrolysate formula when supplementing human milk and during weaning, b) not started on solid food until 6 months of age, c) cow milk, corn, soy, citrus and wheat restriction until 12 months of age, and d) eggs, peanut, and fish restriction until 24 months of age. The mothers in the control group followed a standard diet during pregnancy and lactation their infants were supplemented with standard infant formula (Enfamil) during human milk feeding and weaning; solid foods were introduced to control group infants in accordance with standard guidance by the American Academy of Pediatrics. While the three articles from this $\mathrm{RCT}^{34-36}$ met the inclusion criteria and findings are reported in Table 2, they were not considered in evidence synthesis, grading, or when drawing conclusions because of confounding from the additional child intervention. The trial also had limitations regarding risk of bias due to randomization, attrition rate (38 percent in intervention vs. 13 percent in control group; $\mathrm{p}<0.001$ ), and non-blinding of the outcome assessors and participants.

Lactation: No studies assessed the relationship between maternal consumption of cow milk products during lactation and risk of food allergy in the child.

\section{Egg}

Pregnancy: A U.S.-based cohort study ${ }^{4}$ showed no association between maternal consumption of eggs during the first and second trimesters and risk of food allergy in the child.

Pregnancy and lactation: As described above (p. 31), three articles ${ }^{34-36}$ from a single U.S. trial met the inclusion criteria; however, they were not considered in evidence synthesis, grading, or when drawing conclusions.

Lactation: No studies assessed the relationship between maternal consumption of eggs during lactation and risk of food allergy in the child.

\section{Peanut, tree nut and seed}

Pregnancy: One prospective cohort study ${ }^{4}$ conducted in the U.S. showed no association between maternal peanut consumption during the first and second trimesters and risk of food allergy in the child.

No study assessed the relationship between maternal tree nut and seed consumption during pregnancy and risk of food allergy in the child.

Pregnancy and lactation: Three articles ${ }^{34-36}$ from a single U.S. trial met the inclusion criteria; however, they were not considered in evidence synthesis, grading, or when drawing conclusions for the reasons described above (p. 31).

Lactation: No study assessed the relationship between maternal peanut, tree nut and seed consumption during lactation and risk of food allergy in the child.

\section{Soy}

Pregnancy: Two prospective cohort studies, one based in the U.S. ${ }^{4}$ and another based in the U.K., ${ }^{12}$ showed no association between maternal soy consumption during pregnancy and risk of food allergy in the child. Note: The U.K. study assessed the association between maternal soybean meat consumption (data collected prospectively) during pregnancy and risk of peanut allergies diagnosed using a food challenge in the child. While the study also assessed the association between maternal peanut consumption and risk of peanut allergies in the child, this data was collected retrospectively and did not meet the inclusion-exclusion criteria. 
Pregnancy and lactation: Three articles ${ }^{34-36}$ from a single U.S. trial met the inclusion criteria; however, they were not considered in evidence synthesis, grading, or when drawing conclusions for the reasons described above (p. 31).

Lactation: There were no studies that assessed the relationship between maternal soy consumption during lactation and risk of food allergy in the child.

\section{Wheat}

Pregnancy: A U.S.-based cohort study ${ }^{4}$ showed no association between maternal wheat consumption during pregnancy in the first and second trimesters and risk of food allergy in the child.

Pregnancy and lactation: Three articles ${ }^{34-36}$ from a single U.S. trial met the inclusion criteria; however, they were not considered in evidence synthesis, grading, or when drawing conclusions for the reasons described above (p. 31).

Lactation: No studies assessed the relationship between maternal wheat consumption during lactation and risk of food allergy in the child.

\section{Other foods}

Pregnancy: No study assessed the relationship between maternal consumption of other foods not commonly considered to be allergens, during lactation and risk of food allergy in the child.

\section{Assessment of the evidence ${ }^{\mathrm{vi}}$}

\section{Soy}

The following conclusion statement was supported by two cohort studies ${ }^{4,12}$ and was graded "limited." The individual grading elements are discussed below.

"Limited evidence suggests no relationship between soybean consumed during pregnancy and risk of food allergy in the child."

As outlined and described below, the body of evidence examining soybean consumed during pregnancy and risk of food allergy in the child was assessed for the following elements used when grading the strength of evidence.

- Risk of bias (Table 8) was graded as limited as there were serious flaws in the design and conduct of the studies, which are described below:

- At least one of the key confounders was not controlled for in the U.S.-based cohort study ${ }^{4}$ and none of the key confounders were accounted for in the U.K.based study. ${ }^{12}$

- Dietary data reflected dietary intake at a single time point during pregnancy and may not have reflected the intake during the entire period of pregnancy. In the U.K. study, the exposure status (i.e., soybean meat) and methods used to assess exposure were not clearly defined. Further, the U.K. study did not assess the impact of consumption of a particular food with regard to the development of

\footnotetext{
${ }^{v i}$ A detailed description of the methodology used for grading the strength of the evidence is available on the NESR website: https://nesr.usda.gov/2020-dietary-guidelines-advisory-committee-systematicreviews and in Part $\mathrm{C}$ of the following reference: Dietary Guidelines Advisory Committee. 2020. Scientific Report of the 2020 Dietary Guidelines Advisory Committee: Advisory Report to the Secretary of Agriculture and the Secretary of Health and Human Services. U.S. Department of Agriculture, Agricultural Research Service, Washington, DC.
} 
allergies to that particular food.

- One study assessed outcomes using non-validated or unreliable measures (e.g., parent report of a child's symptoms or parent's report of a physician diagnosis).

- None of the included studies had a pre-registered data analysis plan. Further, there was a likelihood of selective reporting in the U.K. study.

- Consistency was graded as strong since both studies consistently reported no association between soy consumption during pregnancy and risk of food allergy in the child.

- Directness was graded as limited. Although the U.K. study reported the association between maternal soybean meat consumption during pregnancy and risk of food allergy in the child, it was not the primary purpose of the study. While the U.S.based study focused on addressing the systematic review question, these data were from a large cohort study (Project Viva) that addressed associations between different exposures and outcomes.

- Precision was graded as limited. Even though both studies had a moderate to large number of participants $\left(11,352\right.$ participants ${ }^{12}$ and approximately 600 participants $\left.^{4}\right)$, there were only two cohort studies included in this body of evidence. However, neither of them unduly affected the findings of this systematic review.

- Generalizability was graded as limited. Although one of the studies was conducted in the U.S., the participants were predominantly White and were from mid-to-high SES. In the U.K.-based study, the authors provided minimal information on the race/ethnicity or other demographic characteristics.

\section{Other foods}

The bodies of evidence that assessed the relationship between:

- Lower or restricted consumption of cow milk products during pregnancy, or during pregnancy and lactation,

- Peanuts, eggs, or wheat consumed during pregnancy,

and risk of food allergy in the child were rated 'grade not assignable' for one or more of the following reasons:

- a very small body of evidence, usually less than two studies,

- heterogeneity in the findings,

- serious flaws in the design and conduct of the study, and

- limited generalizability of the findings to the U.S. population.

\section{Evidence synthesis - Atopic dermatitis/eczema}

With 26 articles, ${ }^{1,3-5,7,8,10,11,13-16,21-29,31,34-37}$ there is a substantial body of evidence available to examine the relationship between maternal diet during pregnancy and/or lactation and atopic dermatitis/eczema in the child (Table 3).

\section{Cow milk products}

Pregnancy: Seven studies, including two RCTs ${ }^{7,14}$ and five prospective 
cohorts, $4,5,22,26,28$ assessed the relationship between cow milk products consumed during pregnancy and risk of atopic dermatitis/eczema. The RCTs were specifically designed to assess the relationship between the avoidance of cow milk products during pregnancy and atopic dermatitis/eczema in the child, whereas the cohort studies assessed the association between consumption of cow milk products and atopic dermatitis/eczema. As mentioned elsewhere, the RCTs primarily recruited highrisk families and this was not the case with prospective cohort studies.

None of the studies showed an association between maternal cow milk product consumption and atopic dermatitis/eczema. The only exception was a sub-group analysis in a Project Viva cohort study ${ }^{4}$ that showed that maternal milk consumption during the first trimester was associated with a lower risk of atopic dermatitis only among children whose parents had atopy. However, there was no association between second trimester cow milk consumption and atopic dermatitis risk in child, irrespective of parental atopy.

Pregnancy and lactation: The three articles ${ }^{34-36}$ from a single U.S. trial met the inclusion criteria. The findings are presented in Table 3 . However, they were not considered in evidence synthesis, grading, or when drawing conclusions for the reasons described above (p. 31).

Lactation: One Thailand-based $\mathrm{RCT}^{37}$ with 62 participants showed that infants born to the mothers who were randomized to a dairy product-restricted diet (vs. usual diet) had lower incidence of atopic dermatitis at 4 months of age.

\section{Fermented milk and yogurt}

Pregnancy: Two of the three cohort studies ${ }^{3,26}$ showed an association between higher fermented milk and yogurt consumed during pregnancy and risk of atopic dermatitis/eczema. Specifically, in a Japanese study with a sample size of 1,354 participants, Miyake et al $(2014)^{26}$ noted that higher maternal yogurt intake during pregnancy was associated with a lower odds of physician-diagnosed atopic eczema in children aged 23 to 29 months. In a Norwegian cohort with 40,614 participants, Bertelsen et $\mathrm{al}^{3}$ showed that higher maternal probiotic milk and yogurt consumption (vs. no consumption) was associated with a reduced risk of atopic eczema in the infant at 6 months of age. There was no association with eczema in the child at 18 months of age. In a stratified analysis, the authors compared no intake vs. child intake only vs. mother intake only vs. mother and child intake only and risk of eczema at 18 months and noted the following:

- Maternal intake only (vs. no intake) was associated with an increased risk of atopic eczema at 18 months in children (RR: 1.08, 95\% Cl: 1.01, 1.15)

- Mother and child intake (vs. no intake) was associated with statistically significant lower eczema risk at 18 months (RR: $0.93,95 \% \mathrm{Cl}: 0.86,1.00$ )

An earlier study published by Miyake et al ${ }^{22}$ in 2010 showed that yogurt consumption during pregnancy was not associated with the risk of atopic dermatitis in the child. While the findings of this cohort study were not statistically significant, the directionality was consistent with the overall findings of the other two studies. ${ }^{26}{ }^{3}$.

None of the studies assessed an association between maternal fermented milk and yogurt consumption during lactation and risk of atopic dermatitis/eczema.

\section{Egg}


Pregnancy: Four studies, including two RCTs ${ }^{7,14}$ and two cohort studies, ${ }^{4,28}$ assessed the relationship between maternal egg consumption/avoidance during pregnancy and risk of atopic dermatitis. Specifically, the two trials ${ }^{7,14}$ conducted in Sweden that randomized mothers to avoid egg consumption during pregnancy (in addition to avoiding milk products) showed no relationship between the avoidance diet and the risk of atopic dermatitis/eczema in the child. These findings were in line with the results of the U.S.-based cohort study ${ }^{4}$ and a Japanese cohort study, ${ }^{28}$ which both showed no association between eggs consumed during pregnancy and risk of atopic dermatitis in the child at $3-4$ months of age ${ }^{28}$ or 7.9 years. ${ }^{4}$

Pregnancy and lactation: A German NRCT (baseline $n=150$ ) showed that maternal avoidance of egg (in addition to avoiding cow milk products) during pregnancy and lactation was not associated with the risk of atopic dermatitis in the child during the first year of life (6 months, or 12 months). ${ }^{10}$

The three articles ${ }^{34-36}$ from a single U.S. trial met the inclusion criteria; however, they were not considered in evidence synthesis, grading, or when drawing conclusions for the reasons described above (p. 31).

Lactation: There were no other studies that assessed the relationship between maternal egg consumption during lactation and risk of atopic dermatitis/eczema in the child.

\section{Fish}

Pregnancy: Two of the seven cohort articles showed that higher consumption of fish during pregnancy was associated with a lower risk of atopic dermatitis/eczema in the child. Specifically, a U.K.-based cohort study ${ }^{31}$ with a baseline sample size of 1,751 participants showed that higher maternal total fish consumption during pregnancy was associated with a lower risk of doctor-confirmed eczema in 5 year old children. Similarly, a cohort study ${ }^{11}$ conducted in the Poland and the U.S. showed that higher maternal fish intake was associated with a lower risk of atopic dermatitis/eczema during the first year of life. Five other articles (from 4 cohorts) showed no association with the risk of atopic dermatitis during the first 2 years of life. 5,13,24,25,28 Three of these were conducted in Japan and two were conducted in a European population and all had at least 1,000 participants at baseline.

Lactation: No studies assessed the relationship between maternal fish consumption during lactation and risk of atopic dermatitis/eczema in the child.

\section{Peanut, tree nut and seed}

Pregnancy: Two studies, a U.S.-based cohort ${ }^{4}$ (baseline $n=2,128$ ) and a study based in Spain and Greece ${ }^{5}$ (baseline $n=2,984$ ), assessed the association between maternal peanut/nut consumption during pregnancy and risk of atopic dermatitis/eczema in the child and neither study showed an association. Of note, the Spain/Greece-based cohort study assessed nut consumption along with fruit consumption.

No studies showed an association between maternal tree nut and seed consumption during pregnancy and risk of atopic dermatitis/eczema in the child.

Pregnancy and lactation: Three articles ${ }^{34-36}$ from a single U.S. trial met the inclusion criteria; however, they were not considered in evidence synthesis, grading, or when drawing conclusions for the reasons described above (p. 31).

Lactation: None of the studies assessed the relationship between maternal peanuts, 
tree nuts, or seed consumption during lactation and risk of atopic dermatitis/eczema in the child.

\section{Soy}

Pregnancy: The only prospective cohort study ${ }^{4}$ that assessed the relationship between maternal soybean consumption during pregnancy and risk of atopic dermatitis/eczema in the child showed no association.

Pregnancy and lactation: The three articles ${ }^{34-36}$ from a single U.S. trial met the inclusion criteria; however, they were not considered in evidence synthesis, grading, or when drawing conclusions for the reasons described above (p. 31).

Lactation: No other studies assessed the relationship between maternal soybean consumption during lactation and risk of atopic dermatitis/eczema in the child.

\section{Wheat}

Pregnancy: One U.S.-based cohort study ${ }^{4}$ showed that maternal wheat consumption during the second trimester was associated with a lower risk of atopic dermatitis in the child at 7.9 years. However, there was no association between first trimester maternal wheat consumption and risk of atopic dermatitis/eczema in the child. A European cohort study ${ }^{5}$ conducted in Spain and Greece showed no association between maternal cereal consumption and risk of atopic dermatitis/eczema in the first year of life.

Pregnancy and lactation: Three articles ${ }^{34-36}$ from a single U.S. trial met the inclusion criteria; however, they were not considered in evidence synthesis, grading, or when drawing conclusions for the reasons described above (p. 31).

Lactation: None of the studies assessed the relationship between maternal wheat consumption during lactation and risk of atopic dermatitis/eczema in the child.

\section{Dietary patterns}

Pregnancy: Six articles from five studies ${ }^{1,5,15,21,27,29}$ assessed the association between maternal DPs during pregnancy and risk of atopic dermatitis/eczema in the child. As described below, the studies assessed a variety of DPs:

1. Healthy DP, Western DP, and Japanese DP21

2. Mediterranean DP5

3. Diet quality score ${ }^{27}$

4. Vegetable, fruit and white rice DP, Seafood and noodles DP, Pasta, cheese and processed meat $\mathrm{DP}^{15}$

5. Mediterranean diet score ${ }^{1}$

6. Health conscious DP, Traditional DP, Processed DP, Vegetarian DP and Confectionary DP29

Despite the heterogeneity in the DPs, none of the maternal DPs during pregnancy were associated with the risk of atopic dermatitis/eczema in the child.

Lactation: There were no studies that assessed the relationship between maternal DPs consumed during lactation and atopic dermatitis/eczema in the child.

\section{Other foods}

Pregnancy: Five articles from three cohort studies assessed the relationship between 
maternal consumption of other foods not commonly considered to be allergens, such as meat, ${ }^{5,24,25,28}$ vegetables, ${ }^{5,23}$ and fruits ${ }^{5,23}$ during pregnancy and atopic

dermatitis/eczema risk and noted the following:

- Meat: A Japanese cohort ${ }^{28}$ with a baseline sample size of 1,002 participants showed that higher consumption of meat was associated with an increased risk of atopic dermatitis/eczema in the child; however, three other studies ${ }^{5,24,25}$ (including one study from the same Japanese cohort ${ }^{24}$ ) showed no association.

- Vegetables: Of the two cohorts that assessed the association between maternal vegetable consumption and atopic dermatitis/eczema, the Japanese cohort ${ }^{23}$ (baseline $n=1,002$ ) showed that a greater adherence to green and yellow vegetables was associated with a lower risk of atopic dermatitis/eczema in children at 16-24 months. However, intake of total vegetables and other vegetables was not associated with the risk of atopic dermatitis/eczema. Similarly, there were no associations between maternal vegetable consumption during pregnancy and atopic dermatitis/eczema risk in children at approximately 9-14 months in a European cohort. ${ }^{5}$

- Fruits: The same cohorts described in the above vegetables section also assessed the relationship between maternal fruit consumption during pregnancy and atopic dermatitis/eczema risk in the child. ${ }^{5,23}$ While the Japanese cohort ${ }^{23}$ showed that higher citrus fruit consumption was associated with a lower atopic dermatitis/eczema risk, no association was observed with maternal total fruit and apple consumption. Similarly, a European cohort study during pregnancy ${ }^{5}$ found no association between fruit consumption (which was jointly assessed with nut consumption) and the risk of atopic dermatitis/eczema in the child.

\section{Assessment of the evidence ${ }^{\text {vii }}$}

\section{Cow milk products}

The following conclusion statement was supported by two RCTs ${ }^{7,14}$ and five cohort studies $^{4,5,22,26,28}$ and was graded "moderate." Below, the individual grading elements are discussed separately for every study design.

"Moderate evidence indicates that lower or restricted consumption of cow milk products during pregnancy does not reduce the risk of atopic dermatitis/eczema in the child."

As outlined and described below, the body of evidence examining consumption of cow milk products during pregnancy and risk of atopic dermatitis/eczema in the child was assessed for the following elements used when grading the strength of evidence.

- Risk of bias was graded as moderate for the RCTs and limited for the cohort studies.

- RCTs (Table 6): The two RCTs included in this body of evidence had notable flaws, as described below:

\footnotetext{
vii A detailed description of the methodology used for grading the strength of the evidence is available on the NESR website: https://nesr.usda.gov/2020-dietary-guidelines-advisory-committee-systematicreviews and in Part $\mathrm{C}$ of the following reference: Dietary Guidelines Advisory Committee. 2020. Scientific Report of the 2020 Dietary Guidelines Advisory Committee: Advisory Report to the Secretary of Agriculture and the Secretary of Health and Human Services. U.S. Department of Agriculture, Agricultural Research Service, Washington, DC.
} 
- Falth-Magnusson et $\mathrm{al}^{7}$ reported issues with adherence to the assigned intervention. There was differential adherence to the intervention: 79 of the 104 mothers randomized to the intervention group adhered to the cow milk- and egg-free diet and provided follow-up data on their children at 18 months, while 102 of the 108 women randomized to control group adhered to the standard diet and provided follow-up data on their children at 18 months.

- Lilja et al ${ }^{14}$ reported potential issues with randomization. Mothers in the intervention group reported significantly higher lgE levels and greater prevalence of atopic eczema when compared to the control group. There were deviations from the intended intervention, with some of the women in the intervention group continuing with the intervention through lactation. There were challenges with selective reporting of results. Although the outcome was assessed throughout infancy and early childhood, atopic dermatitis was not reported during the earlier time points.

- Prospective cohort studies (Table 8): Similar to the RCTs, the cohort studies had serious flaws in the design and conduct of the studies:

- At least one of the key confounders was not controlled for in all of the included studies.

- Dietary data reflected dietary intake at a single time point during pregnancy and may not have reflected the intake during the entire period of pregnancy.

- Outcome data were self-reported and potentially subjective as they were reported by the participants who were aware of the exposure.

- Studies included in this body of evidence had co-exposures that may have been related to both exposure and outcome.

- None of the included studies had a pre-registered data analysis plan.

- Consistency was graded strong for RCTs and moderate for prospective cohort studies.

- RCTs: Both RCTs ${ }^{7,14}$ showed no relationship between avoiding cow milk product during pregnancy and risk of atopic dermatitis/eczema.

- Prospective cohort studies: All five cohort articles, $4,5,22,26,28$ reported no association between maternal cow milk product consumption and risk of atopic dermatitis/eczema. The only exception was a sub-group analysis in the U.S.based study that showed that higher consumption of cow milk products was associated with a lower risk of atopic dermatitis/eczema in the child. Because of this inconsistency, this grading element for prospective cohort studies was rated moderate.

- Directness was graded moderate for the RCTs and limited for prospective cohort studies.

- RCTs: Both trials ${ }^{7,14}$ were designed to assess the relationship between maternal cow milk product consumption and development of atopic diseases in infants and toddlers up to 18 months of age. In addition to the avoidance of cow milk products, the intervention group was also asked to avoid eggs. Hence, the findings might not have been attributable to cow milk product avoidance only. 
For this reason, directness was graded moderate.

- Prospective cohort studies: While the articles ${ }^{4,5,22,26,28}$ included in this body of evidence focused on addressing the systematic review question considered, the data for these articles were from larger cohort studies that addressed associations between different exposures/outcomes.

- Precision was graded moderate for both RCTs and prospective cohort studies.

- RCTs: Precision was considered moderate because both trials had a reasonable sample size (baseline $n=171^{14}$ and $212,{ }^{7}$ respectively). However, power calculations were not reported in either of the studies. Neither of the studies unduly influenced the findings of this systematic review and removing a single study from this body of evidence would not likely change the conclusions.

- Prospective cohort studies: Precision for the prospective cohort studies was also considered moderate because there were an adequate number of studies that were sufficiently powered to assess the association. Baseline sample size included at least 1,000 participants in all these cohorts. No single article unduly affected the findings of this systematic review and removing one study would not impact the overall conclusions.

- Generalizability was graded limited for both RCTs and prospective cohort studies.

- RCTs: Both trials were conducted in Sweden in the 1980s. However, the studies provided very minimal information on age, race/ethnicity and other SES characteristics and thus the applicability of the findings to the U.S. population is unclear. Given the dearth of information, generalizability for RCTs was graded limited.

- Prospective cohort studies: For two of the five cohorts (corresponding to three articles $^{22,26,28}$ ), 100 percent of participants were from Japan. Chatzi et al ${ }^{5}$ combined findings from two cohort studies, one based in Spain and another one in Greece. However, the race/ethnicity of the participants was not reported. The only U.S.-based study ${ }^{4}$ included in this body of evidence noted that most of the participants were White and were of moderate-to-high SES. Based on the characteristics of the participants in the cohorts, it is unclear if the findings would be representative of the U.S. mothers and hence generalizability was graded limited.

\section{Egg}

The following conclusion statement was supported by two RCTs s $^{7,14}$ and two cohorts ${ }^{4,28}$ and was graded moderate. Below, the individual grading elements are discussed separately for every study design.

"Moderate evidence indicates that lower or restricted consumption of egg during pregnancy does not reduce the risk of atopic dermatitis/eczema in the child."

As outlined and described below, the body of evidence examining consumption of eggs during pregnancy and risk of atopic dermatitis/eczema in the child was assessed for the following elements used when grading the strength of evidence.

(Note: The same RCTs ${ }^{7,14}$ contributed to both (cow milk products and egg) bodies of evidence. Except for consistency, the individual grading elements for the RCTs are not discussed below and the readers are referred to the previous section on cow milk products.) 
- Risk of bias (Table 6, Table 8) was graded moderate for the RCTs and limited for the prospective cohort studies.

- Prospective cohort studies: There were serious flaws in the conduct of these studies, ${ }^{4,28}$ including lack of accounting of all the key confounders, subjective outcome reporting, a single point of exposure assessment during pregnancy, and lack of a pre-registered data analysis plan.

- Consistency was graded strong for the RCTs and prospective cohort studies.

- RCTs: There was a strong consistency in the findings in that both RCTs ${ }^{7,14}$ showed no relationship between mother's avoidance of eggs during pregnancy and risk of atopic dermatitis/eczema.

- Prospective cohort studies: Consistency for the prospective cohort studies ${ }^{4,28}$ was considered to be strong as both studies showed no association between egg consumption and the risk of atopic dermatitis/eczema. These findings were also consistent with the RCTs findings.

- Directness was graded moderate for the RCTs and limited for the prospective cohort studies.

- Prospective cohort studies: While the articles ${ }^{4,28}$ included in this body of evidence focused on addressing the current systematic review question, the data for these articles were from larger cohort studies that addressed associations between different exposures and outcomes.

- Precision was graded moderate for the RCTs and limited for the prospective cohort studies.

- Prospective cohort studies: Although the baseline sample size included at least 1,000 participants, there were only two cohort studies in this body of evidence. However, neither of them unduly affected the findings of this systematic review.

- Generalizability for the RCTs and the prospective cohort studies was graded limited.

- Prospective cohort studies: The U.S.-based study ${ }^{4}$ included in this body of evidence noted that most of the participants were White and were of moderateto-high SES. One other cohort study was conducted in Japan. In this study, more than two-thirds of the participants had $\geq 13$ years of education and most had moderate-to-high SES. Based on the characteristics of the participants included in the cohort, it is unclear if the findings would be representative of U.S. mothers.

\section{Fish}

The following conclusion statement was supported by six prospective cohort studies $5,11,13,24,25,28,31$ and was graded "limited." The individual grading elements are discussed below.

"Limited evidence suggests that fish consumed during pregnancy does not increase the risk of atopic dermatitis/eczema in the child."

As outlined and described below, the body of evidence examining consumption of fish during pregnancy and risk of atopic dermatitis/eczema in the child was assessed for 
the following elements used when grading the strength of evidence.

- Risk of bias (Table 8) was graded as limited as there were serious flaws in the design and conduct of the studies, which are described below:

- At least one of the key confounders was not controlled for in all of the included studies.

- Dietary data reflected dietary intake at a single time point during pregnancy and may not have reflected the intake during the entire period of pregnancy.

- Outcome data were self-reported and potentially subjective as they were reported by the participants who were aware of the exposure.

- Studies included in this body of evidence had co-exposures that may have been related to both exposure and outcome.

- Two studies ${ }^{5,11}$ in this body of evidence had possible selection bias issues and also had a potential for bias in classification of exposures.

- None of the included studies had a pre-registered data analysis plan.

- Consistency for this body of evidence was considered to be limited. Two ${ }^{11,31}$ of the seven articles reported that an increased consumption of fish during pregnancy was associated with a lower risk of atopic dermatitis and five articles ${ }^{5,13,24,25,28}$ (representing four cohorts) showed no association. The inconsistencies in findings across studies can be partly attributed to the methodological limitations that were noted in this body of evidence, including bias due to classification of exposure, deviations from intended exposures, and missing outcome data.

- Directness was graded as limited. While the articles $5,11,13,24,25,28,31$ included in this body of evidence focused on addressing the systematic review question, the data for these articles were from larger cohort studies that addressed associations between different exposures and outcomes.

- Precision was graded as moderate because there was an adequate number of studies that were sufficiently powered to assess the association. Sample sizes of most of the included studies in this body of evidence were at least 1,000 participants at baseline, with the exception of one study ${ }^{11}$ that included 469 participants. No single article unduly affected the overall findings of this systematic review and removing one study would not impact the overall conclusions.

- Generalizability was graded limited. Two of the six cohorts (corresponding to three articles ${ }^{24,25,28}$ ) were conducted in Japan. Chatzi et al ${ }^{5}$ combined findings from two cohort studies, one based in Spain and another one in Greece. However, the race/ethnicity of the participants was not reported. Two other European cohorts ${ }^{13,31}$ were included in this body of evidence. Specifically, Leermakers et $\mathrm{al}^{13}$ included 100 percent Dutch mothers from the Generation R cohort, noted that approximately 65 percent of the participants had 'higher' education. In the U.K.-based cohort, Willers et $\mathrm{al}^{31}$ did not report race/ethnicity, but noted that mothers left full-time education at a median age of 18.5 years. Finally, the cohort study conducted in the U.S. and Poland noted that women had 15.7 years of education, but did not provide other characteristics including race/ethnicity. Based on the characteristics of the participants in the cohorts, it is unclear if the findings would be generalizable to the U.S. mothers and hence generalizability was graded limited. 


\section{Dietary patterns}

The following conclusion statement was supported by five prospective cohort studies $1,5,15,21,27,29$ and was graded "limited." The individual grading elements are discussed below.

"Limited evidence suggests that dietary patterns during pregnancy are not associated with risk of atopic dermatitis/eczema in the child."

As outlined and described below, the body of evidence examining dietary patterns during pregnancy and risk of atopic dermatitis/eczema in the child was assessed for the following elements used when grading the strength of evidence.

- Risk of bias (Table 8) was graded as limited for the cohort studies because of the serious flaws in the design and conduct of the studies:

- At least one of the key confounders was not controlled for in all of the included studies.

- Dietary data reflected dietary intake at a single time point during pregnancy and may not have reflected the intake during the entire period of pregnancy.

- Outcome data were self-reported and potentially subjective as they were reported by the participants who were aware of the exposure.

- Studies included in this body of evidence had co-exposures that may have been related to both exposure and outcome.

- One study 5 in this body of evidence had possible selection bias issues and potential for bias in classification of exposures.

- None of the included studies had a pre-registered data analysis plan.

- Consistency was graded as strong as all of the included articles consistently showed no association between maternal dietary patterns during pregnancy and risk of atopic dermatitis/eczema in the child.

- Directness was considered to be limited. While the articles included in this body of evidence focused on addressing the systematic review question, the data for these articles are from larger cohort studies that addressed associations between different exposures and outcomes.

- Precision was graded as moderate because of the adequate number of studies that were sufficiently powered to assess the association. Baseline sample sizes included at least 1,000 participants in most of the cohorts, except the Singapore-based cohort, ${ }^{15}$ which had a baseline sample size of 735 . No single article unduly affected the findings of this systematic review and removing one study would not impact the overall conclusions.

- Generalizability was graded as limited. Of the studies conducted in Europe, two were from the same U.K.-based ALSPAC cohort, ${ }^{1,29}$ one from Spain/Greece, ${ }^{5}$ and one from the Netherlands. ${ }^{27}$ The U.K.-based articles reported that most of the participants were White and possibly from mid-to-high SES. The other studies did not report the race/ethnicity of the mothers. The Singapore-based cohort study reported that all of the participants were Asian and the Japanese cohort study reporting that the 100 percent of the participants were from Japan. None of these studies were conducted in the U.S. Based on the characteristics of the participants in the cohorts, it is unclear if the findings would be representative of the U.S. 
mothers.

\section{Other foods}

The bodies of evidence that assessed the relationship between:

- Peanuts, soybean, wheat/cereal, yogurt and probiotic milk products, and other foods (not commonly considered allergens, including meat, vegetables, and fruits) consumed during pregnancy,

- Cow milk products and eggs restricted during both pregnancy and lactation,

- Cow milk products restricted during lactation,

and risk of atopic dermatitis/eczema in the child were rated 'grade not assignable' for one or more of the following reasons:

- a very small body of evidence, usually less than two studies,

- heterogeneity in the findings,

- serious flaws in the design and conduct of the study, and

- limited generalizability of the findings to the U.S. population

\section{Evidence synthesis - Allergic rhinitis}

With 16 articles, $1,3,4,7,8,14,15,17-20,29,31,34-36$ there is a modest body of evidence available to examine the relationship between maternal diet during pregnancy and lactation and allergic rhinitis in the child.

\section{Cow milk products}

Pregnancy: Five studies, including two RCTs ${ }^{7,14}$ and three prospective cohort studies, ${ }^{3,4,18}$, assessed the relationship between consumption of cow milk products during pregnancy and risk of atopic dermatitis in the child. The RCTs were specifically designed to assess the relationship between the avoidance of cow milk products during pregnancy and allergic rhinitis, whereas the cohort studies assessed the association between maternal consumption of cow milk products during pregnancy and allergic rhinitis in the child. Two RCTs showed that there was no relationship between maternal avoidance of cow milk during pregnancy and risk of allergic rhinitis in the child. A large Norway-based cohort study ${ }^{3}$ (baseline $n=40,614$ ) showed that higher consumption of probiotic milk and yogurt during pregnancy was associated with a lower risk of rhinoconjunctivitis. Similarly, the U.S.-based cohort study ${ }^{4}$ showed that higher intake of milk during the first trimester was associated with a lower risk of allergic rhinitis in the child and this association was primarily seen among those without parental atopy. There was no association between maternal milk consumption during the second trimester and risk of allergic rhinitis. On the other hand, a Denmarkbased cohort study ${ }^{18}$ (baseline $n=61,909$ ) noted that high intake of low-fat yogurt ( $>1$ serving per day vs. none) during pregnancy was associated with a greater risk of selfreported allergic rhinitis diagnosis in the child.

Pregnancy and lactation: A Japanese $\mathrm{RCT},{ }^{8}$ conducted in high-risk families, randomized mothers to consume hypoallergenic formula and asked mothers in the intervention group to avoid cow milk products during late pregnancy and lactation. Results showed no difference in the prevalence of overall allergies (including allergic 
rhinitis, asthma and atopic eczema) between children of the intervention and control group mothers and there was no difference in allergic rhinitis between groups at any age. The three articles ${ }^{34-36}$ from a single U.S. trial met the inclusion criteria. The findings from these studies are presented in Table 4. However, they were not considered in evidence synthesis, grading, or when drawing conclusions for the reasons described above (p. 31).

Lactation: No other studies assessed the relationship between maternal consumption of cow milk products during lactation and risk of allergic rhinitis in the child.

\section{Egg}

Pregnancy: Three studies, including two RCTs ${ }^{7,14}$ and one cohort study, ${ }^{4}$ assessed the relationship between maternal egg consumption/avoidance during pregnancy and risk of allergic rhinitis in the child. Specifically, the two trials ${ }^{7,14}$ conducted in Sweden that randomized mothers to avoid consumption of eggs during pregnancy (in addition to avoiding milk products) showed no relationship between an avoidance diet and risk of allergic rhinitis. These findings were in line with the results of the U.S.-based cohort study, ${ }^{4}$ which also showed no association between maternal consumption of eggs during pregnancy and risk of allergic rhinitis in the child at 7.9 years.

Pregnancy and lactation: Three articles ${ }^{34-36}$ from a single U.S. trial met the inclusion criteria; however, they were not considered in evidence synthesis, grading, or when drawing conclusions for the reasons described above (p. 31).

Lactation: No other studies assessed the relationship between maternal consumption of eggs during lactation and risk of allergic rhinitis in the child.

\section{Fish}

Pregnancy: Two cohort studies ${ }^{19,31}$ assessed the association between maternal fish consumption during pregnancy and risk of allergic rhinitis. In the U.K.-based cohort study, ${ }^{31}$ the authors reported that higher oily fish consumption ( $\geq 1$ portion per week vs. never) was associated with a lower risk of doctor confirmed hay fever (allergic rhinitis). However, maternal oily fish consumption during pregnancy was not associated with hay fever (allergic rhinitis) in the child when the outcome was reported as "current hay fever medication" or "ever hay fever". In a large Denmark-based cohort study, ${ }^{19}$ maternal fish intake during pregnancy was not associated with "ever prescribed" allergic rhinitis (as described by the study). However, lower fish intake, when compared to higher fish intake, during pregnancy was associated with a reduced risk of self-reported allergic rhinitis diagnosis in the child ( $P$ for trend=0.01).

Lactation: None of the studies assessed the association between maternal fish consumption during lactation and risk of allergic rhinitis in the child.

\section{Peanut, tree nut and seed}

Pregnancy: A U.S.-based cohort ${ }^{4}$ (baseline $n=2,128$ ) and a Denmark-based cohort study ${ }^{17}$ (baseline $n=61,908$ ) assessed the association between maternal peanut consumption during pregnancy and risk of allergic rhinitis in the child. The U.S.-based cohort study showed no association between maternal peanut consumption during the first and second trimesters and allergic rhinitis risk. The Denmark-based cohort study ${ }^{17}$ reported no association between maternal peanut (and pistachio) consumption during pregnancy and self-reported allergic rhinitis diagnosis. However, maternal consumption of peanuts (and pistachios) (1 time per month and 2-3 times per month 
vs. never) during pregnancy was associated with lower odds of "ever prescribed" allergic rhinitis (as described in the study) in the child ( $P$ for trend=0.001).

The Denmark-based cohort study ${ }^{17}$ also assessed the association between maternal tree nut consumption and risk of allergic rhinitis in the child and found no association.

Pregnancy and lactation: The three articles ${ }^{34-36}$ from a single U.S. trial met the inclusion criteria; however, they were not considered in evidence synthesis, grading, or when drawing conclusions for the reasons described above (p. 31).

Lactation: None of the studies assessed an association between maternal peanut, tree nut, seed consumption during lactation and risk of allergic rhinitis in the child.

\section{Soy}

Pregnancy: The U.S.-based cohort study, ${ }^{4}$ found no association between maternal soy consumption during pregnancy and risk of allergic rhinitis in the child.

Pregnancy and lactation: Three articles ${ }^{34-36}$ from a single U.S. trial met the inclusion criteria; however, they were not considered in evidence synthesis, grading, or when drawing conclusions for the reasons described above (p. 31).

Lactation: None of the studies assessed the relationship between maternal soybean consumption during lactation and risk of allergic rhinitis in the child.

\section{Wheat}

Pregnancy: Bunyavanich et al, ${ }^{4}$ the U.S.-based cohort study, showed no association between maternal wheat consumption during both the first and second trimesters and risk of allergic rhinitis in the child in mid-childhood.

Pregnancy and lactation: The three articles ${ }^{34-36}$ from a single U.S. trial met the inclusion criteria; however, they were not considered in evidence synthesis, grading, or when drawing conclusions for the reasons described above (p. 31).

Lactation: None of the studies assessed the relationship between maternal wheat consumption during lactation and allergic rhinitis in the child.

\section{Dietary patterns}

Pregnancy: Three articles from two cohort studies ${ }^{1,15,29}$ assessed the association between maternal DPs and risk of allergic rhinitis in the child. As noted below, studies assessed a variety of DPs:

1. Vegetable, fruit, and white rice DP, Seafood and noodles DP, and Pasta, cheese and processed meat $\mathrm{DP}^{15}$

2. Mediterranean diet score ${ }^{1}$

3. Health conscious DP, Traditional DP, Processed DP, Vegetarian DP and Confectionary DP ${ }^{29}$

Despite the heterogeneity in the DPs, none of the maternal DPs were associated with the risk of allergic rhinitis in the child.

Lactation: There were no studies that assessed the relationship between maternal DPs during lactation and allergic rhinitis risk in the child.

\section{Other foods}

Pregnancy: A Denmark-based cohort study ${ }^{20}$ (baseline $n=60,465$ ) reported that higher 
consumption of artificially-sweetened carbonated soft drinks during pregnancy was associated with higher risk of self-reported childhood allergic rhinitis diagnosis compared to lower consumption ( $P$ for trend $=0.01$ ). No association was noted for other types of beverage consumption and allergic rhinitis risk in the child.

Lactation: There were no studies that assessed the relationship between maternal consumption of other foods during lactation and risk of allergic rhinitis in the child.

\section{Assessment of the evidence ${ }^{\text {viii }}$}

\section{Egg}

The following conclusion statement was supported by two RCTs ${ }^{7,14}$ and one cohort study ${ }^{4}$ and was graded moderate. Below, the individual grading elements are discussed separately for every study design.

"Moderate evidence indicates that lower or restricted consumption of eggs during pregnancy does not reduce the risk of allergic rhinitis in the child."

As outlined and described below, the body of evidence examining consumption of eggs during pregnancy and risk of allergic rhinitis in the child was assessed for the following elements used when grading the strength of evidence.

- Risk of bias was graded as moderate for the RCTs (Table 6) and limited for the cohort studies (Table 8).

- RCTs: The two RCTs included in this body of evidence had notable flaws, as described below:

- Falth-Magnusson et $\mathrm{al}^{7}$ reported issues with adherence to the assigned intervention. There was differential adherence to the intervention: 79 of the 104 mothers randomized to the intervention group adhered to the cow milk- and egg-free diet and provided follow-up data on their children at 18 months, while 102 of the 108 women randomized to control group adhered to the standard diet and provided follow-up data on their children at 18 months.

- Lilja et $\mathrm{al}^{14}$ reported potential issues with randomization with mothers in the intervention group reporting significantly higher $\operatorname{lgE}$ levels and greater prevalence of atopic eczema when compared to the control group. There were deviations from the intended intervention with some of the women in the intervention group continuing with the intervention through lactation. There were challenges with selective reporting of results. Although outcome was assessed throughout infancy and early childhood, atopic dermatitis was not reported at individual time points.

- Prospective cohort study: Similar to the RCTs, the cohort study ${ }^{4}$ had serious flaws in the design and conduct of the study:

- At least one of the key confounders was not controlled for in the cohort

\footnotetext{
viii A detailed description of the methodology used for grading the strength of the evidence is available on the NESR website: https://nesr.usda.gov/2020-dietary-guidelines-advisory-committee-systematicreviews and in Part $C$ of the following reference: Dietary Guidelines Advisory Committee. 2020.

Scientific Report of the 2020 Dietary Guidelines Advisory Committee: Advisory Report to the Secretary of Agriculture and the Secretary of Health and Human Services. U.S. Department of Agriculture, Agricultural Research Service, Washington, DC.
} 
study.

- Outcome data were self-reported and potentially subjective as they were reported by the participants who were aware of the exposure.

- The study did not have a pre-registered data analysis plan.

- Consistency was graded as strong for the RCTs and grade not assignable for the prospective cohort study.

- RCTs: There was a strong consistency in the findings. Both RCTs $\mathrm{s}^{7,14}$ showed no relationship between avoidance of egg during pregnancy and risk of allergic rhinitis in the child.

- Prospective cohort study: Consistency could not be assessed since only one prospective cohort study was included in this body of evidence.

- Directness was graded strong for the RCTs and limited for the prospective cohort study.

- RCTs: Both trials ${ }^{7,14}$ were designed to assess the relationship between maternal avoidance of egg during pregnancy and development of atopic diseases in infants and toddlers up to 18 months of age.

- Prospective cohort study: Although the study ${ }^{4}$ focused on addressing the systematic review question, this study is part of the larger Project Viva cohort that addressed associations between different exposures and outcomes.

- Precision was graded moderate for the RCTs and limited for the prospective cohort study.

- RCTs: Precision was considered moderate because both trials had a reasonable sample size (baseline $n=171^{14}$ and $212^{7}$ ). However, power calculations were not reported in either of the studies. Neither of the studies unduly influenced the findings of this systematic review and removing a single study from this body of evidence would not likely change the conclusions.

- Prospective cohort study: Precision for the prospective cohort study was considered to be limited because only one study was included in this body of evidence.

- Generalizability was graded limited for both the RCTs and the prospective cohort study.

- RCTs: Both trials were conducted in Sweden in the 1980s. However, the studies provided very minimal information on maternal age, race/ethnicity and other socioeconomic characteristics and thus applicability of the findings to the U.S. population is unclear. Given the dearth of information, generalizability for RCTs was graded limited.

- Prospective cohort study: Although the study was conducted in the U.S., the participants were predominantly White and were of mid-to-high SES. Based on the characteristics of the participants in the cohorts, it is unclear if the findings would be representative of U.S. mothers and hence generalizability was graded limited. 


\section{Dietary patterns}

The following conclusion statement was supported by three articles ${ }^{1,15,29}$ and was graded "limited." The individual grading elements are discussed below.

"Limited evidence suggests that dietary patterns during pregnancy are not associated with the risk of allergic rhinitis in the child."

As outlined and described below, the body of evidence examining dietary patterns during pregnancy and risk of allergic rhinitis in the child was assessed for the following elements used when grading the strength of evidence.

- Risk of bias (Table 8) was graded as limited for the prospective cohort studies because of the serious flaws in the design and conduct of the studies, as described below:

- At least one of the key confounders was not controlled for in all of the included studies.

- Dietary data reflected dietary intake at a single time point during pregnancy and may not have reflected intake during the entire period of pregnancy.

- Outcome data were self-reported and potentially subjective as they were reported by the participants who were aware of the exposure.

- None of the included studies had a pre-registered data analysis plan.

- Consistency was graded as strong since all of the included studies consistently showed no association between maternal DPs and risk of allergic rhinitis in the child.

- Directness was graded as limited. While the articles included in this body of evidence focused on addressing the systematic review question, the data are from larger cohort studies that address associations between different exposures and outcomes.

- Precision was considered moderate because of the adequate number of studies that were sufficiently powered to assess the association. The two U.K.-based articles $^{1,29}$ (from the same ALSPAC cohort) had a sample size of at least 1,000 participants at baseline, and the Singapore-based cohort ${ }^{15}$ had a baseline sample size of 735. No single cohort unduly affected the findings of this systematic review and removing one study would not impact the overall conclusions.

- Generalizability was graded as limited. The two articles that were from the same U.K.-based ALSPAC cohort ${ }^{1,29}$ reported that most of the participants were White and were of mid-to-high SES. The Singapore-based cohort study reported that a majority of the participants were Chinese (approximately 59 percent). None of these studies were conducted in the U.S. Based on the characteristics of the participants in the included studies, it is unclear if the findings would be representative of the U.S. mothers and hence generalizability was graded limited.

\section{Other foods}

The bodies of evidence that assessed the relationship between:

- Cow milk products (fermented or non-fermented) consumed during pregnancy, or during both pregnancy and lactation,

- Fish, peanuts, tree nuts, soybean, wheat, and foods not commonly considered 
to be allergens, such as meat, vegetables, and fruits consumed during pregnancy,

and risk of allergic rhinitis in the child were rated 'grade not assignable' for one or more of the following reasons:

- a very small body of evidence, usually less than two studies,

- heterogeneity in the findings,

- serious flaws in the design and conduct of the study, and

- limited generalizability of the findings to the U.S. population.

\section{Evidence synthesis - Asthma}

With 20 included articles, ${ }^{1-4,6,8,9,17-20,27,29-34,36,38}$ including two RCTs ${ }^{8,34,36}$ and nine prospective cohort studies, ${ }^{1-4,6,8,9,17-20,27,29-34,36,38}$ there is a substantial body of evidence available to examine the relationship between maternal diet during pregnancy and/or lactation and asthma in the child (Table 5).

\section{Cow milk products}

Pregnancy: Four prospective cohort studies ${ }^{3,4,18,32}$ assessed the association between maternal cow milk product consumption and risk of asthma. Of these, two European cohort studies showed no association between maternal cow milk product consumption (i.e. probiotic milk and yogurt, ${ }^{3}$ dairy $^{32}$ ) and asthma risk in children.

Two other studies reported a significant association, at least in certain sub-groups. Specifically, the U.S.-based cohort study ${ }^{4}$ showed that a higher consumption of milk during the first trimester was associated with a lower asthma risk at 7.9 years. However, there was no association between maternal milk intake second trimester and subsequent asthma risk in the child. The Denmark-based cohort study ${ }^{18}$ showed that dairy product and milk intake during pregnancy was not associated with childhood asthma. The association between yogurt intake and asthma risk was mixed. Higher consumption of maternal low-fat yogurt intake (vs. lower intake) during pregnancy was associated with a greater risk ( $P$ for trend=0.03) for "ever admitted" asthma (as described in the study) in the child, whereas higher maternal full-fat yogurt intake during pregnancy, compared to lower intake, was associated with a reduced risk ( $P$ for trend=0.01) for "ever prescribed asthma" (as described in the study) in the child.

Pregnancy and Lactation: One Japan-based $\mathrm{RCT}^{8}$ showed no association between maternal avoidance of cow milk products during pregnancy and lactation and risk of asthma in children. Two articles ${ }^{34,36}$ from a single U.S. trial met the inclusion criteria. The findings from these studies are presented in Table 5. However, they were not considered in evidence synthesis, grading, or when drawing conclusions for the reasons described above (p. 31).

Lactation: One Finland-based prospective cohort study ${ }^{38}$ assessed the association between maternal milk and milk product consumption during lactation (3 months postpartum) and asthma risk in the child at 5 years and reported no association.

\section{Egg}

Pregnancy: Two studies, a U.S.-based cohort study with a baseline sample size of $2,128^{4}$ and U.K.- based study with a baseline sample size of $1,715,4,32$ assessed the 
association between maternal egg consumption during pregnancy and risk of asthma. Neither of the studies showed an association between maternal egg consumption during pregnancy and risk of asthma in children.

Pregnancy and lactation: Two articles ${ }^{34,36}$ from a single U.S. trial met the inclusion criteria; however, they were not considered in evidence synthesis, grading, or when drawing conclusions for the reasons described above (p. 31).

Lactation: There were no studies that assessed the relationship between maternal egg consumption during lactation and risk of asthma in the child.

\section{Fish}

Pregnancy: Three European cohort studies (with a baseline sample size of 28,936 in the Denmark-based study, ${ }^{19} 3,963$ in the Netherlands-based study, ${ }^{32}$ and 897 in the Ireland-based study ${ }^{30}$ ) assessed the association between maternal fish consumption during pregnancy and risk of asthma in children. Two of these studies ${ }^{30,32}$ showed no association with the risk of asthma in children. In the third study, ${ }^{19}$ fish intake during pregnancy was not associated with the risk of "ever admitted" asthma (as described in the study) or current asthma at 7 years. However, no fish intake (vs. high fish intake) during pregnancy was associated with a higher risk of "ever prescribed asthma" (as described in the study) in the child.

Lactation: One Finland-based prospective cohort study ${ }^{38}$ assessed the association between maternal fish consumption during lactation ( 3 months postpartum) and asthma risk in the child at 5 years and reported no association.

\section{Peanut, tree nut and seed}

Pregnancy: Three prospective cohort studies assessed the association between peanut/nut consumption during pregnancy and risk of asthma in the child. The evidence was mixed. Specifically, the Danish cohort study ${ }^{17}$ reported that higher peanut (and pistachio) consumption, compared to lower consumption, was associated with a reduced risk ( $P$ for trend $=0.002$ ) of "ever admitted" asthma (as described by the study). The Netherlands-based study, ${ }^{32}$ however, showed that higher nut product (including peanut butter) consumption was associated with a higher risk of childhood asthma at 3-8 years, when compared to lower intake. In the U.S.-based study ${ }^{4}$, peanut consumption during the first or second trimesters was not associated with the risk of asthma in the child.

The Denmark-based cohort study ${ }^{17}$ also assessed the association between maternal tree nut consumption during pregnancy and asthma. Higher tree nut consumption, compared to lower consumption, was associated with a reduced risk ( $P$ for trend $=0.0003$ ) of "ever prescribed" asthma (as described by the study) in the child. However, the Netherlands-based study 32 noted that consumption of nuts (including tree nuts, peanuts, almonds, Brazil nuts, cashews, macadamia nuts, pistachios) was not associated with the risk of asthma in children.

There were no studies that showed an association between maternal seed consumption during pregnancy and risk of asthma in the child.

Pregnancy and lactation: Two articles ${ }^{34,36}$ from a single U.S. trial met the inclusion criteria for asthma; however, they were not considered in evidence synthesis, grading, or when drawing conclusions for the reasons described above (p. 31).

Lactation: None of the studies assessed an association between maternal peanut, tree 
nut, or seed consumption during lactation and risk of asthma in the child.

Soy

Pregnancy: The only prospective cohort study ${ }^{4}$ that assessed the relationship between maternal soybean consumption during pregnancy and risk of asthma in childhood showed no association.

Pregnancy and lactation: Two articles ${ }^{34,36}$ from a single U.S. trial met the inclusion criteria for asthma; however, they were not considered in evidence synthesis, grading, or when drawing conclusions for the reasons described above (p. 31).

Lactation: No other studies assessed the relationship between maternal soybean consumption during lactation and risk of asthma in the child.

\section{Dietary Patterns}

Pregnancy: Four cohort studies ${ }^{1,6,9,27,29}$ that assessed an association between maternal DPs and risk of asthma in the child. As noted below, studies assessed a variety of DPs (as described below):

1. Healthy Eating Index $(\mathrm{HEI})^{6}$

2. Dietary Inflammatory Index (DII) $)^{6,9}$

3. Diet quality score ${ }^{27}$

4. Mediterranean diet score ${ }^{1}$

5. Health conscious DP, Traditional DP, Processed DP, Vegetarian DP and Confectionary DP29

Despite the heterogeneity in DPs, four articles that assessed the maternal DPs during pregnancy showed no association with the risk of asthma in the child. The only exception was the Irish study ${ }^{6}$ that showed that lower diet quality, assessed using HEI and DII, was associated with a higher risk of asthma.

Lactation: There were no studies that assessed the relationship between maternal DPs during lactation and asthma risk in the child.

\section{Other foods}

Pregnancy: Seven articles assessed the relationship between maternal consumption of other foods such as vegetables, ${ }^{2,5,23,30-32}$ fruits, ${ }^{2,31,32}$ wheat/whole grains, ${ }^{4,31}$ and asthma risk and noted the following:

- Vegetables: Of the four prospective cohort studies ${ }^{2,30-32}$ that assessed the association between maternal vegetable consumption during pregnancy and risk of asthma, none showed an association with asthma risk. All four studies were conducted in Europe and had a baseline sample size of at least 1,000 participants (except one study ${ }^{30}$ with a baseline sample size of 897 ).

- Fruits: Three prospective cohort studies ${ }^{2,30-32}$ assessed the association between maternal fruit consumption during pregnancy and risk of asthma in children. Specifically, the U.K.-based cohort study reported no association between maternal total fruit consumption during pregnancy and risk of asthma at 5 years of age. ${ }^{31}$ However, consumption of apples during pregnancy was associated with a lower risk of asthma in the child. In the second study, maternal fruit consumption was not associated with overall asthma risk from 3-8 years of age. ${ }^{32}$ However, the study noted one significant association between maternal fruit consumption and steroid 
use at 6 years of age ${ }^{32}$ Another U.K.-based study showed no association between total fruit intake and risk of asthma in the child at 7.5 years. $^{2}$

- Wheat/whole grain: Two prospective cohort studies assessed the association between maternal wheat/whole grain consumption and asthma risk in the child and neither of the studies showed an association. ${ }^{4,31}$ (Note: Whole grain included whole grains, wholemeal bread, bran flakes/sultana bran/all bran, shredded wheat/Weetabix, muesli, porridge, and brown rice).

- Other beverages: Two prospective cohort studies assessed the association between maternal beverage consumption during pregnancy and risk of asthma. Specifically, the U.S.-based study ${ }^{33}$ noted that a higher maternal consumption of sugar-sweetened beverages averaged across the first and second trimesters of pregnancy was associated with an increased odds of asthma in the child at 7.7 years, compared to low consumption. The Danish cohort study noted that higher maternal consumption of artificially-sweetened carbonated $(P$ for trend $=0.01)$ and non-carbonated soft drink intake $(P$ for trend $=0.001)$ during pregnancy was associated with a higher risk of "ever prescribed" asthma in childhood (as described in the study), compared with lower intake. However, sugar-sweetened soft drink consumption during pregnancy was not associated with the risk of childhood asthma.

- Margarine/low fat spread: One study that assessed the relationship between maternal consumption of margarine/low fat spread during pregnancy and risk of asthma in the child found no association. ${ }^{31}$

Lactation: Margarine/oils/industrial fats/butter and butter-spreads/meat and meat products: One Finnish prospective cohort study ${ }^{38}$ assessed the association between maternal consumption of margarine, oils, industrial fat (as reported in the study), meat and meat products during lactation and asthma risk in the child. The study reported that higher maternal margarine consumption at 3 months postpartum, compared to no intake, was associated with higher risk of asthma at 5 years. However, there was no association between maternal consumption of oils, industrial fats, butter and butterspreads, meat and meat products and asthma risk in the child.

\section{Assessment of the evidence ${ }^{\mathrm{ix}}$}

\section{Cow milk products}

The following conclusion statement was supported by four prospective cohort studies $^{3,4,18,32}$ and was graded "limited." The individual grading elements are discussed below.

"Limited evidence suggests that a lower consumption of cow milk products during pregnancy does not reduce risk of asthma in the child."

As outlined and described below, the body of evidence examining consumption of cow milk products during pregnancy and risk of asthma in the child was assessed for the

\footnotetext{
ix A detailed description of the methodology used for grading the strength of the evidence is available on the NESR website: https://nesr.usda.gov/2020-dietary-guidelines-advisory-committee-systematicreviews and in Part $\mathrm{C}$ of the following reference: Dietary Guidelines Advisory Committee. 2020. Scientific Report of the 2020 Dietary Guidelines Advisory Committee: Advisory Report to the Secretary of Agriculture and the Secretary of Health and Human Services. U.S. Department of Agriculture, Agricultural Research Service, Washington, DC.
} 
following elements used when grading the strength of evidence.

- Risk of bias (Table 8) was graded as limited.

- The cohort studies ${ }^{3,4,18,32}$ had serious flaws in the design and conduct of the study:

- At least one of the key confounders was not controlled for in all of the cohort studies.

- Two studies ${ }^{18,32}$ in this body of evidence had possible selection bias issues.

- Studies included in this body of evidence had co-exposures that may have been related to both the exposure and the outcome.

- Outcome data were self-reported and potentially subjective as they were reported by the participants who were aware of the exposure.

- Studies did not have a pre-registered data analysis plan. There was a high likelihood of selective reporting of the analysis in at least one study. ${ }^{18}$

- Consistency was graded as moderate.

- Two prospective cohort studies ${ }^{3,32}$ reported no association between maternal cow milk product consumption during pregnancy and risk of asthma. The third study ${ }^{4}$ reported an association between maternal milk consumption during the first trimester only (and not the second trimester) and asthma risk. The fourth study ${ }^{18}$ reported mixed findings, with higher consumption of low-fat yogurt associated with a greater risk of asthma and higher consumption of high-fat yogurt associated with lower risk of asthma. The inconsistency in findings can be partially attributed to the methodological issues, as described in the risk of bias section.

- Directness was graded as limited.

- Although the prospective cohort studies $3,4,18,32$ focused on addressing the systematic review question, these studies were part of larger cohort studies that addressed associations between different exposures/outcomes.

- Precision was graded as limited.

- Precision for the prospective cohort studies was considered to be limited. There were four prospective cohort studies that were sufficiently powered to assess the association. Baseline sample sizes included at least 1,000 participants in all these cohorts. No single cohort unduly affected the findings of this systematic review and removing one study would not impact the overall conclusions.

- Generalizability was graded as limited.

- One study ${ }^{4}$ was conducted in the U.S. and participants were predominantly White and from mid-to-high SES. The rest of the studies ${ }^{3,18,32}$ included in this body of evidence were conducted in Europe. While the studies did not report on race/ethnicity, the three European studies noted that the majority of the participants had some college education. Based on the characteristics of the participants in these cohorts, it is unclear if the findings would be representative of the U.S. mothers and hence generalizability was graded limited. 


\section{Egg}

The following conclusion statement was supported by two prospective cohort studies ${ }^{4,32}$ and was graded "limited." The individual grading elements are discussed below.

"Limited evidence suggests no relationship between eggs consumed during pregnancy and risk of asthma in the child."

As outlined and described below, the body of evidence examining consumption of eggs during pregnancy and risk of asthma in the child was assessed for the following elements used when grading the strength of evidence.

- Risk of bias (Table 8) was graded as limited for cohort studies. Both studies ${ }^{4,32}$ had serious flaws in the design and conduct of the study:

- At least one of the key confounders was not controlled for in both studies.

- The Netherlands study ${ }^{32}$ had possible selection bias issues.

- There were issues with missing data in both of the studies.

- Outcome data were self-reported and potentially subjective as they were reported by the participants who were aware of the exposure.

- Studies did not have a pre-registered data analysis plan.

- Consistency was graded as strong as both of the included studies consistently showed no association between maternal egg consumption during pregnancy and risk of asthma in the child.

- Directness was graded as limited. While the articles included in this body of evidence focused on addressing the systematic review question, the data for these articles are from larger cohort studies that addressed associations between different exposures/outcomes.

- Precision was limited. Although both studies had a sample size of at least 1,000 participants at baseline, there were only two studies included in this body of evidence. Neither study unduly affected the findings of this systematic review; removing one study would not impact the overall conclusions.

- Generalizability was graded as limited. One study ${ }^{4}$ was conducted in the U.S. with predominantly White participants who were of mid-to-high SES. The Dutch study ${ }^{32}$ noted that approximately 22 percent had low maternal education, although the study did not define "low." There was no information on the race/ethnicity of the participants. Based on the characteristics of the participants in the included studies, it is unclear if the findings would be representative of U.S. mothers and hence generalizability was graded limited.

\section{Fish}

The following conclusion statement was supported by three prospective cohort studies $^{19,30,32}$ and was graded "limited." The individual grading elements are discussed below.

"Limited evidence suggests no relationship between fish consumed during pregnancy and risk of asthma in the child." 
As outlined and described below, the body of evidence examining consumption of fish during pregnancy and risk of asthma in the child was assessed for the following elements used when grading the strength of evidence.

- Risk of bias (Table 8) was graded limited for the prospective cohort studies because of the serious flaws in the design and conduct of the studies, as described below:

- At least one of the key confounders was not controlled for in all of the cohort studies.

- All three studies in this body evidence had possible selection bias issues.

- Studies included in this body of evidence had co-exposures that may have been related to both exposure and outcome.

- Outcome data were self-reported and potentially subjective as they were reported by the participants who were aware of the exposure.

- Studies did not report a pre-registered data analysis plan. There was a possibility of selective reporting of the analysis in at least one of the three studies. ${ }^{30}$

- Consistency was graded as moderate as two of the included studies consistently showed no association between maternal fish consumption during pregnancy ${ }^{30,32}$ and risk of asthma. In the third study, fish intake during pregnancy was not associated with risk of "ever admitted" asthma or current asthma at 7 years. ${ }^{19}$ However, no fish intake (vs. high fish intake) during pregnancy was associated with a higher risk of "ever prescribed" asthma.

- Directness was graded as limited. While the articles included in this body of evidence focused on addressing the systematic review question, the data for these articles are from larger cohort studies that addressed associations between different exposures/outcomes.

- Precision was considered moderate because there was an adequate number of studies that was sufficiently powered to detect the association. The sample sizes in all three studies were reasonable and ranged from $897^{30}$ to 28,936 participants ${ }^{19}$ at baseline. No single cohort unduly affected the overall findings of this systematic review and removing one study would not impact the overall conclusions.

- Generalizability was graded as limited. None of these studies were conducted in the U.S. These European cohort studies did not report the race/ethnicity of the participants; however, the Viljoen et $\mathrm{al}^{30}$ reported that all participants were 100 percent Ireland-born. Based on the characteristics of the participants in the included studies, it is unclear if the findings would be representative of U.S. mothers and hence generalizability was graded limited.

\section{Other foods}

The bodies of evidence that assessed the relationship between:

- Cow milk products consumed during both pregnancy and lactation, or lactation,

- Maternal dietary patterns or peanuts, tree nuts, soybean, and other foods such as wheat/whole grains, vegetables, fruits, beverages, and margarine consumed 
during pregnancy,

- Fish, and other foods, such as margarine, oil, butter and butter-spreads, industrial fat, meat, and meat products consumed during lactation,

and risk of asthma in the child were rated 'grade not assignable' for one or more of the following reasons:

- a very small body of evidence, usually two studies or less,

- heterogeneity in the findings,

- serious flaws in the design and conduct of the study, and

- limited generalizability of the findings to the U.S. population.

Publication bias is an important consideration in this systematic review and can be perceived to be a concern because of the fewer number of studies within each intervention/exposure-outcome relationships. However, this systematic review included studies that reported null findings in both relatively small and large studies, which suggests that publication bias may be less of a concern in this body of evidence. 


\section{Research recommendations}

- Include well-designed RCTs and prospective cohort studies that assess the relationship between maternal diet and risk of atopic dermatitis/eczema, food allergies, allergic rhinitis and asthma.

- Assess intervention/exposure at multiple time points during pregnancy and/or during lactation.

- Adjust for key confounding factors in observational studies, including maternal age, race/ethnicity, socioeconomic status, smoking, family history of atopic allergic diseases, gestational age at birth, birth weight, mode of delivery, human milk feeding practices (intensity, duration), types of complementary foods and beverages and timing of its introduction, urban/rural environment, animals/pets/farming exposure; sex, maternal substance use (alcohol, drug use), and Indoor and outdoor environment.

- Include diverse populations with varying age groups and different racial/ethnic and socioeconomic backgrounds. 


\section{Included articles}

1. Bedard A, Northstone K, Henderson AJ, Shaheen SO. Mediterranean diet during pregnancy and childhood respiratory and atopic outcomes: birth cohort study. Eur Respir J. 2020;55(3). doi:10.1183/13993003.01215-2019

2. Bedard A, Northstone K, Holloway JW, Henderson AJ, Shaheen SO. Maternal dietary antioxidant intake in pregnancy and childhood respiratory and atopic outcomes: birth cohort study. Eur Respir J. 2018;52(2). doi:10.1183/13993003.00507-2018

3. Bertelsen RJ, Brantsaeter AL, Magnus MC, et al. Probiotic milk consumption in pregnancy and infancy and subsequent childhood allergic diseases. J Allergy Clin Immunol. 2014;133(1):165-171 e161-168. doi:10.1016/j.jaci.2013.07.032

4. Bunyavanich S, Rifas-Shiman SL, Platts-Mills TA, et al. Peanut, milk, and wheat intake during pregnancy is associated with reduced allergy and asthma in children. J Allergy Clin Immunol. 2014;133(5):1373-1382. doi:10.1016/j.jaci.2013.11.040

5. Chatzi L, Garcia R, Roumeliotaki T, et al. Mediterranean diet adherence during pregnancy and risk of wheeze and eczema in the first year of life: INMA (Spain) and RHEA (Greece) mother-child cohort studies. Br J Nutr. 2013;110(11):20582068. doi:10.1017/S0007114513001426

6. Chen LW, Lyons B, Navarro P, et al. Maternal dietary inflammatory potential and quality are associated with offspring asthma risk over 10-year follow-up: the Lifeways Cross-Generation Cohort Study. Am J Clin Nutr. 2020;111(2):440-447. doi:10.1093/ajcn/nqz297

7. Falth-Magnusson K, Kjellman NI. Development of atopic disease in babies whose mothers were receiving exclusion diet during pregnancy--a randomized study. J Allergy Clin Immunol. 1987;80(6):868-875. doi:10.1016/s00916749(87)80279-8

8. Fukushima Y, Iwamoto K, Takeuchi-Nakashima A, et al. Preventive effect of whey hydrolysate formulas for mothers and infants against allergy development in infants for the first 2 years. J Nutr Sci Vitaminol (Tokyo). 1997;43(3):397-411. doi:10.3177/jnsv.43.397

9. Hanson C, Rifas-Shiman SL, Shivappa N, et al. Associations of Prenatal Dietary Inflammatory Potential with Childhood Respiratory Outcomes in Project Viva. J Allergy Clin Immunol Pract. 2020;8(3):945-952 e944. doi:10.1016/j.jaip.2019.10.010

10. Herrmann ME, Dannemann A, Gruters A, et al. Prospective study of the atopy preventive effect of maternal avoidance of milk and eggs during pregnancy and lactation. Eur J Pediatr. 1996;155(9):770-774. doi:10.1007/bf02002904

11. Jedrychowski W, Perera F, Maugeri $U$, et al. Effects of prenatal and perinatal exposure to fine air pollutants and maternal fish consumption on the occurrence of infantile eczema. Int Arch Allergy Immunol. 2011;155(3):275-281. doi:10.1159/000320376

12. Lack G, Fox D, Northstone K, Golding J, Avon Longitudinal Study of P, Children Study $\mathrm{T}$. Factors associated with the development of peanut allergy in childhood. N Engl J Med. 2003;348(11):977-985. doi:10.1056/NEJMoa013536

13. Leermakers ET, Sonnenschein-van der Voort AM, Heppe DH, et al. Maternal fish consumption during pregnancy and risks of wheezing and eczema in childhood: the Generation R Study. Eur J Clin Nutr. 2013;67(4):353-359. doi:10.1038/ejcn.2013.36 
14. Lilja G, Dannaeus A, Foucard T, Graff-Lonnevig V, Johansson SG, Oman H. Effects of maternal diet during late pregnancy and lactation on the development of atopic diseases in infants up to 18 months of age--in-vivo results. Clin Exp Allergy. 1989;19(4):473-479. doi:10.1111/j.1365-2222.1989.tb02416.x

15. Loo EXL, Ong L, Goh A, et al. Effect of Maternal Dietary Patterns during Pregnancy on Self-Reported Allergic Diseases in the First 3 Years of Life: Results from the GUSTO Study. Int Arch Allergy Immunol. 2017;173(2):105113. doi:10.1159/000475497

16. Lovegrove JA, Hampton SM, Morgan JB. The immunological and long-term atopic outcome of infants born to women following a milk-free diet during late pregnancy and lactation: a pilot study. Br J Nutr. 1994;71(2):223-238. doi:10.1079/bjn19940129

17. Maslova E, Granstrom C, Hansen S, et al. Peanut and tree nut consumption during pregnancy and allergic disease in children-should mothers decrease their intake? Longitudinal evidence from the Danish National Birth Cohort. $J$ Allergy Clin Immunol. 2012;130(3):724-732. doi:10.1016/j.jaci.2012.05.014

18. Maslova E, Halldorsson TI, Strom M, Olsen SF. Low-fat yoghurt intake in pregnancy associated with increased child asthma and allergic rhinitis risk: a prospective cohort study. J Nutr Sci. 2012;1. doi:10.1017/jns.2012.5

19. Maslova E, Strom M, Oken E, et al. Fish intake during pregnancy and the risk of child asthma and allergic rhinitis - longitudinal evidence from the Danish National Birth Cohort. Br J Nutr. 2013;110(7):1313-1325. doi:10.1017/S000711451300038X

20. Maslova E, Strom M, Olsen SF, Halldorsson TI. Consumption of artificiallysweetened soft drinks in pregnancy and risk of child asthma and allergic rhinitis. PLoS One. 2013;8(2):e57261. doi:10.1371/journal.pone.0057261

21. Miyake Y, Okubo H, Sasaki S, Tanaka K, Hirota Y. Maternal dietary patterns during pregnancy and risk of wheeze and eczema in Japanese infants aged 1624 months: the Osaka Maternal and Child Health Study. Pediatr Allergy Immunol. 2011;22(7):734-741. doi:10.1111/j.1399-3038.2011.01176.x

22. Miyake Y, Sasaki S, Tanaka K, Hirota Y. Dairy food, calcium and vitamin D intake in pregnancy, and wheeze and eczema in infants. Eur Respir J. 2010;35(6):1228-1234. doi:10.1183/09031936.00100609

23. Miyake Y, Sasaki S, Tanaka K, Hirota Y. Consumption of vegetables, fruit, and antioxidants during pregnancy and wheeze and eczema in infants. Allergy. 2010;65(6):758-765. doi:10.1111/j.1398-9995.2009.02267.x

24. Miyake Y, Sasaki S, Tanaka K, Ohfuji S, Hirota Y. Maternal fat consumption during pregnancy and risk of wheeze and eczema in Japanese infants aged 1624 months: the Osaka Maternal and Child Health Study. Thorax. 2009;64(9):815-821. doi:10.1136/thx.2009.115931

25. Miyake Y, Tanaka K, Okubo H, Sasaki S, Arakawa M. Maternal fat intake during pregnancy and wheeze and eczema in Japanese infants: the Kyushu Okinawa Maternal and Child Health Study. Ann Epidemiol. 2013;23(11):674-680. doi:10.1016/j.annepidem.2013.08.004

26. Miyake Y, Tanaka K, Okubo H, Sasaki S, Arakawa M. Maternal consumption of dairy products, calcium, and vitamin $D$ during pregnancy and infantile allergic disorders. Ann Allergy Asthma Immunol. 2014;113(1):82-87. doi:10.1016/j.anai.2014.04.023 
27. Nguyen AN, Elbert NJ, Pasmans S, et al. Diet Quality throughout early life in relation to allergic sensitization and atopic diseases in childhood. Nutrients. 2017;9(8). doi:10.3390/nu9080841

28. Saito K, Yokoyama T, Miyake Y, et al. Maternal meat and fat consumption during pregnancy and suspected atopic eczema in Japanese infants aged 3-4 months: the Osaka Maternal and Child Health Study. Pediatr Allergy Immunol. 2010;21(1 Pt 1):38-46. doi:10.1111/j.1399-3038.2009.00897.x

29. Shaheen SO, Northstone K, Newson RB, Emmett PM, Sherriff A, Henderson AJ. Dietary patterns in pregnancy and respiratory and atopic outcomes in childhood. Thorax. 2009;64(5):411-417. doi:10.1136/thx.2008.104703

30. Viljoen K, Segurado R, O'Brien J, et al. Pregnancy diet and offspring asthma risk over a 10-year period: the Lifeways Cross Generation Cohort Study, Ireland. BMJ Open. 2018;8(2):e017013. doi:10.1136/bmjopen-2017-017013

31. Willers SM, Devereux G, Craig LC, et al. Maternal food consumption during pregnancy and asthma, respiratory and atopic symptoms in 5-year-old children. Thorax. 2007;62(9):773-779. doi:10.1136/thx.2006.074187

32. Willers SM, Wijga AH, Brunekreef $\mathrm{B}$, et al. Maternal food consumption during pregnancy and the longitudinal development of childhood asthma. Am J Respir Crit Care Med. 2008;178(2):124-131. doi:10.1164/rccm.200710-1544OC

33. Wright LS, Rifas-Shiman SL, Oken E, Litonjua AA, Gold DR. Prenatal and Early Life Fructose, Fructose-Containing Beverages, and Midchildhood Asthma. Ann Am Thorac Soc. 2018;15(2):217-224. doi:10.1513/AnnalsATS.201707-530OC

34.Zeiger RS, Heller S. The development and prediction of atopy in high-risk children: follow-up at age seven years in a prospective randomized study of combined maternal and infant food allergen avoidance. J Allergy Clin Immunol. 1995;95(6):1179-1190. doi:10.1016/s0091-6749(95)70074-9

35. Zeiger RS, Heller S, Mellon MH, et al. Effect of combined maternal and infant food-allergen avoidance on development of atopy in early infancy: a randomized study. J Allergy Clin Immunol. 1989;84(1):72-89. doi:10.1016/00916749(89)90181-4

36.Zeiger RS, Heller S, Mellon MH, Halsey JF, Hamburger RN, Sampson HA. Genetic and environmental factors affecting the development of atopy through age 4 in children of atopic parents: a prospective randomized study of food allergen avoidance. Pediatr Allergy Immunol. 1992. doi:10.1111/j.13993038.1992.tb00035.x

37. Jirapinyo P, Densupsoontorn N, Kangwanpornsiri C, Limlikhit T. Lower prevalence of atopic dermatitis in breast-fed infants whose allergic mothers restrict dairy products. J Med Assoc Thai. 2013;96(2):192-195.

38. Lumia M, Luukkainen $\mathrm{P}$, Kaila M, et al. Maternal dietary fat and fatty acid intake during lactation and the risk of asthma in the offspring. Acta Paediatr. 2012;101(8):e337-343. doi:10.1111/j.1651-2227.2012.02718.x 
Table 2. Description of evidence on the relationship between maternal diet during pregnancy and lactation and risk of child food allergy $x$, xi

\begin{tabular}{|c|c|c|c|}
\hline $\begin{array}{l}\text { Study and Participant } \\
\text { Characteristics }\end{array}$ & Intervention/Exposure and Outcomes & Results & $\begin{array}{l}\text { Confounding, Study Limitations, and } \\
\text { Summary of Findings }\end{array}$ \\
\hline \multicolumn{4}{|l|}{ Pregnancy } \\
\hline \multicolumn{4}{|l|}{ Prospective Cohort Studies } \\
\hline $\begin{array}{l}\text { Baseline } \mathrm{N}=2,128 \text { Analytic } \\
\mathrm{N}=616 \\
\text { Of } 1277 \text { that were followed- } \\
\text { up, } 616 \text { participants agreed } \\
\text { to have blood drawn } \\
\text { - Age: } 32.3 \text { (from other } \\
\text { Project Viva data) } \\
\text { - Race/Ethnicity: White: } 69 \% \\
\text { - SES: } \\
\text { - Maternal Education: } \\
\geq \text { college graduate: } 69.3 \% \\
\text { - Household income } \geq \$ 70 \mathrm{~K} \text { : } \\
63.0 \% \\
\text { - Reported but not tested by } \\
\text { exposure: Family history }\end{array}$ & $\begin{array}{l}\text { Food(s) or Food Group(s): } \\
\text { Maternal consumption of major food } \\
\text { allergens (servings/d } z \text {-scores) } \\
\text { - Peanut: } \\
\circ 1^{\text {st }} \text { trimester: } 0.34 \pm 0.44 \\
\circ 2^{\text {nd }} \text { trimester: } 0.36 \pm 0.43 \\
\text { - Milk: } \\
\circ 1^{\text {st }} \text { trimester: } 1.16 \pm 1.04 \\
\circ 2^{\text {nd }} \text { trimester: } 1.50 \pm 1.82 \\
\text { - Wheat: } \\
\circ 1^{\text {st }} \text { trimester: } 2.65 \pm 1.48 \\
\circ 2^{\text {nd }} \text { trimester: } 2.69 \pm 1.44 \\
\text { - Egg: } \\
\circ 1^{\text {st }} \text { trimester: } 0.32 \pm 0.30 \\
\circ 2^{\text {nd }} \text { trimester: } 0.33 \pm 0.30 \\
\text { - Soy: } \\
\circ 1^{\text {st }} \text { trimester: } 0.08 \pm 0.27 \\
\circ 2^{\text {nd }} \text { trimester: } 0.08 \pm 0.28 \\
\text { at } 10 \text { wk and } 26-28 w k \text { gestation } \\
\text { Dietary assessment methods: }\end{array}$ & $\begin{array}{l}\text { Non-significant: } \\
\text { Maternal 1st trimester intake of any } \\
\text { food allergens (milk, egg, wheat, } \\
\text { soy), P=NS } \\
\text { Maternal 2nd trimester intake of any } \\
\text { food allergens (milk, egg, wheat, } \\
\text { peanuts, or soy) P=NS } \\
\text { Stratification by parental atopy } \\
\text { showed no association between } \\
\text { intake of milk, wheat, egg, and soy } \\
\text { during 1st or 2nd trimester and food } \\
\text { allergies. }\end{array}$ & $\begin{array}{l}\text { OFCs accounted for: } \\
\text { Child sex } \\
\text { Limitations: } \\
\text { - Critical co-exposures NR } \\
\text { - Mothers who participated in the study were } \\
\text { different than those who were lost to follow- } \\
\text { up on the following characteristics: maternal } \\
\text { race, college education, income, parental } \\
\text { atopy } \\
\text { - Proportions of and reasons for missingness } \\
\text { NR by exposure } \\
\text { - Multiple exposure outcome comparisons } \\
\text { were assessed without using an appropriate } \\
\text { p-value correction } \\
\text { - Self-reported exposure and outcome (for } \\
\text { clinical symptoms) }\end{array}$ \\
\hline
\end{tabular}

\pm indicates values of Mean \pm SD unless otherwise noted

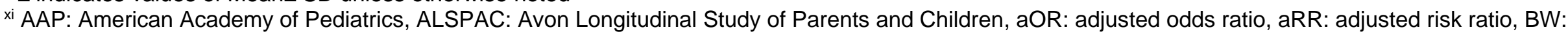
birth weight, CD: Cow's milk diet, CFB: complementary food and beverage, Cl: confidence interval, d: day, FFQ: food frequency questionnaire, Gl: gastrointestinal, HMF: human milk feeding, MD: Mom hypoallergenic formula, mo: month(s), NR: not reported, NS: non-significant, OFCs: other factors

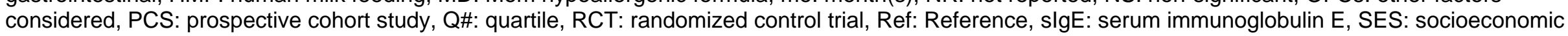
status; wk: week(s), y: year(s) 
Study and Participant

Characteristics

\section{Intervention/Exposure and Outcomes Results}

Maternal dietary assessments at the

first and second trimester visits were based on a validated 166 -item semiquantitative FFQ modified for pregnancy. The total servings per day of each major food allergen (peanut,

milk, wheat, egg, and soy) were calculated by summing the servings per day of the foods on the FFQ containing these respective food allergens.

\section{Outcome:}

Food Allergy at $~ 7.9 \mathrm{y}$

\section{Outcome assessment methods:}

Sensitization to a food allergen was considered positive if the respective allergen slgE level was $0.35 \mathrm{kU} / \mathrm{L}$ or more. Prescription of an epinephrine autoinjector was assessed with the question, "Has a health care professional, such as a doctor, physician assistant, or nurse

practitioner, ever prescribed an EpiPen for your child?" A child was considered

to have food allergy to peanut, milk, wheat, egg, and/or soy if he or she had a slgE level of $0.35 \mathrm{kU} / \mathrm{L}$ or more to the particular food and EpiPen prescribed.

\section{Lack, 2003 ${ }^{12}$; U.K.} PCS, ALSPAC

\section{Food(s) or Food Group(s):}

Soybean meat consumption (yes/no)

Baseline $\mathrm{N}=13,971$ Analytic Assessed once at 32 wk gestation $\mathrm{N}=11,352$
Confounding, Study Limitations, and Summary of Findings

- Pre-registered data analysis plan NR

\section{Summary:}

Maternal intake of any major food allergens (peanuts, milk, egg, wheat or soy) in the 1st trimester or 2nd trimester was not associated with risk of food allergy in the child at $7.9 \mathrm{y}$.

\section{Significant:}

Non-significant:

Soybean meat consumption
Key confounders accounted for:

None

OFCs accounted for: 
Study and Participant Characteristics

- Age: NR

- Race/Ethnicity: NR

- SES: NR

- Baseline characteristics

NR by exposure

\section{Intervention/Exposure and Outcomes Results}

Yes vs No (Ref): $P=N S$

Outcome: Food allergy at $38 \mathrm{mo}$

\section{Outcome assessment methods:}

Children $\leq 38$ mo who had peanut allergy on the basis of responses to questions about food avoidance and reactions to particular foods were identified. Affected children were also identified from responses to questions on the questionnaire regarding previous hospitalizations and clinical investigations. Forty-nine mothers of children who had a reaction to peanuts according to questionnaire responses were interviewed in detail over the telephone about the reaction. Children who were found to have had a reaction to peanuts (age range, four to six years) underwent skin testing and doubleblind, placebo-controlled food challenge. Skin testing was performed with peanut (concentration, 1:20 [wt/vol] in 50 percent glycerol). A skin test was considered positive if it resulted in a palpable wheal at least $3 \mathrm{~mm}$ in diameter. Peanut food allergy at 4-6y confirmed by double-blind, placebocontrolled food challenge $\mathrm{P}=\mathrm{NS}$
Only among those with positive peanut challenge: Yes vs No (Ref):

\section{Pregnancy and Lactation \\ Randomized Controlled Trials}

Fukushima, $1997^{8}$; Japan Food(s) or Food Group(s): RCT

\section{Significant:}

Note, CD had significantly higher odds of overall allergies vs MD at
Confounding, Study Limitations, and Summary of Findings

None

\section{Limitations:}

- No key confounders controlled for

- Exposure status and methods used to assess the exposure are not well defined. It is not

clear what soybean meat included.

- Potential for selection bias, since the participants that only responded to the allergy question in the questionnaire were included in the study

- No information on deviation from intended exposure or on proportions and reasons for missing data across exposure groups

- No pre-registered data analysis plan

\section{Summary:}

Maternal soybean meat consumption during pregnancy was not associated with risk of peanut allergy at 4-6 y. 


\begin{tabular}{|c|c|c|c|}
\hline $\begin{array}{l}\text { Study and Participant } \\
\text { Characteristics }\end{array}$ & Intervention/Exposure and Outcomes & Results & $\begin{array}{l}\text { Confounding, Study Limitations, and } \\
\text { Summary of Findings }\end{array}$ \\
\hline $\begin{array}{l}\text { Baseline } \mathrm{N}=350 \text { Analytic } \\
\text { N=283 } \\
\text { - Age: NR } \\
\text { - Race/Ethnicity: 100\% from } \\
\text { Japan } \\
\text { - SES: NR } \\
\text { - Significant differences by } \\
\text { exposure (by design): } \\
\text { HMF, CFB } \\
\text { - No differences by } \\
\text { exposure: Smoking, Family } \\
\text { history, BW, Pets } \\
\text { - 76\% had a positive } \\
\text { history of allergy (i.e., both } \\
\text { parents or one parent or } \\
\text { sibling only had history of } \\
\text { allergies) }\end{array}$ & $\begin{array}{l}\text { - CD: Mothers instructed to consume } \\
\text { >200mL/d cow milk, } n=140 \\
\text { randomized, } n=127 \text { analyzed } \\
\text { - MD: Mothers instructed to consume } \\
\text { >200mL/d a casein-free, } \\
\text { hypoallergenic formula and to avoid } \\
\text { cow milk and reduce consumption of } \\
\text { cow milk products, n=140 randomized, } \\
\mathrm{n}=102 \text { analyzed } \\
\text { - AF: Mothers instructed to consume } \\
\text { >200mL/d a casein-free, } \\
\text { hypoallergenic formula and to avoid } \\
\text { cow milk and reduce consumption of } \\
\text { cow milk products, } n=70 \text { randomized, } \\
\mathrm{n}=54 \text { analyzed (Not randomized, no } \\
\text { relevant comparisons included, except } \\
\text { as co-variates in logistic regressions) } \\
\text { o Also supplemented with } 1000 \text { mg/d } \\
\text { Ca } \\
\text { during late pregnancy through end of } \\
\text { lactation up to } 6 \text { mo postpartum } \\
\text { From birth to } 6 \text { mo, the infants in the } \\
\text { MD and CD groups were exclusively } \\
\text { HMF or mixed-fed with breast milk and } \\
\text { casein-free, hypoallergenic formula } \\
\text { when breast milk was insufficient. The } \\
\text { infants in the AF group were mixed-fed } \\
\text { with breast milk and a formula with } \\
\text { similar whey:casein ratio as breastmilk } \\
\text { for the corresponding } 6 \text { mo. Infants who } \\
\text { were fed breast milk exclusively from } \\
\text { birth to } 4 \text { mo were excluded from the AF } \\
\text { group. }\end{array}$ & $\begin{array}{l}\text { Non-significant: } \\
\text { Median total IgE levels at } 4 \mathrm{mo} \text {, } \\
\text { P=NS } \\
\text { - CD: } 3.20 \mathrm{IU} / \mathrm{mL} \\
\text { - MD: } 3.36 \mathrm{IU} / \mathrm{mL} \\
\text { Overall allergies at } 24 \mathrm{mo}, \mathrm{P}=\mathrm{NS} \\
\text { - CD: aOR=1.75, } 95 \% \mathrm{Cl} \text { : }(0.94 \text {, } \\
3.25) \\
\text { - MD: (Ref) } \\
\text { Note, odds of overall allergies NS at } \\
6 \text { and } 18 \text { mo. However, due to } \\
\text { inclusion of asthma these results do } \\
\text { not meet the NESR criteria }\end{array}$ & $\begin{array}{l}\text { OFCs accounted for: } \\
\text { None } \\
\text { Limitations: } \\
\text { - } \quad \text { Randomization process NR } \\
\text { - } \quad \text { MD still consumed cow milk products, but } \\
\text { had lower intake than CD } \\
\text { - Attrition higher in MD group because } \\
\text { women excluded from analysis if they } \\
\text { consumed cow milk } \\
\text { Proportions of missing data seem to differ, } \\
\text { reasons for missingness NR } \\
\text { Participants were aware of exposure status } \\
\text { and outcomes were self-reported } \\
\text { Pre-registered data analysis plan NR } \\
\text { Summary: } \\
\text { Consuming diets replacing cow milk with } \\
\text { casein-free hypoallergenic formula with } \\
\text { reduced consumption of cow milk products } \\
\text { from late pregnancy through lactation did not } \\
\text { impact overall allergies, including atopic } \\
\text { eczema, asthma and allergic rhinitis in the } \\
\text { child. }\end{array}$ \\
\hline & Dietary assessment methods: & & \\
\hline
\end{tabular}


Study and Participant

Characteristics

\section{Intervention/Exposure and Outcomes}

Results

Daily food diary from late pregnancy

until $6 \mathrm{mo}$ postpartum, recording the

amount of casein-free, hypoallergenic

formula, cow milk, cow milk products,

eggs, meat, and soy products

consumed.

\section{Outcome:}

Overall allergies (eczema, asthma, and/or allergic rhinitis)

at $24 \mathrm{mo}$

\section{Outcome assessment methods:}

Self-reported by questionnaire. Specific allergies defined when symptoms

chronically lasted for more than a few weeks.

Umbilical cord blood and venous blood obtained from all infants at birth and $4 \mathrm{mo}$ of age. Total $\lg \mathrm{E}$ in serum was measured using a radioimmunoassay (RIA) kit. Cow milk-specific lgE level in serum was measured by the CAPRAST method. Specific IgE (RAST) was considered positive when the level was more than $0.35 \mathrm{UA} / \mathrm{mL}$.

\section{Zeiger, $1989^{35}$; U.S.}

RCT

Baseline $\mathrm{N}=379$ Analytic $\mathrm{N}=288$

Power analysis: Yes

\section{Food(s) or Food Group(s):}

- Control: Mothers encouraged to follow standard diets during $3^{\text {rd }}$ trimester of pregnancy and lactation, $n=212$

o Cow milk-based whey infant formula provided for supplementation or weaning through 12 mo postpartum. CFB
Confounding, Study Limitations, and

Summary of Findings

\section{Significant:}

Food allergy at $12 \mathrm{mo}$

- Total cumulative prevalence, $\mathrm{P}=0.007$

- Control ( $n=177): 16.4 \%$

○ Prophylactic $(n=99): 5.0 \%$

\section{Key confounders accounted for:}

Age, Race/Ethnicity, SES, Smoking, Family history, HMF, CFB, Pets

\section{Limitations:}


Study and Participant Characteristics

Of these families, 14 in each group were found to be not atopic, which eliminated them from the study

Baseline characteristics for 288 participants

- Age: 28.6 y

- Race/Ethnicity:

o Non-White: 12.5\%

- SES:

- Maternal occupation: white collar: $~ 54.0 \%$

- Family income $<\$ 20,000 / y: ~ 9.7 \%$

- Maternal Education:

- sHigh school: $13.6 \%$

- Characteristics reported with significant differences by exposure

- BW (term, singletons)

- Characteristics reported with no differences by exposure

Smoking

- Family history

o HMF

○ CFB

Animal/Pets/Farming exposure

Families were included in the study if at least one parent met the following criteria: history of an atopic disorder and specific lgE by

\section{Intervention/Exposure and Outcomes Results}

encouraged: no solids < $4 \mathrm{mo}$,

cereal at 4 mo, followed by

vegetables, fruits and egg yolks at

$6 \mathrm{mo}$, meats at $8 \mathrm{mo}$, and whole

cow milk and egg whites at 12 mo.

- Prophylaxis: Instructed to avoid totally all milk (dairy), egg, and peanut products, avoid concentrated soy foods (i.e., tofu), $\leq 2$ servings/d wheat, with other grains to fulfill cereal and starch requirements during $3^{\text {rd }}$ trimester of pregnancy and lactation, $\mathrm{n}=167$

o In addition to prenatal vitamins, the maternal diet was supplemented with a total of $1500 \mathrm{mg} / \mathrm{d} \mathrm{Ca}$.

- A casein hydrolysate infant formula with low sensitization potential provided for supplementation or weaning through 12 mo postpartum. CFB encouraged: no solids $<6 \mathrm{mo}$, non-legume vegetables, followed by rice cereal at $7 \mathrm{mo}$, meats at $8 \mathrm{mo}$, non-citrus fruits and juices at $9 \mathrm{mo}$, and cow milk at 12 mo. Wheat, soy, corn, and citrus introduced thereafter at monthly intervals, followed by egg at 24 mo and peanuts and fish at $36 \mathrm{mo}$.

- Both groups encouraged to feed human milk for $\geq 4-6$ mo

\section{Dietary assessment methods:}

Women were randomly assigned to groups. In addition to instructions described above, women in prophylaxis group attended a dietary class was held

Confounding, Study Limitations, and Summary of Findings

Food allergy at 24 mo

- Total cumulative prevalence, $P=0.005$

o Control $(n=169): 20.1 \%$

- Prophylactic $(n=97): 7.2 \%$

- Probable cumulative prevalence, $\mathrm{P}=0.021$

o Control $(n=169): 11.2 \%$

o Prophylactic $(n=97): 3.1 \%$

Period prevalence of food-specific allergies, at each time point at 4, 12, and 24 mo

- Milk

○ $4 \mathrm{mo}, \mathrm{P}=\mathrm{NS}$

o 12 mo, Control > Prophylactic, $P=0.013$

Cumulative prevalence of foodspecific allergies, at each time point at 4,12 , and 24 mo

- Any food

० $4 \mathrm{mo}, \mathrm{P}=0.069$

० $12 \mathrm{mo}, \mathrm{P}=0.074$

$\circ 24 \mathrm{mo}$, Control > Prophylactic, $\mathrm{P}=0.019$

- Egg, $\mathrm{P}=\mathrm{NS}$

- Milk

○ $4 \mathrm{mo}, \mathrm{P}=\mathrm{NS}$

$\circ 12 \mathrm{mo}$, Control > Prophylactic, $\mathrm{P}=0.007$

○ 24mo, Control > Prophylactic, $\mathrm{P}=0.001$

\section{Non-significant:}

Food allergy at 4 mo
- Significantly more non-White fathers in prophylaxis group, and trend to more mothers with low education in control group

- 6 pairs of twins omitted from control group after randomization

- BW 2.9\% lower in term, singletons in prophylactic group ( $\mathrm{P}=0.044)$; Overall, BW similar between groups

- More women in the prophylactic-treated group withdrew before delivery because of the protocol's dietary restrictions. As a result, halfway through recruitment, randomization was changed from 40:60 (treatment: control) to 50:50 with a new computerized list of random numbers.

- Some missing data, but power calculation suggests the analytic $\mathrm{N}$ is sufficient to test hypotheses.

- Physician making diagnosis was aware of exposure status; outcomes required both lab tests and observations at multiple time points, with similar results for

- "probable" and "definite" diagnoses.

- No pre-registered data analysis plan.

\section{Summary:}

Maternal avoidance of milk (dairy), egg, and peanut products, avoidance of concentrated soy foods, and limited intake of wheat during the $3^{\text {rd }}$ trimester of pregnancy through lactation, and reduced infant food allergen exposure during CFB reduced the prevalence of some food allergies in the child through 24 mo. 


\begin{tabular}{|c|c|c|c|}
\hline $\begin{array}{l}\text { Study and Participant } \\
\text { Characteristics }\end{array}$ & Intervention/Exposure and Outcomes & Results & $\begin{array}{l}\text { Confounding, Study Limitations, and } \\
\text { Summary of Findings }\end{array}$ \\
\hline $\begin{array}{l}\text { skin or RAST testing. Serum } \\
\text { was obtained from the } \\
\text { participants for total and } \\
\text { specific IgE determinations. } \\
\text { Participant fathers were skin } \\
\text { tested to inhalant antigens at } \\
\text { the intake session. Mothers } \\
\text { were skin tested to foods } \\
\text { and inhalants } 4 \text { mo } \\
\text { postpartum. }\end{array}$ & $\begin{array}{l}\text { before the 3rd trimester by a licensed } \\
\text { dietitian to provide detailed instructions } \\
\text { on the maternal and infant diets, food } \\
\text { lists, recipes, and product sources. } \\
\text { Adherence to the dietary regimen was } \\
\text { ascertained in part by maternal self- } \\
\text { report and daily diaries. } \\
\text { For both groups: } 0.25 \mathrm{mg} / \mathrm{d} \text { Tri-Vi-Flor } \\
\text { given to infants according to their } \\
\text { pediatrician's preference. Foods } \\
\text { causing documented IgE sensitization } \\
\text { were removed from the infant's diet until } \\
\text { sensitization had waned or were } \\
\text { tolerated on double-blind challenge. } \\
\text { Parents received intensive education on } \\
\text { reducing environmental allergens and } \\
\text { tobacco smoke from their homes. } \\
\text { Outcome: Atopic dermatitis, Allergic } \\
\text { rhinitis, Food allergy } \\
\text { at } 1,4,8, \text { and } 12 \text { mo } \\
\text { Outcome assessment methods: } \\
\text { Food allergy defined as probable when } \\
\text { food-specific IgE was associated with } \\
\text { atopic dermatitis, urticaria/angioedema } \\
\text { induced at least twice by foods, and Gl } \\
\text { allergy. Formula allergy was a } \\
\text { component of food allergy in which any } \\
\text { of the above symptoms occurred after } \\
\text { ingestion of study formula with the } \\
\text { presence of concomitant milk-specific } \\
\text { lgE. A positive double-blind food } \\
\text { challenge or a severe food reaction with } \\
\text { coexisting food-specific IgE was } \\
\text { considered definite food allergy. }\end{array}$ & 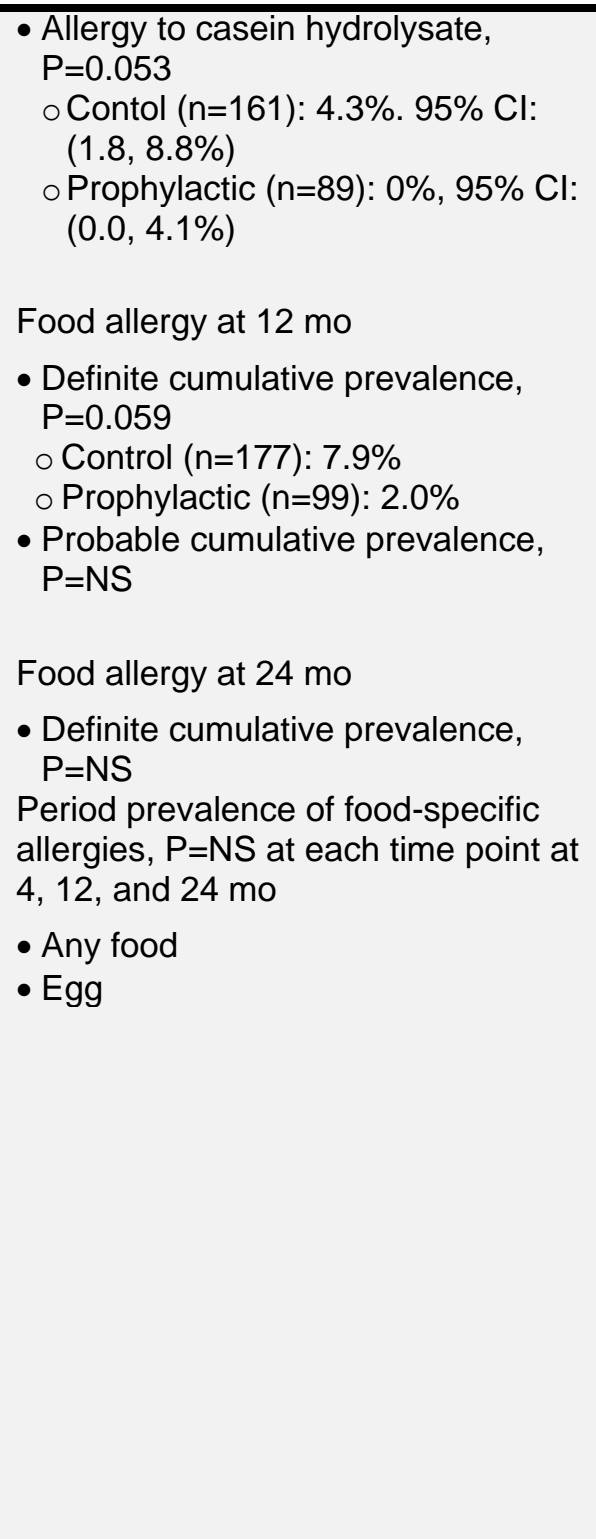 & \\
\hline
\end{tabular}


Study and Participant Characteristics

\section{Intervention/Exposure and Outcomes Results}

Confounding, Study Limitations, and Summary of Findings

Prophylactic infants used casein
hydrolysate (Nutramigen) for
supplementation or weaning, and
avoided solid foods for $6 \mathrm{mo}$; cow milk,
corn, soy, citrus, and wheat, for $12 \mathrm{mo} ;$
and egg, peanut, and fish, for 24 mo.
Infants in the control groups Enfamil, a
cow milk-based whey infant formula.
Solid foods were introduced to control-
group infants, based on AAP
recommendations.

Zeiger, 1992 ${ }^{36}$; U.S.

RCT

Baseline $\mathrm{N}=379$ Analytic $\mathrm{N}=242$ (at 3y) and $\mathrm{N}=225$ (at $4 y)$

\section{Food(s) or Food Group(s):}

- Control: Mothers encouraged to follow standard diets during 3rd trimester of

- Prophylaxis: Avoided all milk (dairy), egg, and peanut products,

concentrated soy foods (i.e., tofu), $\leq 2$ servings/d wheat during 3rd trimester of pregnancy and lactation

See Zeiger, $1989^{35}$ pregnancy and lactation

\section{Significant:}

4-year Cumulative Prevalence

Control Group vs Prophylaxis: $\mathrm{aRR}=2.5,95 \% \mathrm{Cl}=(1.1,5.6), \mathrm{P}<0.05$

\section{Non-significant:}

Period prevalence at 3 and $4 \mathrm{y}$ :

Prophylaxis vs Control: NS

- Prenatal vitamins plus

supplemented with a total of 1500 $\mathrm{mg} / \mathrm{d} \mathrm{Ca}$

Both groups encouraged to feed human milk for $\geq 4-6$ mo

\section{Outcome:}

Food allergy at $4 \mathrm{y}$

Period prevalence: Defined as the proportion of participants currently evidencing a measured parameter

\section{Cumulative prevalence:}

Prophylaxis vs Control: NS

\section{Key confounders accounted for:}

Age, Race/Ethnicity, SES, Smoking, Family history, HMF, CFB, Pets

(Based on Zeiger, $1989^{35}$ paper)

\section{OFCs accounted for:}

Sex

Limitations (Note: most of these limitations were identified based on Zeiger $1989^{35}$ paper)

- More women in the prophylactic-treated group withdrew before delivery because of the protocol's dietary restrictions. As a result, halfway through recruitment, randomization was changed from 40:60 (treatment: control) to $50: 50$ with a new computerized list of random numbers

- Rate of drop-out at baseline was significantly different in the prophylaxis vs. control groups $(p<0.0001)$ 
Study and Participant
Characteristics

\section{Intervention/Exposure and Outcomes Results}

Cumulative prevalence: Defined as the

proportion of subject evidencing the measured parameter at any past or current time

Prophylactic infants used casein hydrolysate (Nutramigen) for supplementation or weaning, and avoided solid foods for 6 mo; cow milk, corn, soy, citrus, and wheat, for $12 \mathrm{mo}$; and egg, peanut, and fish, for $24 \mathrm{mo}$. Infants in the control groups Enfamil, a Solid foods were introduced to controlgroup infants, based on AAP cow milk-based whey infant formula. recommendations.

Confounding, Study Limitations, and Summary of Findings

- Significantly more non-White fathers in prophylaxis group, and trend to more mothers with low education in control group

- 6 pairs of twins omitted from control group after randomization

- BW $2.9 \%$ lower in term, singletons in prophylactic group ( $\mathrm{P}=0.044)$; Overall, BW similar between groups

- Smoking during postpartum was significantly different between the prophylactic and control groups

\section{Summary:}

There was no relationship between maternal avoidance of dairy, eggs, peanut, wheat and soy during pregnancy and risk of food allergy at 3 and $4 \mathrm{y}$.

\section{Zeiger, 19954; U.S. \\ RCT \\ Baseline $\mathrm{N}=379$ Analytic $\mathrm{N}=165$}

See Zeiger, $1989^{35}$

\section{Food(s) or Food Group(s):}

- Control ( $n=106)$ : Mothers encouraged to follow standard diets during 3rd trimester of pregnancy and lactation - Prophylaxis $(n=59)$ : Avoided all milk (dairy), egg, and peanut products, concentrated soy foods (i.e., tofu), $\leq 2$ servings/d wheat during 3rd trimester of pregnancy and lactation

- Prenatal vitamins plus supplemented with a total of 1500 $\mathrm{mg} / \mathrm{d} \mathrm{Ca}$

Both groups encouraged to feed human milk for $\geq 4-6$ mo

\section{Outcome:}

Food allergy defined as atopic dermatitis, urticaria/angioedema, or

\section{Significant:}

\section{Non-significant:}

Any food-specific allergy

Period prevalence, $P=N S$

Cumulative prevalence, $\mathrm{P}=0.06$ (explained by differences $<2 y$ )

\section{Key confounders accounted for:}

Age, Race/Ethnicity, SES, Smoking, Family history, HMF, CFB, Pets

\section{OFCs accounted for:}

\section{Sex}

\section{Limitations:}

- Unclear if the participants that were lost to follow-up (since the baseline study) were any different than participants that completed the study. More participants were lost to follow up in the prophylaxis group than the control group.

- More women in the prophylactic-treated group withdrew before delivery because of the protocol's dietary restrictions. As a result, 


\section{Study and Participant}

Characteristics

\section{Intervention/Exposure and Outcomes}

Results

diarrhea/vomiting occurring on at least

two occasions or anaphylaxis induced

by a specific food with concurrent foodspecific $\lg \mathrm{E}$

at $7 \mathrm{y}$ of age

Prophylactic infants used casein hydrolysate (Nutramigen) for supplementation or weaning, and avoided solid foods for 6 mo; cow milk, corn, soy, citrus, and wheat, for $12 \mathrm{mo}$; and egg, peanut, and fish, for 24 mo. Infants in the control groups Enfamil, a cow milk-based whey infant formula.

Solid foods were introduced to controlgroup infants, based on AAP recommendations.

\section{Confounding, Study Limitations, and} Summary of Findings

halfway through recruitment, randomization was changed from 40:60 (treatment: control) to 50:50 with a new computerized list of

random numbers

- Rate of drop-out was significantly different in the prophylaxis vs. control groups $(p<0.0001)$.

- Significantly more non-White fathers in prophylaxis group, and trend to more mothers with low education in control group

- 6 pairs of twins omitted from control group after randomization

- BW 2.9\% lower in term, singletons in prophylactic group ( $\mathrm{P}=0.044)$; Overall, BW similar between groups

- Smoking during postpartum was significantly different between the prophylactic and contro groups

\section{Summary:}

Maternal avoidance of milk (dairy), egg, and peanut products, avoidance of concentrated soy foods, and limited intake of wheat during the 3rd trimester of pregnancy through lactation, and reduced infant food allergen exposure during CFB did not affect the prevalence of food allergy in the child at $7 \mathrm{y}$, replicating previously reported findings at $3 y$ and $4 \mathrm{y}$. 
Table 3. Description of evidence on the relationship between maternal diet during pregnancy and lactation and risk of child atopic dermatitis/eczema ${ }^{\text {xii, } x \text { iii }}$

\begin{tabular}{llll}
\hline $\begin{array}{l}\text { Study and Participant } \\
\text { Characteristics }\end{array}$ & $\begin{array}{l}\text { Intervention/Exposure and } \\
\text { Outcomes }\end{array}$ & Results & $\begin{array}{l}\text { Confounding, Study Limitations, and Summary } \\
\text { of Findings }\end{array}$ \\
\hline
\end{tabular}

\begin{tabular}{l}
\hline Pregnancy \\
Randomized Controlled Trials
\end{tabular}

Falth-Magnusson, $1987^{7}$ Sweden, RCT

Baseline $\mathrm{N}=212$ Analytic $\mathrm{N}=181$

Power calculation: Yes

- Age: NR

- Race/Ethnicity: NR

- SES: NR

- Significant differences by exposure: HMF, CFB

- No differences by exposure: Smoking (infant exposure), Family history, BW, Animal/Pets/Farming exposure

\section{Food(s) or Food Groups(s):}

- Non-Diet (ND): Mothers encouraged to follow standard diets, $\mathrm{n}=108$ randomized, 102 adhered and completed follow-up

- Diet (D): Strictly milk- and egg-free diet, $n=104,79$ adhered and completed follow-up

Significant:

\section{Non-Significant:}

Probable atopic dermatitis, $\mathrm{P}=\mathrm{NS}$

Definite atopic dermatitis, $\mathrm{P}=\mathrm{NS}$

- Supplemented with casein

hydrolysate formula and $\mathrm{Ca}$ supplement for a total of $1200 \mathrm{mg} / \mathrm{d}$ $\mathrm{Ca}$

from 28 wk through delivery

Both groups encouraged to feed human milk

By their own choice, many mothers limited milk and egg intake during lactation: at both 6 wk and $3 \mathrm{mo}$, D-true

\section{Key confounders accounted for:}

Family history, Animal/Pets/Farming exposure

\section{OFCs accounted for:}

None

\section{Limitations:}

- Age at weaning: D-true (7.06 mo), ND-true (5.98 $\mathrm{mo}), \mathrm{P}<0.005$; Full or partial HMF at 6 wk and 6 $\mathrm{mo}, \mathrm{P}=\mathrm{NS}$

- Infant CM introduction by 6 mo: D-true (55.1\%), ND-true (81.2\%), $\mathrm{P}<0.0005$

- Unclear whether sex was balanced: D-true (32F, 47M), ND-true (40F, 62M)

- One pair of twins included, not accounted for in analyses (group NR)

- 3 infants born preterm, all in $D$ group

- $~ 50 \%$ of women adhered to casein hydrolysate

xii \pm indicates values of Mean \pm SD unless otherwise noted

xiii AAP: American Academy of Pediatrics, ALSPAC: Avon Longitudinal Study of Parents and Children, aOR: adjusted odds ratio, aRR: adjusted risk ratio, BW: birth weight, Ca: Calcium, CFB: complementary food and beverage, Cl: confidence interval, d: day, DHQ: diet history questionnaire, DP: dietary pattern, FFQ: food frequency questionnaire, GA: gestational age, GUSTO: Growing Up in Singapore Towards Healthy Outcomes, HMF: human milk feeding, INMA: INfancia y Medio Ambiente, IQR: interquartile range, IRR: incidence rate ratio, ISAAC: International Study of Asthma and Allergies in Childhood, KOMCHS: Kyushu Okinawa Maternal and Child Health Study, MD: Mediterranean diet, mo: month(s), MoBa: Mothers and Babies cohort, MUFA: monounsaturated fatty acid, NR: not reported, NS: non-significant, OFCs: other factors considered, OMCHS: Osaka Maternal and Child Health Study, PCA: principal component analysis, PCS: prospective cohort study, Q\#: quartile, RCT: randomized control trial, Ref: Reference, SES: socioeconomic status, SFA: saturated fatty acid, SPT: skin prick test, T\#: tertile, wk: week(s), y: year(s) 


\begin{tabular}{|c|c|c|}
\hline $\begin{array}{l}\text { Study and Participant } \\
\text { Characteristics }\end{array}$ & $\begin{array}{l}\text { Intervention/Exposure and } \\
\text { Outcomes }\end{array}$ & $\begin{array}{l}\text { Confounding, Study Limitations, and Summary } \\
\text { of Findings }\end{array}$ \\
\hline $\begin{array}{l}\text { All women had a personal } \\
\text { history of allergy (asthma, } \\
\text { rhinoconjunctivitis, and atopic } \\
\text { eczema) or this was present } \\
\text { in at least one of the family } \\
\text { member (husband or child) }\end{array}$ & $\begin{array}{l}\text { group had greater proportion of } \\
\text { mothers with complete avoidance } \\
\text { (P<0.05) or low intake of } \leq 2 \mathrm{dL} / \text { wk milk } \\
\text { and } \leq 2 \text { eggs/wk ( } \mathrm{P}<0.0001) \text {. } \\
\text { Outcome: } \\
\text { Atopic dermatitis at } 18 \text { mo } \\
\text { Probable, definite } \\
\text { Outcome assessment methods: All } \\
\text { babies who demonstrated any signs } \\
\text { and symptoms suggesting atopic } \\
\text { disease or allergy before the age of } 18 \\
\text { mo were examined by a senior } \\
\text { pediatric allergist. The selection of } \\
\text { examination was based on the } \\
\text { information from the questionnaires, } \\
\text { available case records, results of two } \\
\text { skin prick tests and the result of a } \\
\text { nurse visit at } 18 \text { mo. All babies } \\
\text { demonstrating positive SPTs on any } \\
\text { occasion were examined, as well as } \\
\text { babies with a history of either otitis } \\
\text { media, bronchitis, or pseudocroup on } \\
\text { two or more occasions, and babies } \\
\text { whose parents or the nurse had } \\
\text { suspected eczema at any time. The } \\
\text { aim of the physical examination at } 18 \\
\text { mo was to score whether the child up } \\
\text { to the age of that age had } \\
\text { demonstrated definite, probable, } \\
\text { possible, or no signs of atopic } \\
\text { diseases. A question mark after atopic } \\
\text { dermatitis points out that some but not } \\
\text { all of the diagnostic features were } \\
\text { present. }\end{array}$ & $\begin{array}{l}\text { - Differences in HMF duration and CFB could have } \\
\text { influenced the outcome } \\
\text { - Attrition of } 15 \% \text { still left >180 participants required } \\
\text { by estimate from power calculation } \\
\text { - Lost to follow-up was higher in the D group than } \\
\text { the ND group } \\
\text { - High risk participants (i.e., either the mother, father } \\
\text { or another child had history of allergy). Thus, the } \\
\text { findings may not be entirely generalizable } \\
\text { - No pre-registered data analysis plan } \\
\text { Summary: } \\
\text { Maternal avoidance of milk and eggs during } 28 \text { wk } \\
\text { gestation through delivery was not associated with } \\
\text { atopic dermatitis in the child at } 18 \text { mo. }\end{array}$ \\
\hline
\end{tabular}




\begin{tabular}{|c|c|c|c|}
\hline $\begin{array}{l}\text { Study and Participant } \\
\text { Characteristics }\end{array}$ & $\begin{array}{l}\text { Intervention/Exposure and } \\
\text { Outcomes }\end{array}$ & Results & $\begin{array}{l}\text { Confounding, Study Limitations, and Summary } \\
\text { of Findings }\end{array}$ \\
\hline 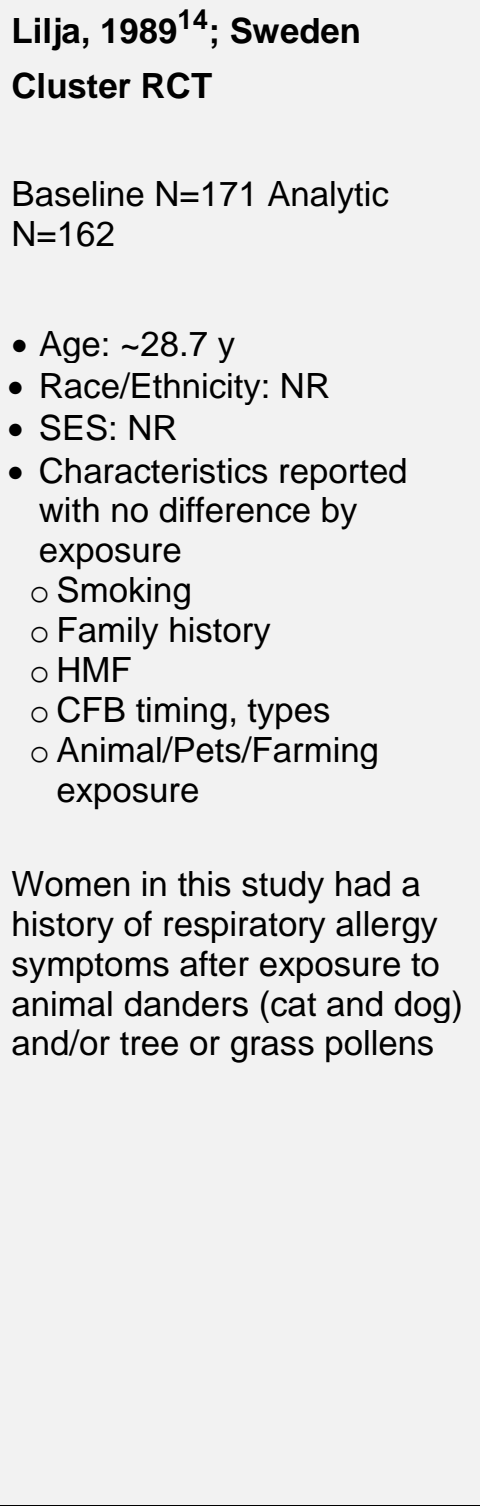 & $\begin{array}{l}\text { Food(s) or Food Group(s): } \\
\text { - High: Consumed "normal" amounts of } \\
\text { hens' egg and cows' milk, n=83 } \\
\text { - Reduced: Strictly reduced ingestion } \\
\text { of egg and dairy products (milk, } \\
\text { yogurt, butter, cheese, etc.), n=79 } \\
\text { o Reduced A-Group: Women chose to } \\
\text { continue reduced diet through } 2 \text { mo } \\
\text { postpartum ( } n=25) \\
\text { o Reduced B-Group: Women adhered } \\
\text { to study design and stopped reduced } \\
\text { diet after delivery ( } n=54 \text { ) } \\
\text { from } 28 \text { wk gestation until delivery (until } \\
2 \text { mo postpartum for A-Group) } \\
\text { Dietary assessment methods: } \\
\text { Women were randomly assigned to } \\
\text { groups. In reduced group, no foods } \\
\text { obviously containing egg or cows' milk } \\
\text { were allowed, but small amounts of } \\
\text { these foods, such as bread brushed } \\
\text { with egg or ordinary margarines, were } \\
\text { allowed. } \\
\text { Outcome: Atopic disease: Atopic } \\
\text { eczema } \\
\text { Rhinoconjunctivitis } \\
\text { at } 2,6,12 \text { and } 18 \text { mo } \\
\text { Outcome assessment methods: } \\
\text { All children were examined at } 2,6,12, \\
\text { and } 18 \text { mo. The physicians who } \\
\text { performed the physical examination at } \\
18 \text { mo were unaware of the mothers' } \\
\text { diet during the trial and of previously }\end{array}$ & $\begin{array}{l}\text { Non-significant: } \\
\text { Atopic disease by } 18 \text { mo } \\
\text { - There were no significant } \\
\text { differences between any groups } \\
\text { (High, Reduced, A-Group, B- } \\
\text { Group) in cumulative prevalence of } \\
\text { obvious, probable, or possible } \\
\text { atopic disease. } \\
\text { - Similar lack of association between } \\
\text { maternal diet and obvious, } \\
\text { probable, or possible atopic } \\
\text { eczema, rhinoconjunctivitis } \\
\text { - Similar lack of association between } \\
\text { maternal diet and obvious, } \\
\text { probable, or possible atopic } \\
\text { disease in sensitivity analyses of } \\
\text { different feeding methods during } \\
\text { the first } 6 \text { mo postpartum: } \\
\text { o HMF }>6 \text { mo } \\
\text { oHMF }>2 \text { mo/<6 mo + soy formula } \\
\text { oHMF }>2 \text { mo/<6mo + cows' } \\
\text { milk/soy formula } \\
\text { oHMF <2 mo + soy formula }\end{array}$ & $\begin{array}{l}\text { Key confounders accounted for: } \\
\text { Age, Race/Ethnicity, SES, Smoking, Family history, } \\
\text { HMF, CFB, Pets } \\
\text { OFCs accounted for: } \\
\text { Child sex } \\
\text { Limitations: } \\
\text { - There was a significantly higher incidence of } \\
\text { atopic eczema and higher IgE levels before week } \\
28 \text { in the 'reduced' group. } \\
\text { - Data < } 18 \text { mo measured but NR. } \\
\text { - } 4 \text { pairs of twins included, but family relation is not } \\
\text { accounted for in analyses. } \\
\text { - Although a cluster RCT, it is probable that the data } \\
\text { analysis was conducted at the individual level. } \\
\text { Summary: } \\
\text { Higher (vs reduced) intake of egg and dairy } \\
\text { products during pregnancy did not impact child's } \\
\text { eczema risk at } 18 \text { mo. }\end{array}$ \\
\hline
\end{tabular}


Study and Participant

Characteristics
Intervention/Exposure and

Outcomes

performed SPT and in-vitro analyses in

the mother and child. The cumulative

incidence of signs of atopic diseases

$\geq 18$ mo was evaluated by a

questionnaire and the physical

examination into the following groups:

obvious atopic, probable atopic,

possible atopic, and the type of atopic

symptom was noted.

The infants were followed with SPTs

and with serum samples for analyses of

the levels of antibodies to selected food

allergens.

\section{Prospective Cohort Studies}

Miyake, 200924; Japan PCS, OMCHS

Baseline $\mathrm{N}=1,002$ Analytic $\mathrm{N}=763$

Of the 1002 participants, 867 mother-child pairs

participated in the 2nd survey (2-9mo postpartum), 763 participated in the 3rd survey (16-24mo postpartum).

- Age: $30.0 \pm 4.0 y$

- Race/Ethnicity: NR (likely 100\% Japanese)

- SES:

- Maternal educational (y)

\section{Food(s) or Food Group(s):}

Fish consumption by quartile, $n=763$ assessed once during pregnancy (17.7 \pm 6.7 wk gestation)

Meat consumption by quartile, $n=763$ assessed once during pregnancy (17.7 \pm 6.7 wk gestation)

\section{Dietary assessment methods:}

Participants completed a selfadministered, semiquantitative, 150item comprehensive DHQ that assessed dietary habits during the preceding month. $\mathrm{DHQ}$ validated in women, and estimates of daily intake for foods were based on the Standard

\section{Significant:}

\section{Non-significant:}

\section{Eczema}

- Fish, $\mathrm{P}$ for trend=NS

- Meat, $\mathrm{P}$ for trend=NS

\section{Key confounders accounted for:}

Age, Race/Ethnicity, SES, Smoking, Family history, BW, HMF, Urban/Rural environment (residential municipality)

OFCs accounted for:

Sex

\section{Limitations:}

- Although the diet data was collected during pregnancy, there was a wide variability as to when the data was collected (range: 5-39 wk).

- Outcome measurement was subjective and was reported by the participants, who were aware of the exposure.

- Study was conducted in Japan, so the findings may not be entirely generalizable. 


\begin{tabular}{|c|c|c|c|}
\hline $\begin{array}{l}\text { Study and Participant } \\
\text { Characteristics }\end{array}$ & $\begin{array}{l}\text { Intervention/Exposure and } \\
\text { Outcomes }\end{array}$ & Results & $\begin{array}{l}\text { Confounding, Study Limitations, and Summary } \\
\text { of Findings }\end{array}$ \\
\hline $\begin{array}{l}\text { - }<13: 27.8 \% \\
\text { - 13-14: } 43.1 \% \\
\text { - } \geq 15: 29.1 \% \\
\circ \text { Annual household income } \\
\text { (yen) } \\
\text { - }<4,000,000: 27.7 \% \\
\text { - 4,000,000-5,999,999: } \\
41.0 \% \\
\text { - } \geq 6,000,000: 31.3 \% \\
\text { - Other characteristics } \\
\text { reported but no test for } \\
\text { differences by exposure: } \\
\text { o Smoking } \\
\text { o Family history of atopic } \\
\text { disease } \\
\text { o BW } \\
\text { ○ HMF }\end{array}$ & $\begin{array}{l}\text { Tables of Food Composition in Japan. } \\
\text { Surveys were completed at enrollment, } \\
\text { 17.7 wk gestation. } \\
\text { Outcome: Atopic Dermatitis: Eczema } \\
\text { at } 16-24 \text { mo } \\
\text { Outcome assessment methods: } \\
\text { Symptoms of eczema were defined as } \\
\text { in the ISAAC in a self-administered } \\
\text { questionnaire at } 16-24 \text { mo postpartum. } \\
\text { For } \\
\text { eczema, affirmative answers to the } \\
\text { following } 3 \text { questions were required: } \\
\text { "Has your child ever had an itchy rash } \\
\text { which was coming and going for at } \\
\text { least } 6 \text { months?" "Has your child had } \\
\text { this itchy rash at any time in the last } 12 \\
\text { months?" and "Has this itchy rash at } \\
\text { any time affected any of the following } \\
\text { places: the folds of the elbows, behind } \\
\text { the knees, in front of the ankles, under } \\
\text { the buttocks, or around the neck, ears, } \\
\text { or eyes?" }\end{array}$ & & $\begin{array}{l}\text { Summary: } \\
\text { Higher maternal fish or meat intake during } \\
\text { pregnancy was not associated with odds of eczema } \\
\text { in children at } 16-24 \text { mo. }\end{array}$ \\
\hline Miyake, $2010^{23}$; Japan & Food(s) or Food Group(s): & Significant: & Key confounders accounted for: \\
\hline $\begin{array}{l}\text { PCS, OMCHS } \\
\text { Baseline } N=1,002 \text { Analytic } \\
N=763\end{array}$ & $\begin{array}{l}\text { Total vegetable consumption by } \\
\text { quartile, } \mathrm{n}=763 \\
\text { Median energy-adjusted intake }(\mathrm{g} / \mathrm{d}) \text { : } \\
\text { Q1: } 90.9\end{array}$ & $\begin{array}{l}\text { Eczema } \\
\text { - Green and yellow vegetables, } P \text { for } \\
\text { trend=0.01 } \\
\circ Q 1: \text { Reference, Cases } n=54\end{array}$ & $\begin{array}{l}\text { Age, Race/Ethnicity, SES, Smoking (household), } \\
\text { Family history, BW, HMF, Urban/Rural environment } \\
\text { (residential municipality) }\end{array}$ \\
\hline See Miyake, $2009^{24}$ & $\begin{array}{l}\text { Q2: } 144.4 \\
\text { Q3: } 189.8 \\
\text { Q4: } 288.4 \\
\text { assessed once during pregnancy } \\
(\sim 17.7 \pm 6.7 \text { wk gestation) }\end{array}$ & $\begin{array}{l}\text { Q2: Cases } n=22, \text { aOR=0.30, } \\
\text { 95\% Cl: }(0.16-0.52) \\
\text { Q3: Cases } n=34, \text { aOR=0.53, } \\
\text { 95\% Cl: }(0.31-0.89) \\
\text { Q4: Cases } n=32, \text { aOR }=0.41 \\
\text { 95\% Cl: }(0.24-0.71)\end{array}$ & $\begin{array}{l}\text { OFCs accounted for: } \\
\text { Sex } \\
\text { Limitations: }\end{array}$ \\
\hline
\end{tabular}


Study and Participant

\section{Intervention/Exposure and} Outcomes

Total fruit consumption by quartile, $\mathrm{n}=763$

Median energy-adjusted intake ( $\mathrm{g} / \mathrm{d})$ :

Q1: 49.6

Q2: 114.3

Q3: 176.4

Q4: 290.8

assessed once during pregnancy ( 17.7 6.7 wk gestation)

Also examined outcomes by intake (by quartiles) of green and yellow vegetables; other vegetables; apples; and citrus fruit

\section{Dietary assessment methods:}

Participants completed a selfadministered, semiquantitative, 150item comprehensive DHQ that assessed dietary habits during the preceding month. DHQ validated in women 31-69y, and estimates of daily intake for foods were based on the Standard Tables of Food Composition in Japan. Surveys were completed at enrollment, 17.7 wk gestation.

Green and yellow vegetable

consumption included intake of carrots, pumpkin, tomatoes, tomato juice, sweet peppers, broccoli, and dark-green leafy vegetables such as spinach and komatsuna. Intake of other vegetables

\section{Results}

- Citrus fruit, $P$ for trend=0.03

- Q1: Reference, Cases $n=49$

- Q2: Cases $\mathrm{n}=34, \mathrm{aOR}=0.61$, 95\% Cl: (0.36-1.02)

- Q3: Cases $\mathrm{n}=31, \mathrm{aOR}=0.57$, 95\% Cl: (0.33-0.98)

- Q4: Cases $n=28, a O R=0.53$, 95\% Cl: (0.30-0.93)

\section{Non-significant:}

Eczema

- Total vegetables, $P$ for trend=NS

- Other vegetables, $P$ for trend=NS

- Total fruit, $P$ for trend=NS

- Apples, P for trend=NS
Confounding, Study Limitations, and Summary of Findings

- Although the diet data was collected during pregnancy, there was a wide variability as to when the data was collected (range: 5-39 wk).

- Outcome measurement was subjective and was reported by the participants, who were aware of the exposure.

- Study was conducted in Japan, so the findings may not be entirely generalizable.

\section{Summary:}

Higher maternal intake of green and yellow vegetables and citrus fruit during pregnancy was associated with lower odds of eczema in children at 16-24 mo.

Higher maternal intake of total vegetables, other vegetables, total fruit, and apples during pregnancy was not associated with odds of eczema in children at $16-24 \mathrm{mo}$ 
Study and Participant

Characteristics
Intervention/Exposure and

Outcomes

included that of cabbage, cucumber,

lettuce, Chinese cabbage, bean

sprouts, Japanese radish, onion,

cauliflower, eggplant, burdock, lotus

root, vegetable juice, umeboshi, and

salt pickles. Fruit consumption was

defined as intake of raisins, canned

fruits, fruit juice, citrus fruit, bananas,

apples, strawberries, grapes, peaches,

Japanese pears, Japanese

persimmons, kiwifruit, melon, and

watermelon. Because only a small

number of participants used vitamin C

$(5.6 \%)$ and multivitamin $(4.2 \%)$

supplements $\geq 1 / \mathrm{wk}$, information on

these dietary supplements was not

considered in the present analysis.

Outcome: Atopic Dermatitis: Eczema

\section{at $16-24 \mathrm{mo}$}

Outcome assessment methods:

See Miyake, $2009^{24}$
Confounding, Study Limitations, and Summary of Findings

\section{Miyake, 201022; Japan} PCS, OMCHS

See Miyake, $2009^{24}$

\section{Food(s) or Food Group(s):}

Total dairy products consumption by quartile, $n=763$

Median energy-adjusted intake (g/d):
Q1: 43.6
Q2: 120.8
Q3: 184.5
Q4: 280.7

Results

\section{Significant:}

\section{Non-significant:}

Eczema

- Total dairy products, $\mathrm{P}$ for trend=NS

- Milk, P for trend=NS

- Yogurt, $\mathrm{P}$ for trend=NS

- Cheese, $\mathrm{P}$ for trend=NS

\section{Key confounders accounted for:}

Age, Race/Ethnicity, SES, Smoking, Family history, BW, HMF, Urban/Rural environment (residential municipality)

\section{OFCs accounted for:}

Sex

Limitations: 
Study and Participant

Characteristics
Intervention/Exposure and

Outcomes

Results

assessed once during pregnancy

( 17.7 \pm 6.7 wk gestation)

Also examined outcomes by intake (by quartiles) of milk; yogurt; and cheese

\section{Dietary assessment methods:}

Participants completed a selfadministered, semiquantitative, 150-

item comprehensive DHQ that

assessed dietary habits during the preceding month. $\mathrm{DHQ}$ validated in women 31-69y, and estimates of daily intake for foods were based on the Standard Tables of Food Composition in Japan. Surveys were completed at enrollment, 17.7wk gestation.

Total dairy product intake was considered as the sum of milk, yogurt and cheese. Information on dietary supplements was not used because only a small number of participants used calcium (5.5\%) and multivitamin $(4.2 \%)$ supplements $\geq 1 / \mathrm{wk}$

Outcome: Atopic Dermatitis: Eczema

at $16-24 \mathrm{mo}$

Outcome assessment methods:

See Miyake, $2009^{24}$
Confounding, Study Limitations, and Summary of Findings

- Although the diet data was collected during pregnancy, there was a wide variability as to when the data was collected (range: 5-39wk).

- Outcome measurement was subjective and was reported by the participants, who were aware of the exposure.

- Study was conducted in Japan, so the findings may not be entirely generalizable.

\section{Summary:}

Higher maternal intake of total dairy products, milk, yogurt, and cheese during pregnancy was not associated with odds of eczema in children at 16-24 mo.

Key confounders accounted for: 
Study and Participant

Characteristics

\section{PCS, OMCHS}

Baseline $\mathrm{N}=1,002$ Analytic $\mathrm{N}=763$

See Miyake, $2009^{24}$

\section{Intervention/Exposure and}

Outcomes

Dietary Pattern, by quartiles of

adherence

- Healthy: represented higher intake of green and yellow vegetables, seaweed, mushrooms, white vegetables, pulses, potatoes, fish, sea products, fruit, and shellfish and lower intake of confectioneries and soft drinks

- Western: high intake of vegetable oil, salt-containing seasonings, beef and pork, processed meat, eggs, chicken, and white vegetables and low intake of fruit, soft drinks, and confectioneries

- Japanese: high intake of rice, miso soup, sea products, and fish and low intake of bread, confectioneries, and dairy products

assessed once during pregnancy

( 17.7 \pm 6.7 wk gestation)

\section{Dietary assessment methods:}

Participants completed a selfadministered, semiquantitative, 150item comprehensive DHQ that assessed dietary habits during the preceding month. $\mathrm{DHQ}$ validated in women 31-69 y, and estimates of daily intake for foods were based on the Standard Tables of Food Composition in Japan. Surveys were completed at enrollment, 17.7 wk gestation.

\section{Results}

\section{Non-significant:}

\section{Eczema}

- Healthy pattern, $P$ for trend=NS

- Western pattern, $P$ for trend=NS

- Japanese pattern, $P$ for trend=NS

Results of the sensitivity analysis were consistent with the main findings.
Confounding, Study Limitations, and Summary of Findings

Age, Race/Ethnicity, SES, Smoking, Family history, BW, HMF, Urban/Rural environment (residential municipality)

\section{OFCs accounted for:}

Sex

\section{Limitations:}

- Although the diet data was collected during pregnancy, there was a wide variability as to when the data was collected (range: 5-39wk).

- Outcome measurement was subjective and was reported by the participants, who were aware of the exposure.

- Study was conducted in Japan, so the findings may not be entirely generalizable.

\section{Summary:}

Adherence to Healthy, Western, or Japanese dietary patterns during pregnancy was not associated with odds of eczema in children at 16-24 mo. 
Study and Participant

Characteristics
Intervention/Exposure and

Outcomes

to extract dietary patterns. Five items were excluded (cornflakes, nutritional supplement bars, Japanese-style pancakes, noodle soup, and drinking water). Information on dietary supplements was not used in the calculation of dietary intake.

Dietary patterns obtained via factor analysis. Scores for each pattern and for each individual were determined by summing the intake of each food group weighted by the factor loading. All data presented here are from the Varimax rotation. The validity of dietary patterns derived from the DHQ has been previously reported.

Outcome: Atopic Dermatitis: Eczema

at $16-24$ mo

Outcome assessment methods:

See Miyake, $2009^{24}$

\section{Results}

Confounding, Study Limitations, and Summary of Findings

\section{Saito, $2010^{28}$; Japan} PCS, OMCHS

Baseline $\mathrm{N}=1002$ Analytic $\mathrm{N}=771$

Of the 1002 participants, 867 mother-child pairs

participated in the $2^{\text {nd }}$ survey

(2-9 mo postpartum). After

further excluding 96

\section{Food(s) or Food Group(s): \\ Significant:}

Dairy product consumption by quartile, $\mathrm{n}=771$

Median energy-adjusted intake ( $\mathrm{g} / \mathrm{d})$ :

Q1: 52.7

Q2: 126.0

Q3: 191.0

Q4: 288.3
Eczema

- Meat, $P$ for trend=0.01

- Q1: Reference, Cases $n=10$

○ Q2: Cases $n=14, a O R=1.46$, 95\% Cl: (0.61-3.62)

- Q3: Cases $n=19, a O R=2.41$, 95\% Cl: (1.06-5.75)

○ Q4: Cases $n=22$, aOR=2.59, $95 \% \mathrm{Cl}:(1.15-6.17)$

\section{Key confounders accounted for:}

Age, Race/Ethnicity, SES, Family history, BW, HMF, Urban/Rural environment (residential municipality)

\section{OFCs accounted for:}

Sex, Indoor/outdoor environment

\section{Limitations:}




\begin{tabular}{|c|c|c|c|}
\hline $\begin{array}{l}\text { Study and Participant } \\
\text { Characteristics }\end{array}$ & $\begin{array}{l}\text { Intervention/Exposure and } \\
\text { Outcomes }\end{array}$ & Results & $\begin{array}{l}\text { Confounding, Study Limitations, and Summary } \\
\text { of Findings }\end{array}$ \\
\hline $\begin{array}{l}\text { participants, the final sample } \\
\text { size was } 771 \text {. } \\
\text { - Age: } 29.9 \pm 4.0 y \\
\text { - Race/Ethnicity: NR (likely } \\
\text { 100\% Japanese) } \\
\text { - SES: } \\
\text { o Maternal educational (y) } \\
\text { - }<13: 28.7 \% \\
\text { - 13-14: } 42.4 \% \\
\text { - } \geq 15: 28.9 \% \\
\text { onnual household income } \\
\text { (yen) } \\
\text { - <4,000,000: } 29.1 \% \\
\text { - 4,000,000 - } 5,999,999: \\
39.7 \% \\
\text { - } \geq 6,000,000: 31.3 \% \\
\text { - Other characteristics } \\
\text { reported but no test for } \\
\text { differences by exposure: } \\
\text { o Family history of atopic } \\
\text { disease } \\
\text { o BW } \\
\text { o HMF }\end{array}$ & 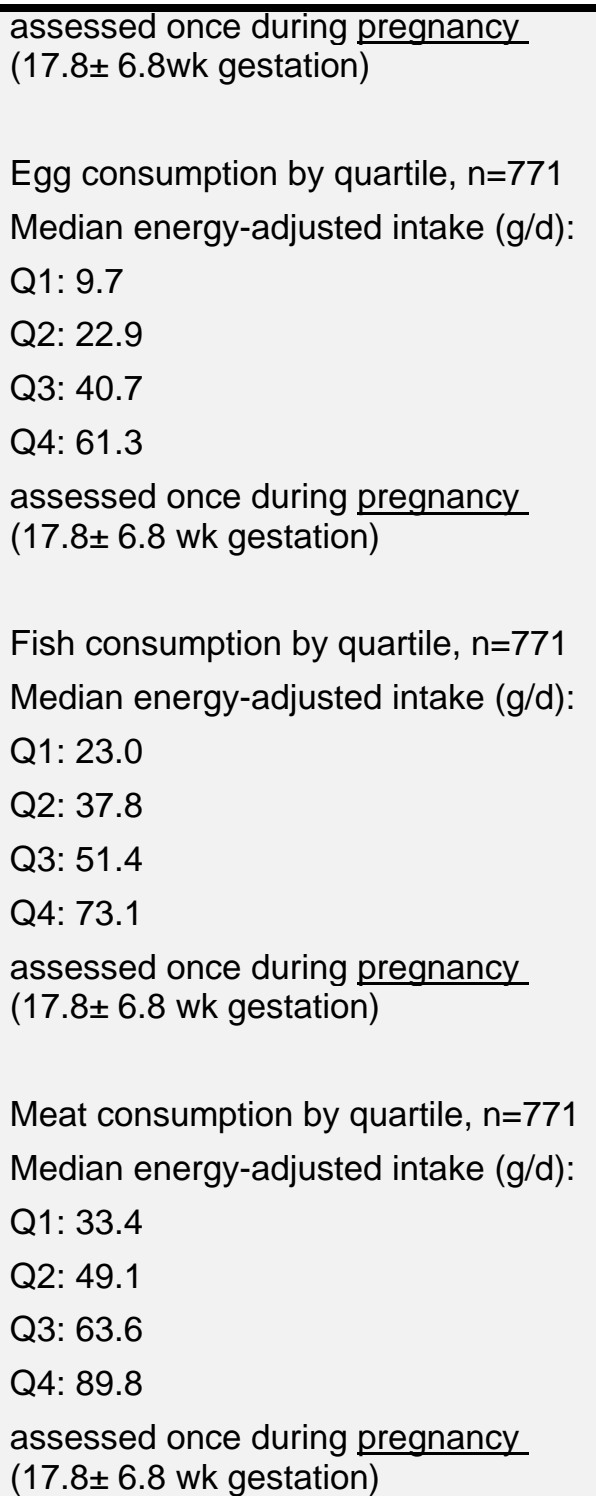 & $\begin{array}{l}\text { Non-significant: } \\
\text { Eczema } \\
\text { - Dairy products, } P \text { for trend=NS } \\
\text { - Eggs, P for trend=NS } \\
\text { - Fish, P for trend=NS }\end{array}$ & $\begin{array}{l}\text { - Although the diet data was collected during } \\
\text { pregnancy, there was a wide variability as to when } \\
\text { the data was collected (range: } 5-39 \text { wk). } \\
\text { - Outcome measurement was subjective and was } \\
\text { reported by the participants, who were aware of } \\
\text { the exposure. } \\
\text { - Study was conducted in Japan, so the findings } \\
\text { may not be entirely generalizable. } \\
\text { Summary: } \\
\text { Higher meat intake during pregnancy was } \\
\text { associated with greater odds of eczema in children } \\
\text { at } 3-4 \text { mo. There was no significant association } \\
\text { between intake of dairy products, eggs, or fish } \\
\text { during pregnancy and odds of eczema in the child. }\end{array}$ \\
\hline
\end{tabular}


Study and Participant

Characteristics
Intervention/Exposure and

Outcomes
Results

Confounding, Study Limitations, and Summary of Findings

\section{Dietary assessment methods:}

Participants completed a selfadministered, semiquantitative, 150item comprehensive DHQ that assessed dietary habits during the preceding month. $\mathrm{DHQ}$ validated in women, and estimates of daily intake for foods were based on the Standard Tables of Food Composition in Japan. Surveys were completed at enrollment, $\sim 17.8$ wk gestation.

Outcome: Atopic Dermatitis: Eczema at 3-4 mo

\section{Outcome assessment methods:}

Symptoms of eczema were assessed with a self-administered questionnaire at 3-4 mo postpartum. Suspected atopic eczema was considered to be present if the mother selected any one of the following answers to the written question: "Has your child been diagnosed by a physician as having atopic eczema and treated with topical steroids?": (i) my child has been diagnosed with atopic eczema and treated with topical steroids ( $n=23)$; (ii) my child has been diagnosed with atopic eczema but has not been treated with topical steroids $(n=11)$; (iii) my child has been diagnosed with atopic eczema and treated with a unknown ointment $(n=1)$; (iv) my child has been 


\begin{tabular}{|c|c|c|c|}
\hline $\begin{array}{l}\text { Study and Participant } \\
\text { Characteristics }\end{array}$ & $\begin{array}{l}\text { Intervention/Exposure and } \\
\text { Outcomes }\end{array}$ & Results & $\begin{array}{l}\text { Confounding, Study Limitations, and Summary } \\
\text { of Findings }\end{array}$ \\
\hline & $\begin{array}{l}\text { diagnosed as possibly having atopic } \\
\text { eczema and has been treated with } \\
\text { topical steroids }(n=29) \text { or }(v) \text { my child } \\
\text { has been diagnosed as possibly having } \\
\text { atopic eczema but has not been treated } \\
\text { with topical steroids }(n=1) \text {. }\end{array}$ & & \\
\hline Miyake, $2014^{26}$; Japan & Food(s) or Food Group(s): & Significant: & Key confounders accounted for: \\
\hline PCS, KOMCHS & $\begin{array}{l}\text { Dairy product consumption by quartile, } \\
\mathrm{n}=1,354 \\
\text { assessed once during pregnancy (5-39 }\end{array}$ & $\begin{array}{l}\text { Physician-diagnosed atopic eczema } \\
\text { Total yogurt products, } \mathrm{P} \text { for } \\
\text { trend }=0.01\end{array}$ & $\begin{array}{l}\text { Age, Race/Ethnicity, SES, Smoking, Family history, } \\
\text { BW, HMF }\end{array}$ \\
\hline $\begin{array}{l}\text { N=1,354 } \\
\text { - Age: } 31.5 \pm 4.1 y \\
\text { - Race/Ethnicity: NR (likely } \\
\text { 100\% Japanese) } \\
\text { - SES: } \\
\text { o Maternal educational (y) } \\
\text { - <13: } 21.6 \% \\
\text { - 13-14: } 33.6 \% \\
\text { - } \geq 15: 44.8 \% \\
\text { o Annual household income } \\
\text { (yen) } \\
\text { - <4,000,000: } 33.5 \% \\
\text { - } 4000000 \text {. } 999999 .\end{array}$ & $\begin{array}{l}\text { Dietary assessment methods: } \\
\text { Participants completed a self- } \\
\text { administered, semiquantitative, 150- } \\
\text { item comprehensive DHQ that } \\
\text { assessed dietary habits during the } \\
\text { preceding month. Surveys were } \\
\text { completed at enrollment, between } 5 \\
\text { and } 39 \text { wk gestation. Research } \\
\text { technicians completed missing or } \\
\text { illogical data by telephone interview. } \\
\text { Total dairy product intake was defined } \\
\text { as the sum of the calculated daily } \\
\text { intake of milk, yogurt, and cheese. }\end{array}$ & $\begin{array}{l}\text { - Q1: Reference, Cases } n=16 \\
\text { - Q2: Cases } n=27, a O R=1.52,95 \% \\
\text { Cl: }(0.78-3.04) \\
\text { Q3: Cases } n=10, a O R=0.48,95 \% \\
\text { Cl: }(0.20-1.10) \\
\text { - Q4: Cases } n=9, \text { aOR }=0.49,95 \% \\
\text { Cl: }(0.20-1.16) \\
\text { Sensitivity analysis showed } \\
\text { consistent findings for physician } \\
\text { diagnosed atopic eczema. } \\
\text { Non-significant: }\end{array}$ & $\begin{array}{l}\text { OFCs accounted for: } \\
\text { Sex } \\
\text { Limitations: } \\
\text { - Although the diet data were collected during } \\
\text { pregnancy, there was a wide variability as to when } \\
\text { the data was collected (range: } 5-39 \text { wk). } \\
\text { - Outcome measurement was subjective and was } \\
\text { reported by the participants, who were aware of } \\
\text { the exposure. } \\
\text { - Study was conducted in Japan, so the findings } \\
\text { may not be entirely generalizable. }\end{array}$ \\
\hline $\begin{array}{l}36.7 \% \\
-\geq 6,000,000: 29.8 \% \\
\text { - Other characteristics } \\
\text { reported but no test for } \\
\text { differences by exposure: } \\
\text { o Smoking } \\
\text { o Family history of atopic } \\
\text { disease } \\
\circ \text { BW } \\
\circ \mathrm{HMF}\end{array}$ & $\begin{array}{l}\text { Outcome: Atopic Dermatitis: Eczema } \\
\text { at } \sim 24 \text { mo } \\
\text { Outcome assessment methods: } \\
\text { Symptoms of eczema were defined as } \\
\text { in the ISAAC in a self-administered } \\
\text { questionnaire at } ~ 24 \text { mo postpartum. } \\
\text { For eczema, affirmative answers to the } \\
\text { following } 3 \text { questions were required: } \\
\text { "Has your child ever had an itchy rash } \\
\text { which was coming and going for at } \\
\text { least } 6 \text { months?" "Has your child had }\end{array}$ & $\begin{array}{l}\text { Eczema } \\
\text { - Total dairy products, } P \text { for } \\
\text { trend=0.054 } \\
\circ \mathrm{Q} 1: \text { Reference, Cases } \mathrm{n}=69 \\
\circ \mathrm{Q} 2: \text { Cases } \mathrm{n}=57, \text { aOR=0.80, } \\
\text { 95\% Cl: }(0.54-1.19) \\
\circ \mathrm{Q} 3: \text { Cases } \mathrm{n}=57, \mathrm{aOR}=0.82 \\
\text { 95\% Cl: }(0.55-1.22) \\
\circ \mathrm{Q} 4: \text { Cases } n=46, \text { aOR }=0.64 \\
\text { 95\% Cl: }(0.42-0.98)\end{array}$ & $\begin{array}{l}\text { Summary: } \\
\text { Higher maternal yogurt intake, but not milk, cheese, } \\
\text { or total dairy intake, during pregnancy was } \\
\text { associated with lower odds of physician-diagnosed } \\
\text { atopic eczema in children by } 24 \text { mo. } \\
\text { Higher maternal total dairy, milk, yogurt, or cheese } \\
\text { intake during pregnancy was not associated with } \\
\text { odds of eczema in children by } 24 \text { mo. }\end{array}$ \\
\hline
\end{tabular}


Study and Participant

Characteristics

\section{Intervention/Exposure and}

Outcomes

this itchy rash at any time in the last 12 months?" and "Has this itchy rash at any time affected any of the following places: the folds of the elbows, behind the knees, in front of the ankles, under the buttocks, or around the neck, ears, or eyes?" In addition, physiciandiagnosed atopic eczema was considered present if the child had been diagnosed by a physician as having atopic eczema at any time since birth.

\section{Food(s) or Food Group(s):}

Fish consumption by quartile, $n=1,354$ assessed once during pregnancy

Meat consumption by quartile, $n=1,354$ assessed once during pregnancy (5-39 wk gestation)

\section{Dietary assessment methods:}

See Miyake, $2014^{26}$

Outcome: Atopic Dermatitis: Eczema at $\sim 24 \mathrm{mo}$

Outcome assessment methods:

See Miyake, $2014^{26}$

\section{Results}

- Intake of milk, yogurt, and cheese individually by quartile showed similar patterns of results, and all trends were NS.

Physician-diagnosed atopic eczema

- Total dairy products, $\mathrm{P}$ for trend=NS

- Intake of milk, and cheese individually by quartile showed similar patterns of results, and trends were NS.

\section{Significant:}

\section{Non-significant:}

Eczema

- Meat, $\mathrm{P}$ for trend=NS

- Fish, $P$ for trend=0.06

○ Q1: Reference, Cases n=55

- Q2: Cases $n=43$, aOR $=0.72$, 95\% Cl: $(0.46-1.12)$

- Q3: Cases $n=63, \mathrm{aOR}=1.18$, $95 \% \mathrm{Cl}:(0.78-1.77)$

- Q4: Cases $n=68, a O R=1.29$, $95 \% \mathrm{Cl}:(0.86-1.93)$

\section{Key confounders accounted for:}

Age, Race/Ethnicity, SES, Smoking, Family history, BW, HMF

\section{OFCs accounted for:}

Sex

\section{Limitations:}

Although the diet data were collected during pregnancy, there was a wide variability as to when the data was collected (range: 5-39 wk).

- Outcome measurement was subjective and was reported by the participants, who were aware of the exposure.

- Study was conducted in Japan, so the findings may not be entirely generalizable to the U.S. population.

\section{Summary:}

Higher maternal fish or meat intake during pregnancy was not associated with odds of eczema in children by $24 \mathrm{mo}$. 


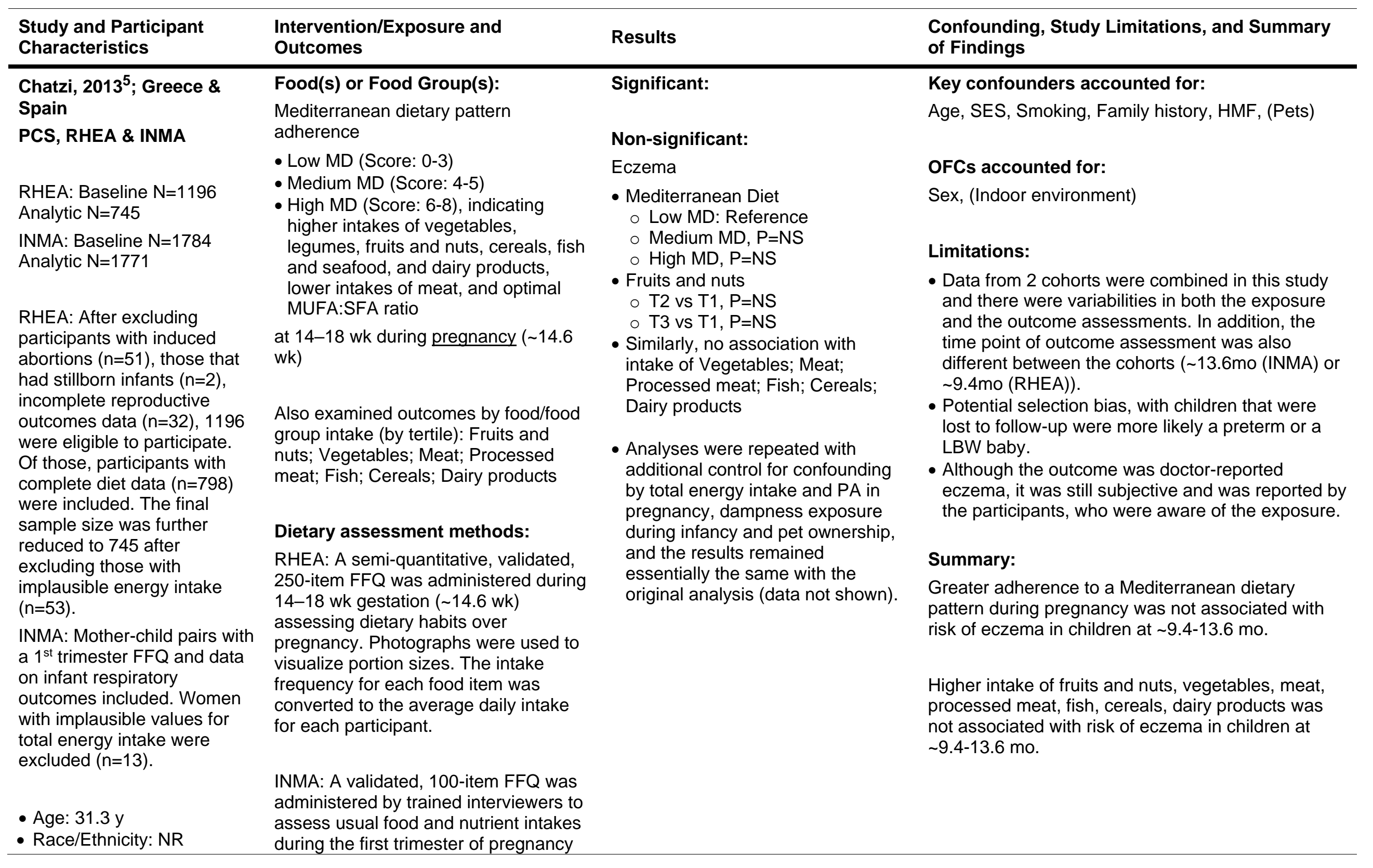




\section{Study and Participant}

Characteristics

\section{- SES:}

- Maternal education

- Low: $22.4 \%$

- Medium: $43.8 \%$

- High: 33.8\%

- Characteristics reported but no test for differences by

exposure

o Smoking

o Family history of eczema, asthma

○ GA

$\circ \mathrm{BW}$

o HMF

o Pet exposure
Intervention/Exposure and

Outcomes

to capture intake from the last

menstrual period until the time of the

interview ( 13.8 wk gestation). The

intake frequency for each food item

was converted to the average daily

intake for each participant.

MD adherence scored via EPIC

methods. 1pt awarded for intake above the median for beneficial components (vegetables, legumes, fruits and nuts, cereals, fish and seafood, and dairy products), $1 \mathrm{pt}$ for intake below the median for detrimental components (meat, including all types of meat), and $1 \mathrm{pt}$ for optimal MUFA:SFA ratio.

Otherwise, Opt awarded for each component. Alcohol not included. Total score: $0-8$.

Outcome: Atopic Dermatitis: Eczema at $\sim 13.6$ mo (INMA) or $\sim 9.4$ mo (RHEA)

\section{Outcome assessment methods:}

Based on the ISAAC phase-I questionnaire: Eczema was defined as a positive answer to the question 'Has a doctor ever told you that your child has had atopic dermatitis/ eczema?'.
Results

Confounding, Study Limitations, and Summary of Findings

\begin{tabular}{llll}
\hline Leermakers, $\mathbf{2 0 1 3}^{\mathbf{1 3}}$; The & Food(s) or Food Group(s): & Significant: & Key confounders accounted for: \\
Netherlands & Total fish intake $(\mathrm{g} / \mathrm{wk})$ & Age, Race/ethnicity, SES, Smoking, Family history, \\
PCS, Generation R & $\bullet 0: n=523($ Ref $)$ & Non-significant: \\
& $\bullet 1-69: n=728$ & GA, BW, HMF, CFB timing, Pets \\
\hline
\end{tabular}




\begin{tabular}{|c|c|c|c|}
\hline $\begin{array}{l}\text { Study and Participant } \\
\text { Characteristics }\end{array}$ & $\begin{array}{l}\text { Intervention/Exposure and } \\
\text { Outcomes }\end{array}$ & Results & $\begin{array}{l}\text { Confounding, Study Limitations, and Summary } \\
\text { of Findings }\end{array}$ \\
\hline 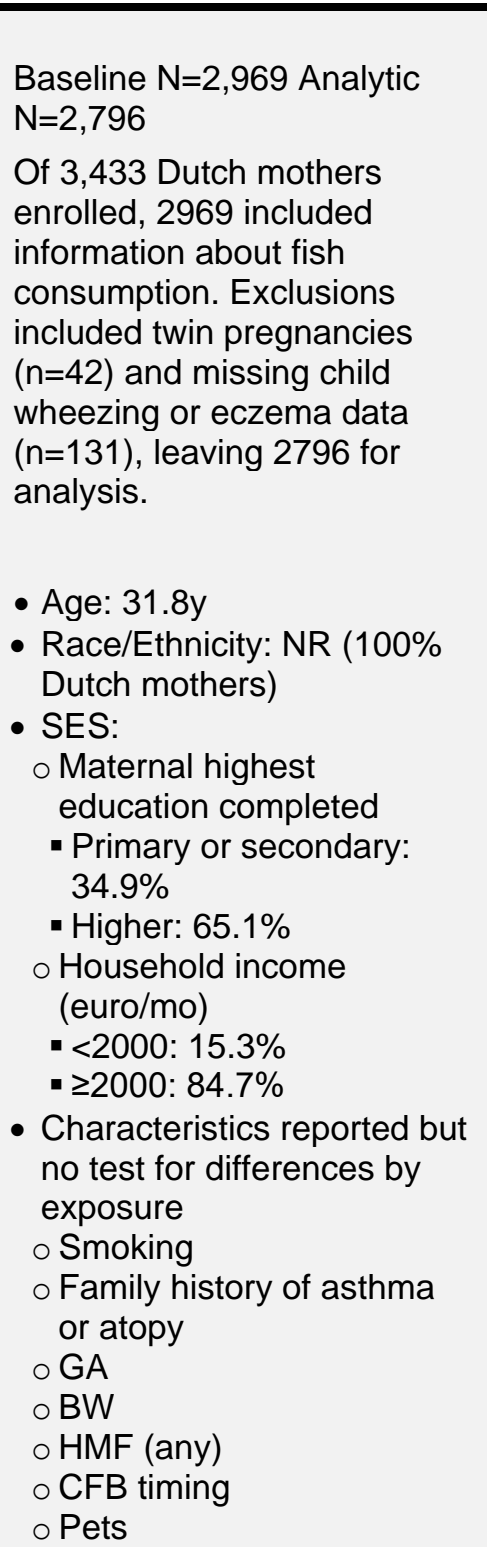 & $\begin{array}{l}\text { - 70-139: } \mathrm{n}=908 \\
\text { - 140-209: } \mathrm{n}=442 \\
\text { - }>210: \mathrm{n}=195 \\
\text { at 10.1-21.8 wk ( } 13.4 \mathrm{wk} \text { ) during } \\
\text { pregnancy } \\
\text { Also examined outcomes by intake } \\
\text { (g/wk) of: lean fish (4 levels), fatty fish } \\
\text { (4 levels), and shellfish ( } 3 \text { levels) } \\
\text { Dietary assessment methods: } \\
\text { Dietary intake assessed at } \sim 13.4 \text { wk } \\
\text { gestation using a validated, } 293 \text {-item } \\
\text { FFQ that covered dietary intake over } \\
\text { the previous } 3 \text { months. } \\
\text { Outcome: Atopic Dermatitis: Eczema } \\
\text { at } 1,2,3 \text {, and } 4 \text { y } \\
\text { Outcome assessment methods: } \\
\text { Doctor-attended eczema. Based on the } \\
\text { ISAAC questionnaire: Doctor-attended } \\
\text { eczema at age } 1 \text { refers to eczema } \\
\text { between the ages } 6 \text { to } 12 \text { months. }\end{array}$ & $\begin{array}{l}\text { Eczema } \\
\text { - Total Fish } \\
\circ \text { Age } 1 \text { y, P for trend=NS } \\
\circ \text { Similarly, no association with } \\
\text { intake at age } 2 \text { y, } 3 \text { y, } 4 \text { y or } \\
\text { overall risk of eczema } \\
\text { - Similarly, no association with } \\
\text { overall risk or risk of eczema at } \\
\text { ages } 1-4 \text { y with intake of lean fish, } \\
\text { fatty fish, or shellfish }\end{array}$ & $\begin{array}{l}\text { OFCs accounted for: } \\
\text { Sex, Maternal alcohol use } \\
\text { Limitations: } \\
\text { - Although the outcome was 'doctor-attended } \\
\text { eczema,' it was still self-reported. } \\
\text { - Generalizability may be an issue, given than the } \\
\text { study focused on } 100 \% \text { Dutch women. } \\
\text { Summary: } \\
\text { Higher intakes of total fish, lean fish, fatty fish, or } \\
\text { shellfish during pregnancy were not associated with } \\
\text { overall eczema risk and yearly risk at age } 1-4 \mathrm{y} \text {. }\end{array}$ \\
\hline
\end{tabular}




\begin{tabular}{|c|c|c|c|}
\hline $\begin{array}{l}\text { Study and Participant } \\
\text { Characteristics }\end{array}$ & $\begin{array}{l}\text { Intervention/Exposure and } \\
\text { Outcomes }\end{array}$ & Results & $\begin{array}{l}\text { Confounding, Study Limitations, and Summary } \\
\text { of Findings }\end{array}$ \\
\hline $\begin{array}{l}\text { Nguyen, } 2017^{27} \text {; The } \\
\text { Netherlands } \\
\text { PCS, Generation R } \\
\text { Baseline N=5,225 Analytic } \\
\mathrm{N}=3600 \\
\text { - Age: Median=31.7 y, } \\
\text { IQR=28.4-34.4 y } \\
\text { - Race/Ethnicity: NR for } \\
\text { mother } \\
\text { - SES: Higher educational } \\
\text { level: } 52.7 \% \text {, Household } \\
\text { income } \geq 2200 \text { Euros/mo: } \\
61.2 \% \\
\text { - Other characteristics } \\
\text { reported but no test for } \\
\text { differences by exposure } \\
\text { o Smoking } \\
\text { o Family history of eczema, } \\
\text { asthma } \\
\text { o HMF } \\
\text { o Animals/Pets/Farming } \\
\text { exposure }\end{array}$ & $\begin{array}{l}\text { Food(s) or Food Group(s): } \\
\text { Diet Quality Score } \\
\text { Higher diet quality included: } \\
\text { - High intake of vegetables, fruit, whole } \\
\text { grains, legumes, nuts, dairy, fish, and } \\
\text { tea } \\
\text { - High ratio whole grains to total grains } \\
\text { and soft fats (i.e. soft margarines) and } \\
\text { oils to total fat } \\
\text { - Low intake of red meat, sugar- } \\
\text { containing beverages, alcohol, and } \\
\text { salt } \\
\text { - Folic acid supplement use in early } \\
\text { pregnancy } \\
\text { Dietary assessment methods: } \\
\text { Validated FFQ in early pregnancy } \\
\text { (median } 13.6 \text { wks gestation } \\
\text { (IQR=12.4-16.2wks). FFQ included } \\
\text { foods frequently consumed in the } \\
\text { Dutch population and was modified for } \\
\text { use in pregnant women. Previously } \\
\text { developed predefined food-based diet } \\
\text { quality score was applied, reflecting } \\
\text { adherence to Dutch dietary guidelines. } \\
\text { Diet quality score included continuous } \\
\text { scores on } 15 \text { components: vegetables, } \\
\text { fruit, whole grains, legumes, nuts, } \\
\text { dairy, fish, tea, ratio whole grains of } \\
\text { total grains, ratio soft fats (i.e., soft } \\
\text { margarines) and oils of total fat, red } \\
\text { meat, sugar-containing beverages, } \\
\text { alcohol, salt, and folic acid supplement } \\
\text { use in early pregnancy. Maximum } \\
\text { score for each component was } 1 \text {, with } \\
\text { an overall score ranging from } 0 \text { to } 15 . \text { A }\end{array}$ & $\begin{array}{l}\text { Non-significant: } \\
\text { Risk of ever eczema at } 10 \text { y per } 1 \\
\text { point higher diet quality score }(\mathrm{n} \\
=3,600), \mathrm{P}=\mathrm{NS} \\
\text { Results of a sensitivity analysis were } \\
\text { consistent with the main findings }\end{array}$ & $\begin{array}{l}\text { Key confounders accounted for: } \\
\text { SES, Smoking, Family history, HMF, } \\
\text { Animals/pets/farming exposure, (race/ethnicity, only } \\
\text { in a sensitivity analysis) } \\
\text { OFCs accounted for: } \\
\text { Child sex, Maternal substance use } \\
\text { Limitations: } \\
\text { - At least one key confounder not controlled for } \\
\text { - Methods used to assess the exposure likely to } \\
\text { result in minimal exposure misclassification } \\
\text { - Proportions of and reasons for missing } \\
\text { participants may differ across exposure groups } \\
\text { and the analysis is unlikely to have removed the } \\
\text { risk of bias arising from the missing data } \\
\text { - Outcomes were subjective and assessed by } \\
\text { participants, who were aware of the exposure } \\
\text { received } \\
\text { - Limited generalizability } \\
\text { - No pre-registered data analysis plan } \\
\text { Summary: } \\
\text { Diet quality during pregnancy was not associated } \\
\text { with risk of eczema in childhood. }\end{array}$ \\
\hline
\end{tabular}




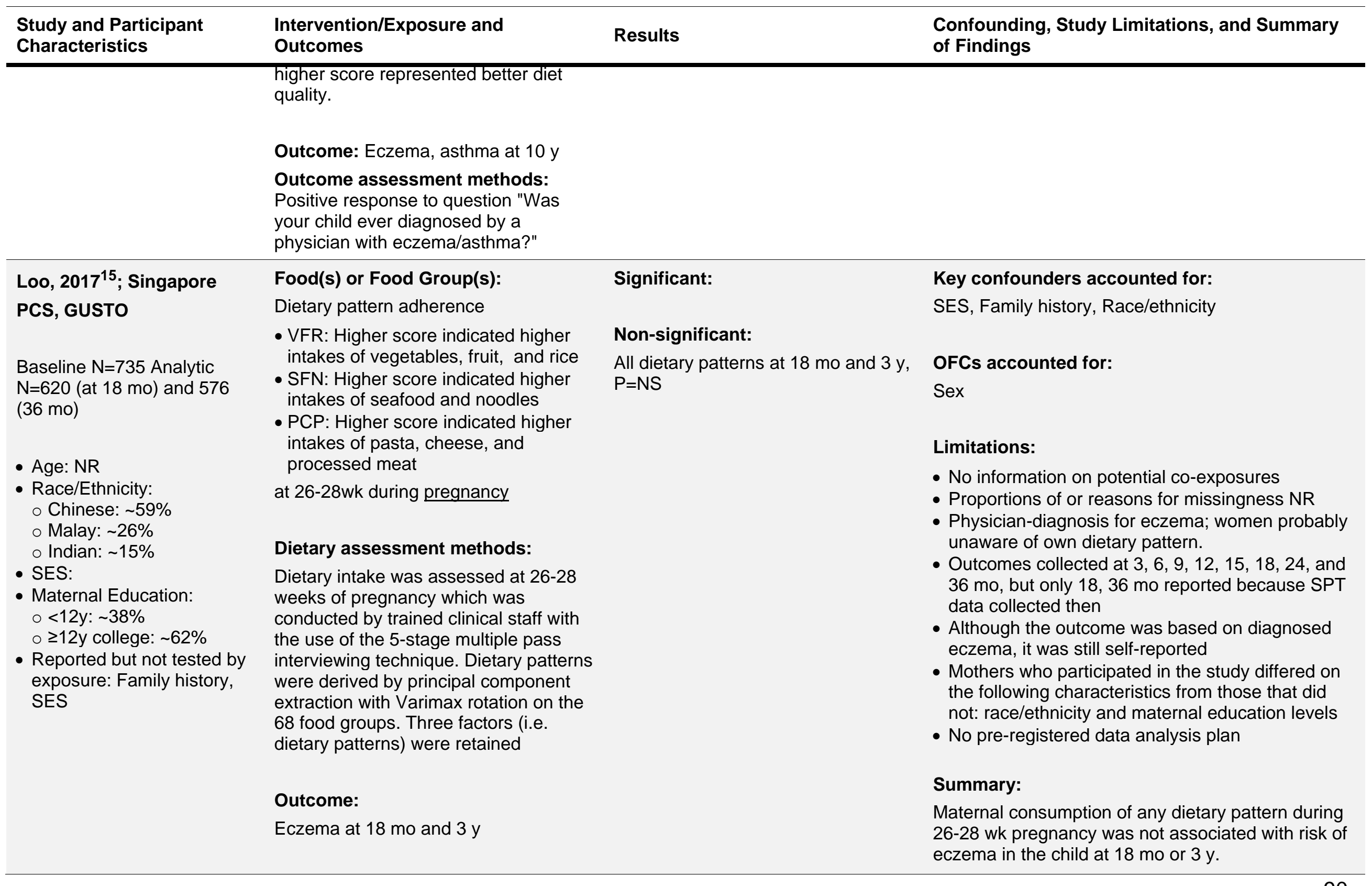




\begin{tabular}{|c|c|c|c|}
\hline $\begin{array}{l}\text { Study and Participant } \\
\text { Characteristics }\end{array}$ & $\begin{array}{l}\text { Intervention/Exposure and } \\
\text { Outcomes }\end{array}$ & Results & $\begin{array}{l}\text { Confounding, Study Limitations, and Summary } \\
\text { of Findings }\end{array}$ \\
\hline & $\begin{array}{l}\text { Outcome assessment methods: } \\
\text { Physician-diagnosed atopic eczema } \\
\text { was based on a positive answer to the } \\
\text { written question: "Has your child ever } \\
\text { been diagnosed with eczema?" }\end{array}$ & & \\
\hline $\begin{array}{l}\text { Jedrychowski, } 2011^{11} \text {; U.S. } \\
\text { \& Poland } \\
\text { PCS } \\
\text { Baseline N=469 Analytic } \\
\text { N=469 } \\
\text { Of } 505 \text { women enrolled, } 469 \\
\text { gave birth to term (>36 wk) } \\
\text { infants. } \\
\text { - Age: } 27.6 \text { y } \\
\text { - Race/Ethnicity: NR } \\
\text { - SES: } \\
\text { ○ Maternal education: 15.7 } \\
\text { y } \\
\text { - Characteristics reported but } \\
\text { no test for differences by } \\
\text { exposure } \\
\text { ○ Smoking (environmental) } \\
\text { ○ Family history } \\
\text { ○ GA } \\
\text { o BW } \\
\text { ○ HMF }\end{array}$ & $\begin{array}{l}\text { Food(s) or Food Group(s): } \\
\text { Total fish intake (g/wk) } \\
\text { - } \leq 90: \mathrm{n}=176 \text { (Ref) } \\
\text {-91-205: } \mathrm{n}=168 \\
\text { - >205: } \mathrm{n}=125 \\
\text { in second and third trimester of } \\
\text { pregnancy } \\
\text { Dietary assessment methods: } \\
\text { Dietary intake assessed during second } \\
\text { and third trimester of pregnancy using } \\
\text { an interviewer-administrated FFQ. In } \\
\text { the course of the food interviews, } \\
\text { detailed information on the eating } \\
\text { frequency of smoked, fried, roasted } \\
\text { and grilled fish servings was also } \\
\text { collected. Maternal fish intake was } \\
\text { categorized as follows: never; less than } \\
\text { once per month; once per week; } 1-2 \\
\text { times per week; } 3-4 \text { times per week, or } \\
\text { every day. To estimate the total amount } \\
\text { of fish eaten per week, it was assumed } \\
\text { that each fish meal averaged } 150 \mathrm{~g} \text {. } \\
\text { Outcome: Atopic Dermatitis: Eczema } \\
\text { at any time during 3-12 mo } \\
\text { Outcome assessment methods: }\end{array}$ & 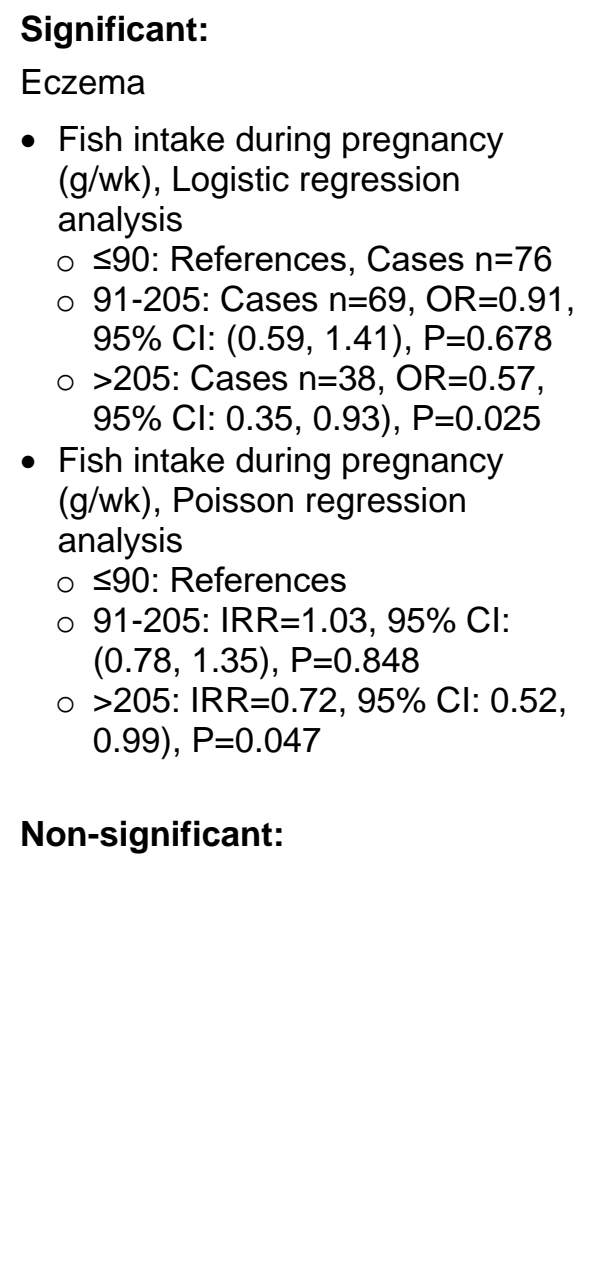 & $\begin{array}{l}\text { Key confounders accounted for: } \\
\text { Age, SES, Smoking, (Family history, only in logistic } \\
\text { regression analysis), HMF, GA } \\
\text { OFCs accounted for: } \\
\text { Sex, Indoor environment } \\
\text { Limitations: } \\
\text { - Outcomes were subjective and were reported by } \\
\text { the participants, who were aware of the exposure } \\
\text { received } \\
\text { - The study reported any eczema, but it is unclear if } \\
\text { eczema at individual timepoints differed (Outcome } \\
\text { was assessed at multiple time points ( } 3,6,9 \text {, and } \\
12 \text { mo). } \\
\text { - The study was conducted in New York City and } \\
\text { Krakow, Poland. It is unclear if same/similar } \\
\text { instruments were used and if the personnel were } \\
\text { trained in a similar way. The comparability of the } \\
\text { tools not specified (assuming that a translated } \\
\text { tool was used in Poland). } \\
\text { - Unclear if the FFQ was validated and the food } \\
\text { items in the FFQ between the two sites were } \\
\text { comparable. } \\
\text { - The sample size and outcome incidence in New } \\
\text { York City and Krakow was not specified. Other } \\
\text { factors that might have influenced exposures and } \\
\text { outcomes between the sites were not stated } \\
\text { clearly. }\end{array}$ \\
\hline
\end{tabular}


Study and Participant

Characteristics

\section{Intervention/Exposure and}

Outcomes

After delivery, a detailed, standardized,

face-to-face interview on the infant's

health was administered to each

mother by a trained interviewer at each

of the study periods 3, 6, 9 and $12 \mathrm{mo}$

after birth. During the interview, a

history of infantile eczema was

recorded if the child experienced dry

skin in combination with itchy rash and

typical localization, which were

confirmed by a physician.

\section{Shaheen, 2009 ${ }^{29}$; U.K.}

\section{PCS, ALSPAC}

Baseline $\mathrm{N}=12,008$ Analytic $\mathrm{N}=9,516$

- Age:

o <25y: $21.4 \%$

० 25-29y: $39.2 \%$

○ $30-34 \mathrm{y}: 29.1 \%$

o $\geq 35 \mathrm{y}: 10.3 \%$

- Race/Ethnicity

○ White: $96.3 \%$

o Non-White: $2.5 \%$

o Unknown: $1.1 \%$

- SES:

- Maternal education:

- <O level: $29.3 \%$

- O level: $34.7 \%$

- A level+: $35.4 \%$

o Housing tenure:

- Owned/mortgaged:

$73.8 \%$

- Council rented: $12.1 \%$

- Non-council rented: $8.0 \%$

\section{Results}

Confounding, Study Limitations, and Summary of Findings

\section{Summary:}

The highest intake of fish, but not moderate intake, during pregnancy was associated with lower odds and cumulative frequency of eczema in children 3$12 \mathrm{mo}$.

\section{Significant:}

\section{Non-significant:}

Atopic dermatitis (Eczema) at 2.5y, $\mathrm{n}=9516$

- Health conscious pattern: High consumption of salad, fruit, fruit juices, rice, pasta, oat/bran based breakfast cereals, fish, pulses, cheese, and non-white bread. Low consumption of white bread.

Traditional pattern: High consumption of potatoes (not chips), vegetables, red meat, and poultry.

- Processed pattern: High consumption of meat pies, sausages, burgers, fried foods, pizza, chips, roast potatoes, white bread, eggs, and baked beans. Low consumption of non-white bread.

- Confectionery pattern: High consumption of chocolate, sweets, biscuits, cakes/buns, puddings, and crisps.

- Vegetarian pattern: High consumption of meat substitutes, pulses, nuts, and herbal tea. Low consumption of poultry and red meat. at 32 wk during pregnancy
- Health conscious: $P=0.08$

- Traditional DP, $\mathrm{P}=\mathrm{NS}$

- Processed DP, $P=N S$

- Confectionary, $\mathrm{P}=\mathrm{NS}$

- Vegetarian, $\mathrm{P}=\mathrm{NS}$

Atopic dermatitis (Eczema) at 7.5y, $\mathrm{n}=7693$

- Health conscious DP, $\mathrm{P}=\mathrm{NS}$

- Traditional DP, $P=N S$

- Processed DP, $P=N S$

- Confectionary, $\mathrm{P}=\mathrm{NS}$

- Vegetarian, $\mathrm{P}=\mathrm{NS}$

\section{Key confounders accounted for:}

Age, Race/ethnicity, SES, Smoking, Family history, GA, BW, HMF, Animals/pets/farming exposure

\section{OFCs accounted for:}

Child sex, Indoor and outdoor environment

\section{Limitations:}

- At least one key confounder was not controlled for

- Authors adjusted for post-exposure variables

- FFQ was not formally validated, but was based on a validated FFQ

- Proportions of missing participants differ substantially across exposures and the analysis is unlikely to have removed the risk of bias arising from the missing data

- Majority of outcomes were subjective and the outcomes were assessed by participants, who

were aware of the exposure received

- No pre-registered data analysis plan

\section{Summary:}




\section{Study and Participant}

Characteristics

- Unknown/other: 6.1\%

o Financial difficulties:

- None: $35.8 \%$

- Some: $38.0 \%$

- Many: $25.7 \%$

- Unknown: 0.5\%

- Characteristics reported

with significant differences

by exposure

○ Smoking

o HMF

- Characteristics reported but

no differences by exposure

o Family history

- GA

○ BW

- Animal/pets/farming

exposure

\section{Intervention/Exposure and}

\section{Outcomes}

Results

\section{Dietary assessment methods:}

43-item FFQ at 32 wk gestation, based on FFQ validated in a British population. 5 dietary patterns were identified in the cohort using PCA: "health conscious", "traditional",

"processed", "vegetarian" and "confectionery". DP scores were expressed in standard deviation units. Each mother was represented in each of these 5 mutually independent scores.

\section{Outcome:}

Atopic dermatitis (Eczema)

\section{Outcome assessment methods:}

The 12 mo prevalence of eczema at 2.5 y and 7.5 y defined by a positive response to the question: "Has your child had an itchy dry skin rash in joints and creases of his/her body (e.g., behind the knees, under the arms) since he/she was 18 months old?"

\section{Bédard, 2020'; U.K.}

PCS, ALSPAC

Baseline $\mathrm{N}=8907$ Analytic

$\mathrm{N}=7705$

- Age: 28.9y

- Race/Ethnicity

\section{Food(s) or Food Group(s):}

Mediterranean Diet (MD) Score adapted for pregnant women.

- MD score 0-3: Lower adherence to a Mediterranean-style diet $(n=3,475)$

- MD score 4-7: Greater adherence to

a Mediterranean-style diet $(n=5,432)$ Score based on the median weekly intake of 6 beneficial food groups
Confounding, Study Limitations, and Summary of Findings

Adherence to dietary patterns during pregnancy, identified using PCA, was not associated with childhood atopic dermatitis at 2.5 and 7.5 y of age.

\section{Significant:}

\section{Non-significant:}

Eczema $(n=7,705)$

- MD score 4-7 vs 0-3: $P=N S$

- Per unit increase: $\mathrm{P}=\mathrm{NS}$

\section{Key confounders accounted for:}

Age, Race/ethnicity, SES, Smoking, Family history, HMF

\section{Limitations:}

- At least one key confounder was not controlled for 


\begin{tabular}{|c|c|c|c|}
\hline $\begin{array}{l}\text { Study and Participant } \\
\text { Characteristics }\end{array}$ & $\begin{array}{l}\text { Intervention/Exposure and } \\
\text { Outcomes }\end{array}$ & Results & $\begin{array}{l}\text { Confounding, Study Limitations, and Summary } \\
\text { of Findings }\end{array}$ \\
\hline 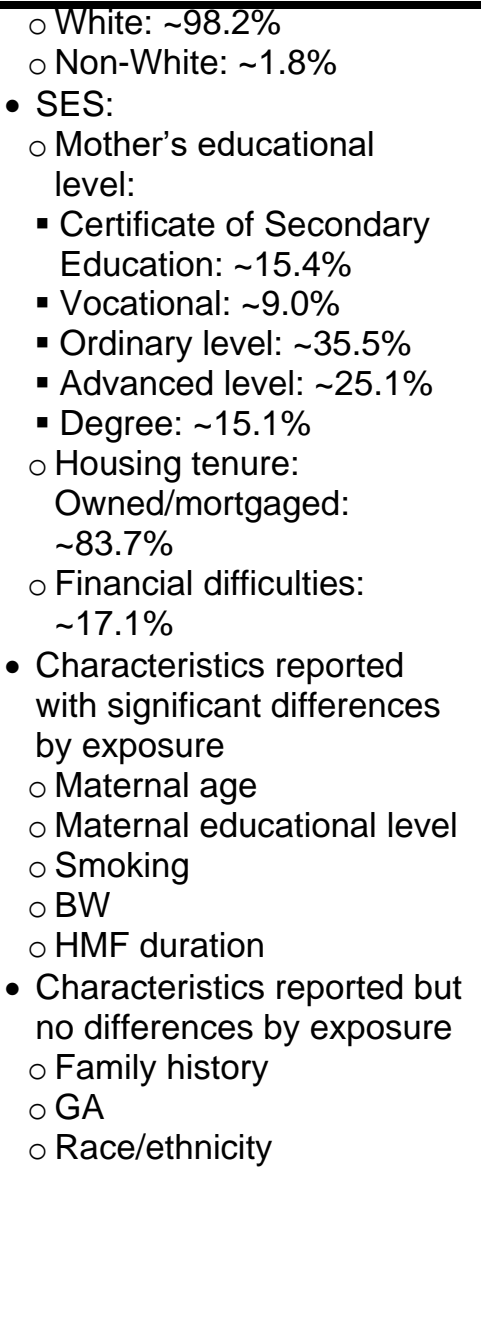 & $\begin{array}{l}\text { (vegetables, legumes, fruits and nuts, } \\
\text { cereal, fish and dairy) and } 1 \text { detrimental } \\
\text { food group (meat). } \\
\text { at } 32 \text { wk during pregnancy } \\
\text { Dietary assessment methods: } \\
\text { 43-item FFQ at } 32 \text { wk gestation, based } \\
\text { on one validated in a British population. } \\
\text { Women whose consumption of } \\
\text { beneficial food groups was above the } \\
\text { median were assigned a value of } 1 \text {, } \\
\text { and those below were assigned a value } \\
\text { of } 0 . \text { For the detrimental food group, } \\
\text { consumption below the median was } \\
\text { assigned a value of } 1 \text {, and above the } \\
\text { median was assigned a value of } 0 \text {. } \\
\text { Food group values were summed } \\
\text { together for a total ranging from } 0 \text { to } 7 \text {, } \\
\text { with a higher score representing } \\
\text { greater adherence to a Mediterranean- } \\
\text { style diet. }\end{array}$ & & $\begin{array}{l}\text { - FFQ was not formally calibrated against other } \\
\text { instruments, but was based on one which has } \\
\text { been validated } \\
\text { - Important co-exposures imbalanced across } \\
\text { groups that were likely to impact the outcome, and } \\
\text { no adjustment techniques used to correct at least } \\
\text { some of those variables } \\
\text { - Outcome measurement was subjective and were } \\
\text { assessed by participants, who were aware of the } \\
\text { exposure received } \\
\text { - Pre-registered data analysis NR } \\
\text { Summary: } \\
\text { Adherence to a Mediterranean-style diet during } \\
\text { pregnancy was not associated with atopic } \\
\text { dermatitis, in the child at } 7.5 \mathrm{y} \text {. }\end{array}$ \\
\hline $\begin{array}{l}\text { Bertelsen, 2014³; Norway } \\
\text { PCS, MoBa }\end{array}$ & $\begin{array}{l}\text { Food(s) or Food Group(s): } \\
\text { - Unexposed: No consumption of } \\
\text { probiotic milk and yogurt, } n=25,572 \\
(63 \%)\end{array}$ & $\begin{array}{l}\text { Significant: } \\
\text { Atopic eczema at } 6 \mathrm{mo} \text { : } \\
\text { aRR: } 0.94,95 \% \mathrm{Cl}: 0.89-0.99\end{array}$ & $\begin{array}{l}\text { Key confounders accounted for: } \\
\text { Age, SES, Smoking, Family history, HMF, Delivery } \\
\text { mode }\end{array}$ \\
\hline
\end{tabular}




\begin{tabular}{|c|c|c|c|}
\hline $\begin{array}{l}\text { Study and Participant } \\
\text { Characteristics }\end{array}$ & $\begin{array}{l}\text { Intervention/Exposure and } \\
\text { Outcomes }\end{array}$ & Results & $\begin{array}{l}\text { Confounding, Study Limitations, and Summary } \\
\text { of Findings }\end{array}$ \\
\hline $\begin{array}{l}\text { Baseline } \mathrm{N}=40,614 \text { Analytic } \\
\mathrm{N}=40614 \\
\text { - Age: } \\
\text { o <20-24y: } 9 \% \\
\text { o } \geq 35 y: 18 \% \\
\text { - Race/Ethnicity: NR } \\
\text { - SES: } \\
\text { o Single: } 3 \% \\
\text { o Maternal Education: } \\
\text { - <High school: } 6 \% \\
\text { - >4y college: } 23 \% \\
\text { - Reported but not tested by } \\
\text { exposure: Smoking, Family } \\
\text { history, BW, GA, Delivery } \\
\text { mode, HMF }\end{array}$ & $\begin{array}{l}\text { - Exposed: Maternal consumption of } \\
\text { probiotic milk and yogurt n=15,042 } \\
\text { (37\%) } \\
\text { during pregnancy (at } 22 \text { wk) } \\
\text { Dietary assessment methods: } \\
\text { Intake of milk-based probiotic products } \\
\text { during pregnancy was recorded in the } \\
\text { FFQ. The women were asked how } \\
\text { often they consumed milk and yogurt, } \\
\text { clearly distinguishing probiotic milk and } \\
\text { yogurt from other milk items. Reported } \\
\text { pregnancy } \\
\text { consumption across all probiotic milk } \\
\text { products was categorized into one } \\
\text { dichotomous variable for any intake } \\
\text { versus no intake and one 3-level } \\
\text { variable based on intake in milliliters } \\
\text { per day categorized as "none," "13.0- } \\
28.3 \text { mL/d," and ">28.4 mL/d" } \\
\text { Outcome assessment methods: } \\
\text { Eczema was classified based on } \\
\text { mothers' responses to a question about } \\
\text { "atopic eczema (childhood eczema)" } \\
\text { asked on both the } 6-\text { and } 18-\text { month } \\
\text { questionnaires. } \\
\text { Current atopic eczema at } 18 \text { mo } \\
\text { Outcome: }\end{array}$ & $\begin{array}{l}\text { Probiotic milk and yogurt } \\
\text { consumption during pregnancy } \\
\text { compared with no consumption } \\
\text { during pregnancy was associated } \\
\text { with a reduced risk of atopic eczema } \\
\text { at } 6 \text { mo } \\
\text { Current atopic eczema at } 18 \mathrm{mo} \text { : } \\
\text { Sensitivity analysis } \\
\text { aRR: } 1.08,95 \% \mathrm{Cl}: 1.01,1.15 \\
\text { Sensitivity analysis of maternal } \\
\text { intake ONLY (not child) showed } \\
\text { higher risk among exposed group } \\
\text { Non-Significant: } \\
\text { Atopic eczema at } 6 \mathrm{mo}, \mathrm{P}=\mathrm{NS} \\
\text { Current atopic eczema at } 18 \mathrm{mo}, \\
\mathrm{P}=\mathrm{NS} \\
\text { - Sensitivity analysis of maternal } \\
\text { AND child intake, } \mathrm{P}=\mathrm{NS} \\
\text { - Densitivity analyses, } \mathrm{P}=\mathrm{NS} \\
\text { o Sex }\end{array}$ & $\begin{array}{l}\text { OFCs accounted for: } \\
\text { Child sex } \\
\text { Limitations: } \\
\text { - Based on the exposure data availability, only a } \\
\text { sub-set of women from MoBa were included in this } \\
\text { study. Mothers who participated in this study were } \\
\text { different than the rest, on the following } \\
\text { characteristics: education, smoking and parity } \\
\text { - Outcome measurement was subjective and were } \\
\text { assessed by participants, who were aware of the } \\
\text { exposure received } \\
\text { Summary: } \\
\text { Maternal consumption of probiotic milk and yogurt } \\
\text { during pregnancy was associated with lower risk of } \\
\text { atopic eczema in the child at } 6 \text { mo, but not with } \\
\text { current atopic eczema at } 18 \text { mo. } \\
\text { In sensitivity analysis, maternal intake (when the } \\
\text { infant received no probiotic milk) was associated } \\
\text { with higher risk of current atopic eczema in the child } \\
\text { at } 18 \text { mo. } \\
\text { All other comparisons and sensitivity analyses } \\
\text { revealed no association between maternal intake } \\
\text { and child atopic eczema at } 6 \text { mo or } 18 \text { mo. }\end{array}$ \\
\hline
\end{tabular}




\begin{tabular}{|c|c|c|c|}
\hline $\begin{array}{l}\text { Study and Participant } \\
\text { Characteristics }\end{array}$ & $\begin{array}{l}\text { Intervention/Exposure and } \\
\text { Outcomes }\end{array}$ & Results & $\begin{array}{l}\text { Confounding, Study Limitations, and Summary } \\
\text { of Findings }\end{array}$ \\
\hline $\begin{array}{l}\text { Bunyavanich, 2014 }{ }^{4} \text {; U.S. } \\
\text { PCS, Project Viva } \\
\text { Baseline N=2128 Analytic } \\
\text { N=1277 } \\
\text { - Age: } 32.3 \text { (from other } \\
\text { Project Viva data) } \\
\text { - Race/Ethnicity: White: } 69 \% \\
\text { - SES: } \\
\text { o Maternal Education: } \\
\text { ocollege graduate: } 69.3 \% \\
\text { o Household income } \geq \$ 70 \mathrm{~K} \text { : } \\
63.0 \% \\
\text { - Reported but not tested by } \\
\text { exposure: Family history }\end{array}$ & $\begin{array}{l}\text { Food(s) or Food Group(s): } \\
\text { Maternal consumption of major food } \\
\text { allergens (servings/d z scores) } \\
\text { - Peanut: } \\
\circ 1^{\text {st }} \text { trimester: } 0.34 \pm 0.44 \\
\circ 2^{\text {nd }} \text { trimester: } 0.36 \pm 0.43 \\
\text { - Milk: } \\
\circ 1^{\text {st }} \text { trimester: } 1.16 \pm 1.04 \\
\circ 2^{\text {nd }} \text { trimester: } 1.50 \pm 1.82 \\
\text { - Wheat: } \\
\circ 1^{\text {st }} \text { trimester: } 2.65 \pm 1.48 \\
\circ 2^{\text {nd }} \text { trimester: } 2.69 \pm 1.44 \\
\text { - Egg: } \\
\circ 1^{\text {st }} \text { trimester: } 0.32 \pm 0.30 \\
\circ 2^{\text {nd }} \text { trimester: } 0.33 \pm 0.30 \\
\text { - Soy: } \\
\circ 1^{\text {st }} \text { trimester: } 0.08 \pm 0.27 \\
\circ 2^{\text {nd }} \text { trimester: } 0.08 \pm 0.28 \\
\text { at } 10 \text { and } 26-28 \text { wk during pregnancy } \\
\text { Dietary assessment methods: } \\
\text { Maternal dietary assessments at the } \\
\text { first and second trimester visits were } \\
\text { based on a validated } 166 \text {-item semi- } \\
\text { quantitative FFQ modified for } \\
\text { pregnancy. The total servings per day } \\
\text { of each major food allergen (peanut, } \\
\text { milk, wheat, egg, and soy) were } \\
\text { calculated by summing the servings per } \\
\text { day of the foods on the FFQ containing } \\
\text { these respective food allergens. }\end{array}$ & $\begin{array}{l}\text { Stratification: Parental atopy } \\
\text { Maternal 1st trimester milk intake } \\
\text { (per } z \text {-score): } \\
\text { aOR=0.64, } 95 \% \mathrm{Cl} \text { : }(0.44,0.96) \\
\text { Non-significant: } \\
\text { Maternal } 1 \mathrm{st} \text { trimester intake of any } \\
\text { food allergens, P=NS } \\
\text { Maternal 2nd trimester intake of } \\
\text { peanut, milk, egg, or soy, P=NS } \\
\text { Stratification by parental atopy } \\
\text { showed no association between } \\
\text { intake of any food allergens during } \\
\text { 1st or 2nd trimester (except 1st } \\
\text { trimester milk with parental atopy). }\end{array}$ & $\begin{array}{l}\text { Key confounders accounted for: } \\
\text { (Race/Ethnicity adjusted in a secondary model), } \\
\text { SES, Family history, HMF } \\
\text { OFCs accounted for: } \\
\text { Child sex } \\
\text { Limitations: } \\
\text { - Critical co-exposures NR } \\
\text { - Mothers who participated in the study were } \\
\text { different than those who were lost to follow-up on } \\
\text { the following characteristics: maternal race, } \\
\text { college education, income, parental atopy } \\
\text { - Proportions of and reasons for missingness NR by } \\
\text { exposure } \\
\text { - Multiple exposure outcome comparisons were } \\
\text { assessed without using an appropriate p-value } \\
\text { correction } \\
\text { - Self-reported exposure and outcome (for clinical } \\
\text { symptoms) } \\
\text { - Pre-registered data analysis plan NR } \\
\text { Summary: } \\
\text { Higher maternal consumption of wheat during the } \\
2 \text { nd trimester of pregnancy was associated with } \\
\text { reduced risk of atopic dermatitis in the child at } 7.9 y \text { y. }\end{array}$ \\
\hline & $\begin{array}{l}\text { Outcome: } \\
\text { Atopic dermatitis at } \sim 7.9 \mathrm{y}\end{array}$ & & \\
\hline
\end{tabular}


Study and Participant
Intervention/Exposure and Outcomes
Results

Confounding, Study Limitations, and Summary of Findings

\section{Outcome assessment methods:}

Current atopic dermatitis was defined as positive if a mother reported at the mid-childhood visit that her child ever had doctor-diagnosed eczema plus an itchy rash in the folds of the elbows, behind the knees, in front of the ankles, under the buttocks, or around the neck, ears, or eyes in the past 12 months that did not go completely away for at least $6 \mathrm{mo}$.

Ever atopic dermatitis were defined as positive if a mother reported a doctor's diagnosis of each respective condition in the child in any questionnaire since birth.

\section{Willers, $2007^{31}$; U.K. PCS}

Baseline $\mathrm{N}=1751$ Analytic $\mathrm{N}=1212$

Of 1751 women who completed FFQ during pregnancy, 1212 completed follow-up when children were $5 y$.

- Age at recruitment: $29.9 y$, $95 \% \mathrm{Cl}:(29.6,30.2)$

- Race/Ethnicity: NR

- SES:

\section{Food(s) or Food Group(s):}

Apple consumption by tertile

- T1: 0-1/wk, $\mathrm{n}=398$

- T2: $1-4 / w k, n=427$

- T3: $>4 / w k, n=384$

at $\sim 32$ wk during pregnancy

Fish consumption

- Never, $n=107$

- $<1 /$ wk, $n=255$

$\bullet \geq 1 / \mathrm{wk}, \mathrm{n}=831$

at $\sim 32$ wk during pregnancy

Oily fish consumption

- Never, $n=629$

\section{Significant:}

Doctor-confirmed eczema

- Fish intake, Cases $n=380, P$ for trend $=0.008$

o Never: Ref

$0<1 /$ wk: aOR=0.79, 95\% Cl: $(0.47$, 1.32) $\circ \geq 1 /$ wk: aOR $=0.57,95 \% \mathrm{Cl}:(0.35$, $0.92)$

Current eczema medication

- Fish intake, Cases n=191, P for trend $=0.028$

o Never: Ref

$0<1 /$ wk: aOR=0.88, 95\% Cl: $(0.46$, 1.67)

\section{Key confounders accounted for:}

Age, SES, Smoking (Mother, Household), Family history, BW, HMF

\section{OFCs accounted for:}

Child sex

\section{Limitations:}

- Mothers who participated in the study were of higher SES and had slightly higher consumption of fruits, green leafy vegetables, whole grain products and fish. They also had fewer respiratory symptoms.

- Multiple exposure outcome comparisons were assessed without using an appropriate $p$-value correction. 


\begin{tabular}{|c|c|c|c|}
\hline $\begin{array}{l}\text { Study and Participant } \\
\text { Characteristics }\end{array}$ & $\begin{array}{l}\text { Intervention/Exposure and } \\
\text { Outcomes }\end{array}$ & Results & $\begin{array}{l}\text { Confounding, Study Limitations, and Summary } \\
\text { of Findings }\end{array}$ \\
\hline $\begin{array}{l}\circ \text { Partner from non-manual } \\
\text { social class: } 62.7 \% \\
\circ \text { Age left full time } \\
\text { education: Median=18.5y, } \\
\text { IQR=(16.0, 21.0) } \\
\text { - Characteristics reported but } \\
\text { no test for differences by } \\
\text { exposure } \\
\circ \text { Smoking } \\
\text { ○ Family history } \\
\circ \text { BW } \\
\circ \text { HMF (Ever) }\end{array}$ & $\begin{array}{l}\bullet<1 / \text { wk, } \mathrm{n}=414 \\
\bullet \geq 1 / \text { wk, } \mathrm{n}=161 \\
\text { at } ~ 32 \text { wk during pregnancy } \\
\text { Dietary assessment methods: } \\
\text { Semi-quantitative, } 150 \text {-item } \mathrm{FFQ}, \\
\text { divided into } 20 \text { food groups to capture } \\
\text { intake over the previous } 2-3 \text { mo. } \\
\text { Responses were categorized as rarely } \\
\text { or never, } 1-2 \text { times/mo, and separate } \\
\text { categories for } 1-7 \text { d/wk. Food amounts } \\
\text { were recorded as } 1 \text { to } \geq 5 \text { measures per } \\
\text { day. The number of measures/d was } \\
\text { multiplied by the number of d/wk to } \\
\text { obtain the total measures/wk. } \\
\text { The food groups of interest in this study } \\
\text { were total fruit, citrus/kiwi fruit, apples, } \\
\text { total vegetables, green leafy } \\
\text { vegetables, pure fruit juice, whole grain } \\
\text { products, total fish, total oily fish, total } \\
\text { fat from dairy products and exclusive } \\
\text { butter versus margarine/low fat spread } \\
\text { used as spread. The total number of } \\
\text { measures per week was divided into } \\
\text { tertiles for total fruit, citrus/kiwi fruit, } \\
\text { apples, total vegetables, green leafy } \\
\text { vegetables, pure fruit juice, whole grain } \\
\text { products and into the categories never, } \\
\text { less than once a week, and once or } \\
\text { more a week for total fish and total oily } \\
\text { fish. To facilitate extrapolation to the } \\
\text { general population, subdivisions of food } \\
\text { intakes into tertiles were derived from } \\
\text { all of the women completing the FFQ } \\
\text { and not merely those responding at } 5 \\
\text { years. }\end{array}$ & $\begin{array}{l}0 \geq 1 / \mathrm{wk} \text { : aOR=0.58, } 95 \% \mathrm{Cl} \text { : }(0.32 \text {, } \\
1.06) \\
\text { Non-significant: } \\
\text { Ever had eczema } \\
\text { - Fish intake, Cases } \mathrm{n}=406, \mathrm{P} \text { for } \\
\text { trend=0.050 } \\
\text { o Never: Ref } \\
0<1 / \mathrm{wk} \text { : aOR=0.91, } 95 \% \mathrm{Cl} \text { : }(0.54 \text {, } \\
1.53) \\
0 \geq 1 / \mathrm{wk} \text { : aOR=0.68, } 95 \% \mathrm{Cl} \text { : }(0.43 \text {, } \\
1.10) \\
\text { There were no consistent linear } \\
\text { associations between maternal } \\
\text { intake of total fruit, citrus/kiwi fruit, } \\
\text { total vegetables, green leafy } \\
\text { vegetables, fruit juice, whole grain } \\
\text { products, fat from dairy products or } \\
\text { butter versus margarine/low fat } \\
\text { spread use and respiratory or atopic } \\
\text { outcomes in children at } 5 \mathrm{y}, \text { nor were } \\
\text { there consistent associations } \\
\text { between maternal intake of food } \\
\text { groups and spirometry, atopic } \\
\text { sensitization, bronchodilator } \\
\text { response or exhaled nitric oxide. }\end{array}$ & $\begin{array}{l}\text { Summary: } \\
\text { Higher maternal fish intake was associated with } \\
\text { lower odds of doctor-confirmed eczema and odds of } \\
\text { current eczema medication in the child at } 5 y \text {, but } \\
\text { only marginally significantly associated with lower } \\
\text { odds of ever having had eczema. } \\
\text { Higher maternal oily fish intake was associated with } \\
\text { lower odds of doctor-confirmed hay fever in the child } \\
\text { at age } 5 \mathrm{y} \text {, but was not associated with current hay } \\
\text { fever medication or ever having had hay fever. } \\
\text { No other foods or food group(s) measured were } \\
\text { associated with respiratory or atopic outcomes. }\end{array}$ \\
\hline
\end{tabular}


Study and Participant

Characteristics
Intervention/Exposure and

Outcomes
Results

Confounding, Study Limitations, and Summary

of Findings
Outcome: Asthma, Allergic dermatitis (eczema), Allergic rhinitis (hay fever)

at $5 \mathrm{y}$

Outcome assessment methods:

Based on ISAAC core questions on symptoms of asthma, allergic rhinitis and atopic eczema, including the

questions: "Has your child ever

suffered from asthma?", "Has this been

confirmed by a doctor?" and "Has your

child received treatment for asthma in

the past 12 months?" with similar

questions enquired about eczema and

hay fever.

\section{Pregnancy and Lactation}

\section{Randomized Controlled Trials}

Fukushima, $1997^{8}$; Japan RCT

Baseline $\mathrm{N}=350$ Analytic $\mathrm{N}=283$

- Age: NR

- Race/Ethnicity: 100\% from

Japan

- SES: NR

- Significant differences by exposure (by design): HMF, CFB

- No differences by exposure: Smoking, Family history, BW, Pets

\section{Food(s) or Food Group(s):}

- CD: Mothers instructed to consume $>200 \mathrm{~mL} / \mathrm{d}$ cow milk, $\mathrm{n}=140$ randomized, $n=127$ analyzed

- MD: Mothers instructed to consume $>200 \mathrm{~mL} / \mathrm{d}$ a casein-free, hypoallergenic formula and to avoid cow milk and reduce consumption of cow milk products, $n=140$ randomized, $n=102$ analyzed

- AF: Mothers instructed to consume $>200 \mathrm{~mL} / \mathrm{d}$ a casein-free,

hypoallergenic formula and to avoid cow milk and reduce consumption of cow milk products, $\mathrm{n}=70$ randomized, $\mathrm{n}=54$ analyzed (Not randomized, no relevant comparisons included,

\section{Significant:}

Eczema prevalence at $6 \mathrm{mo}, \mathrm{P}<0.05$

- MD (Ref): 13\%

- CD: $\sim 25 \%$

Eczema prevalence at $12 \mathrm{mo}, \mathrm{P}<0.01 \quad$ None

- MD (Ref): $\sim 11 \%$

- CD: $\sim 26 \%$

aOR=3.02, 95\% Cl: $(1.44,6.33)$

Eczema prevalence at $18 \mathrm{mo}, \mathrm{P}<0.01$

- MD (Ref): $\sim 12 \%$

- CD: $\sim 27 \%$

Eczema prevalence at $24 \mathrm{mo}, \mathrm{P}<0.05$

- MD (Ref): 12\%

\section{Key confounders accounted for:}

Smoking, Family history, (CFB for aOR), Pets

\section{OFCs accounted for:}

\section{Limitations:}

- Randomization process NR

- MD still consumed cow milk products, but had lower intake than CD

- Attrition higher in MD group because women excluded from analysis if they consumed cow milk

- Proportions of missing data seem to differ, reasons for missingness NR 


\section{Study and Participant}

Characteristics

$\sim 76 \%$ had a positive history of allergy (i.e. both parents or one parents or sibling only had history of allergies)

\section{Intervention/Exposure and}

\section{Outcomes}

except as co-variates in logistic

regressions)

- Also supplemented with 1000 mg/d $\mathrm{Ca}$

during late pregnancy through end of lactation up to 6 mo postpartum

From birth to $6 \mathrm{mo}$, the infants in the $M D$ and $C D$ groups were exclusively HMF or mixed-fed with breast milk and casein-free, hypoallergenic formula when breast milk was insufficient. The infants in the AF group were mixed-fed with breast milk and a formula with similar whey:casein ratio as breast milk for the corresponding $6 \mathrm{mo}$. Infants who were fed breast milk exclusively from birth to $4 \mathrm{mo}$ were excluded from the AF group.

\section{Dietary assessment methods:}

Daily food diary from late pregnancy until 6 mo postpartum, recording the amount of casein-free, hypoallergenic formula, cow milk, cow milk products, eggs, meat, and soy products consumed.

\section{Outcomes:}

Atopic Dermatitis: Eczema at $6,12,18$, and $24 \mathrm{mo}$

Overall allergies (eczema, asthma, and/or allergic rhinitis)

at $24 \mathrm{mo}$
Confounding, Study Limitations, and Summary

of Findings

-CD: $\sim 23 \%$

$\mathrm{aOR}=2.12,95 \% \mathrm{Cl}:(1.04,4.31)$

Note, CD had significantly higher odds of overall allergies vs MD at $12 \mathrm{mo}$, but due to inclusion of asthma these results to not meet NESR criteria

\section{Non-significant:}

Overall allergies at $24 \mathrm{mo}, \mathrm{P}=\mathrm{NS}$ - $\mathrm{CD}$ : $\mathrm{aOR}=1.75,95 \% \mathrm{Cl}:(0.94$ 3.25)

-MD: (Ref)

Note, odds of overall allergies NS at 6 and $18 \mathrm{mo}$, but due to inclusion of asthma these results to not meet NESR criteria
- $\quad$ Participants were aware of exposure status and outcomes were self-reported

- $\quad$ Eczema logistic regressions only reported at 12 and 24 mo, but data were collected at all 4 time points

- Pre-registered data analysis plan NR

\section{Summary:}

Consuming diets replacing cow milk with casein-free hypoallergenic formula and reduced consumption of cow milk products from late pregnancy through lactation resulted in a lower risk of eczema in the child at $6,12,18$, and 24 months. 
Study and Participant
Intervention/Exposure and

Outcomes
Results

Confounding, Study Limitations, and Summary of Findings
Outcome assessment methods:

Self-reported questionnaire. Specific allergies defined when symptoms chronically lasted for more than a few weeks.

Atopic eczema defined as skin areas of scaly erythematous and itchy rash primarily involving the facial area, area behind the ears and flexural folds were present.

\section{Lovegrove, $1994^{16}$; U.K. RCT}

Baseline $\mathrm{N}=44$ Analytic $\mathrm{N}=38$

- Age: 31y

- Race/Ethnicity: NR

- SES: $100 \%$ have partners

- No differences by exposure: Family history (randomized groups only), HMF

Women or her partner were atopic, defined as having eczema, asthma, hayfever and atopic dermatitis.

Anecdotal symptoms were not accepted and only those who suffered from allergies that were clinically diagnosed were accepted as atopic.

\section{Food(s) or Food Group(s):}

- Atopic: Mothers with diagnosed allergy or partner with diagnosed allergy encouraged to follow standard diets. Consumed $\geq 500 \mathrm{ml}$ cows' milk daily, $n=14$

- Non-atopic: No allergy in mothers or partners. Mothers encouraged to follow standard diets (Not

randomized, no relevant comparisons included), $n=12$

- Atopic-diet: Mothers with diagnosed allergy or partner with diagnosed allergy instructed to avoid all milk and dairy products. As a milk alternative, a hypoallergenic, complete infant formula, was given to the mothers to consume as required, $n=12$

o Also supplemented with 1000 mg/d $\mathrm{Ca}$

from 36 wk gestation through lactation

All groups encouraged to practice

\section{Significant:}

Eczema at $18 \mathrm{mo}$

- Incidence, $\mathrm{P}<0.04$

- Atopic $(n=7)>$ Atopic-diet $(n=4)$,

\section{Non-significant:}

Eczema at 6 mo

- Incidence, $P=N S$

- Severity, $P=N S$

Eczema at $12 \mathrm{mo}$

- Incidence, $\mathrm{P}=\mathrm{NS}$

- Severity, $P=N S$

Eczema at $18 \mathrm{mo}$ - Severity, $P=N S$

\section{Key confounders accounted for:}

Age, SES, Family history, HMF

\section{OFCs accounted for:}

Sex

\section{Limitations:}

- Randomization process NR

- One pair of twins in non-atopic group, but this appears to be accounted for by the analyses first formula exposure Atopic (1mo) earlier than Atopic-diet (5mo), $\mathrm{P}<0.005$

- Adherence appeared to be reasonable among those who completed, but $14 \%$ attrition is not explained and no appropriate analysis performed

- Reasons for and proportions of missingness NR
- No differences in duration of HMF, but timing of

- Pre-registered data analysis NR

\section{Summary:}


Study and Participant

Characteristics
Intervention/Exposure and

Outcomes

EHMF for 6 mo. Solid feeding

discouraged until $3 \mathrm{mo}$ and started

with baby rice and vegetables.

Provision of cows' milk to the infant was discouraged until $\geq 6 \mathrm{mo}$. Other

weaning practices performed at

mother's discretion.

\section{Dietary assessment methods:}

$7 \mathrm{~d}$ weighed food inventory. Women following the restricted diet asked to record any dietary non-adherence.

\section{Outcome:}

Atopic Dermatitis: Eczema

at 6,12 , and $18 \mathrm{mo}$

\section{Outcome assessment methods:}

Blinded doctor diagnosis

Severity of eczema based on area and degree of skin irritation scaled from 1 to

3 , with 1 indicating least severe allergy

\section{Results}

Confounding, Study Limitations, and Summary of Findings

Among mothers who were atopic or had atopic partners, consuming diets free of milk and dairy products from 36 weeks gestation through lactation resulted in a lower incidence of eczema in the child at 18 months, but not at 6 months or 12 months. There was no effect on severity of eczema in the child.

\section{Zeiger, $1989^{35}$; U.S.}

RCT

Baseline $\mathrm{N}=379$ Analytic $\mathrm{N}=288$

Power analysis: Yes

Of these families, 14 in each group were found to be not

\section{Food(s) or Food Group(s):}

- Control: Mothers encouraged to follow standard diets during $3^{\text {rd }}$ trimester of pregnancy and lactation, $\mathrm{n}=212$

- Cow milk-based whey infant formula provided for supplementation or weaning through $12 \mathrm{mo}$ postpartum. CFB encouraged: no solids $<4 \mathrm{mo}$, cereal at $4 \mathrm{mo}$, followed by vegetables, fruits and egg yolks at

\section{Significant:}

\section{Non-significant:}

Period prevalence of atopic dermatitis, $\mathrm{P}=\mathrm{NS}$ at each time point at 4,12 , and $24 \mathrm{mo}$

- Atopic dermatitis (at $12 \mathrm{mo}$, $\mathrm{P}=0.052)$
Key confounders accounted for: Age

Race/Ethnicity, SES, Smoking, Family history, HMF, CFB, Pets

\section{Limitations:}

- More women in the prophylactic-treated group withdrew before delivery because of the protocol's dietary restrictions. As a result, halfway through recruitment, randomization was changed from 40:60 (treatment: control) to 50:50 with a new computerized list of random numbers 


\begin{tabular}{|c|c|c|c|}
\hline $\begin{array}{l}\text { Study and Participant } \\
\text { Characteristics }\end{array}$ & $\begin{array}{l}\text { Intervention/Exposure and } \\
\text { Outcomes }\end{array}$ & Results & $\begin{array}{l}\text { Confounding, Study Limitations, and Summary } \\
\text { of Findings }\end{array}$ \\
\hline 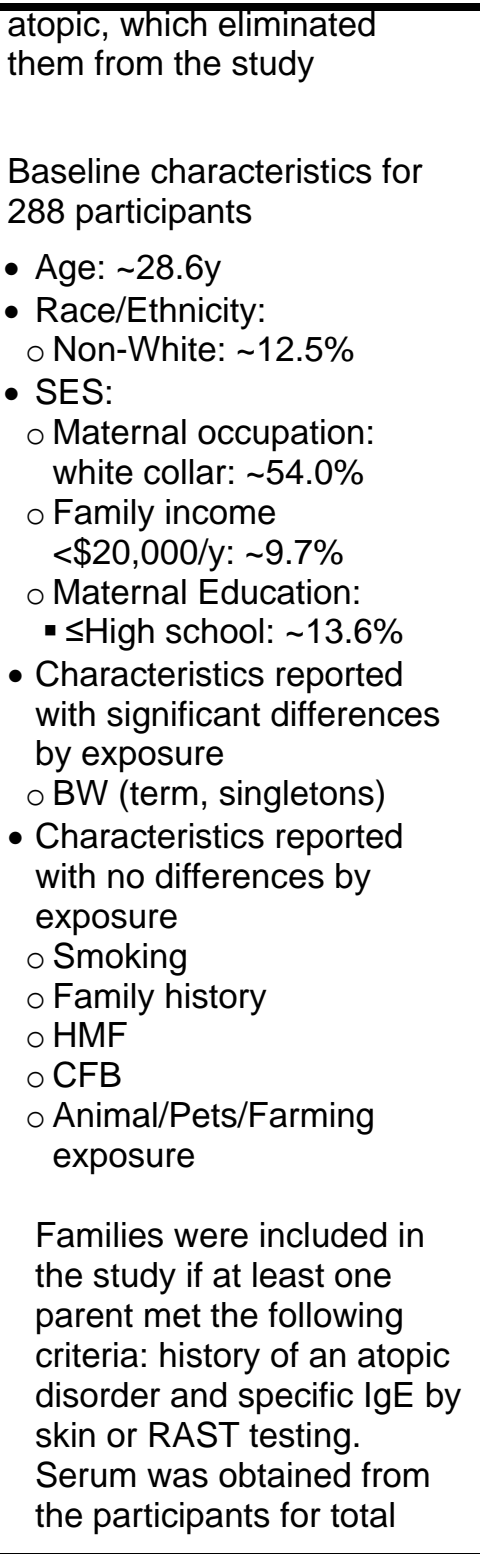 & $\begin{array}{l}\text { 6mo, meats at } 8 \text { mo, and whole cow } \\
\text { milk and egg whites at } 12 \text { mo. } \\
\text { - Prophylaxis: Instructed to avoid totally } \\
\text { all milk (dairy), egg, and peanut } \\
\text { products, avoid concentrated soy } \\
\text { foods (i.e., tofu), } \leq 2 \text { servings/d wheat, } \\
\text { with other grains to fulfill cereal and } \\
\text { starch requirements during } 3^{\text {rd }} \\
\text { trimester of pregnancy and lactation, } \\
n=167 \\
\text { In addition to prenatal vitamins, } \\
\text { the maternal diet was } \\
\text { supplemented with a total of } 1500 \\
\text { mg/d Ca. } \\
\text { A casein hydrolysate infant } \\
\text { formula with low sensitization } \\
\text { potential provided for } \\
\text { supplementation or weaning } \\
\text { through } 12 \text { mo postpartum. CFB } \\
\text { encouraged: no solids }<6 \text { mo, non- } \\
\text { legume vegetables, followed by } \\
\text { rice cereal at } 7 \text { mo, meats at } 8 \text { mo, } \\
\text { non-citrus fruits and juices at } 9 \mathrm{mo}, \\
\text { and cow milk at } 12 \text { mo. Wheat, } \\
\text { soy, corn, and citrus introduced } \\
\text { thereafter at monthly intervals, } \\
\text { followed by egg at } 24 \text { mo and } \\
\text { peanuts and fish at } 36 \text { mo. } \\
\text { - Both groups encouraged to feed } \\
\text { human milk for } \geq 4-6 \text { mo } \\
\text { Dietary assessment methods: } \\
\text { Women were randomly assigned to } \\
\text { groups. In addition to instructions } \\
\text { described above, women in prophylaxis } \\
\text { group attended a dietary class was } \\
\text { held before the } 3 r d \text { trimester by a } \\
\text { licensed dietitian to provide detailed }\end{array}$ & $\begin{array}{l}\text { Cumulative prevalence of atopic } \\
\text { dermatitis, } \mathrm{P}=\mathrm{NS} \text { at each time point } \\
\text { at } 4,12 \text {, and } 24 \mathrm{mo} \\
\text { - Atopic dermatitis (at } 12 \mathrm{mo} \text {, } \\
\mathrm{P}=0.059 \text { ) }\end{array}$ & $\begin{array}{l}\text { - Rate of drop-out was significantly different in the } \\
\text { prophylaxis vs. control groups ( } \mathrm{P}<0.0001) \\
\text { - Significantly more non-White fathers in } \\
\text { prophylaxis group, and trend to more mothers with } \\
\text { low education in control group } \\
\text { - } 6 \text { pairs of twins omitted from control group after } \\
\text { randomization } \\
\text { - BW } 2.9 \% \text { lower in term, singletons in prophylactic } \\
\text { group ( } \mathrm{P}=0.044) \text {; Overall, BW similar between } \\
\text { groups } \\
\text { - Smoking during postpartum was significantly } \\
\text { different between the prophylactic and control } \\
\text { groups } \\
\text { - Some missing data, but power calculation } \\
\text { suggests the analytic } N \text { is sufficient to test } \\
\text { hypotheses. } \\
\text { - Physician making diagnosis was aware of } \\
\text { exposure status; outcomes required both lab tests } \\
\text { and observations at multiple time points, with } \\
\text { similar results for "probable" and "definite" } \\
\text { diagnoses. } \\
\text { - No pre-registered data analysis plan. } \\
\text { Summary: } \\
\text { Maternal avoidance of milk (dairy), egg, and peanut } \\
\text { products, concentrated soy foods, and limited intake } \\
\text { of wheat during the } 3^{\text {rd }} \text { trimester and lactation did } \\
\text { not impact the incidence of atopic dermatitis in the } \\
\text { child. }\end{array}$ \\
\hline
\end{tabular}




\section{Study and Participant}

Characteristics

and specific lgE

determinations. Participant

fathers were skin tested to

inhalant antigens at the

intake session. Mothers

were skin tested to foods

and inhalants $4 \mathrm{mo}$

postpartum.

\section{Intervention/Exposure and}

Outcomes

instructions on the maternal and infant

diets, food lists, recipes, and product

sources. Adherence to the dietary

regimen was ascertained in part by

maternal self-report and daily diaries.

For both groups: $0.25 \mathrm{mg} / \mathrm{d}$ Tri-Vi-Flor given to infants according to their pediatrician's preference. Foods causing documented IgE sensitization were removed from the infant's diet until sensitization had waned or were tolerated on double-blind challenge. Parents received intensive education on reducing environmental allergens and tobacco smoke from their homes.

Outcome: Atopic dermatitis, Allergic rhinitis, Food allergy

\section{at $1,4,8$, and $12 \mathrm{mo}$}

Outcome assessment methods:

Atopic dermatitis was defined as an eczematous eruption that met 3 (probable) or 4 (definite) of the following criteria: pruritus, typical morphology and distribution, a tendency toward chronicity or recurrence, and concurrent specific $\lg \mathrm{E}$ at the time the rash was present
Results

Confounding, Study Limitations, and Summary of Findings

Food(s) or Food Group(s):

- Control: Mothers encouraged to follow standard diets during 3rd trimester of pregnancy and lactation

- Prophylaxis: Avoided all milk (dairy), egg, and peanut products,

\section{Significant:}

\section{Non-Significant:}

Period prevalence at 3 and $4 \mathrm{y}$ :

\section{Key confounders accounted for:}

Age, Race/Ethnicity, SES, Smoking, Family history, HMF, CFB, Pets

\section{Limitations:}




\begin{tabular}{|c|c|c|c|}
\hline $\begin{array}{l}\text { Study and Participant } \\
\text { Characteristics }\end{array}$ & $\begin{array}{l}\text { Intervention/Exposure and } \\
\text { Outcomes }\end{array}$ & Results & $\begin{array}{l}\text { Confounding, Study Limitations, and Summary } \\
\text { of Findings }\end{array}$ \\
\hline $\begin{array}{l}\text { Baseline } N=379 \text { Analytic } \\
N=242 \text { (at } 3 \text { y) and } 225 \text { (at } \\
4 y \text { ) } \\
\text { See Zeiger, } 1989^{35}\end{array}$ & $\begin{array}{l}\text { concentrated soy foods (i.e., tofu), } \leq 2 \\
\text { servings/d wheat during 3rd trimester } \\
\text { of pregnancy and lactation } \\
\text { o Prenatal vitamins plus supplemented } \\
\text { with a total of } 1500 \mathrm{mg} / \mathrm{d} \mathrm{Ca} \\
\text { Both groups encouraged to feed } \\
\text { human milk for } \geq 4-6 \text { mo } \\
\text { Outcome: } \\
\text { Atopic Dermatitis at } 3 \text { y and } 4 y \\
\text { Period prevalence: Defined as the } \\
\text { proportion of subjects currently } \\
\text { evidencing a measured parameter } \\
\text { Cumulative prevalence: Defined as the } \\
\text { proportion of subject evidencing the } \\
\text { measured parameter at any past or } \\
\text { current time } \\
\text { Prophylactic infants used casein } \\
\text { hydrolysate (Nutramigen) for } \\
\text { supplementation or weaning, and } \\
\text { avoided solid foods for } 6 \text { mo; cow milk, } \\
\text { corn, soy, citrus, and wheat, for } 12 \text { mo; } \\
\text { and egg, peanut, and fish, for } 24 \text { mo. } \\
\text { Infants in the control groups Enfamil, a } \\
\text { cow milk-based whey infant formula. } \\
\text { Solid foods were introduced to control- } \\
\text { group infants, based on AAP } \\
\text { recommendations. }\end{array}$ & $\begin{array}{l}\text { Prophylaxis vs Control: NS } \\
\text { Cumulative prevalence: } \\
\text { Prophylaxis vs, Control: NS }\end{array}$ & $\begin{array}{l}\text { - More women in the prophylactic-treated group } \\
\text { withdrew before delivery because of the protocol's } \\
\text { dietary restrictions. As a result, halfway through } \\
\text { recruitment, randomization was changed from } \\
40: 60 \text { (treatment: control) to } 50: 50 \text { with a new } \\
\text { computerized list of random numbers } \\
\text { - Rate of drop-out was significantly different in the } \\
\text { prophylaxis vs. control groups (p<0.0001) } \\
\text { - Significantly more non-White fathers in } \\
\text { prophylaxis group, and trend to more mothers with } \\
\text { low education in control group } \\
\text { - } 6 \text { pairs of twins omitted from control group after } \\
\text { randomization } \\
\text { - BW } 2.9 \% \text { lower in term, singletons in prophylactic } \\
\text { group (P=0.044); Overall, BW similar between } \\
\text { groups } \\
\text { - Smoking during postpartum was significantly } \\
\text { different between the prophylactic and control } \\
\text { groups } \\
\text { - Some missing data, but power calculation } \\
\text { suggests the analytic } \mathrm{N} \text { is sufficient to test } \\
\text { hypotheses. } \\
\text { - Physician making diagnosis was aware of } \\
\text { exposure status; outcomes required both lab tests } \\
\text { and observations at multiple time points, with } \\
\text { similar results for "probable" and "definite" } \\
\text { diagnoses. } \\
\text { - No pre-registered data analysis plan. } \\
\text { Summary: } \\
\text { There was no relationship between maternal diet } \\
\text { and allergic rhinitis in the child, since both } \\
\text { prophylaxis and control groups evidenced similar } \\
\text { (cumulative and period) prevalence } \\
\text { of allergic rhinitis. }\end{array}$ \\
\hline Zeiger, 1995³; U.S & Food(s) or Food Group(s): & Significant: & Key Confounders: \\
\hline
\end{tabular}




Study and Participant
Characteristics
RCT
Baseline N=379 Analytic
$N=165$

\section{See Zeiger, $1989^{35}$}

\section{Intervention/Exposure and}

Outcomes

- Control ( $n=106)$ : Mothers encouraged to follow standard diets during 3 rd trimester of pregnancy and lactation

- Prophylaxis $(n=59)$ : Avoided all milk (dairy), egg, and peanut products, concentrated soy foods (i.e., tofu), $\leq 2$ servings/d wheat during 3rd trimester of pregnancy and lactation

- Prenatal vitamins plus supplemented with a total of $1500 \mathrm{mg} / \mathrm{d} \mathrm{Ca}$

Both groups encouraged to feed human milk for $\geq 4-6$ mo

\section{Outcome:}

Atopic dermatitis: Eczema

at $7 \mathrm{y}$ of age

Prophylactic infants used casein hydrolysate (Nutramigen) for supplementation or weaning, and avoided solid foods for 6 mo; cow milk, corn, soy, citrus, and wheat, for $12 \mathrm{mo}$; and egg, peanut, and fish, for $24 \mathrm{mo}$. Infants in the control groups Enfamil, a cow milk-based whey infant formula.

Solid foods were introduced to controlgroup infants, based on AAP

recommendations.

\section{Results}

\section{Non-significant:}

Atopic dermatitis

- Period prevalence, $\mathrm{P}=\mathrm{NS}$

- Cumulative prevalence, $P=N S$
Confounding, Study Limitations, and Summary of Findings

Age, Race/Ethnicity, SES, Smoking, Family history, HMF, CFB, Pets

\section{OFCs accounted for:}

Sex

\section{Limitations:}

- Unclear if the participants that were lost to followup (since the baseline study) were any different than participants that completed the study. More participants were lost to follow up in the prophylaxis group than the control group

- More women in the prophylactic-treated group withdrew before delivery because of the protocol's dietary restrictions. As a result, halfway through recruitment, randomization was changed from 40:60 (treatment: control) to 50:50 with a new computerized list of random numbers

- Rate of drop-out was significantly different in the prophylaxis vs. control groups $(p<0.0001)$.

- Significantly more non-White fathers in prophylaxis group, and trend to more mothers with low education in control group

- 6 pairs of twins omitted from control group after randomization

- BW $2.9 \%$ lower in term, singletons in prophylactic group ( $\mathrm{P}=0.044)$; Overall, BW similar between groups

- Smoking during postpartum was significantly different between the prophylactic and control groups

\section{Summary:}

Maternal avoidance of milk (dairy), egg, and peanut products, avoid concentrated soy foods, and limited 
Study and Participant

Characteristics
Intervention/Exposure and

Outcomes
Results
Confounding, Study Limitations, and Summary of Findings

intake of wheat during the 3rd trimester of

pregnancy through lactation and reduced infant food allergen exposure during CFB did not affect the prevalence of food allergy in the child at $7 \mathrm{y}$, replicating previously reported findings at $3 y$ and

\section{Non-Randomized Controlled Trials}

\section{Herrmann, $1996^{10}$; Germany \\ NRCT}

Baseline $\mathrm{N}=150$ Analytic $\mathrm{N}=138$

- Age: 30.5

- Race/Ethnicity: NR

- SES:

- Maternal Education:

- Low: 26.8\%

- Medium: $~ 17.3 \%$

- High: 56.0\%

- Characteristics reported with no differences by exposure
o Family history
o BW
$\circ \mathrm{HMF}$
$\circ \mathrm{CFB}$

Women with a positive family history for atopic diseases (asthma, atopic eczema, allergic rhinitis) participated. Atopic heredity was

\section{Food(s) or Food Group(s):}

- Group A: Diet free of any cow milk of pregnancy and during the time of exclusive human milk feeding, $n=30$

- Group B: Diet free of any cow milk and egg protein from delivery through lactation, $n=33$

- HF: Mothers from groups A-C who stopped or supplemented human milk feeding with hydrolysate formula, $\mathrm{n}=34$ (Originally, 8 were in group $\mathrm{A}$, 12 in group $B$ and 14 in group $C$ )

All women recommended to adhere to a prudent diet rich in cereals, fresh fruits and vegetables.

\section{Dietary assessment methods:}

A history of food consumption (as an extended 24-h recall) and of general nutritional habits preceded the recommended nutritional changes. Information about possible product substitutes for milk and eggs along with recipes were given and motivationally discussed by the nutritionist. Mothers and egg protein in the third trimeste

- Group C: Diet not restricted, $\mathrm{n}=41$

\section{Significant:}

\section{Non-significant:}

Atopic dermatitis

- Cumulative prevalence (0-12mo postpartum), $\mathrm{P}=\mathrm{NS}$

- Group A: Cases $n=6$

- Group B: Cases $n=6$

- Group C: Cases n=5

- Prevalence 0-6mo postpartum, $\mathrm{P}=\mathrm{NS}$

- Group A: Cases n=2

- Group B: Cases n=2

- Group C: Cases $n=1$

- Cumulative prevalence in HF group relative to intervention groups, $P=0.095$

HF: Cases $n=10$

\section{$4 y$.}

\section{Key confounders accounted for:}

Family history

\section{OFCs accounted for:}

None

\section{Limitations:}

- Adherence rates by group was NR, but the authors mention that only $67 \%$ of the participants were on a diet the entire time and it took 14 days for the "nutritional change" to become a habit.

- History of atopic dermatitis was statistically different between certain groups (Group A vs. C, $\mathrm{p}<0.003$ ).

- Outcome assessors aware of the assigned interventions.

\section{Summary:}

Maternal avoidance of cow milk and egg protein during pregnancy and exclusive human milk feeding during lactation was not associated with prevalence of atopic dermatitis in the child during the first year of life (6 or $12 \mathrm{mo}$ ). 


\section{Study and Participant}

Characteristics

evaluated and infants from

families with one of the first

relative (i.e. parent or sibling)

with atopy were included.

\section{Intervention/Exposure and}

Outcomes

decided themselves which nutritiona

intervention they were ready to tolerate.

Outcome: Atopic Dermatitis

at $1,3,6$, and 12 mo

Outcome assessment methods:

Infants were seen regularly by three

pediatricians trained in atopy diagnosis

at $1,3,6$ and $12 \mathrm{mo}$. The parents

underwent a structured interview

regarding their infant's nutrition,

diseases and relevant allergy signs and

symptoms during the past period, with

special emphasis placed on skin

conditions in the infant's examinations.

Atopic dermatitis defined as: a history

of dry skin and at least three of the

following typical morphological

characteristics: pruritic dermatitis at

typical sites, facial or extensor

eczematous or lichenified dermatitis,

periauricular fissures.

\section{Lactation \\ Randomized Controlled Trials}

Jirapinyo, 2013 ${ }^{37}$; Thailand Food(s) or Food Group(s):

RCT

Baseline $\mathrm{N}=62$ Analytic $\mathrm{N}=62$

- Control $(n=32)$ : liberal diets

- No Dairy ( $n=30)$ : dairy-restricted die

with $1 \mathrm{~g} / \mathrm{d}$ Ca supplements

from delivery until 4 mo postpartum

- Age: 28.7y

Dietary assessment methods:

\section{Results}

Confounding, Study Limitations, and Summary of Findings

\footnotetext{
- Race/Ethnicity: NR
}

\section{Significant:}

Atopic dermatitis

- at $4 \mathrm{mo}, \mathrm{P}<0.05$

- Control: Cases $n=8(25 \%)$

- No Dairy: Cases n=2 (6.7\%)

Non-significant:

Atopic dermatitis

\section{Key confounders accounted for:}

Age, Race/Ethnicity, Family history, BW, Delivery mode, HMF, CFB timing

\section{OFCs accounted for:}

Sex 


\section{Study and Participant}

\section{Characteristics}

\section{- SES: NR}

- Other characteristics reported with no differences by exposure

- Family history

o BW

Delivery mode

- HMF ( $100 \%$ exclusive)

- CFB timing (none)

All mothers were allergic
Intervention/Exposure and

\section{Outcomes}

Women were randomly assigned to groups. In dairy-restricted group, authors educated mothers to restrict cows' milk and dairy products and foods containing cow milk protein.

\section{Outcome: Atopic Dermatitis}

at $7 \mathrm{~d}, 1 \mathrm{mo}$, and $4 \mathrm{mo}$

Outcome assessment methods:

Examination for atopic dermatitis in infants was blindly performed by $\geq 2$ investigators at $7 \mathrm{~d}, 1 \mathrm{mo}$, and $4 \mathrm{mo}$
Confounding, Study Limitations, and Summary of Findings

Results $\quad \begin{aligned} & \text { of Findings } \\ & \text { of }\end{aligned}$

- at $7 d, P=N S$

Limitations:

- Participants were not blinded. While the outcome assessors were blinded, it is unclear if the investigators were blinded.

- It is unclear if adherence to the diet was assessed in intervention and control groups.

- No published protocol.

\section{Summary:}

Consuming a dairy-restricted diet during the first 4 mo of lactation reduced the incidence of atopic dermatitis among infants at $4 \mathrm{mo}$. 
Table 4. Description of evidence on the relationship between maternal diet during pregnancy and lactation and risk of child allergic rhinitis $^{\mathrm{xiv}, \mathrm{xv}}$

\begin{tabular}{|c|c|c|c|}
\hline $\begin{array}{l}\text { Study and Participant } \\
\text { Characteristics }\end{array}$ & $\begin{array}{l}\text { Intervention/Exposure and } \\
\text { Outcomes }\end{array}$ & Results & $\begin{array}{l}\text { Confounding, Study Limitations, and } \\
\text { Summary of Findings }\end{array}$ \\
\hline \multicolumn{4}{|l|}{ Pregnancy } \\
\hline \multicolumn{4}{|l|}{ Randomized Controlled Trials } \\
\hline $\begin{array}{l}\text { - Age: NR } \\
\text { - Race/Ethnicity: NR } \\
\text { - SES: NR } \\
\text { - Significant differences by } \\
\text { exposure: HMF, CFB } \\
\text { - No differences by exposure: } \\
\text { Smoking (infant exposure), } \\
\text { Family history, BW, Animal/ } \\
\text { Pets/Farming exposure }\end{array}$ & $\begin{array}{l}\text { Food(s) or Food Groups(s): } \\
\text { - Non-Diet: Mothers encouraged to } \\
\text { follow standard diets, } n=108 \\
\text { randomized, } 102 \text { adhered and } \\
\text { completed follow-up } \\
\text { - Diet: Strictly milk- and egg-free diet, } \\
\mathrm{n}=104,79 \text { adhered and completed } \\
\text { follow-up } \\
\text { O Supplemented with casein } \\
\text { hydrolysate formula and Ca } \\
\text { supplement for a total of } 1200 \mathrm{mg} / \mathrm{d} \\
\text { Ca } \\
\text { from } 28 w k \text { through delivery } \\
\text { Both groups encouraged to feed human } \\
\text { milk } \\
\text { By their own choice, many mothers } \\
\text { limited milk and egg intake during } \\
\text { lactation: at both 6wk and } 3 \text { mo, } \mathrm{D} \text {-true } \\
\text { group had greater proportion of mothers } \\
\text { with complete avoidance }(\mathrm{P}<0.05) \text { or }\end{array}$ & $\begin{array}{l}\text { Significant: } \\
\text { Non-Significant: } \\
\text { Probable rhinoconjunctivitis, } \mathrm{P}=\mathrm{NS}\end{array}$ & $\begin{array}{l}\text { Key confounders accounted for: } \\
\text { Family history, Animal/Pets/ Farming } \\
\text { exposure } \\
\text { OFCs accounted for: } \\
\text { None } \\
\text { Limitations: } \\
\text { - Age at weaning: D-true (7.06mo), ND- } \\
\text { true (5.98mo), P<0.005; Full or partial } \\
\text { HMF at } 6 \text { wk and } 6 \text { mo, P=NS } \\
\text { - Infant CM introduction by } 6 \text { mo: D-true } \\
\text { (55.1\%), ND-true ( } 81.2 \%), P<0.0005 \\
\text { - Unclear whether sex was balanced: } \\
\text { D-true (32F, } 47 \mathrm{M}), \text { ND-true (40F, } \\
62 \mathrm{M}) \\
\text { - One pair of twins included, not } \\
\text { accounted for in analyses (group NR) } \\
\text { - } 3 \text { infants born preterm, all in D group } \\
\text { - } ~ 50 \% \text { of women adhered to casein } \\
\text { hydrolysate }\end{array}$ \\
\hline
\end{tabular}

xiv \pm indicates values of Mean \pm SD unless otherwise noted

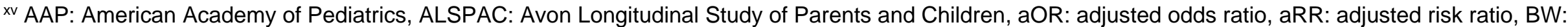

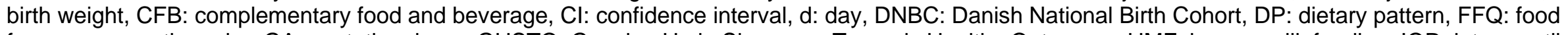

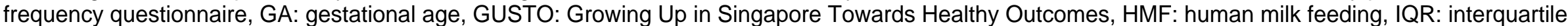

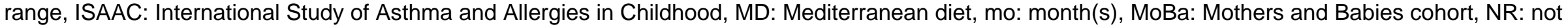

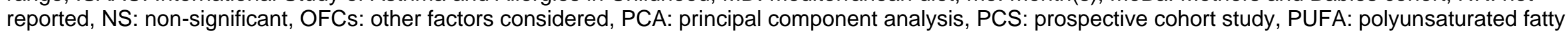
acid, RCT: randomized control trial, Ref: Reference, SES: socioeconomic status, SPT: skin prick test, T\#: tertile, wk: week(s), y: year(s) 


\begin{tabular}{|c|c|c|}
\hline $\begin{array}{l}\text { Study and Participant } \\
\text { Characteristics }\end{array}$ & $\begin{array}{l}\text { Intervention/Exposure and } \quad \text { Results } \\
\text { Outcomes }\end{array}$ & $\begin{array}{l}\text { Confounding, Study Limitations, and } \\
\text { Summary of Findings }\end{array}$ \\
\hline $\begin{array}{l}\text { at least one of the family } \\
\text { member (husband or child) }\end{array}$ & $\begin{array}{l}\text { Tow intake of } \leq 2 \mathrm{dL} / \mathrm{wk} \text { milk and } \leq 2 \\
\text { eggs/wk (P<0.0001). } \\
\text { Diet during lactation: Mothers that } \\
\text { asked for information about diet during } \\
\text { lactation were informed about a "low } \\
\text { allergenic diet" regimen, allowing up to } \\
2 \text { dl of milk per day and two eggs per } \\
\text { week. In the diet group, } 10 \text { mothers also } \\
\text { continued to keep a strictly milk- and } \\
\text { egg-free diet after delivery. The } \\
\text { difference in consumption of these } \\
\text { foods between the groups was } \\
\text { statistically significant. } \\
\text { Outcome: } \\
\text { Probable rhinoconjunctivitis at } 18 m o \\
\text { outcome assessment methods: } \\
\text { All babies who demonstrated any signs } \\
\text { and symptoms suggesting atopic } \\
\text { disease or allergy before the age of } 18 \\
\text { mo were examined by a senior pediatric } \\
\text { allergist. The selection of examination } \\
\text { was based on the information from the } \\
\text { questionnaires, available case records, } \\
\text { babies demonstrating positive SPTs on } \\
\text { any occasion were examined, as well } \\
\text { as babies with a history of either otitis } \\
\text { media, bronchitis, or pseudocroup on } \\
\text { two or more occasions, and babies } \\
\text { whose parents or the nurse had } \\
\text { of physical examination at } 18 \text { mo }\end{array}$ & $\begin{array}{l}\text { - Differences in HMF duration and CFB } \\
\text { could have influenced the outcome } \\
\text { - Attrition of } 15 \% \text { still left }>180 \\
\text { participants required by estimate from } \\
\text { power calculation } \\
\text { - Lost to follow-up was higher in the D } \\
\text { group than the ND group } \\
\text { - No pre-registered data analysis plan } \\
\text { Summary: } \\
\text { Maternal avoidance of milk and eggs } \\
\text { during } 28 w k \text { gestation through delivery } \\
\text { was not associated with probable } \\
\text { rhinoconjunctivitis in the child at } 18 m o \text {. }\end{array}$ \\
\hline
\end{tabular}




\begin{tabular}{|c|c|c|c|}
\hline $\begin{array}{l}\text { Study and Participant } \\
\text { Characteristics }\end{array}$ & $\begin{array}{l}\text { Intervention/Exposure and } \\
\text { Outcomes }\end{array}$ & Results & $\begin{array}{l}\text { Confounding, Study Limitations, and } \\
\text { Summary of Findings }\end{array}$ \\
\hline & $\begin{array}{l}\text { was to score whether the child up to the } \\
\text { age of that age had demonstrated } \\
\text { definite, probable, possible, or no signs } \\
\text { of atopic diseases. A question mark } \\
\text { after atopic dermatitis points out that } \\
\text { some but not all of the diagnostic } \\
\text { features were present. }\end{array}$ & & \\
\hline $\begin{array}{l}\text { Lilja, } 1989^{14} \text {; Sweden } \\
\text { Cluster RCT } \\
\text { Baseline N=171 Analytic N=162 } \\
\text { - Age: 28.7y } \\
\text { - Race/Ethnicity: NR } \\
\text { - SES: NR } \\
\text { - Characteristics reported with } \\
\text { no difference by exposure } \\
\text { ○ Smoking } \\
\text { o Family history } \\
\text { ○ HMF } \\
\text { O CFB timing, types } \\
\text { o Animal/Pets/Farming } \\
\text { exposure } \\
\text { Women in this study had a } \\
\text { history of respiratory allergy } \\
\text { symptoms after exposure to } \\
\text { animal danders (cat and dog) } \\
\text { and/or tree or grass pollens }\end{array}$ & $\begin{array}{l}\text { Food(s) or Food Group(s): } \\
\text { - High: Consumed "normal" amounts of } \\
\text { hens' egg and cows' milk, } n=83 \\
\text { - Reduced: Strictly reduced ingestion of } \\
\text { egg and dairy products (milk, yogurt, } \\
\text { butter, cheese, etc.), } n=79 \\
\text { o Reduced A-Group: Women chose } \\
\text { to continue reduced diet through } \\
2 \text { mo postpartum ( } n=25 \text { ) } \\
\text { - Reduced B-Group: Women } \\
\text { adhered to study design and } \\
\text { stopped reduced diet after delivery } \\
\text { ( } n=54 \text { ) } \\
\text { from } 28 w k \text { gestation until delivery (until } \\
2 \text { mo postpartum for A-Group) } \\
\text { Dietary assessment methods: } \\
\text { Women were randomly assigned to } \\
\text { groups. In reduced group, no foods } \\
\text { obviously containing egg or cows' milk } \\
\text { were allowed, but small amounts of } \\
\text { these foods, such as bread brushed } \\
\text { with egg or ordinary margarines, were } \\
\text { allowed. }\end{array}$ & $\begin{array}{l}\text { Non-significant: } \\
\text { Rhinoconjunctivitis by } 18 \mathrm{mo} \\
\text { - There were no significant } \\
\text { differences between any groups } \\
\text { (High, Reduced, A-Group, B- } \\
\text { Group) in cumulative prevalence of } \\
\text { obvious, probable, or possible } \\
\text { rhinoconjunctivitis. }\end{array}$ & $\begin{array}{l}\text { Key confounders accounted for: } \\
\text { Age, Race/Ethnicity, SES, Smoking, } \\
\text { Family history, HMF, CFB, Pets } \\
\text { OFCs accounted for: } \\
\text { Child sex } \\
\text { Limitations: } \\
\text { - There was a significantly higher } \\
\text { incidence of atopic eczema and } \\
\text { higher IgE levels before week } 28 \text { in } \\
\text { the 'reduced' group. } \\
\text { - Data <18mo measured but NR. } \\
\text { - } 4 \text { pairs of twins included, but family } \\
\text { relation is not accounted for in } \\
\text { analyses. } \\
\text { - Although a cluster RCT, it seems like } \\
\text { the data analysis was conducted at } \\
\text { the individual level. } \\
\text { Summary: } \\
\text { Higher (vs reduced) intake of egg and } \\
\text { dairy products during pregnancy did not } \\
\text { impact child's rhinoconjunctivitis risk at } \\
18 \text { mo. }\end{array}$ \\
\hline
\end{tabular}




\begin{tabular}{|c|c|c|c|}
\hline $\begin{array}{l}\text { Study and Participant } \\
\text { Characteristics }\end{array}$ & $\begin{array}{l}\text { Intervention/Exposure and } \\
\text { Outcomes }\end{array}$ & Results & $\begin{array}{l}\text { Confounding, Study Limitations, and } \\
\text { Summary of Findings }\end{array}$ \\
\hline & $\begin{array}{l}\text { Rhinoconjunctivitis } \\
\text { at } 2,6,12 \text { and } 18 \text { mo } \\
\text { Outcome assessment methods: } \\
\text { All children were examined at } 2,6,12 \text {, } \\
\text { and } 18 \text { mo. The physicians who } \\
\text { performed the physical examination at } \\
18 \text { mo were unaware of the mothers' } \\
\text { diet during the trial and of previously } \\
\text { performed SPT and in-vitro analyses in } \\
\text { the mother and child. The cumulative } \\
\text { incidence of signs of atopic diseases } \\
\geq 18 \text { mo was evaluated by a } \\
\text { questionnaire and the physical } \\
\text { examination into the following groups: } \\
\text { obvious atopic, probable atopic, } \\
\text { possible atopic, and the type of atopic } \\
\text { symptom was noted. }\end{array}$ & & \\
\hline \multicolumn{4}{|l|}{ Prospective Cohort Studies } \\
\hline 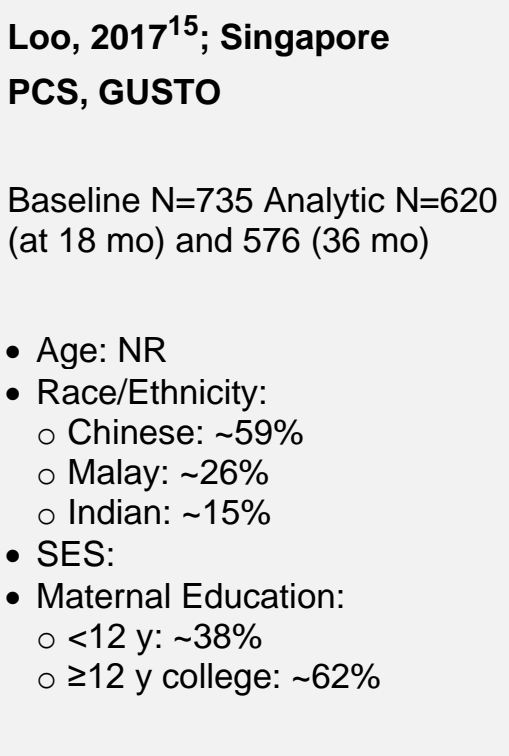 & $\begin{array}{l}\text { Food(s) or Food Group(s): } \\
\text { Dietary pattern adherence } \\
\text { - VFR: Higher score indicated higher } \\
\text { intakes of vegetables, fruit, and rice } \\
\text { - SFN: Higher score indicated higher } \\
\text { intakes of seafood and noodles } \\
\text { - PCP: Higher score indicated higher } \\
\text { intakes of pasta, cheese, and } \\
\text { processed meat } \\
\text { at } 26-28 \text { wk gestation } \\
\text { Dietary assessment methods: } \\
\text { Dietary intake was assessed at } 26-28 \\
\text { weeks of pregnancy which was } \\
\text { conducted by trained clinical staff with } \\
\text { the use of the 5-stage multiple pass }\end{array}$ & $\begin{array}{l}\text { Significant: } \\
\text { Non-significant: } \\
\text { All dietary patterns at } 18 \text { mo and } 3 y \text {, } \\
P=N S\end{array}$ & $\begin{array}{l}\text { Key confounders accounted for: } \\
\text { SES, Family history, Race/ethnicity } \\
\text { OFCs accounted for: } \\
\text { Sex } \\
\text { Limitations: } \\
\text { - At least one key confounder not } \\
\text { adjusted for } \\
\text { - No information on potential co- } \\
\text { exposures } \\
\text { - Proportions of or reasons for } \\
\text { missingness NR } \\
\text { - Physician-diagnosis for eczema; } \\
\text { women probably unaware of own } \\
\text { dietary pattern. }\end{array}$ \\
\hline
\end{tabular}




\begin{tabular}{|c|c|c|c|}
\hline $\begin{array}{l}\text { Study and Participant } \\
\text { Characteristics }\end{array}$ & $\begin{array}{l}\text { Intervention/Exposure and } \\
\text { Outcomes }\end{array}$ & Results & $\begin{array}{l}\text { Confounding, Study Limitations, and } \\
\text { Summary of Findings }\end{array}$ \\
\hline $\begin{array}{l}\text { Reported but not tested by } \\
\text { exposure: Family history, SES }\end{array}$ & $\begin{array}{l}\text { interviewing technique. Dietary patterns } \\
\text { were derived by principal component } \\
\text { extraction with Varimax rotation on the } \\
68 \text { food groups. Three factors (i.e. } \\
\text { dietary patterns) were retained } \\
\text { Outcome: } \\
\text { Rhinitis at } 18 \text { mo and } 3 \text { y } \\
\text { Outcome assessment methods: } \\
\text { "Rhinitis" was based on a positive } \\
\text { response to the question "Has your } \\
\text { child ever had sneezing, running nose, } \\
\text { blocked or congested nose, snoring or } \\
\text { noisy breathing during sleep or when } \\
\text { awake that has lasted for } 2 \text { or more } \\
\text { weeks duration?" Study team members } \\
\text { called the participants who reported } \\
\text { rhinitis to collect information on the } \\
\text { number of episodes of rhinitis and the } \\
\text { duration of each episode. A case prior } \\
\text { to } 18 \text { mo required a single episode that } \\
\text { lasted for at least } 4 \text { weeks or two or } \\
\text { more episodes each lasting at least } 2 \\
\text { weeks. New cases of rhinitis after } 18 \\
\text { mo were defined by one or more } \\
\text { episodes lasting at least } 2 \text { weeks. }\end{array}$ & & $\begin{array}{l}\text { - Outcomes collected at } 3,6,9,12,15 \text {, } \\
18,24 \text {, and } 36 \text { mo, but only } 18,36 \text { mo } \\
\text { reported because SPT data collected } \\
\text { then } \\
\text { - Although the outcome was based on } \\
\text { diagnosed eczema, it was still self- } \\
\text { reported } \\
\text { - Mothers who participated in the study } \\
\text { differed on the following } \\
\text { characteristics from those that did not: } \\
\text { race/ethnicity and maternal education } \\
\text { levels } \\
\text { - Pre-registered data analysis plan NR } \\
\text { Summary: } \\
\text { Maternal consumption of any dietary } \\
\text { pattern during } 26-28 \text { wk pregnancy was } \\
\text { not associated with risk of rhinitis in the } \\
\text { child at } 18 \text { mo or } 3 \mathrm{y} \text {. }\end{array}$ \\
\hline $\begin{array}{l}\text { Shaheen, 200929; U.K. } \\
\text { PCS, ALSPAC } \\
\text { Baseline } \mathrm{N}=12008 \text { Analytic } \\
\mathrm{N}=9516 \\
\text { - Aae: }\end{array}$ & $\begin{array}{l}\text { Food(s) or Food Group(s): } \\
\text { Dietary pattern (Factor Analysis): } \\
\text { - Health conscious pattern: High } \\
\text { consumption of salad, fruit, fruit juices, } \\
\text { rice, pasta, oat/bran based breakfast } \\
\text { cereals, fish, pulses, cheese, and non- } \\
\text { white bread. Low consumption of } \\
\text { white bread. }\end{array}$ & $\begin{array}{l}\text { Significant: } \\
\text { Non-significant: } \\
\text { Hay Fever (Allergic rhinitis) at } 7.5 y \\
\text { - Health conscious DP, } P=N S \\
\text { - Traditional } D P, P=N S \\
\text { - Processed DP, } P=N S \\
\text { - Confectionary, } P=N S\end{array}$ & $\begin{array}{l}\text { Key confounders accounted for: } \\
\text { Age, Race/ethnicity, SES, Smoking, } \\
\text { Family history, GA, BW, HMF, } \\
\text { Animals/pets/farming exposure } \\
\text { OFCs accounted for: }\end{array}$ \\
\hline
\end{tabular}




\begin{tabular}{|c|c|c|c|}
\hline $\begin{array}{l}\text { Study and Participant } \\
\text { Characteristics }\end{array}$ & $\begin{array}{l}\text { Intervention/Exposure and } \\
\text { Outcomes }\end{array}$ & Results & $\begin{array}{l}\text { Confounding, Study Limitations, and } \\
\text { Summary of Findings }\end{array}$ \\
\hline 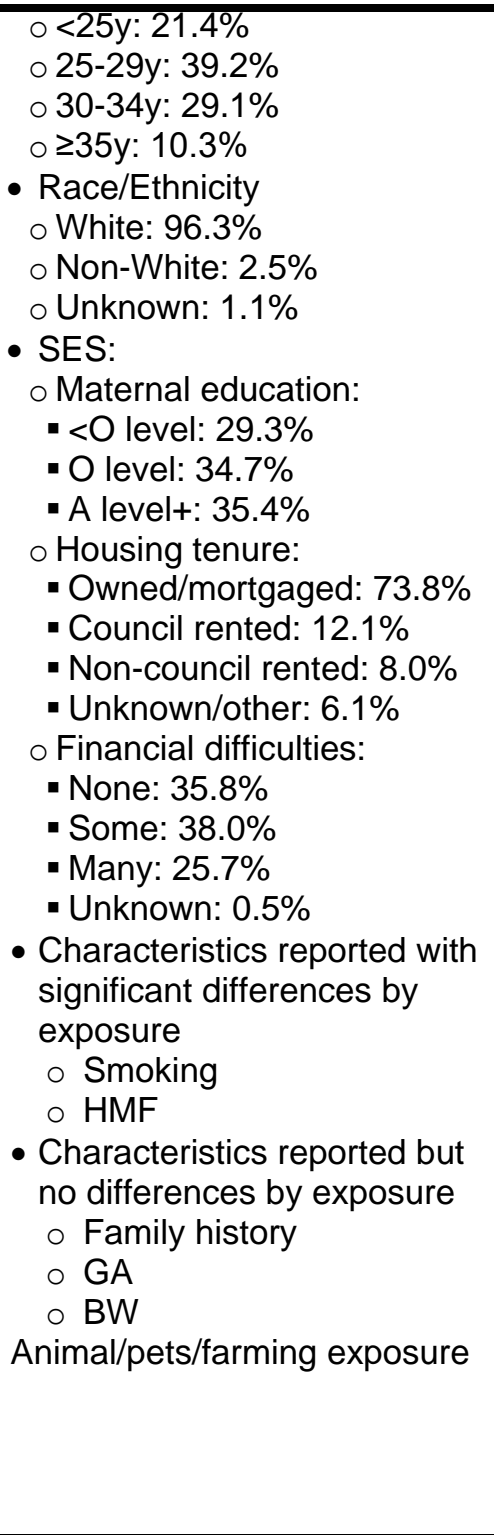 & $\begin{array}{l}\text { Traditional pattern: High consumption } \\
\text { of potatoes (not chips), vegetables, } \\
\text { red meat, and poultry. } \\
\text { - Processed pattern: High consumption } \\
\text { of meat pies, sausages, burgers, fried } \\
\text { foods, pizza, chips, roast potatoes, } \\
\text { white bread, eggs, and baked beans. } \\
\text { Low consumption of non-white bread. } \\
\text { - Confectionery pattern: High } \\
\text { consumption of chocolate, sweets, } \\
\text { biscuits, cakes/buns, puddings, and } \\
\text { crisps. } \\
\text { - Vegetarian pattern: High consumption } \\
\text { of meat substitutes, pulses, nuts, and } \\
\text { herbal tea. Low consumption of } \\
\text { poultry and red meat. } \\
\text { at } 32 \text { wk gestation } \\
\text { Dietary assessment methods: } \\
43 \text {-item FFQ at } 32 \text { wk gestation, based } \\
\text { on FFQ validated in a British population. } \\
5 \text { dietary patterns were identified in the } \\
\text { cohort using PCA: "health conscious", } \\
\text { "traditional", "processed", "vegetarian" } \\
\text { and "confectionery". DP scores were } \\
\text { expressed in standard deviation units. } \\
\text { Each mother was represented in each } \\
\text { of these } 5 \text { mutually independent scores. } \\
\text { Outcome: } \\
\text { Hay fever (Allergic Rhinitis) } \\
\text { Outcome assessment methods: } \\
\text { When the children were } 7.5 \text { y old the } \\
\text { mothers were asked: "Has your child }\end{array}$ & - Vegetarian, $\mathrm{P}=\mathrm{NS}$ & $\begin{array}{l}\text { Child sex, Indoor and outdoor } \\
\text { environment } \\
\text { Limitations: } \\
\text { - At least one key confounder was not } \\
\text { controlled for } \\
\text { - Authors adjusted for post-exposure } \\
\text { variables } \\
\text { - FFQ was not formally validated, but } \\
\text { was based on a validated FFQ } \\
\text { - Proportions of missing participants } \\
\text { differ substantially across exposures } \\
\text { and the analysis is unlikely to have } \\
\text { removed the risk of bias arising from } \\
\text { the missing data } \\
\text { - Majority of outcomes were subjective } \\
\text { and the outcomes were assessed by } \\
\text { participants, who were aware of the } \\
\text { exposure received } \\
\text { - No pre-registered data analysis plan } \\
\text { Summary: } \\
\text { Greater adherence to dietary patterns } \\
\text { during pregnancy, identified using PCA, } \\
\text { was not associated with allergic rhinitis } \\
\text { at } 2.5 \text { and } 7.5 \text { y of age. }\end{array}$ \\
\hline
\end{tabular}




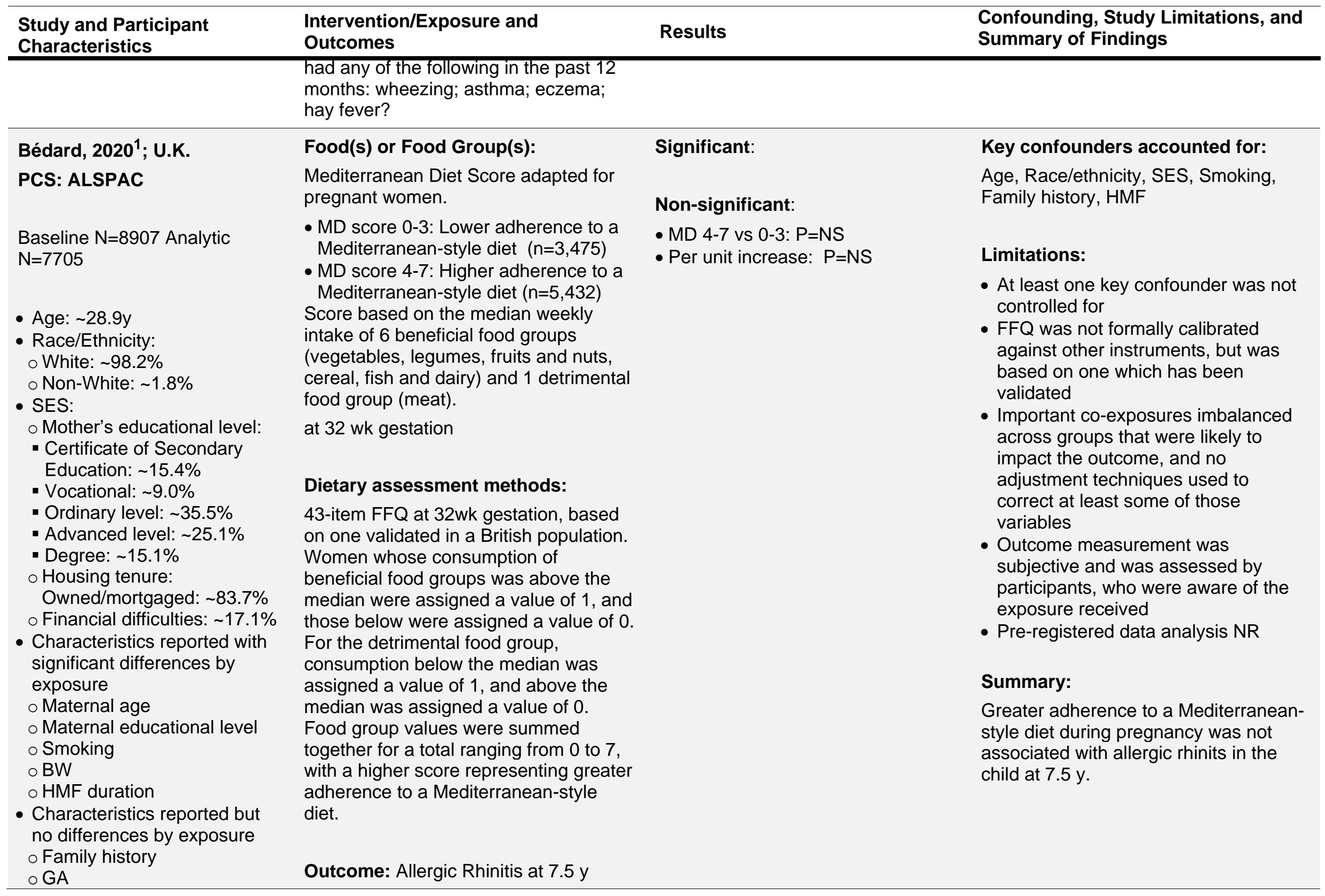




\begin{tabular}{|c|c|c|c|}
\hline $\begin{array}{l}\text { Study and Participant } \\
\text { Characteristics }\end{array}$ & $\begin{array}{l}\text { Intervention/Exposure and } \\
\text { Outcomes }\end{array}$ & Results & $\begin{array}{l}\text { Confounding, Study Limitations, and } \\
\text { Summary of Findings }\end{array}$ \\
\hline o Race/ethnicity & $\begin{array}{l}\text { Outcome assessment methods: } \\
\text { Hay fever in children at } 7.5 \text { y defined by } \\
\text { a positive answer to the question: "Has } \\
\text { your child had any of the following in the } \\
\text { past } 12 \text { months: wheezing with } \\
\text { whistling; eczema; hay fever?" }\end{array}$ & & \\
\hline $\begin{array}{l}\text { Bertelsen, 2014; Norway } \\
\text { PCS, MoBa } \\
\text { Baseline N=40614 Analytic } \\
\text { N=40614 } \\
\text { - Age: } \\
\text { o <20-24y: } 9 \% \\
\text { o } \geq 35 y: 18 \% \\
\text { - Race/Ethnicity: NR } \\
\text { - SES: } \\
\text { o Single: } 3 \% \\
\text { o Maternal Education: } \\
\text { - <High school: } 6 \% \\
\text { - >4y college: } 23 \% \\
\text { Reported but not tested by } \\
\text { exposure: Smoking, Family } \\
\text { history, BW, GA, Delivery } \\
\text { mode, HMF }\end{array}$ & $\begin{array}{l}\text { Food(s) or Food Group(s): } \\
\text { - Unexposed: No consumption of } \\
\text { probiotic milk and yogurt, } n=25,572 \\
(63 \%) \\
\text { - Exposed: Maternal consumption of } \\
\text { probiotic milk and yogurt n=15,042 } \\
\text { (37\%) } \\
\text { during pregnancy (at } 22 \text { wk) } \\
\text { Dietary assessment methods: } \\
\text { Intake of milk-based probiotic products } \\
\text { during pregnancy was recorded in the } \\
\text { FFQ. The women were asked how often } \\
\text { they consumed milk and yogurt, clearly } \\
\text { distinguishing probiotic milk and yogurt } \\
\text { from other milk items. Reported } \\
\text { pregnancy } \\
\text { consumption across all probiotic milk } \\
\text { products was categorized into one } \\
\text { dichotomous variable for any intake } \\
\text { versus no intake and one } 3-\text { level } \\
\text { variable based on intake in milliliters per } \\
\text { day categorized as "none," "13.0-28.3 } \\
\text { mL/d," and " }>28.4 \text { mL/d" } \\
\text { Outcome: }\end{array}$ & 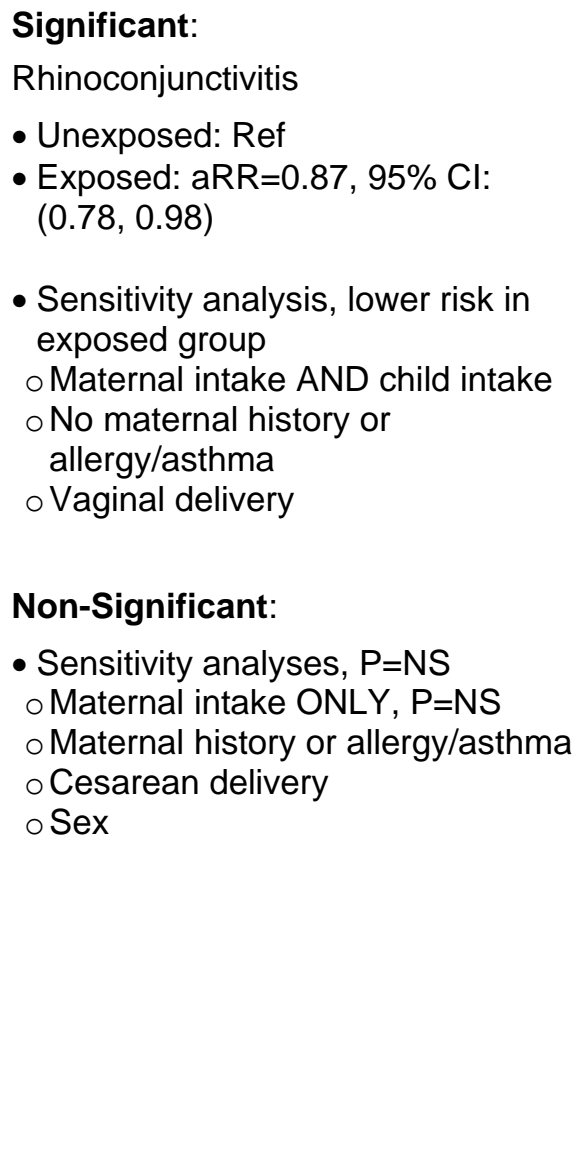 & $\begin{array}{l}\text { Key confounders accounted for: } \\
\text { Age, SES, Smoking, Family history, } \\
\text { HMF, Sex, Delivery mode } \\
\text { OFCs accounted for: } \\
\text { Child sex } \\
\text { Limitations: } \\
\text { - At least one key confounder not } \\
\text { adjusted for } \\
\text { - Based on the exposure data } \\
\text { availability, only a sub-set of women } \\
\text { from MoBa were included in this } \\
\text { study. Mothers who participated in } \\
\text { this study were different than the rest, } \\
\text { on the following characteristics: } \\
\text { education, smoking and parity } \\
\text { - Outcome measurement was } \\
\text { subjective and were assessed by } \\
\text { participants, who were aware of the } \\
\text { exposure received } \\
\text { Summary: } \\
\text { Maternal consumption of probiotic milk } \\
\text { and yogurt during pregnancy was } \\
\text { associated with lower risk of }\end{array}$ \\
\hline
\end{tabular}




\begin{tabular}{|c|c|c|c|}
\hline $\begin{array}{l}\text { Study and Participant } \\
\text { Characteristics }\end{array}$ & $\begin{array}{l}\text { Intervention/Exposure and } \\
\text { Outcomes }\end{array}$ & Results & $\begin{array}{l}\text { Confounding, Study Limitations, and } \\
\text { Summary of Findings }\end{array}$ \\
\hline & Rhinoconjunctivitis at $18-36 \mathrm{mo}$ & & $\begin{array}{l}\text { rhinoconjunctivitis in the child at } 18-36 \\
\text { mo. }\end{array}$ \\
\hline & $\begin{array}{l}\text { Outcome assessment methods: } \\
\text { A child was classified as having } \\
\text { rhinoconjunctivitis based on a } \\
\text { mother's "yes" response to a question } \\
\text { about "allergy affecting eyes or nose, } \\
\text { e.g., hay fever" on the 36-month } \\
\text { questionnaire }\end{array}$ & & $\begin{array}{l}\text { Results from sensitivity analyses were } \\
\text { mixed. }\end{array}$ \\
\hline $\begin{array}{l}\text { Bunyavanich, 20144; U.S. } \\
\text { PCS, Project Viva } \\
\text { Baseline N=2128 Analytic } \\
\mathrm{N}=1277 \\
\text { - Age: } 32.3 \text { (from other Project } \\
\text { Viva data) } \\
\text { - Race/Ethnicity: White: } 69 \% \\
\text { - SES: } \\
\text { o Maternal Education: } \\
\text { o } \geq \text { college graduate: } 69.3 \% \\
\text { o Household income } \geq \$ 70 \mathrm{~K} \text { : } \\
63.0 \% \\
\text { - Reported but not tested by } \\
\text { exposure: Family history }\end{array}$ & $\begin{array}{l}\text { Food(s) or Food Group(s): } \\
\text { Maternal consumption of major food } \\
\text { allergens (servings/d z scores) } \\
\text { - Peanut: } \\
\circ 1^{\text {st }} \text { trimester: } 0.34 \pm 0.44 \\
\circ 2^{\text {nd }} \text { trimester: } 0.36 \pm 0.43 \\
\text { - Milk: } \\
\circ 1^{\text {st }} \text { trimester: } 1.16 \pm 1.04 \\
\circ 2^{\text {nd }} \text { trimester: } 1.50 \pm 1.82 \\
\text { - Wheat: } \\
\circ 1^{\text {st }} \text { trimester: } 2.65 \pm 1.48 \\
\circ 2^{\text {nd }} \text { trimester: } 2.69 \pm 1.44 \\
\text { - Egg: } \\
\circ 1^{\text {st }} \text { trimester: } 0.32 \pm 0.30 \\
\circ 2^{\text {nd }} \text { trimester: } 0.33_{ \pm} 0.30 \\
\text { - Soy: } \\
\circ 1^{\text {st }} \text { trimester: } 0.08 \pm 0.27 \\
\circ 2^{\text {nd }} \text { trimester: } 0.08 \pm 0.28 \\
\text { at } 10 \text { wk and } 26-28 w k \text { gestation } \\
\text { Dietary assessment methods: } \\
\text { Maternal dietary assessments at the } \\
\text { first and second trimester visits were } \\
\text { based on a validated } 166 \text {-item semi- } \\
\text { quantitative FFQ modified for }\end{array}$ & $\begin{array}{l}\text { Significant: } \\
\text { Maternal 1st trimester milk intake } \\
\text { (per z score): } \\
\text { aOR=0.85, } 95 \% \mathrm{Cl}:(0.74,0.97) \\
\text { Stratification: No parental atopy } \\
\text { Maternal } 1 \text { st trimester milk intake } \\
\text { (per z score): } \\
\text { aOR=0.76, } 95 \% \mathrm{Cl} \text { : }(0.59,0.99) \\
\text { Non-significant: } \\
\text { Maternal } 1 \text { st trimester intake of } \\
\text { peanut, wheat, egg, or soy, } \mathrm{P}=\mathrm{NS} \\
\text { Maternal } 2 \text { nd trimester intake of any } \\
\text { food allergens, } \mathrm{P}=\mathrm{NS}\end{array}$ & $\begin{array}{l}\text { Key confounders accounted for: } \\
\text { (Race/Ethnicity adjusted in a secondary } \\
\text { model), SES, Family history, HMF } \\
\text { OFCs accounted for: } \\
\text { Child sex } \\
\text { Limitations: } \\
\text { - At least one key confounder not } \\
\text { adjusted for } \\
\text { - Critical co-exposures NR } \\
\text { - Mothers who participated in the study } \\
\text { were different than those who were } \\
\text { lost to follow-up on the following } \\
\text { characteristics: maternal race, college } \\
\text { education, income, parental atopy } \\
\text { - Proportions of and reasons for } \\
\text { missingness NR by exposure } \\
\text { - Multiple exposure outcome } \\
\text { comparisons were assessed without } \\
\text { using an appropriate p-value } \\
\text { correction } \\
\text { - Self-reported exposure and outcome } \\
\text { (for clinical symptoms) }\end{array}$ \\
\hline
\end{tabular}




\begin{tabular}{|c|c|c|c|}
\hline $\begin{array}{l}\text { Study and Participant } \\
\text { Characteristics }\end{array}$ & $\begin{array}{l}\text { Intervention/Exposure and } \\
\text { Outcomes }\end{array}$ & Results & $\begin{array}{l}\text { Confounding, Study Limitations, and } \\
\text { Summary of Findings }\end{array}$ \\
\hline & $\begin{array}{l}\text { pregnancy. The total servings per day } \\
\text { of each major food allergen (peanut, } \\
\text { milk, wheat, egg, and soy) were } \\
\text { calculated by summing the servings per } \\
\text { day of the foods on the FFQ containing } \\
\text { these respective food allergens. } \\
\text { Outcome: } \\
\text { Allergic rhinitis at } 7.9 y \\
\text { Outcome assessment methods: } \\
\text { Current allergic rhinitis was defined as } \\
\text { positive if a mother reported that her } \\
\text { child had a runny nose or sneezing } \\
\text { apart from colds in the past } 12 \text { mo. Ever } \\
\text { allergic rhinitis, was defined as positive } \\
\text { if a mother reported a doctor's } \\
\text { diagnosis of each respective condition } \\
\text { in the child in any questionnaire since } \\
\text { birth. }\end{array}$ & $\begin{array}{l}\text { trimester milk with no parental atopy) } \\
\text { and allergic rhinitis. }\end{array}$ & $\begin{array}{l}\text { - Pre-registered data analysis plan NR } \\
\text { Summary: } \\
\text { Higher maternal consumption of milk } \\
\text { during the } 1 \text { st trimester of pregnancy } \\
\text { was associated with reduced risk of } \\
\text { allergic rhinitis in the child at } 7.9 \mathrm{y} \text {. } \\
\text { Maternal intake of peanut, wheat, egg, } \\
\text { or soy in the } 1 \text { st trimester or of any } \\
\text { major food allergens in the } 2 \text { nd } \\
\text { trimester were not associated with risk } \\
\text { of allergic rhinitis in the child at } 7.9 \mathrm{y} \text {. }\end{array}$ \\
\hline $\begin{array}{l}\text { Willers, } 2007^{\mathbf{3 1}} \text {; U.K. } \\
\text { PCS } \\
\text { Baseline } \mathrm{N}=1751 \text { Analytic } \\
\mathrm{N}=1212 \\
\text { Of } 1751 \text { women who completed } \\
\text { FFQ during pregnancy, } 1212 \\
\text { completed follow-up when } \\
\text { children were } 5 \mathrm{y} \text {. } \\
\\
\text { - Age at recruitment: } 29.9 \mathrm{y}, \\
\text { 95\% Cl: (29.6, 30.2) } \\
\text { - Race/Ethnicity: NR }\end{array}$ & $\begin{array}{l}\text { Food(s) or Food Group(s): } \\
\text { Apple consumption by tertile } \\
\text { - T1: } 0-1 / \text { wk, } n=398 \\
\text { - T2: } 1-4 / w k, n=427 \\
\text { - T3: }>4 / \text { wk, } n=384 \\
\text { at } \sim 32 \text { wk gestation } \\
\text { Fish consumption } \\
\text { - Never, } n=107 \\
\bullet<1 / \text { wk, } n=255 \\
\bullet \geq 1 / \text { wk, } n=831 \\
\text { at } \sim 32 \text { wk gestation }\end{array}$ & $\begin{array}{l}\text { Significant: } \\
\text { Doctor confirmed hay fever } \\
\text { - Oily fish intake, Cases } \mathrm{n}=68, \mathrm{P} \text { for } \\
\text { trend=0.043 } \\
0 \text { Never: Ref } \\
0<1 / \mathrm{wk} \text { : aOR=0.66, } 95 \% \mathrm{Cl}:(0.34 \text {, } \\
1.28) \\
0 \geq 1 / \mathrm{wk} \text { : aOR=0.28, } 95 \% \mathrm{Cl}:(0.06 \text {, } \\
1.19) \\
\text { Non-significant: } \\
\text { Current hay fever medication } \\
\text { - Oily fish intake, Cases } \mathrm{n}=44, \mathrm{P} \text { for } \\
\text { trend=NS }\end{array}$ & $\begin{array}{l}\text { Key confounders accounted for: } \\
\text { Age, SES, Smoking (Mother, } \\
\text { Household), Family history, BW, HMF } \\
\text { OFCs accounted for: } \\
\text { Child sex } \\
\text { Limitations: } \\
\text { - At least one key confounder not } \\
\text { adjusted for } \\
\text { - Mothers who participated in the study } \\
\text { were of higher SES and had slightly } \\
\text { higher consumption of fruits, green }\end{array}$ \\
\hline
\end{tabular}




\begin{tabular}{|c|c|c|c|}
\hline $\begin{array}{l}\text { Study and Participant } \\
\text { Characteristics }\end{array}$ & $\begin{array}{l}\text { Intervention/Exposure and } \\
\text { Outcomes }\end{array}$ & Results & $\begin{array}{l}\text { Confounding, Study Limitations, and } \\
\text { Summary of Findings }\end{array}$ \\
\hline $\begin{array}{l}\text { - SES: } \\
\text { o Partner from non-manual } \\
\text { social class: } 62.7 \% \\
\text { o Age left full time education: } \\
\text { Median=18.5y, IQR=(16.0, } \\
21.0) \\
\text { - Characteristics reported but } \\
\text { no test for differences by } \\
\text { exposure } \\
\text { o Smoking } \\
\text { o Family history } \\
\text { o BW } \\
\text { o HMF (Ever) }\end{array}$ & $\begin{array}{l}\text { Oliy fish consumption } \\
\text { - Never, n=629 } \\
\text { - }<1 / \text { wk, } n=414 \\
\text { - } \geq 1 / \text { wk, n=161 } \\
\text { at } ~ 32 \text { wk gestation } \\
\text { Dietary assessment methods: } \\
\text { Semi-quantitative, } 150 \text {-item FFQ, } \\
\text { divided into } 20 \text { food groups to capture } \\
\text { intake over the previous } 2-3 \text { mo. } \\
\text { Responses were categorized as rarely } \\
\text { or never, } 1-2 \text { times/mo, and separate } \\
\text { categories for } 1-7 \text { d/wk. Food amounts } \\
\text { were recorded as } 1 \text { to } \geq 5 \text { measures per } \\
\text { day. The number of measures/d was } \\
\text { multiplied by the number of d/wk to } \\
\text { obtain the total measures/wk. } \\
\text { The food groups of interest in this study } \\
\text { were total fruit, citrus/kiwi fruit, apples, } \\
\text { total vegetables, green leafy } \\
\text { vegetables, pure fruit juice, whole grain } \\
\text { products, total fish, total oily fish, total } \\
\text { fat from dairy products and exclusive } \\
\text { butter versus margarine/low fat spread } \\
\text { used as spread. The total number of } \\
\text { measures per week was divided into } \\
\text { tertiles for total fruit, citrus/kiwi fruit, } \\
\text { apples, total vegetables, green leafy } \\
\text { vegetables, pure fruit juice, whole grain } \\
\text { products and into the categories never, } \\
\text { less than once a week, and once or } \\
\text { more a week for total fish and total oily } \\
\text { fish. To facilitate extrapolation to the } \\
\text { general population, subdivisions of food } \\
\text { intakes into tertiles were derived from } \\
\text { all of the women completing the FFQ }\end{array}$ & $\begin{array}{l}\text { Ever had hay fever } \\
\text { - Oily fish intake, Cases } n=111, P \text { for } \\
\text { trend=NS }\end{array}$ & $\begin{array}{l}\text { leafy vegetables, whole grain } \\
\text { products and fish. They also had } \\
\text { fewer respiratory symptoms. } \\
\text { - Multiple exposure outcome } \\
\text { comparisons were assessed without } \\
\text { using an appropriate p-value } \\
\text { correction. } \\
\text { Summary: } \\
\text { Higher maternal oily fish intake was } \\
\text { associated with lower odds of doctor- } \\
\text { confirmed hay fever in the child at age } \\
5 y, \text { but was not associated with current } \\
\text { hay fever medication or ever having } \\
\text { had hay fever. }\end{array}$ \\
\hline
\end{tabular}




\begin{tabular}{|c|c|c|c|}
\hline $\begin{array}{l}\text { Study and Participant } \\
\text { Characteristics }\end{array}$ & $\begin{array}{l}\text { Intervention/Exposure and } \\
\text { Outcomes }\end{array}$ & Results & $\begin{array}{l}\text { Confounding, Study Limitations, and } \\
\text { Summary of Findings }\end{array}$ \\
\hline & $\begin{array}{l}\text { and not merely those responding at } 5 \\
\text { years. } \\
\text { Outcomes: Asthma, Allergic dermatitis } \\
\text { (eczema), Allergic rhinitis (hay fever) } \\
\text { at } 5 y \\
\text { Outcome assessment methods: } \\
\text { Based on ISAAC core questions on } \\
\text { symptoms of asthma, allergic rhinitis } \\
\text { and atopic eczema, including the } \\
\text { questions: "Has your child ever suffered } \\
\text { from asthma?", "Has this been } \\
\text { confirmed by a doctor?" and "Has your } \\
\text { child received treatment for asthma in } \\
\text { the past } 12 \text { months?" with similar } \\
\text { questions enquired about eczema and } \\
\text { hay fever. }\end{array}$ & & \\
\hline $\begin{array}{l}\text { Maslova, 2012 }{ }^{17} \text {; Denmark } \\
\text { PCS, DNBC } \\
\text { Baseline N=61,908 Analytic } \\
\mathrm{N}=38,570 \\
\text { - Age: } 29.0 \pm 4.0 \mathrm{y} \\
\text { - Race/Ethnicity: NR } \\
\text { - SES: } \\
\text { o SES Position: High-level } \\
\text { proficiencies: 23\% } \\
\text { - Characteristics reported by } \\
\text { exposure but unknown } \\
\text { significance } \\
\text { oSmoking } \\
\text { oFamily history } \\
\text { oGA }\end{array}$ & $\begin{array}{l}\text { Food(s) or Food Group(s): } \\
\text { Peanut and pistachio intake } \\
\text { Tree nut intake } \\
\text { - Group 1: Never } \\
\text { - Group 2: } 1 \mathrm{x} / \mathrm{mo} \\
\text { - Group 3: } 1-3 \mathrm{x} / \mathrm{mo} \\
\text { - Group 4: } \geq 1 \mathrm{x} / \mathrm{wk} \\
\text { once at } 25 \text { wk gestation } \\
\text { Dietary assessment methods: } \\
\text { Peanut and nut intake was assessed } \\
\text { during mid-pregnancy by using a } \\
\text { validated } 360 \text {-item semi-quantitative } \\
\text { FFQ15 that covered intake in the past } \\
\text { 4wk. Specific questions were asked } \\
\text { about snack consumption in the past }\end{array}$ & $\begin{array}{l}\text { Significant: } \\
\text { Peanut and pistachio intake } \\
\text { Ever prescribed allergic rhinitis } \\
\text { ( } \mathrm{n}=38,552), \mathrm{P} \text { for trend=0.001 } \\
\text { - } 1 \text { time/mo vs Never (Ref): } \\
\text { aOR=0.84, } 95 \% \mathrm{Cl}=(0.75,0.95) \\
\text { - } 2-3 \text { times/mo vs Never (Ref): } \\
\text { aOR=0.83, 95\% } \mathrm{Cl}=(0.71,0.96) \\
\bullet \geq 1 \text { time/wk vs Never (Ref): } \mathrm{P}=\mathrm{NS} \\
\text { Non-significant: } \\
\text { Peanut and pistachio intake } \\
\text { Self-reported allergic rhinitis } \\
\text { ( } \mathrm{n}=38,552), \mathrm{P} \text { for trend=0.06 } \\
\bullet \geq 1 \text { time/wk to } 1 \text { time/mo vs Never } \\
\text { (Ref): } \mathrm{P}=\mathrm{NS}\end{array}$ & $\begin{array}{l}\text { Key confounders accounted for: } \\
\text { Age, SES, Smoking, Family history, } \\
\text { HMF } \\
\text { OFCs accounted for: } \\
\text { None } \\
\text { Limitations: } \\
\text { - Total sample size in the text and table } \\
\text { does not match. It is unclear why } \\
\text { additional participants were dropped } \\
\text { from the study } \\
\text { - No differentiation between peanut and } \\
\text { pistachio intake } \\
\text { - Exposure does not include processed } \\
\text { nut products }\end{array}$ \\
\hline
\end{tabular}




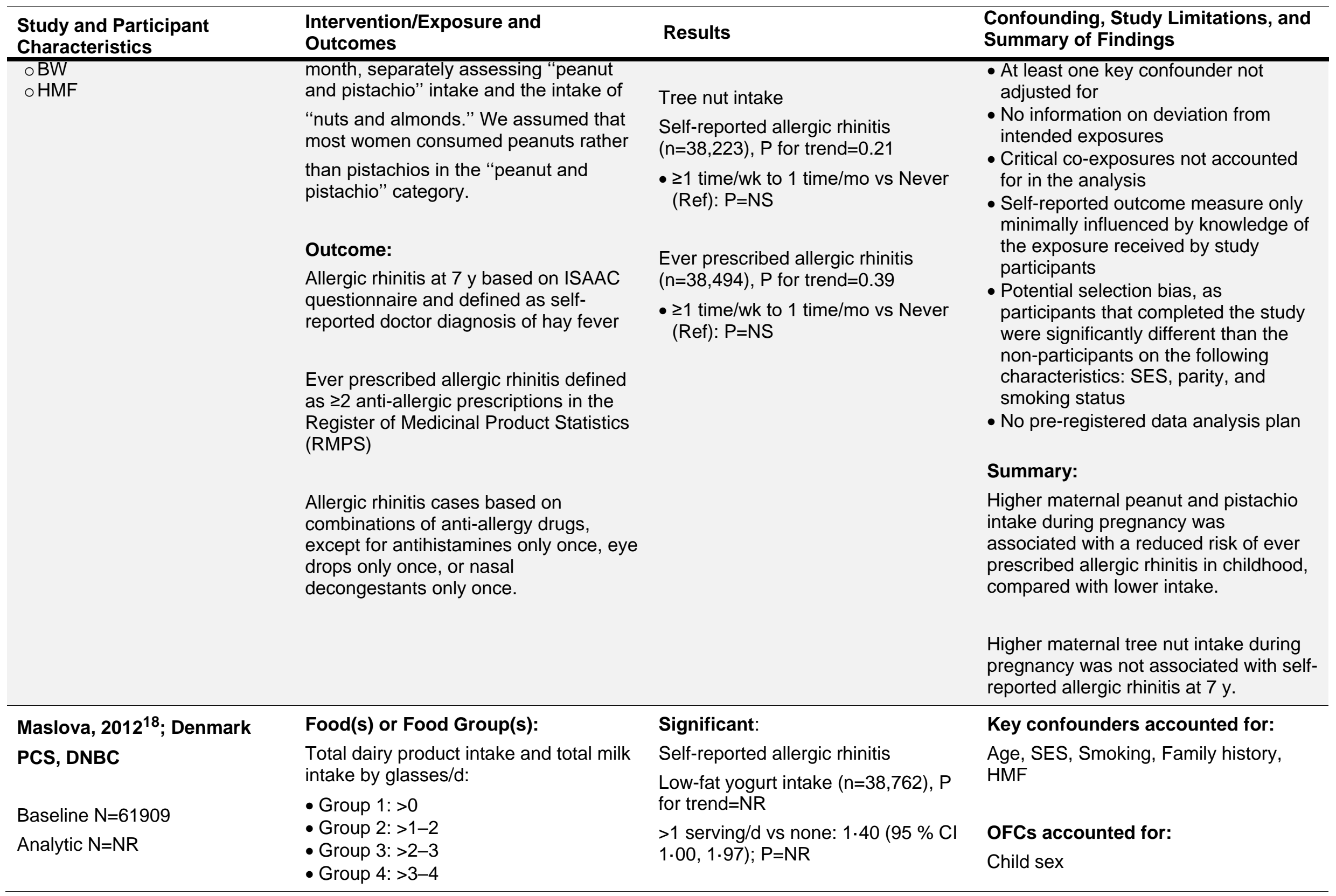




\begin{tabular}{|c|c|c|c|}
\hline $\begin{array}{l}\text { Study and Participant } \\
\text { Characteristics }\end{array}$ & $\begin{array}{l}\text { Intervention/Exposure and } \\
\text { Outcomes }\end{array}$ & Results & $\begin{array}{l}\text { Confounding, Study Limitations, and } \\
\text { Summary of Findings }\end{array}$ \\
\hline 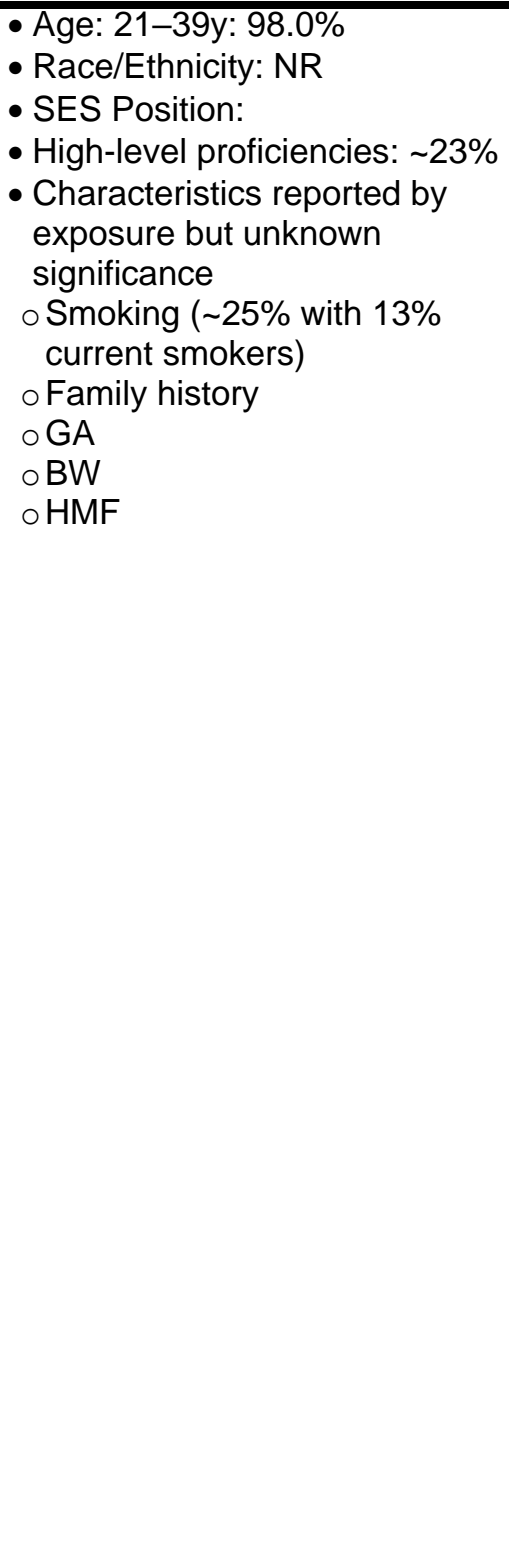 & $\begin{array}{l}\text { - Group 5: }>4-5 \\
\text { - Group 6: }>5 \\
\text { Whole milk intake and semi-skimmed } \\
\text { milk intake by frequency of } \\
\text { consumption: } \\
\text { - Group 1: Never } \\
\text { - Group 2: } 1 \mathrm{x} / \mathrm{mo} \\
\text { - Group 3: } 2.5 \times \text { /mo } \\
\text { - Group 4: } 1.5-3.5 \mathrm{x} / \text { wk } \\
\text { - Group 5: } \geq 5.5 \times / \text { wk } \\
\text { Full-fat yogurt intake and low-fat yogurt } \\
\text { intake by servings/d: } \\
\text { - Group 1: } 0 \\
\text { - Group 2: }>0-0.5 \\
\text { - Group 3: }>0.5-1 \\
\text { - Group 4: }>1 \\
\text { Dietary assessment: } \\
\text { A validated } 360 \text {-item semi-quantitative } \\
\text { FFQ was completed around gestation } \\
\text { week } 25 ; \text { it referred to intake during the } \\
\text { previous } 4 \text { weeks. Dairy product } \\
\text { consumption was recorded in } 8 \\
\text { questions in the FFQ; two of them } \\
\text { asked about consumption of yogurt, in } \\
\text { servings per day (including percentage } \\
\text { of fat, with/without fruit) and } 6 \text { questions } \\
\text { asked about milk consumption (whole } \\
\text { milk, } 1.5 \% \text { milk, } 0 \cdot 5 \% \text { milk, skimmed } \\
\text { milk, churn buttermilk and chocolate } \\
\text { milk) in glasses per day. The FFQ } \\
\text { asked about yogurt with/without fruit to } \\
\text { better estimate carbohydrates. }\end{array}$ & $\begin{array}{l}\text { Further adjustment for other foods } \\
\text { and nutrient intake did not change } \\
\text { the results } \\
\text { Non-Significant: } \\
\text { Associations between total dairy } \\
\text { product, total milk, whole milk, semi- } \\
\text { skimmed milk, and full-fat yogurt and } \\
\text { self-reported allergic rhinitis were } \\
\text { NR. }\end{array}$ & $\begin{array}{l}\text { Limitations: } \\
\text { - At least one key confounder not } \\
\text { controlled for } \\
\text { - Selection into the study was related } \\
\text { (but not very strongly) to exposure } \\
\text { and outcome and this could not be } \\
\text { adjusted for in analyses; } \\
\text { - No information on deviation from } \\
\text { intended exposures; } \\
\text { - Self-reported outcome measure only } \\
\text { minimally influenced by knowledge of } \\
\text { the exposure received by study } \\
\text { participants; } \\
\text { - Potential selection bias, as } \\
\text { participants that completed the study } \\
\text { were significantly different than the } \\
\text { non-participants on the following } \\
\text { characteristics: SES, parity, and } \\
\text { smoking status } \\
\text { - Critical co-exposures not accounted } \\
\text { for in the analysis } \\
\text { - Possible selective reporting of the } \\
\text { findings } \\
\text { - No pre-registered data analysis plan } \\
\text { Summary: } \\
\text { Maternal low-fat yogurt intake during } \\
\text { pregnancy increased the risk of self- } \\
\text { reported allergic-rhinitis at } 7 \text { y. }\end{array}$ \\
\hline
\end{tabular}




\begin{tabular}{|c|c|c|c|}
\hline $\begin{array}{l}\text { Study and Participant } \\
\text { Characteristics }\end{array}$ & $\begin{array}{l}\text { Intervention/Exposure and } \\
\text { Outcomes }\end{array}$ & Results & $\begin{array}{l}\text { Confounding, Study Limitations, and } \\
\text { Summary of Findings }\end{array}$ \\
\hline & $\begin{array}{l}\text { Assuming that the serving sizes were } \\
\text { approximately equal to } 200 \mathrm{ml} \text {, the milk } \\
\text { and yogurt variables were aggregated } \\
\text { to obtain the frequency measures of } \\
\text { total dairy intake. Frequency of milk } \\
\text { intake was quantified by summing all } \\
\text { types of milk and excluding yogurt. For } \\
\text { our analyses, individual types of dairy } \\
\text { product as well as total dairy product, } \\
\text { total milk and total full-fat and low-fat } \\
\text { yoghurt intake was examined. } \\
\text { Once at } 25 \text { wk gestation } \\
\text { Outcome: } \\
\text { Allergic rhinitis at } 7 \text { y based on ISAAC } \\
\text { questionnaire and defined as self- } \\
\text { reported doctor diagnosis of hay fever }\end{array}$ & & \\
\hline $\begin{array}{l}\text { Maslova, 2013 }{ }^{19} \text {; Denmark } \\
\text { PCS, DNBC } \\
\text { Baseline N=28936 Analytic } \\
\mathrm{N}=16867 \\
\text { - Age: } \\
\text { ○ } 20 \mathrm{y}: 0.7 \% \\
\text { ○40y: } 1.2 \% \\
\text { - Race/Ethnicity: NR } \\
\text { - SES: } \\
\text { ○ SES Position: } \\
\text { oHigh-level proficiencies: } \\
22.9 \% \\
\text { o Medium-level proficiencies: } \\
29.4 \% \\
\text { ○ Skilled: } 24.5 \%\end{array}$ & 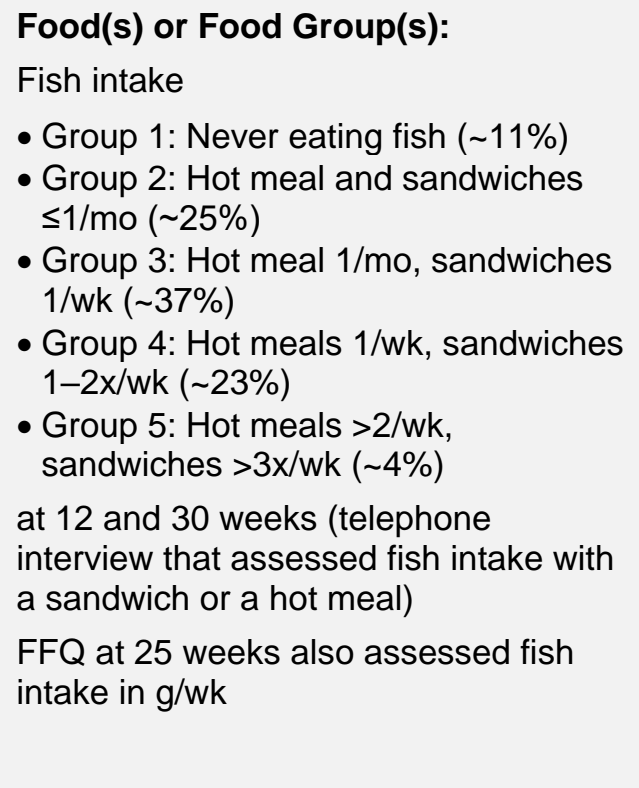 & $\begin{array}{l}\text { Significant: } \\
\text { Self-reported allergic rhinitis } \\
\text { Fish intake ( } n=11,535), \mathrm{P} \text { for } \\
\text { trend=0.01 } \\
\text { - Group } 4 \text { vs Group } 5 \text { (Ref): } \mathrm{P}=\mathrm{NS} \\
\text { - Group } 3 \text { vs Group } 5 \text { (Ref): } \\
\text { aOR=0.73, } 95 \% \mathrm{Cl} \text { ( }(0.54,0.97) \\
\text { - Group } 2 \text { vs Group } 5 \text { (Ref): } \\
\text { aOR=0.68, 95\% Cl: (0.50, 0.94) } \\
\text { - Group } 1 \text { vs Group } 5 \text { (Ref): } P=\mathrm{NS} \\
\text { Non-significant: } \\
\text { Ever prescribed allergic rhinitis } \\
\text { Fish intake ( } n=11,622), P \text { for } \\
\text { trend=0.85 } \\
\text { Groups } 2-5 \text { vs Group } 1 \text { (Ref): } P=N S\end{array}$ & $\begin{array}{l}\text { Key confounders accounted for: } \\
\text { Age, SES, Smoking, Family history, } \\
\text { HMF } \\
\text { OFCs accounted for: } \\
\text { Child sex } \\
\text { Limitations: } \\
\text { - At least one key confounder not } \\
\text { adjusted for } \\
\text { - Selection into the analysis was related } \\
\text { to exposure and may be related to } \\
\text { outcome and this could not be } \\
\text { adjusted for in the analyses } \\
\text { - Participants tended to display } \\
\text { healthier lifestyle habits and a higher } \\
\text { SES compared to non-participants }\end{array}$ \\
\hline
\end{tabular}




\begin{tabular}{|c|c|c|c|}
\hline $\begin{array}{l}\text { Study and Participant } \\
\text { Characteristics }\end{array}$ & $\begin{array}{l}\text { Intervention/Exposure and } \\
\text { Outcomes }\end{array}$ & Results & $\begin{array}{l}\text { Confounding, Study Limitations, and } \\
\text { Summary of Findings }\end{array}$ \\
\hline 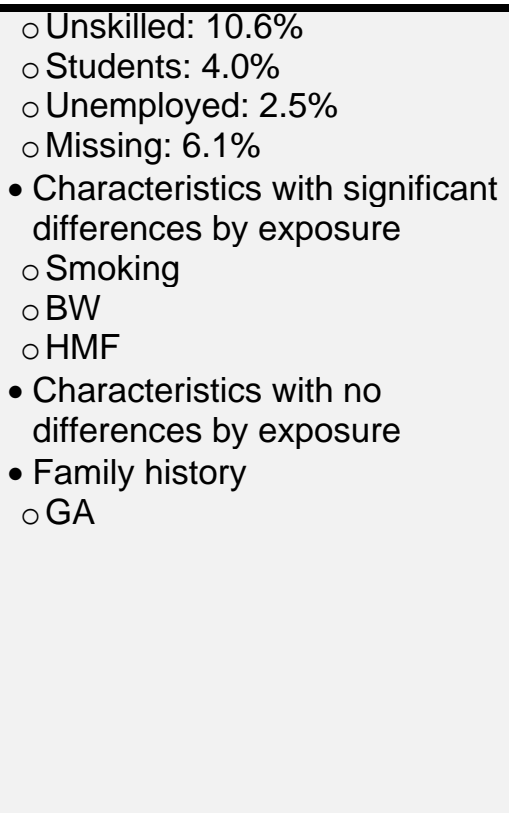 & $\begin{array}{l}\text { Dietary Assessment: } \\
\text { The FFQ asked about intake in the past } \\
4 \text { weeks and has been validated against } \\
7 \mathrm{~d} \text { food diaries and blood and urine } \\
\text { biomarkers for selected nutrient } \\
\text { (protein, retinol, folic acid and n-3 } \\
\text { PUFA) and food (fruit and vegetable) } \\
\text { intake. } \\
\text { Outcome: } \\
\text { Allergic rhinitis at } 7 \text { y based on ISAAC } \\
\text { questionnaire and defined as self- } \\
\text { reported doctor diagnosis of hay fever } \\
\text { Ever prescribed allergic rhinitis defined } \\
\text { as } \geq 2 \text { anti-allergic prescriptions in the } \\
\text { Register of Medicinal Product Statistics } \\
\text { (RMPS) }\end{array}$ & & $\begin{array}{l}\text { - Critical co-exposures not accounted } \\
\text { for in the analysis } \\
\text { - Self-reported outcome measure only } \\
\text { minimally influenced by knowledge of } \\
\text { the exposure received by study } \\
\text { participants } \\
\text { - No pre-registered data analysis plan } \\
\text { Summary: } \\
\text { Maternal fish intake during pregnancy } \\
\text { was not associated with risk of ever } \\
\text { prescribed childhood allergic rhinitis. } \\
\text { Lower maternal fish intake during } \\
\text { pregnancy was associated with a } \\
\text { reduced risk of self-reported childhood } \\
\text { allergic rhinitis compared with higher } \\
\text { fish intake. }\end{array}$ \\
\hline $\begin{array}{l}\text { Maslova, 201320; Denmark } \\
\text { PCS, DNBC } \\
\text { Baseline N=60465 Analytic } \\
\text { N=38398 } \\
\text { - Age: } \\
\text { ○ } 20 y: 1.0 \% \\
\text { o } \geq 40 y: 0.9 \% \\
\text { - Race/Ethnicity: NR } \\
\text { - SES: } \\
\text { o SES Position: } \\
\text { oHigh-level proficiencies: } \\
\text { 20.6\% }\end{array}$ & 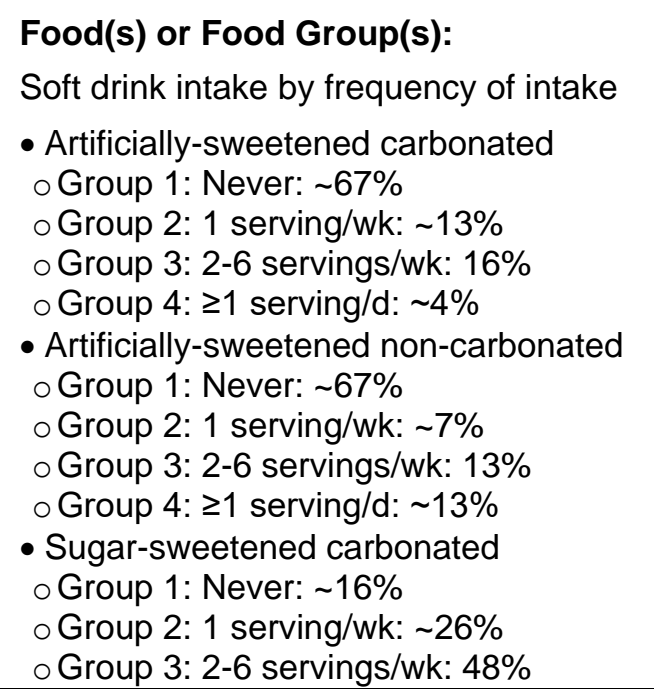 & $\begin{array}{l}\text { Significant: } \\
\text { Self-reported allergic rhinitis } \\
\text { Artificially-sweetened carbonated } \\
\text { soft drink intake ( } n=37,971), P \text { for } \\
\text { trend=0.01 } \\
\text { - Groups } 2-4 \text { vs Group } 1 \text { (Ref): } \\
\text { P=NS } \\
\text { Non-significant: } \\
\text { Self-reported allergic rhinitis } \\
\text { Artificially-sweetened non- } \\
\text { carbonated soft drink intake } \\
\text { (n=37,984), P for trend=0.83 } \\
\text { - Group 1: Ref }\end{array}$ & $\begin{array}{l}\text { Key confounders accounted for: } \\
\text { Age, SES, Smoking, Family history, } \\
\text { Delivery mode, HMF, } \\
\text { Animals/pets/farming } \\
\text { OFCs accounted for: } \\
\text { Child sex } \\
\text { Limitations: } \\
\text { - At least one key confounder not } \\
\text { accounted for } \\
\text { - Participants tended to display } \\
\text { healthier life-style habits compared to } \\
\text { non-participants }\end{array}$ \\
\hline
\end{tabular}




\begin{tabular}{|c|c|c|c|}
\hline $\begin{array}{l}\text { Study and Participant } \\
\text { Characteristics }\end{array}$ & $\begin{array}{l}\text { Intervention/Exposure and } \\
\text { Outcomes }\end{array}$ & Results & $\begin{array}{l}\text { Confounding, Study Limitations, and } \\
\text { Summary of Findings }\end{array}$ \\
\hline $\begin{array}{l}\text { o Medium-level proficiencies: } \\
27.6 \% \\
\text { o Skilled: } 24.1 \% \\
\text { o Unskilled: } 11.3 \% \\
\text { o Students: } 4.0 \% \\
\text { o Unemployed: } 2.4 \% \\
\text { o Missing: } 10.0 \% \\
\text { - Characteristics reported but } \\
\text { no test for differences by } \\
\text { exposure } \\
\text { o Smoking } \\
\text { oFamily history } \\
\text { oGA } \\
\text { oBW } \\
\text { oHMF }\end{array}$ & $\begin{array}{l}\text { ○ Group 4: } \geq 1 \text { serving/d: 10\% } \\
\text { - Sugar-sweetened non-carbonated } \\
\text { o Group 1: Never: 35\% } \\
\text { ○ Group 2: } 1 \text { serving/wk: 15\% } \\
\text { o Group 3: } 2-6 \text { servings/wk: } 28 \% \\
\text { ○ Group 4: } \geq 1 \text { serving/d: } \sim 22 \% \\
\text { assessed once at } 25 \text { wk gestation }\end{array}$ & $\begin{array}{l}\text { - Groups } 2-4, P=N S \\
\text { Sugar-sweetened carbonated soft } \\
\text { drink intake }(n=38,111), P \text { for } \\
\text { trend=0.22 } \\
\text { - Group 1: Ref } \\
\text { - Groups 2-4, } \mathrm{P}=\mathrm{NS} \\
\text { Sugar-sweetened non-carbonated } \\
\text { soft drink intake ( } \mathrm{n}=37,996), \mathrm{P} \text { for } \\
\text { trend=0.72 } \\
\text { - Group 1: Ref } \\
\text { - Groups 2-4, P=NS }\end{array}$ & $\begin{array}{l}\text { - No information on deviation from } \\
\text { intended exposure } \\
\text { - Critical co-exposures not accounted } \\
\text { for in the analysis } \\
\text { - Self-reported outcome measure only } \\
\text { minimally influenced by knowledge of } \\
\text { the exposure received by study } \\
\text { participants } \\
\text { - No pre-registered data analysis plan }\end{array}$ \\
\hline \multicolumn{4}{|l|}{ Pregnancy and Lactation } \\
\hline \multicolumn{4}{|l|}{ Randomized Controlled Trials } \\
\hline $\begin{array}{l}\text { Fukushima, } 1997^{8} \text {; Japan } \\
\text { RCT }\end{array}$ & $\begin{array}{l}\text { Food(s) or Food Group(s): } \\
\text { - CD: Mothers instructed to consume } \\
>200 \mathrm{~mL} / \mathrm{d} \text { cow milk, } n=140 \\
\text { randomized, } n=127 \text { analyzed } \\
\text { - MD: Mothers instructed to consume } \\
>200 \mathrm{~mL} / \mathrm{d} \text { a casein-free, } \\
\text { hypoallergenic formula and to avoid } \\
\text { cow milk and reduce consumption of }\end{array}$ & $\begin{array}{l}\text { Significant: } \\
\text { Note, CD had significantly higher } \\
\text { odds of overall allergies vs MD at } 12 \\
\text { mo, but due to inclusion of asthma } \\
\text { as part of the outcome, these results } \\
\text { do not meet the NESR criteria }\end{array}$ & $\begin{array}{l}\text { Key confounders accounted for: } \\
\text { Smoking, Family history, (CFB for } \\
\text { aOR), Pets }\end{array}$ \\
\hline
\end{tabular}




\begin{tabular}{|c|c|c|c|}
\hline $\begin{array}{l}\text { Study and Participant } \\
\text { Characteristics }\end{array}$ & $\begin{array}{l}\text { Intervention/Exposure and } \\
\text { Outcomes }\end{array}$ & Results & $\begin{array}{l}\text { Confounding, Study Limitations, and } \\
\text { Summary of Findings }\end{array}$ \\
\hline $\begin{array}{l}\text { - Race/Ethnicity: } 100 \% \text { from } \\
\text { Japan } \\
\text { - SES: NR } \\
\text { - Significant differences by } \\
\text { exposure (by design): HMF, } \\
\text { CFB } \\
\text { - No differences by exposure: } \\
\text { Smoking, Family history, BW, } \\
\text { Pets } \\
\text { 76\% had a positive history of } \\
\text { allergy (i.e. both parents or one } \\
\text { parent or sibling only had } \\
\text { history of allergies) }\end{array}$ & $\begin{array}{l}\text { Cow milk products, } \mathrm{n}=140 \text { randomized, } \\
\mathrm{n}=102 \text { analyzed } \\
\text { - AF: Mothers instructed to consume } \\
\text { >200mL/d a casein-free, } \\
\text { hypoallergenic formula and to avoid } \\
\text { cow milk and reduce consumption of } \\
\text { cow milk products, } \mathrm{n}=70 \text { randomized, } \\
\mathrm{n}=54 \text { analyzed (Not randomized, no } \\
\text { relevant comparisons included, except } \\
\text { as co-variates in logistic regressions) } \\
\text { o Also supplemented with } 1000 \text { mg/d } \\
\text { Ca } \\
\text { during late pregnancy through end of } \\
\text { lactation up to 6mo postpartum } \\
\text { From birth to } 6 \text { mo, the infants in the MD } \\
\text { and CD groups were exclusively HMF } \\
\text { or mixed-fed with human milk and } \\
\text { casein-free, hypoallergenic formula } \\
\text { when human milk was insufficient. The } \\
\text { infants in the AF group were mixed-fed } \\
\text { with human milk and a formula with } \\
\text { similar whey:casein ratio as human milk } \\
\text { for the corresponding } 6 \text { mo. Infants who } \\
\text { were fed human milk exclusively from } \\
\text { birth to } 4 \text { mo were excluded from the AF } \\
\text { group. } \\
\text { Dietary assessment methods: } \\
\text { Daily food diary from late pregnancy } \\
\text { until } 6 \text { mo postpartum, recording the } \\
\text { amount of casein-free, hypoallergenic } \\
\text { formula, cow milk, cow milk products, } \\
\text { eggs, meat, and soy products } \\
\text { consumed. }\end{array}$ & $\begin{array}{l}\text { Non-significant: } \\
\text { Allergic rhinitis at any age, } \mathrm{P}=\mathrm{NS} \\
\text { Overall allergies at } 24 \mathrm{mo}, \mathrm{P}=\mathrm{NS} \\
\text { - MD: (Ref) } \\
\text { - CD: aOR=1.75, } 95 \% \mathrm{Cl}:(0.94 \text {, } \\
3.25) \\
\text { Note, odds of overall allergies } \mathrm{NS} \text { at } \\
6 \text { and } 18 \mathrm{mo} \text {. However, due to } \\
\text { inclusion of asthma these results do } \\
\text { not meet the NESR criteria }\end{array}$ & $\begin{array}{l}\text { Limitations: } \\
\text { - Randomization process NR } \\
\text { - MD still consumed cow milk products, } \\
\text { but had lower intake than CD } \\
\text { - Attrition higher in MD group because } \\
\text { women excluded from analysis if they } \\
\text { consumed cow milk } \\
\text { - Proportions of missing data seem to } \\
\text { differ, reasons for missingness NR } \\
\text { - Participants were aware of exposure } \\
\text { status and outcomes were self- } \\
\text { reported } \\
\text { - Eczema logistic regressions only } \\
\text { reported at } 12 \text { and } 24 \text { mo, but data } \\
\text { were collected at all } 4 \text { time points } \\
\text { - Pre-registered data analysis plan NR } \\
\text { Summary: } \\
\text { Consuming diets replacing cow milk } \\
\text { with casein-free hypoallergenic formula } \\
\text { with reduced consumption of cow milk } \\
\text { products from late pregnancy through } \\
\text { lactation did not impact overall } \\
\text { allergies, including atopic eczema, } \\
\text { asthma and allergic rhinitis. }\end{array}$ \\
\hline
\end{tabular}




\begin{tabular}{|c|c|c|c|}
\hline $\begin{array}{l}\text { Study and Participant } \\
\text { Characteristics }\end{array}$ & $\begin{array}{l}\text { Intervention/Exposure and } \\
\text { Outcomes }\end{array}$ & Results & $\begin{array}{l}\text { Confounding, Study Limitations, and } \\
\text { Summary of Findings }\end{array}$ \\
\hline & $\begin{array}{l}\text { Outcomes: } \\
\text { Overall allergies (eczema, asthma, } \\
\text { and/or allergic rhinitis) } \\
\text { at } 24 \text { mo } \\
\text { Outcome assessment methods: } \\
\text { Self-reported by questionnaire. Specific } \\
\text { allergies defined when symptoms } \\
\text { chronically lasted for more than a few } \\
\text { weeks. } \\
\text { Allergic rhinitis was defined as clear } \\
\text { water discharge from the nose. }\end{array}$ & & \\
\hline $\begin{array}{l}\text { Zeiger, } 1989^{35} \text {; U.S. } \\
\text { RCT } \\
\text { Baseline N=379 Analytic N=288 } \\
\text { Power analysis: Yes } \\
\text { Of these families, } 14 \text { in each } \\
\text { group were found to be not } \\
\text { atopic, which eliminated them } \\
\text { from the study } \\
\text { Baseline characteristics for } 288 \\
\text { participants } \\
\text { - Age: } 28.6 y \\
\text { Race/Ethnicity: } \\
\text { o Non-White: } \sim 12.5 \% \\
\text { SES: } \\
\text { O Maternal occupation: white } \\
\text { collar: } 54.0 \% \\
\text { o Family income }<\$ 20,000 / y \text { : } \\
\text { 9.7\% }\end{array}$ & $\begin{array}{l}\text { Food(s) or Food Group(s): } \\
\text { - Control: Mothers encouraged to follow } \\
\text { standard diets during } 3^{\text {rd }} \text { trimester of } \\
\text { pregnancy and lactation, } n=212 \\
\text { O Cow milk-based whey infant } \\
\text { formula provided for } \\
\text { supplementation or weaning } \\
\text { through } 12 \text { mo postpartum. CFB } \\
\text { encouraged: no solids }<4 \text { mo, } \\
\text { cereal at } 4 \text { mo, followed by } \\
\text { vegetables, fruits and egg yolks at } \\
6 \text { mo, meats at } 8 \text { mo, and whole } \\
\text { cow milk and egg whites at } 12 \text { mo. } \\
\text { - Prophylaxis: Instructed to avoid totally } \\
\text { all milk (dairy), egg, and peanut } \\
\text { products, avoid concentrated soy } \\
\text { foods (i.e., tofu), } \leq 2 \text { servings/d wheat, } \\
\text { with other grains to fulfill cereal and } \\
\text { starch requirements during } 3^{\text {rd }} \\
\text { trimester of pregnancy and lactation, } \\
n=167 \\
0 \text { In addition to prenatal vitamins, the } \\
\text { maternal diet was supplemented } \\
\text { with a total of } 1500 \text { mg/d Ca. }\end{array}$ & $\begin{array}{l}\text { Significant: } \\
\text { Non-significant: } \\
\text { Period prevalence of atopic } \\
\text { disorders, } P=N S \text { at each time point } \\
\text { at } 4,12 \text {, and } 24 \mathrm{mo} \\
\text { - Allergic rhinitis } \\
\text { Cumulative prevalence of atopic } \\
\text { disorders, } P=N S \text { at each time point } \\
\text { at } 4,12 \text {, and } 24 \mathrm{mo} \\
\text { - Allergic rhinitis }\end{array}$ & $\begin{array}{l}\text { Limitations: } \\
\text { - Significantly more non-White fathers } \\
\text { in prophylaxis group, and trend to } \\
\text { more mothers with low education in } \\
\text { control group } \\
\text { - } 6 \text { pairs of twins omitted from control } \\
\text { group after randomization } \\
\text { - BW } 2.9 \% \text { lower in term, singletons in } \\
\text { prophylactic group ( } P=0.044 \text { ); Overall, } \\
\text { BW similar between groups } \\
\text { - More women in the prophylactic- } \\
\text { treated group withdrew before } \\
\text { delivery because of the protocol's } \\
\text { dietary restrictions. As a result, } \\
\text { halfway through recruitment, } \\
\text { randomization was changed from } \\
40: 60 \text { (treatment: control) to } 50: 50 \\
\text { with a new computerized list of } \\
\text { random numbers. }\end{array}$ \\
\hline
\end{tabular}




\begin{tabular}{|c|c|c|}
\hline $\begin{array}{l}\text { Study and Participant } \\
\text { Characteristics }\end{array}$ & $\begin{array}{l}\text { Intervention/Exposure and } \\
\text { Outcomes }\end{array}$ & $\begin{array}{l}\text { Confounding, Study Limitations, and } \\
\text { Summary of Findings }\end{array}$ \\
\hline $\begin{array}{l}\text { o Maternal Education: } \\
\text { - } \leq \text { High school: } ~ 13.6 \% \\
\text { - Characteristics reported with } \\
\text { significant differences by } \\
\text { exposure } \\
\text { o BW (term, singletons) } \\
\text { - Characteristics reported with } \\
\text { no differences by exposure } \\
\text { o Smoking } \\
\text { o Family history } \\
\text { o HMF } \\
\text { o CFB } \\
\text { o Animal/Pets/Farming } \\
\text { exposure } \\
\text { Families were included in the } \\
\text { study if at least one parent met } \\
\text { the following criteria: history of } \\
\text { an atopic disorder and specific } \\
\text { lgE by skin or RAST testing. } \\
\text { Serum was obtained from the } \\
\text { participants for total and specific } \\
\text { lgE determinations. Participant } \\
\text { fathers were skin tested to } \\
\text { inhalant antigens at the intake } \\
\text { session. Mothers were skin } \\
\text { tested to foods and inhalants } \\
4 \text { mo postpartum. }\end{array}$ & $\begin{array}{l}\text { A casein hydrolysate infant formula } \\
\text { with low sensitization potential } \\
\text { provided for supplementation or } \\
\text { weaning through } 12 \text { mo } \\
\text { postpartum. CFB encouraged: no } \\
\text { solids <6 mo, non-legume } \\
\text { vegetables, followed by rice cereal } \\
\text { at } 7 \text { mo, meats at } 8 \text { mo, non-citrus } \\
\text { fruits and juices at 9mo, and cow } \\
\text { milk at } 12 \text { mo. Wheat, soy, corn, } \\
\text { and citrus introduced thereafter at } \\
\text { monthly intervals, followed by egg } \\
\text { at } 24 \text { mo and peanuts and fish at } \\
\quad 36 \text { mo. } \\
\text { - Both groups encouraged to feed } \\
\text { human milk for } \geq 4-6 \text { mo } \\
\text { Dietary assessment methods: } \\
\text { Women were randomly assigned to } \\
\text { groups. In addition to instructions } \\
\text { described above, women in prophylaxis } \\
\text { group attended a dietary class was held } \\
\text { before the 3rd trimester by a licensed } \\
\text { dietitian to provide detailed instructions } \\
\text { on the maternal and infant diets, food } \\
\text { lists, recipes, and product sources. } \\
\text { Adherence to the dietary regimen was } \\
\text { ascertained in part by maternal self- } \\
\text { report and daily diaries. } \\
\text { For both groups: } 0.25 \text { mg/d Tri-Vi-Flor } \\
\text { given to infants according to their } \\
\text { pediatrician's preference. Foods } \\
\text { causing documented IgE sensitization } \\
\text { were removed from the infant's diet until } \\
\text { sensitization had waned or were } \\
\text { tolerated on double-blind challenge. } \\
\text { Parents received intensive education on }\end{array}$ & $\begin{array}{l}\text { - Some missing data, but power } \\
\text { calculation suggests the analytic } \mathrm{N} \text { is } \\
\text { sufficient to test hypotheses. } \\
\text { - Physician making diagnosis was } \\
\text { aware of exposure status; outcomes } \\
\text { required both lab tests and } \\
\text { observations at multiple time points, } \\
\text { with similar results for } \\
\text { - "probable" and "definite" diagnoses. } \\
\text { - No pre-registered data analysis plan. } \\
\text { Summary: } \\
\text { There was no relationship between } \\
\text { maternal diet and allergic rhinitis in the } \\
\text { child. }\end{array}$ \\
\hline
\end{tabular}




\begin{tabular}{|c|c|c|c|}
\hline $\begin{array}{l}\text { Study and Participant } \\
\text { Characteristics }\end{array}$ & $\begin{array}{l}\text { Intervention/Exposure and } \\
\text { Outcomes }\end{array}$ & Results & $\begin{array}{l}\text { Confounding, Study Limitations, and } \\
\text { Summary of Findings }\end{array}$ \\
\hline & \multicolumn{2}{|l|}{$\begin{array}{l}\text { reducing environmental allergens and } \\
\text { tobacco smoke from their homes. }\end{array}$} & \\
\hline & \multicolumn{2}{|l|}{$\begin{array}{l}\text { Outcome: Atopic dermatitis, Allergic } \\
\text { rhinitis, Food allergy }\end{array}$} & \\
\hline & \multicolumn{2}{|l|}{ at $1,4,8$, and $12 \mathrm{mo}$} & \\
\hline & \multicolumn{2}{|l|}{ Outcome assessment methods: } & \\
\hline & \multicolumn{2}{|l|}{$\begin{array}{l}\text { Allergic rhinitis was defined as } \\
\text { characteristic sneezing, itching, and/or } \\
\text { rhinorrhea with existing specific IgE and } \\
\text { nasal eosinophilia. Nasal eosinophils } \\
\geq 1+\text { was considered definite, whereas } \\
1 / 2+\text { was suggested as indicating } \\
\text { probable allergic rhinitis. }\end{array}$} & \\
\hline & \multicolumn{2}{|l|}{$\begin{array}{l}\text { Prophylactic infants used casein } \\
\text { hydrolysate (Nutramigen) for } \\
\text { supplementation or weaning, and } \\
\text { avoided solid foods for } 6 \text { mo; cow milk, } \\
\text { corn, soy, citrus, and wheat, for } 12 \text { mo; } \\
\text { and egg, peanut, and fish, for } 24 \text { mo. } \\
\text { Infants in the control groups Enfamil, a } \\
\text { cow milk-based whey infant formula. } \\
\text { Solid foods were introduced to control- } \\
\text { group infants, based on AAP } \\
\text { recommendations. }\end{array}$} & \\
\hline \multirow{2}{*}{$\begin{array}{l}\text { Zeiger, } 1992^{36} \text {; U.S. } \\
\text { RCT }\end{array}$} & \multirow{4}{*}{$\begin{array}{l}\text { Food(s) or Food Group(s): } \\
\text { - Control: Mothers encouraged to follow } \\
\text { standard diets during 3rd trimester of } \\
\text { pregnancy and lactation } \\
\text { - Prophylaxis: Avoided all milk (dairy), } \\
\text { egg, and peanut products, } \\
\text { concentrated soy foods (i.e., tofu), } \leq 2 \\
\text { servings/d wheat during 3rd trimester } \\
\text { of pregnancy and lactation }\end{array}$} & Significant: & Key confounders accounted for: \\
\hline & & Non-Significant: & $\begin{array}{l}\text { Age, Race/Ethnicity, SES, Smoking, } \\
\text { Family history, HMF, CFB, Pets }\end{array}$ \\
\hline \multirow{2}{*}{$\begin{array}{l}\text { Baseline } \mathrm{N}=379 \text { Analytic } \mathrm{N}=242 \\
\text { (at } 3 \text { y) and } 225 \text { (at } 4 \mathrm{y} \text { ) }\end{array}$} & & Period prevalence at 3 and $4 \mathrm{y}$ : & \multirow{2}{*}{$\begin{array}{l}\text { Limitations: } \\
\text { - More women in the prophylactic- } \\
\text { treated group withdrew before } \\
\text { delivery because of the protocol's }\end{array}$} \\
\hline & & Cumulative prevalence: & \\
\hline
\end{tabular}




\begin{tabular}{|c|c|c|c|}
\hline $\begin{array}{l}\text { Study and Participant } \\
\text { Characteristics }\end{array}$ & $\begin{array}{l}\text { Intervention/Exposure and } \\
\text { Outcomes }\end{array}$ & Results & $\begin{array}{l}\text { Confounding, Study Limitations, and } \\
\text { Summary of Findings }\end{array}$ \\
\hline & $\begin{array}{l}\text { o Prenatal vitamins plus supplemented } \\
\text { with a total of } 1500 \mathrm{mg} / \mathrm{d} \mathrm{Ca} \\
\text { Both groups encouraged to feed human } \\
\text { milk for } \geq 4-6 \text { mo } \\
\text { Outcome: } \\
\text { Allergic Rhinitis at } 3 \text { y and } 4 \text { y } \\
\text { Period prevalence: Defined as the } \\
\text { proportion of participants currently } \\
\text { evidencing a measured parameter } \\
\text { Cumulative prevalence: Defined as the } \\
\text { proportion of participants evidencing the } \\
\text { measured parameter at any past or } \\
\text { current time } \\
\text { Prophylactic infants used casein } \\
\text { hydrolysate (Nutramigen) for } \\
\text { supplementation or weaning, and } \\
\text { avoided solid foods for } 6 \text { mo; cow milk, } \\
\text { corn, soy, citrus, and wheat, for } 12 \text { mo; } \\
\text { and egg, peanut, and fish, for } 24 \text { mo. } \\
\text { Infants in the control groups Enfamil, a } \\
\text { cow milk-based whey infant formula. } \\
\text { Solid foods were introduced to control- } \\
\text { group infants, based on AAP } \\
\text { recommendations. }\end{array}$ & Prophylaxis vs, Control: NS & $\begin{array}{l}\text { dietary restrictions. As a result, } \\
\text { halfway through recruitment, } \\
\text { randomization was changed from } \\
40: 60 \text { (treatment: control) to } 50: 50 \\
\text { with a new computerized list of } \\
\text { random numbers } \\
\text { - Rate of drop-out was significantly } \\
\text { different in the prophylaxis vs. control } \\
\text { groups (p<0.0001) } \\
\text { - Significantly more non-White fathers } \\
\text { in prophylaxis group, and trend to } \\
\text { more mothers with low education in } \\
\text { control group } \\
\text { - } 6 \text { pairs of twins omitted from control } \\
\text { group after randomization } \\
\text { - BW } 2.9 \% \text { lower in term, singletons in } \\
\text { prophylactic group (P=0.044); Overall, } \\
\text { BW similar between groups } \\
\text { - Smoking during postpartum was } \\
\text { significantly different between the } \\
\text { prophylactic and control groups } \\
\text { - Some missing data, but power } \\
\text { calculation suggests the analytic } \mathrm{N} \text { is } \\
\text { sufficient to test hypotheses. } \\
\text { - Physician making diagnosis was } \\
\text { aware of exposure status; outcomes } \\
\text { required both lab tests and } \\
\text { observations at multiple time points, } \\
\text { with similar results for "probable" and } \\
\text { "definite" diagnoses. } \\
\text { - No pre-registered data analysis plan. } \\
\text { Summary: } \\
\text { There was no relationship between } \\
\text { maternal diet and allergic rhinitis in the } \\
\text { child, since both prophylaxis and } \\
\text { control groups evidenced similar }\end{array}$ \\
\hline
\end{tabular}




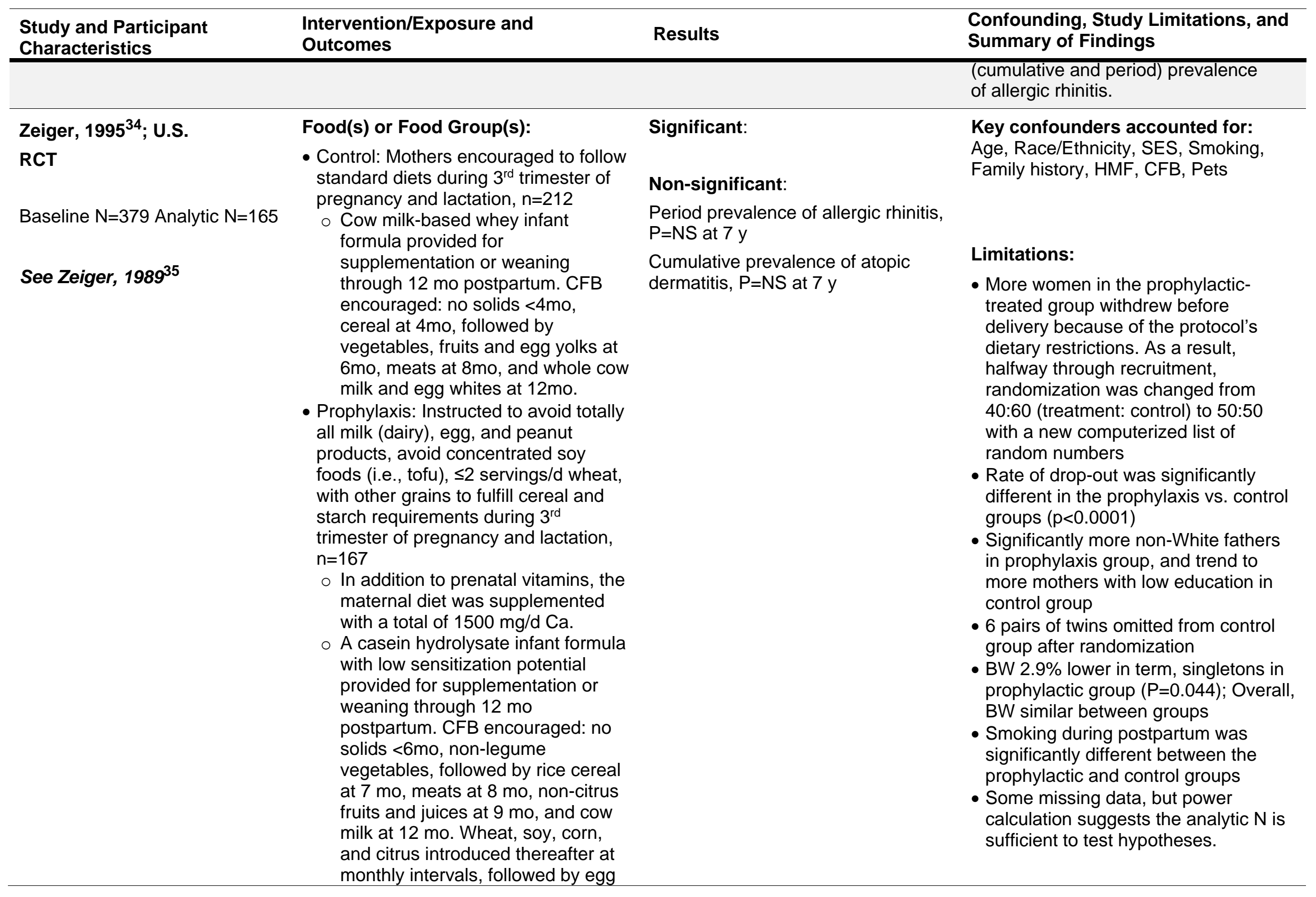




\section{Study and Participant}

Characteristics

\section{Intervention/Exposure and}

Outcomes

at $24 \mathrm{mo}$ and peanuts and fish at

36 mo.

- Both groups encouraged to feed

human milk for $\geq 4-6$ mo

\section{Dietary assessment methods:}

Women were randomly assigned to groups. In addition to instructions described above, women in prophylaxis group attended a dietary class was held before the 3rd trimester by a licensed dietitian to provide detailed instructions on the maternal and infant diets, food

lists, recipes, and product sources. Adherence to the dietary regimen was ascertained in part by maternal selfreport and daily diaries.

For both groups: $0.25 \mathrm{mg} / \mathrm{d}$ Tri-Vi-Flor given to infants according to their pediatrician's preference. Foods causing documented IgE sensitization were removed from the infant's diet until sensitization had waned or were tolerated on double-blind challenge.

Parents received intensive education on reducing environmental allergens and tobacco smoke from their homes.

Outcome: Atopic dermatitis, Allergic rhinitis, Food allergy

at $1,4,8$, and $12 \mathrm{mo}$

\section{Outcome assessment methods:}

Allergic rhinitis was defined as a nasal condition with characteristic symptoms of sneezing, itching, and/or rhinorrhea
Confounding, Study Limitations, and Summary of Findings

- Physician making diagnosis was aware of exposure status; outcomes required both lab tests and observations at multiple time points, with similar results for "probable" and "definite" diagnoses.

- No pre-registered data analysis plan.

\section{Summary:}

Maternal avoidance of milk (dairy), egg, and peanut products, concentrated soy foods, and limited intake of wheat during the $3^{\text {rd }}$ trimester and lactation did not impact the incidence of allergic rhinitis in the child. 
Study and Participant

Characteristics

Intervention/Exposure and

Outcomes

with concurrent specific lge and nasal

eosinophils (NEs)
Confounding, Study Limitations, and Summary of Findings 
Table 5. Description of evidence on the relationship between maternal diet during pregnancy and lactation and risk of child asthmaxvi, xvii

\begin{tabular}{|c|c|c|c|}
\hline $\begin{array}{l}\text { Study and Participant } \\
\text { Characteristics }\end{array}$ & Intervention/Exposure and Outcomes & Results & $\begin{array}{l}\text { Confounding, Study Limitations, and Summary } \\
\text { of Findings }\end{array}$ \\
\hline \multicolumn{4}{|l|}{ Pregnancy } \\
\hline \multicolumn{4}{|l|}{ Prospective Cohort Studies } \\
\hline $\begin{array}{l}\text { Shaheen, } 2009^{29} \text {; U.K. } \\
\text { PCS, ALSPAC } \\
\text { Baseline } \mathrm{N}=12,008 \text { Analytic } \\
\mathrm{N}=9,516 \\
\text { - Age: } \\
\text { ○ <25y: } 21.4 \% \\
\text { ○ } 25-29 \mathrm{y}: 39.2 \% \\
\circ 30-34 \mathrm{y}: 29.1 \% \\
\text { ○ } 235 \mathrm{y}: 10.3 \% \\
\text { - Race/Ethnicity } \\
\text { ○ White: } 96.3 \% \\
\text { ○ Non-White: } 2.5 \% \\
\text { ○ Unknown: } 1.1 \% \\
\text { - SES: } \\
\text { ○ Maternal education: } \\
\text { - <O level: } 29.3 \% \\
\text { - O level: } 34.7 \%\end{array}$ & $\begin{array}{l}\text { Food(s) or Food Group(s): } \\
\text { Dietary pattern: } \\
\text { - Health conscious pattern: High } \\
\text { consumption of salad, fruit, fruit juices, } \\
\text { rice, pasta, oat/bran based breakfast } \\
\text { cereals, fish, pulses, cheese, and non- } \\
\text { white bread. Low consumption of } \\
\text { white bread. } \\
\text { Traditional pattern: High consumption } \\
\text { of potatoes (not chips), vegetables, } \\
\text { red meat, and poultry. } \\
\text { - Processed pattern: High consumption } \\
\text { of meat pies, sausages, burgers, fried } \\
\text { foods, pizza, chips, roast potatoes, } \\
\text { white bread, eggs, and baked beans. } \\
\text { Low consumption of non-white bread. } \\
\text { - Confectionery pattern: High } \\
\text { consumption of chocolate, sweets, } \\
\text { biscuits, cakes/buns, puddings, and } \\
\text { crisps. }\end{array}$ & $\begin{array}{l}\text { Significant: } \\
\text { Non-significant: } \\
\text { Asthma at } 7.5 \mathrm{y} \\
\text { No association with any of the } \\
\text { dietary patterns }\end{array}$ & $\begin{array}{l}\text { Key confounders accounted for: } \\
\text { Age, Race/ethnicity, SES, Smoking, Family history, } \\
\text { GA, BW, HMF, Animals/pets/farming exposure } \\
\text { OFCs accounted for: } \\
\text { Child sex, Indoor and outdoor environment } \\
\text { Limitations: } \\
\text { - At least one key confounder was not controlled for } \\
\text { - Authors adjusted for post-exposure variables } \\
\text { - Start of follow up and exposure do not coincide } \\
\text { and a potentially important amount of follow-up } \\
\text { time is missing from analyses } \\
\text { - FFQ was not formally validated, but was based on } \\
\text { a validated FFQ } \\
\text { - Proportions of missing participants differ } \\
\text { substantially across exposures and the analysis is }\end{array}$ \\
\hline
\end{tabular}

xvi \pm indicates values of Mean \pm SD unless otherwise noted

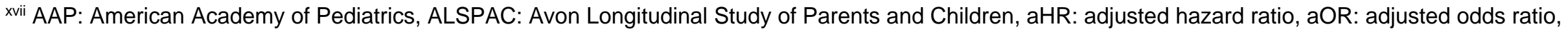

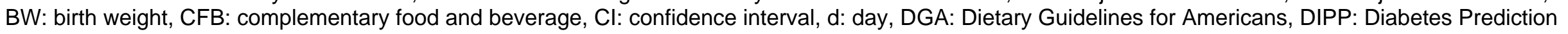

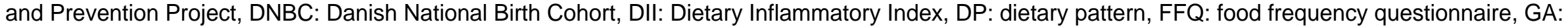
gestational age, HEl: Healthy Eating Index, HMF: human milk feeding, IQR: interquartile range, ISAAC: International Study of Asthma and Allergies in Childhood, mo: month(s), MUFA: monounsaturated fatty acid, NR: not reported, NS: non-significant, OFCs: Other factors considered, PCA: principal

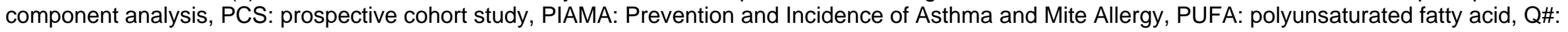

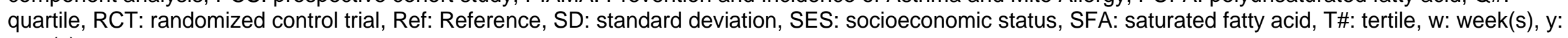
year(s) 


\section{Study and Participant}

Characteristics

- A level+: 35.4\%

- Housing tenure:

- Owned/mortgaged:

$73.8 \%$

- Council rented: $12.1 \%$

- Non-council rented:

$8.0 \%$

Unknown/other: 6.1\%

- Financial difficulties:

- None: $35.8 \%$

- Some: $38.0 \%$

- Many: $25.7 \%$

- Unknown: 0.5\%

- Characteristics reported with significant differences

by exposure

- Smoking

- HMF

- Characteristics reported but no differences by exposure

- Family history

○ GA

○ BW

Animal/pets/farming exposure
Intervention/Exposure and Outcomes

Results

- Vegetarian pattern: High consumption

of meat substitutes, pulses, nuts, and

herbal tea. Low consumption of

poultry and red meat

at 32wk gestation

\section{Dietary assessment methods:}

43-item FFQ at 32 wk gestation, based on FFQ validated in a British population. 5 dietary patterns were identified in the cohort using PCA: "health conscious", "traditional", "processed", "vegetarian" and "confectionery". DP scores were expressed in standard deviation units. Each mother was represented in each of these 5 mutually independent scores.

\section{Outcome:}

Asthma

\section{Outcome assessment methods:}

Children were defined as having current doctor-diagnosed asthma at $7.5 \mathrm{y}$ (primary outcome of interest) if mothers responded positively to the question: "Has a doctor ever actually said that your study child has asthma?" and positively to one or both of the questions on wheezing and asthma in the past $12 \mathrm{mo}$

$\begin{array}{ll}\text { Bédard, } 2018^{2} \text {; U.K. } & \text { Food(s) or Food Group(s): } \\ \text { PCS: ALSPAC } & \text { Weekly intake of fresh fruits by quartile }\end{array}$

Weekly intake of fresh fruits by quartile

\section{Significant:}

Non-significant:
Confounding, Study Limitations, and Summary of Findings

unlikely to have removed the risk of bias arising

from the missing data

- Majority of outcomes were subjective and the outcomes were assessed by participants, who were aware of the exposure received

- No data analysis plan available

\section{Summary:}

Adherence to dietary patterns during pregnancy was not associated with childhood asthma at 7.5y. 


\section{Study and Participant}

Characteristics

Baseline $\mathrm{N}=8,915$ Analytic

$\mathrm{N}=7,677$

- Age: 28.9y

- Race/Ethnicity:

oWhite: 98.2\%

- SES:

o Mother's educational level:

- Certificate of Secondary

Education: 15.4\%

- Vocational: $9.0 \%$

- Ordinary level: 35.5\%

- Advanced level: 25.1\%

- Degree: $15.1 \%$

o Housing tenure:

Owned/mortgaged:

83.7\%

o Financial difficulties:

$\sim 17.1 \%$

- Baseline characteristics

NR by exposure

\section{Intervention/Exposure and Outcomes Results}

\section{Dietary assessment:}

Data on maternal diet in pregnancy were collected by a FFQ covering all the main foods consumed in Britain.

The questionnaire included questions about the weekly frequency of consumption of 43 food groups and food items, with the possibility for respondents to tick one of the following options: never or rarely, once in 2 weeks, 1-3 times a week, 4-7 times a week, or more than once a day. One question on the weekly frequency of fresh fruit consumption and six questions on the weekly frequency of vegetables (peas, sweetcorn, broad beans; cabbage, brussels sprouts, kale and other green leafy vegetables; other green vegetables; carrots; other root vegetables; salad) were used to estimate weekly intake of fruits and vegetables, respectively, using standard portions.

\section{Outcome:}

Self-reported asthma at 7.5 y as positive response to the question "Has a doctor ever actually said that your study child has asthma?" and to one or both of the questions "Has your child had any of the following in the past 12 months: wheezing with whistling; asthma?"
Fresh fruit intake $(n=7,677)$

Q2-Q4 vs Q1 (Ref): $\mathrm{P}=\mathrm{NS}$

$P$ for trend $=0.26$

Confounding, Study Limitations, and Summary of Findings

OFCs accounted for:

Child Sex

Stratified by smoking status:

- Non/passive smokers: $\mathrm{P}$ for trend $=0.81$

- Active smokers: $P$ for trend=0.16

$P$ for interaction $=0.59$

Vegetable intake $(n=6,117)$

Q2-Q4 vs Q1 (Ref): $P=N S$

$P$ for trend $=0.22$

Stratified by smoking status:

- Non/passive smokers: P for trend=0.91

- Active smokers (OR): 0.88 (95\% $\mathrm{Cl}: 0.78,1.00)$

$\mathrm{P}$ for trend $=0.05$

$P$ for interaction $=0.18$

\section{Limitations:}

- At least one key confounder was not controlled for

- FFQ was not formally calibrated against other instruments, but was based on one which has been validated

- Potential for selection bias as the participants included in the study were more likely to human milk feed, have higher education, less likely to have tobacco exposure, anxiety in pregnancy. Included participants are also more likely to be White.

- Important co-exposures were not balanced across groups that were likely to impact the outcome, and no or inappropriate measurement and/or adjustment techniques were used to correct for the issues

- Majority of outcomes were subjective and the outcomes were assessed by participants, who were aware of the exposure received

- No data analysis plan available

\section{Summary:}

Maternal vegetable and fresh fruit intake during pregnancy was not associated with risk of childhood asthma at $7.5 \mathrm{y}$. 


\section{Study and Participan \\ Characteristics}

Bédard, 20201; U.K.

PCS: ALSPAC

Baseline $\mathrm{N}=8,907$ Analytic $\mathrm{N}=7,705$

- Age: 28.9y

- Race/Ethnicity:

o White: 98.2\%

o Non-White: $1.8 \%$

- SES:

- Mother's educational

level:

- Certificate of Secondary

Education: 15.4\%

- Vocational: 9.0\%

- Ordinary level: 35.5\%

- Advanced level: 25.1\%

- Degree: $15.1 \%$

o Housing tenure:

Owned/mortgaged: $\sim 83.7 \%$

o Financial difficulties:

$\sim 17.1 \%$

- Characteristics reported with significant differences by exposure

- Maternal age

- Maternal educational level

○ Smoking

o BW

- HMF duration

- Characteristics reported but no differences by exposure

\section{Intervention/Exposure and Outcomes Results}

\section{Food(s) or Food Group(s):}

Mediterranean Diet Score adapted for pregnant women

- MD score 0-3: Lower adherence to a Mediterranean-style diet $(n=3,475)$

- MD score 4-7: Higher adherence to a Mediterranean-style diet $(n=5,432$ Score based on the median weekly intake of 6 beneficial food groups (vegetables, legumes, fruits and nuts, cereal, fish and dairy) and 1 detrimental food group (meat).

at $32 \mathrm{wk}$ gestation

\section{Dietary assessment methods:}

43-item FFQ at 32 wk gestation, based on one validated in a British population. Women whose consumption of beneficial food groups was above the median were assigned a value of 1 , and those below were assigned a value of 0 . For the detrimental food group, consumption below the median was assigned a value of 1 , and above the median was assigned a value of 0 .

Food group values were summed together for a total ranging from 0 to 7 , with a higher score representing greater adherence to a Mediterranean-style diet.

Outcome: Asthma at $7.5 \mathrm{y}$

\section{Outcome assessment methods:}

Confounding, Study Limitations, and Summary of Findings

\section{Key confounders accounted for:}

Age, Race/ethnicity, SES, Smoking, Family history, HMF

\section{Non-significant:}

Asthma $(\mathrm{n}=7,634)$

- MD score 4-7 vs 0-3: $P=N S$

- Per unit increase: $\mathrm{P}=\mathrm{NS}$

\section{Limitations:}

- At least one key confounder was not controlled for

- FFQ was not formally calibrated against other instruments, but was based on one which has been validated

- Potential for selection bias as the participants included in the study were more likely to feed human milk, have higher education, less likely to have tobacco exposure, anxiety in pregnancy. Included participants are also more likely to be White.

- Important co-exposures imbalanced across groups that were likely to impact the outcome, and no adjustment techniques used to correct at least some of those variables

- Outcome measurement was subjective and were assessed by participants, who were aware of the exposure received

- Pre-registered data analysis NR

\section{Summary:}

Adherence to a Mediterranean-style diet during pregnancy was not associated with asthma, in the child at $7.5 \mathrm{y}$. 


\section{Study and Participant}

Characteristics

o Family history

○ GA

o Race/ethnicity

\section{Intervention/Exposure and Outcomes Results}

Asthma defined in children at $7.5 \mathrm{y}$ if

mothers responded positively to the

question "Has a doctor ever actually

said that your study child has asthma?"

and to one or both of the questions

"Has your child had any of the following

in the past 12 months: wheezing with

whistling; asthma?"

\section{Bunyavanich, 2014 ${ }^{4}$; U.S. PCS, Project Viva}

Baseline $\mathrm{N}=2,128$ Analytic $\mathrm{N}=1,277$

- Age: 32.3 (from other Project Viva data)

- Race/Ethnicity: White: 69\%

- SES:

o Maternal Education:

$\circ \geq$ college graduate: $69.3 \%$

o Household income $\geq \$ 70 \mathrm{~K}$

$63.0 \%$

- Reported but not tested by

exposure: Family history

\section{Food(s) or Food Group(s):}

Maternal consumption of major food allergens (servings/d z scores)

- Peanut:

$\circ 1^{\text {st }}$ trimester: $0.34 \pm 0.44$

$\circ 2^{\text {nd }}$ trimester: $0.36+0.43$

- Milk:

o $1^{\text {st }}$ trimester: $1.16 \pm 1.04$

$\circ 2^{\text {nd }}$ trimester: $1.50+1.82$

- Wheat:

$\circ 1^{\text {st }}$ trimester: $2.65+1.48$

$\circ 2^{\text {nd }}$ trimester: $2.69 \pm 1.44$

- Egg:

$\circ 1^{\text {st }}$ trimester: $0.32 \pm 0.30$

$\circ 2^{\text {nd }}$ trimester: $0.33 \pm 0.30$

- Soy:

$\circ 1^{\text {st }}$ trimester: $0.08 \pm 0.27$

$\circ 2^{\text {nd }}$ trimester: $0.08 \pm 0.28$

at $10 w k$ and $26-28$ wk gestation

\section{Dietary assessment methods:}

Maternal dietary assessments at the first and second trimester visits were based on a validated 166 -item semiquantitative $F F Q$ modified for pregnancy. The total servings per day of each major food allergen (peanut,
Confounding, Study Limitations, and Summary of Findings

\section{Significant:}

Maternal 1st trimester milk intake (per z score):

$\mathrm{aOR}=0.83,95 \% \mathrm{Cl}$ (0.69, 0.99)

\section{Non-significant:}

Maternal 1st trimester intake of peanut, wheat, egg, or soy, $\mathrm{P}=\mathrm{NS}$

Maternal 2nd trimester intake of any food allergens, $\mathrm{P}=\mathrm{NS}$

Stratification by parental atopy showed no associated between intake of any food allergens during 1 st or 2nd trimester and asthma.

\section{Key confounders accounted for:}

(Race/Ethnicity adjusted in a secondary model), SES, Family history, HMF

\section{OFCs accounted for:}

Child sex

\section{Limitations:}

- Critical co-exposures NR

- Mothers who participated in the study were different than those who were lost to follow-up on the following characteristics: maternal race, college education, income, parental atopy

- Proportions of and reasons for missingness NR by exposure

- Multiple exposure outcome comparisons were assessed without using an appropriate p-value correction

- Self-reported exposure and outcome (for clinical symptoms)

- Pre-registered data analysis plan NR

\section{Summary:}

Higher maternal consumption of milk during the 1st trimester of pregnancy was associated with reduced risk of asthma in the child at $7.9 \mathrm{y}$. 


\begin{tabular}{|c|c|c|c|}
\hline $\begin{array}{l}\text { Study and Participant } \\
\text { Characteristics }\end{array}$ & Intervention/Exposure and Outcomes & Results & $\begin{array}{l}\text { Confounding, Study Limitations, and Summary } \\
\text { of Findings }\end{array}$ \\
\hline & $\begin{array}{l}\text { milk, wheat, egg, and soy) were } \\
\text { calculated by summing the servings per } \\
\text { day of the foods on the FFQ containing } \\
\text { these respective food allergens. } \\
\text { Outcome: } \\
\text { Asthma at } 7.9 \text { y } \\
\text { Outcome assessment methods: } \\
\text { Current asthma was defined as positive } \\
\text { if a mother reported at the mid- } \\
\text { childhood visit that her child ever had } \\
\text { doctor-diagnosed asthma plus either } \\
\text { use of asthma medication or wheezing } \\
\text { in the past } 12 \text { mo. Ever asthma was } \\
\text { defined as positive if a mother reported } \\
\text { a doctor's diagnosis of asthma in the } \\
\text { child in any questionnaire since birth. }\end{array}$ & & $\begin{array}{l}\text { Maternal intake of peanut, wheat, egg, or soy in the } \\
\text { 1st trimester or of any major food allergens in the } \\
\text { 2nd trimester were not associated with risk of } \\
\text { asthma in the child at } 7.9 \mathrm{y} \text {. }\end{array}$ \\
\hline Wright, 2018 ${ }^{33}$; U.S. & Food(s) or Food Group(s): & Significant: & Key confounders accounted for: \\
\hline $\begin{array}{l}\text { PCS, Project Viva } \\
\text { Baseline } \mathrm{N}=1,068 \text { Analytic } \\
\mathrm{N}=1053 \\
\text { - Age: } 32.5 \pm 5.0 \mathrm{y} \\
\text { - Race/Ethnicity (child): } \\
\text { - White: } 68 \% \\
\text { - SES: } \\
\text { - College grad or higher: } \\
71 \% \\
\text { - Household income }>\$ 70 \mathrm{~K} \text { : } \\
62 \%\end{array}$ & $\begin{array}{l}\text { Mean of } 1 \text { st and } 2 \text { nd trimester sugar- } \\
\text { sweetened beverages (regular soda } \\
\text { and fruit drinks) intake by quartile and } \\
\text { continuous intake (servings/d) } \\
\text { Q1: } n=283 \\
\text { Q2: } n=251 \\
\text { Q3: } n=260 \\
\text { Q4: } n=259 \\
\text { Mean of } 1 \text { st and } 2 \text { nd trimester fruit juice } \\
\text { intake by quartile and continuous intake } \\
\text { (servings/d) } \\
\text { at } \sim 11.9 \text { wk and } \sim 29.2 \text { wk gestation }\end{array}$ & $\begin{array}{l}\text { Sugar-sweetened beverages } \\
\text { Q4 vs Q1: aOR=1.70, } 95 \% \mathrm{Cl} \text { : }(1.08 \text {, } \\
2.67) \\
\text { Non-Significant: } \\
\text { Sugar-sweetened beverages } \\
\text { Q2-Q3 vs } \mathrm{Q} 1, \mathrm{P}=\mathrm{NS} \\
\text { Continuous intake, } \mathrm{P}=\mathrm{NS} \\
\text { Juice intake (continuous or by } \\
\text { quartile), } \mathrm{P}=\mathrm{NS}\end{array}$ & $\begin{array}{l}\text { Race/ethnicity (child), SES, Smoking, Family history } \\
\text { OFCs accounted for: } \\
\text { Child Sex } \\
\text { Limitations: } \\
\text { - Not all confounders accounted for } \\
\text { - Potential selection bias, as those included in the } \\
\text { study were slightly older (mean, } 32.5 \text { vs } 31.1 \mathrm{y}) \text {, } \\
\text { more likely to have graduated from college }(71 \% \\
\text { vs } 58 \% \text { ), have household income exceeding } \\
\$ 70,000 \text { compared to those who were not included } \\
\text { - Adjusted for post-exposure variables }\end{array}$ \\
\hline
\end{tabular}




\section{Study and Participant}

Characteristics

- Age, SES, and Smoking in pregnancy associated with sugar-sweetened beverage intake

\section{Intervention/Exposure and Outcomes Results}

\section{Dietary assessment:}

Data on consumption of beverages during pregnancy from semiquantitative FFQ was obtained from expectant mothers who completed it after the first and second research visits. Participants endorsed categories of frequency of beverage consumption from "never/less than one per month" to a maximum of "two or more glasses per day" for some fruit juices, "four or more cans per day" for soda, and "six or more glasses per day" for water.

\section{Outcomes:}

Current asthma defined as self-reported ever doctor-diagnosed asthma at $\sim 7.7 \mathrm{y}$ plus wheezing symptoms or asthma medications in the past year.

Comparison group had no asthma diagnosis ever and no wheezing or asthma medication use in the past 12 mo.

Hanson, $2020^{9}$; U.S. PCS, Project Viva

Baseline $\mathrm{N}=1,424$

Analytic $\mathrm{N}=1,424$

- Age: $32.3 \pm 5.0 y$

- Race/Ethnicity:

oWhite: $71 \%$

- SES:

○College grad: $70 \%$

\section{Food(s) or Food Group(s):}

$1^{\text {st }}$ trimester, 2 nd trimester, and average of $1^{\text {st }}$ and $2^{\text {nd }}$ trimester. Dietary Inflammatory Index (DII) by quartile Q1: $n=362$

Q2: $n=358$

Q3: $n=362$

\section{Q4: $\mathrm{n}=342$}

Higher quartiles of DII represent a more proinflammatory diet relative to lower quartiles

at $\sim 9.9$ wk and $\sim 29.9$ wk gestation
Confounding, Study Limitations, and Summary of Findings

- Proportions of and reasons for missingness NR by exposure

- Self-reported, doctor-diagnosed outcomes

- Pre-registered data analysis plan NR

\section{Summary:}

High maternal consumption of sugar-sweetened beverages averaged across the 1st and 2nd trimester of pregnancy was associated with increased odds of asthma in the child at $7.7 \mathrm{y}$, compared to low consumption.

Lower levels of sugar-sweetened beverage intake and any level of juice intake during pregnancy was not associated with the odds of asthma in the child at $7.7 \mathrm{y}$.

\section{Significant:}

\section{Non-significant:}

No association between DII in 1st trimester, 2nd trimester, or average of $1 \mathrm{st}$ and 2 nd trimesters and ever or current asthma in the child at 3.3y or $7.7 y$

\section{Key confounders accounted for:}

Race/ethnicity, SES,

Smoking, Family history

\section{OFCs accounted for:}

Child Sex

\section{Limitations:}

- Not all confounders accounted for 50\% attrition; - Self-reported, doctor-diagnosed outcomes 


\section{Study and Participant}

Characteristics

Intervention/Exposure and Outcomes

Results

o Household income $>\$ 70 \mathrm{~K}$

$64 \%$

- Reported with no

differences by exposure:

Smoking, Family history

\section{Dietary assessment:}

The FFQ assessed dietary intake since the last menstrual period (first-trimester FFQ) or during the previous 3 mo (second-trimester FFQ). Resulting dietary data was used to calculate DII scores for each

Participant. The 28 dietary parameters used for the DII calculation were energy,

carbohydrate, protein, fat, alcohol, fiber, cholesterol, saturated fatty acids, monounsaturated fatty acids, polyunsaturated fatty acids, omega3 and omega- 6 fatty acids, trans-fatty acids, niacin, thiamine, riboflavin, vitamin $\mathrm{B} 12$, vitamin $\mathrm{B} 6$, iron,

magnesium, zinc, selenium, vitamin $A$, vitamin $C$, vitamin $D$, vitamin $E$, folic acid, and b-carotene

\section{Outcomes:}

Self-reported ever doctor-diagnosed asthma at $~ 3.3 y$ and $~ 7.7 y$ (mid childhood)

\section{Outcome assessment methods:}

For ever doctor-diagnosed asthma, the investigators asked the parent: "Have you ever been told by a health care professional, such as a doctor, physician assistant, or nurse practitioner, that your child has asthma?"
Confounding, Study Limitations, and Summary of Findings

- Potential for selection bias, as those included in the study were slightly older (mean, 32.3 vs 30.8 years), more likely to have graduated from college (70\% vs $52 \%$ ), and White ( $71 \%$ vs $56 \%$ ) compared to those who were not included

- Pre-registered data analysis plan NR

\section{Summary:}

Maternal dietary inflammatory index during the 1 st and/or 2nd trimester of pregnancy was not associated with risk of asthma in the child at $3.3 \mathrm{y}$ or $7.7 \mathrm{y}$. 
Mid-childhood current asthma was defined as ever doctor-diagnosed asthma plus wheezing symptoms or asthma medications in the past year. Participants with never asthma diagnosis, no wheezing, and no asthma medicines in the past year were used as the comparison group.

\section{Maslova, 2012 ${ }^{17}$; Denmark PCS, DNBC}

Baseline $\mathrm{N}=61908$ Analytic $\mathrm{N}=38570$

- Age: $29.0 \pm 4.0 y$

- Race/Ethnicity: NR

- SES:

○ SES Position: High-level proficiencies: $23 \%$

- Characteristics reported by exposure but unknown significance

- Smoking

○ Family history

$\circ \mathrm{GA}$

$\circ B W$

○HMF

\section{Food(s) or Food Group(s):}

Peanut and pistachio intake

Tree nut intake

- Group 1: Never

- Group 2: 1 time/mo

- Group 3: 1-3 times/mo

- Group 4: $\geq 1$ time/wk

\section{Dietary assessment methods}

Peanut and nut intake was assessed during mid-pregnancy by using a

validated 360 -item semi-quantitative FFQ15 that covered intake in the past 4wk. Specific questions were asked about snack consumption in the past month, separately assessing "peanut and pistachio" intake and the intake of

"nuts and almonds." We assumed that most women consumed peanuts rather than pistachios in the "peanut and pistachio" category.

once at 25 wk gestation

\section{Outcomes:}

\section{Significant:}

Ever admitted asthma

Peanut and pistachio intake

$(\mathrm{n}=38,570), \mathrm{P}$ for trend $=0.002$

- Group 2 vs Group 1 (Ref): P=NS

- Group 3 vs Group 1 (Ref): aOR $=0.82,95 \% \mathrm{Cl}=(0.69,0.97)$

- Group 4 vs Group 1 (Ref): $\mathrm{aOR}=0.66,95 \% \mathrm{Cl}=(0.44,0.98)$

Ever prescribed asthma

Tree nut intake $(n=38,494), P$ for trend $=0.0003$

- Group 2 vs Group 1 (Ref):

$\mathrm{aOR}=0.91,95 \% \mathrm{Cl}=(0.84,0.98)$

- Group 3 vs Group 1 (Ref): P=NS

- Group 4 vs Group 1 (Ref):

$\mathrm{aOR}=0.81,95 \% \mathrm{Cl}=(0.73,0.90)$

\section{Non-significant:}

Current asthma at $7 \mathrm{y}$

Peanut and pistachio intake

$(n=13,443), P$ for trend $=0.27$

\section{Key confounders accounted for:}

Age, SES, Smoking, Family history, HMF

\section{OFCs accounted for:}

None

\section{Limitations:}

- Total sample size in the text and table does not match. It is unclear why additional participants were dropped from the study

- No differentiation between peanut and pistachio intake

- Exposure does not include processed nut products

- At least one key confounder not adjusted for

- No information on deviation from intended exposures

- Critical co-exposures not accounted for in the analysis

- Self-reported outcome measure only minimally influenced by knowledge of the exposure received by study participants

- Potential selection bias, as participants that completed the study were significantly different than the non-participants on the following characteristics: SES, parity, and smoking status 
Study and Participant

Characteristics

\section{Intervention/Exposure and Outcomes Results}

Current asthma at $7 y$ defined as self-

reported doctor diagnosis of asthma

plus wheeze in the past $12 \mathrm{mo}$.

\section{Outcome assessment methods:}

Ever admitted asthma defined as first registered asthma diagnosis in the International Classifications of Disease.

Ever prescribed asthma defined as $\geq 2$ asthma prescriptions in the Register of Medicinal Product Statistics (RMPS) except for $b-2$ agonists as liquid, inhaled b-2 agonists only once or inhaled steroid only once. $\mathrm{P}=\mathrm{NS}$ $\mathrm{P}=\mathrm{NS}$ $\mathrm{P}=\mathrm{NS}$ Danish National Patient Registry using trend $=0.19$

\section{Maslova, $2012^{18}$; Denmark PCS, DNBC}

Baseline $\mathrm{N}=61909$

Analytic $\mathrm{N}=39058$

- Age: $21-39 y: 98.0 \%$

- Race/Ethnicity: NR

- SES:

o SES Position: High-level proficiencies: $23 \%$

\section{Food(s) or Food Group(s):}

Total dairy product intake and total milk intake by glasses/d:

- Group 1: >0

- Group 2: >1-2

- Group 3: >2-3

- Group 4: >3-4

- Group 5: >4-5

- Group 6: >5

Whole milk intake and semi-skimmed milk intake by frequency of consumption:
- Groups 2-4 vs Group 1 (Ref):

Tree nut intake $(n=13,415), P$ for trend $=0.99$

- Groups 2-4 vs Group 1 (Ref):

Ever admitted asthma

Tree nut intake $(n=38,512), P$ for

- Groups 2-4 vs Group 1 (Ref):

Ever prescribed asthma

Peanut and pistachio intake $(\mathrm{n}=38,552), \mathrm{P}$ for trend $=0.29$

- Groups 2-4 vs Group 1 (Ref): $\mathrm{P}=\mathrm{NS}$

Confounding, Study Limitations, and Summary of Findings

- No pre-registered data analysis plan

\section{Summary:}

Higher maternal peanut and pistachio intake during pregnancy was associated with a reduced risk of ever admitted asthma in childhood, compared with lower intake.

Higher maternal tree nut intake during pregnancy was associated with a reduced risk of ever prescribed asthma medication in childhood, compared with lower intake.

Maternal peanut and pistachio intake, as well as tree nut intake, during pregnancy was not associated with current childhood asthma at 7 years.

\section{Significant:}

Ever admitted asthma

- Low-fat yogurt intake ( $n=37,871), P$ for trend $=0.03$

- Group 2 vs Group 1 (Ref): P=NS

- Group 3 vs Group 1 (Ref): $\mathrm{aOR}=1.20,95 \% \mathrm{Cl}=(1.01,1.42)$

- Group 4 vs Group 1 (Ref): P=NS

Ever prescribed asthma:

- Full-fat yogurt intake $(n=39,002), P$ for trend $=0.01$

\section{Key confounders accounted for:}

Age, SES, Smoking, Family history, HMF

\section{OFCs accounted for:}

Child sex

\section{Limitations:}

- At least one key confounder not controlled for

- Selection into the study was related (but not very strongly) to exposure and outcome and this could not be adjusted for in analyses; 


\section{Study and Participant}

Characteristics

- Characteristics reported by
exposure but unknown
significance
o Smoking ( 25\% with $13 \%$
current smokers)
$\circ$ Family history
$\circ$ GA
$\circ$ BW
$\circ$ HMF

\section{Intervention/Exposure and Outcomes Results}

o Group 2 vs Group 1 (Ref):

aOR $=0.87,95 \% \mathrm{Cl}=(0.81,0.93)$

- Groups 3-4 vs Group 1 (Ref): $\mathrm{P}=\mathrm{NS}$

\section{Non-Significant:}

Full-fat yogurt intake and low-fat yogurt intake by servings/d:

- Group 1:0

- Group 2: >0-0.5

- Group 3: $>0.5-1$

- Group 4: >1

Dietary assessment: A validated 360 item semi-quantitative FFQ was completed around gestation week 25; it referred to intake during the previous 4 weeks. Dairy product consumption was recorded in 8 questions in the FFQ; two of them asked about consumption of yogurt, in servings per day (including percentage of fat, with/without fruit) and 6 questions asked about milk consumption (whole milk, $1.5 \%$ milk, $0.5 \%$ milk, skimmed milk, churn buttermilk and chocolate milk) in glasses per day. The FFQ asked about yogurt with/without fruit to better estimate carbohydrates. Assuming that the serving sizes were approximately equal to $200 \mathrm{ml}$, the milk and yogurt variables were aggregated to obtain the frequency measures of total dairy intake. Frequency of milk intake was quantified by summing all types of milk and excluding yogurt. For our analyses,

$(n=13,635)$, $P$ for trend $=0.12$

o Group 2 vs Group 1 (Ref): $\mathrm{aOR}=0.79,95 \% \mathrm{Cl}=(0.62,0.99)$ $0.73,95 \% \mathrm{Cl}=(0.58,0.92)$

- Groups 5-6 vs Group 1 (Ref): $\mathrm{P}=\mathrm{NS}$ trend $=0.10$

- Groups 2-6 vs Group 1 (Ref): $\mathrm{P}=\mathrm{NS}$ trend $=0.63$

- Groups 2-5 vs Group 1 (Ref): $\mathrm{P}=\mathrm{NS}$

- Semi-skimmed milk intake $(n=13,285), P$ for trend=0.13

o Group 2 vs Group 1 (Ref): aOR $=0.48,95 \% \mathrm{Cl}=(0.26,0.88)$

- Groups 3-5 vs Group 1 (Ref): $\mathrm{P}=\mathrm{NS}$ for trend $=0.20$
Confounding, Study Limitations, and Summary of Findings

- No information on deviation from intended exposures;

- Self-reported outcome measure only minimally influenced by knowledge of the exposure received by study participants;

- Potential selection bias, as participants that completed the study were significantly different than the non-participants on the following characteristics: SES, parity, and smoking status

- Critical co-exposures not accounted for in the analysis

- Group 3 vs Group 1 (Ref): P=NS - Group 4 vs Group 1 (Ref): aOR=

- Total milk intake $(n=13,600), P$ for

- Whole milk intake $(n=13,324), P$ fo

- Full-fat yogurt intake $(n=13,620), P$
- Possible selective reporting of the findings

- No pre-registered data analysis plan

\section{Summary:}

Higher maternal low-fat yoghurt intake during pregnancy was associated with greater risk for ever admitted asthma, compared to lower intake.

Higher maternal full-fat yoghurt intake during pregnancy was associated with reduced risk for ever prescribed asthma, compared to lower intake.

Maternal total dairy product and milk intake during pregnancy was not associated with risk of childhood asthma. 
Study and Participant

Characteristics

\section{Intervention/Exposure and Outcomes Results}

o Groups 2-4 vs Group 1 (Ref):

$$
\mathrm{P}=\mathrm{NS} \text {; }
$$

as total dairy product, total milk and

total full-fat and low-fat yoghurt intake was examined.

Once at 25wk gestation

\section{Outcomes:}

Current asthma at $7 y$ defined as selfreported doctor diagnosis of asthma plus wheeze in the past $12 \mathrm{mo}$.

Ever admitted asthma defined as first registered asthma diagnosis in the Danish National Patient Registry using International Classifications of Disease.

Ever prescribed asthma defined as $\geq 2$ asthma prescriptions in the Register of Medicinal Product Statistics (RMPS) except for b-2 agonists as liquid, inhaled b-2 agonists only once or inhaled steroid only once.

- Low-fat yogurt intake $(n=13,182), P$

for trend=0.06

- Groups 2-4 vs Group 1 (Ref):

$\mathrm{P}=\mathrm{NS}$

Ever admitted asthma:

- Total dairy product intake $(n=39,058), P$ for trend $=0.56$ o Groups 2-6 vs Group 1 (Ref): $\mathrm{P}=\mathrm{NS}$

- Total milk intake $(n=38,971), P$ for trend $=0.68$

o Groups 2-6 vs Group 1 (Ref): $\mathrm{P}=\mathrm{NS}$

- Whole milk intake $(n=38,285), P$ for trend $=0.08$

- Group 2 vs Group 1 (Ref):
Confounding, Study Limitations, and Summary

of Findings

$\mathrm{aOR}=1.45,95 \% \mathrm{Cl}=(1.05,1.99)$

- Groups 3-5 vs Group 1 (Ref):

$\mathrm{P}=\mathrm{NS}$

- Semi-skimmed milk intake $(n=38,175), P$ for trend $=0.69$

o Groups 2-5 vs Group 1 (Ref): $\mathrm{P}=\mathrm{NS}$

- Full-fat yogurt intake $(n=39,020), P$ for trend $=0.48$ 
Study and Participant

Characteristics
Intervention/Exposure and Outcomes Results

o Groups 2-4 vs Group 1 (Ref):

$\mathrm{P}=\mathrm{NS}$

Ever prescribed asthma:

- Total dairy product intake

$(n=39,040), P$ for trend $=0.46$

Groups 2-6 vs Group 1 (Ref):

$\mathrm{P}=\mathrm{NS}$

- Total milk intake $(n=38,953), P$ for trend $=0.26$

- Groups 2-5 vs Group 1 (Ref):

$\mathrm{P}=\mathrm{NS}$

- Group 6 vs Group 1 (Ref): $\mathrm{aOR}=1.13,95 \% \mathrm{Cl}=(1.02,1.26)$

- Whole milk intake $(n=38,267), P$ for trend $=0.33$

- Group 2 vs Group 1 (Ref): P=NS

- Group 3 vs Group 1 (Ref): aOR $=0.81,95 \% \mathrm{Cl}=(0.68,0.95)$

- Groups 4-5 vs Group 1 (Ref):

$\mathrm{P}=\mathrm{NS}$

- Semi-skimmed milk intake

$(n=38,158), P$ for trend $=0.19$

- Groups 2-5 vs Group 1 (Ref):

$\mathrm{P}=\mathrm{NS}$

- Low-fat yogurt intake $(n=37,853), P$

for trend $=0.15$

Group 2 vs Group 1 (Ref):

$\mathrm{aOR}=1.07,95 \% \mathrm{Cl}=(1.01,1.14)$

- Group 3 vs Group 1 (Ref): P=NS

Groups 4 vs Group 1 (Ref):

$\mathrm{aOR}=1.21,95 \% \mathrm{Cl}=(1.02,1.45)$
Confounding, Study Limitations, and Summary

of Findings 
Study and Participant

Characteristics

Maslova, 2013 ${ }^{19}$; Denmark

PCS, DNBC

Baseline $\mathrm{N}=28,936$ Analytic $\mathrm{N}=16,867$

- Age:

o $\leq 20 \mathrm{y}: 0.7 \%$

o $\geq 40 y: 1.2 \%$

- Race/Ethnicity: NR

- SES:

- SES Position:

- High-level proficiencies: $22.9 \%$

- Medium-level

proficiencies: $29.4 \%$

- Skilled: $24.5 \%$

- Unskilled: $10.6 \%$

- Students: $4.0 \%$

- Unemployed: $2.5 \%$

- Missing: 6.1\%

- Characteristics with significant differences by exposure

o Smoking

oBW

o HMF

- Characteristics with no differences by exposure

o Family history

$\circ \mathrm{GA}$

\section{Intervention/Exposure and Outcomes Results}

\section{Significant:}

None

Fish intake

- Group 1: Never eating fish ( 11\%)

- Group 2: Hot meal and sandwiches $\leq 1 / \mathrm{mo}(\sim 25 \%)$

- Group 3: Hot meal 1/mo, sandwiches 1/wk ( 37\%)

- Group 4: Hot meals 1/wk, sandwiches 1-2x/wk ( 23\%)

- Group 5: Hot meals $>2 / w k$, sandwiches $>3 \mathrm{x} / \mathrm{wk}(\sim 4 \%)$

At 12 and 30 wk (telephone interview that assessed fish intake with a sandwich or a hot meal)

FFQ at 25 weeks also assessed fish intake in $\mathrm{g} / \mathrm{wk}$

\section{Dietary assessment:}

The FFQ asked about intake in the past 4 weeks and has been validated against $7 \mathrm{~d}$ food diaries and blood and urine biomarkers for selected nutrient (protein, retinol, folic acid and n-3 PUFA) and food (fruit and vegetable) intake.

\section{Outcomes:}

Current asthma at $7 \mathrm{y}$ defined as selfreported doctor diagnosis of asthma plus wheeze in the past $12 \mathrm{mo}$.

Confounding, Study Limitations, and Summary of Findings

\section{Key confounders accounted for:}

Age, SES, Smoking, Family history, HMF

\section{Non-significant:}

Current asthma at $7 y$

Fish intake $(n=11,586), P$ for trend $=0.75$

- Groups $1-4$ vs Group 5 (Ref):
P=NS

Ever admitted asthma

Fish intake $(n=11,631), P$ for trend $=0.46$

- Groups 1-4 vs Group 5 (Ref): $\mathrm{P}=\mathrm{NS}$

Ever prescribed asthma

Fish intake $(n=11,622), P$ for trend $=0.06$

- Groups 2-4 vs Group 5 (Ref): $\mathrm{P}=\mathrm{NS}$

- Group 1 vs Group 5: aOR=1.37, $95 \% \mathrm{Cl}:(1.10,1.71), \mathrm{P}=0.01$

\section{OFCs accounted for:}

Child sex

\section{Limitations:}

- At least one key confounder not adjusted for

- Selection into the analysis was related to exposure and may be related to outcome and this could not be adjusted for in the analyses

- Participants tended to display healthier lifestyle habits and a higher SES compared to nonparticipants

- Critical co-exposures not accounted for in the analysis

- Self-reported outcome measure only minimally influenced by knowledge of the exposure received by study participants

- No pre-registered data analysis plan

\section{Summary:}

Fish intake during pregnancy was not associated with risk of ever admitted asthma or current asthma at 7 years.

Although $\mathrm{P}$ for trend was not significant, no fish intake during pregnancy was associated with a higher risk of ever prescribed asthma compared with high fish intake. 
Ever admitted asthma defined as first

registered asthma diagnosis in the

Danish National Patient Registry using

International Classifications of Disease.

Ever prescribed asthma defined as $\geq 2$ asthma prescriptions in the Register of Medicinal Product Statistics (RMPS) except for b-2 agonists as liquid, inhaled b-2 agonists only once or inhaled steroid only once.

\section{Maslova, $2013^{20}$; Denmark PCS, DNBC}

Baseline $\mathrm{N}=60465$ Analytic $N=38398$

- Age:

o $\leq 20 \mathrm{y}: 1.0 \%$

o $\geq 40 y: 0.9 \%$

- Race/Ethnicity: NR

- SES:

- SES Position:

- High-level proficiencies:

$20.6 \%$

- Medium-level

proficiencies: $27.6 \%$

- Skilled: $24.1 \%$

- Unskilled: $11.3 \%$

- Students: 4.0\%

- Unemployed: $2.4 \%$

- Missing: 10.0\%

- Characteristics reported but no test for differences by exposure

\section{Significant:}

Ever prescribed asthma

Soft drink intake by frequency of intake

- Artificially-sweetened carbonated

- Group 1: Never: 67\%

- Group 2: 1 serving/wk: 13\%

o Group 3: 2-6 servings/wk: 16\%

- Group 4: $\geq 1$ serving/d: 4\%

- Artificially-sweetened non-carbonated

- Group 1: Never: 67\%

- Group 2: 1 serving/wk: 7\%

- Group 3: 2-6 servings/wk: $13 \%$

- Group 4: $\geq 1$ serving/d: 13\%

- Sugar-sweetened carbonated

- Group 1: Never: 16\%

o Group 2: 1 serving/wk: 26\%

- Group 3: 2-6 servings/wk: 48\%

o Group 4: $\geq 1$ serving/d: 10\%

- Sugar-sweetened non-carbonated

- Group 1: Never: 35\%

- Group 2: 1 serving/wk: 15\%

- Group 3: $2-6$ servings/wk: $28 \%$

o Group 4: $\geq 1$ serving/d: 22\%

assessed once at 25 wk gestation
- Artificially-sweetened carbonated soft drink intake $(n=38,258), P$ for trend $=0.01$

- Group 2 vs Group 1 (Ref): aOR $=1.11,95 \% \mathrm{Cl}:(1.02,1.20)$

- Group 3 vs Group 1 (Ref):

$\mathrm{aOR}=1.10,95 \% \mathrm{Cl}:(1.02,1.19)$

- Group 4 vs Group 1 (Ref): P=NS

- Artificially-sweetened noncarbonated soft drink intake

$(n=38,272), P$ for trend $=0.001$

o Groups 2-3 vs Group 1 (Ref):

$\mathrm{P}=\mathrm{NS}$

- Group 4 vs Group 1 (Ref):

aOR=1.15, 95\% Cl: $(1.06,1.25)$

\section{Non-significant:}

Current asthma at $7 y$

\section{Key confounders accounted for:}

Age, SES, Smoking, Family history, Delivery mode, HMF, Animals/pets/farming

\section{OFCs accounted for:}

Child sex

\section{Limitations:}

- At least one key confounder not controlled for

- Participants tended to display healthier life-style habits compared to non-participants

- No information on deviation from intended exposure

- Critical co-exposures not accounted for in the analysis

- Self-reported outcome measure only minimally influenced by knowledge of the exposure received by study participants

- No pre-registered data analysis plan

\section{Summary:}




\section{Study and Participant}

Characteristics

o Smoking

o Family history

$\circ \mathrm{GA}$

$\circ \mathrm{BW}$

o HMF

\section{Intervention/Exposure and Outcomes Results}

- Artificially-sweetened carbonated soft drink intake and $(n=38,149), P$ for trend $=0.27$

Maternal diet assessment was based on a validated 360 -item semi-

quantitative FFQ completed around gestation week 25 and

covered intake during the previous $4 \mathrm{wk}$

\section{Outcomes:}

Current asthma at $7 y$ defined as selfreported doctor diagnosis of asthma plus wheeze in the past $12 \mathrm{mo}$.

\section{Outcome assessment methods:}

Ever admitted asthma defined as first registered asthma diagnosis in the Danish National Patient Registry using International Classifications of Disease.

Ever prescribed asthma defined as $\geq 2$ asthma prescriptions in the Register of Medicinal Product Statistics (RMPS) except for b-2 agonists as liquid, inhaled b-2 agonists only once or inhaled steroid only once.

$$
\mathrm{P}=\mathrm{NS}
$$

- Similar results for artificiallysweetened non-carbonated soft drinks, sugar-sweetened carbonated soft drinks, and sugarsweetened non-carbonated soft drinks

Ever admitted asthma

- Artificially-sweetened carbonated soft drink intake $(n=38,258), P$ for trend $=0.19$

$$
\mathrm{P}=\mathrm{NS}
$$

- Group 4 vs Group 1 (Ref): aOR $=1.30,95 \% \mathrm{Cl}:(1.01,1.66)$

- Artificially-sweetened noncarbonated soft drink intake $(n=38,272), P$ for trend $=0.08$ - Groups 2-4 vs Group 1 (Ref):

\section{Confounding, Study Limitations, and Summary} of Findings

Higher maternal artificially-sweetened carbonated and non-carbonated soft drink intake during pregnancy was associated with a higher risk of ever prescribed asthma in childhood, compared with lower intake.

o Groups 2-3 vs Group 1 (Ref) $\mathrm{P}=\mathrm{NS}$

- Similar results for sugar-sweetened carbonated soft drinks and sugarsweetened non-carbonated soft drinks ( $p$ for trend $=0.05$ )

\section{Ever prescribed asthma}

- Sugar-sweetened carbonated soft drink intake $(n=38,398), P$ for trend $=0.72$
Although the $P$ for trend was not significant, high maternal artificially-sweetened carbonated soft drink intake during pregnancy was associated with a higher risk of ever admitted asthma, compared with no intake.

Sugar-sweetened soft drink consumption during pregnancy was not associated with risk of childhood asthma. 


\begin{tabular}{|c|c|c|c|}
\hline $\begin{array}{l}\text { Study and Participant } \\
\text { Characteristics }\end{array}$ & Intervention/Exposure and Outcomes & Results & $\begin{array}{l}\text { Confounding, Study Limitations, and Summary } \\
\text { of Findings }\end{array}$ \\
\hline & & $\begin{array}{l}\text { o Groups 2-4 vs Group 1 (Ref): } \\
\mathrm{P}=\mathrm{NS} \\
\text { - Similar results for sugar-sweetened } \\
\text { non-carbonated soft drinks ( } \mathrm{p} \text { for } \\
\text { trend }=0.07 \text { ) }\end{array}$ & \\
\hline Viljoen, $2018^{30}$; Ireland & Food(s) or Food Group(s): & Significant: & Key confounders accounted for: \\
\hline $\begin{array}{l}\text { PCS; Lifeways Cross- } \\
\text { Generation }\end{array}$ & $\begin{array}{l}\text { - Vegetable intake (servings/d) } \\
\text { - Oily fish intake (servings/d) }\end{array}$ & Non-Significant: & SES, Smoking, HMF practices, GA \\
\hline $\begin{array}{l}\text { Baseline } N=897 \text { Analytic } \\
N=682\end{array}$ & $\begin{array}{l}\text { assessed once during first trimester of } \\
\text { pregnancy }\end{array}$ & $\begin{array}{l}\text { Vegetable intake and risk of asthma } \\
\text { over } 10 \text { y follow-up period }(n=682) \\
P=N S\end{array}$ & $\begin{array}{l}\text { OFCs accounted for: } \\
\text { None }\end{array}$ \\
\hline & Outcomes: & Oily fish intake and risk of asthma & Limitations: \\
\hline $\begin{array}{l}\text { - Age at Delivery: }<18 \mathrm{y} \text { : } \\
\text { 1.8\%, } \geq 30 \mathrm{y}: 55.7 \% \\
\text { - Race/Ethnicity: } 100 \% \text { Irish- } \\
\text { born } \\
\text { - SES: } 51.9 \% \geq \text { tertiary } \\
\text { education, } 79.6 \% \\
\text { cohabiting, } 14.8 \% \text { eligible } \\
\text { for free healthcare }\end{array}$ & $\begin{array}{l}\text { Doctor-diagnosed asthma over a } 10 \text { y } \\
\text { follow-up period (assessed at ages } 3,5 \text {, } \\
\text { and } 9 \text { y) }\end{array}$ & $\begin{array}{l}\text { over } 10 \text { y follow-up period }(n=678) \\
P=N S\end{array}$ & $\begin{array}{l}\text { At least one key confounder not controlled for ; } \\
\text { Selection bias likely; Proportions of and reasons for } \\
\text { missing participants may differ across exposure } \\
\text { groups and the analysis is unlikely to have removed } \\
\text { the risk of bias arising from the missing data; No } \\
\text { pre-registered data analysis plan; Suspected } \\
\text { selective reporting }\end{array}$ \\
\hline & & & Summary: \\
\hline $\begin{array}{l}\circ \text { Age } \\
\circ \text { SES } \\
\circ \text { Smoking (for vegetable } \\
\text { intake) }\end{array}$ & & & $\begin{array}{l}\text { Maternal vegetable intake and oily fish intake during } \\
\text { pregnancy are not associated with risk of childhood } \\
\text { asthma over the first } 10 \text { years. }\end{array}$ \\
\hline Chen, 20206; Ireland & Food(s) or Food Group(s): & Significant: & Key confounders accounted for: \\
\hline \multirow{2}{*}{$\begin{array}{l}\text { PCS; Lifeways Cross- } \\
\text { Generation }\end{array}$} & \multirow{2}{*}{$\begin{array}{l}\text { Healthy Eating Index (HEI-2015), higher } \\
\text { score reflecting greater adherence to } \\
\text { DGA } \\
\text { assessed once during first trimester of } \\
\text { pregnancy }\end{array}$} & HEI 2015 & Age, SES, Smoking, Family history, HMF practices \\
\hline & & $\begin{array}{l}\text { - Over 10y follow-up ( } n=862) \text {, per } \\
\text { SD score increase of HEl-2015 } \\
\text { o aOR=0.78, 95\% Cl=(0.64, 0.94), } \\
P<0.05\end{array}$ & OFCs accounted for: \\
\hline
\end{tabular}




\section{Study and Participant}

Characteristics

Baseline $\mathrm{N}=862$ Analytic

$\mathrm{N}=862$

- Age: $30.4 \pm 5.8 y$

- Race/Ethnicity: 100\% Irish-

born

- SES:

-50.9\% $\geq$ tertiary education

o $15.8 \%$ eligible for free

healthcare

- Baseline characteristics

that differed by exposure:

o Age

o SES

o Smoking

\section{Intervention/Exposure and Outcomes Results}

\section{Dietary assessment:}

Mothers in the cohort completed a selfadministered semi quantitative 149 -item FFQ. The mothers indicated their average consumption frequency for each food item, ranging from "never or less than once per month" to " $6+$ per day," during the first $12-16$ wk of pregnancy.

The HEl-2015 scores a diet based on 9 adequacy components (intakes of total fruits; whole fruits; vegetables; greens and beans; total protein-containing foods; seafood and plant protein; whole grains; dairy; and ratio of PUFAs and MUFAs to SFAs) and 4 moderation components (refined grains, sodium, added sugars, and SFAs). The theoretical range of the HEI-2015 is 0100 , with a higher score reflecting greater adherence to recommendations in the dietary guideline for Americans (high intakes of foods counting toward adequacy components and low intakes of foods counting toward moderation components) and thus indicative of better dietary quality. For the adequacy components, a maximum score of 5 , reflecting a higher intake, can be awarded for intakes of total fruits, whole fruits, vegetables, greens and beans, total protein-containing foods, and seafood and plant protein; the maximum score is 10 for consumption of whole grains, dairy, and fatty acids

Confounding, Study Limitations, and Summary of Findings

Child Sex, Maternal Alcohol Use, Indoor/Outdoor

- At 3y $(n=677)$, per SD score increase

$\circ \mathrm{aOR}=0.70,95 \% \mathrm{Cl}=(0.52,0.93)$, $\mathrm{P}<0.05$

○ Sensitivity analyses excluding improbable energy intakes did not change results

- Over 10 y follow-up, per score quartile, $P$ for trend $=0.029$ ०Q2-Q4 vs Q1 (Ref): P=NS

- At 3 y, per score quartile, $P$ for trend $=0.016$ QQ2-Q3 vs Q1 (Ref): $P=N S$

○Q4 vs $Q 1: P<0.05$ (specific numbers only available for mutually adjusted model)

DII

- Over 10 y follow-up ( $n=862)$, per SD score increase of DII increase ○ $\mathrm{aOR}=1.34,95 \% \mathrm{Cl}=(1.09,1.64)$, $\mathrm{P}<0.01$

o Sensitivity analyses excluding improbable energy intakes did not change results

- At 3 y $(n=677)$, per SD score increase

o $\mathrm{aOR}=1.45,95 \% \mathrm{Cl}=(1.05,1.98)$ $\mathrm{P}<0.05$

- Sensitivity analyses excluding improbable energy intakes did not change results
Environment

\section{Limitations:}

- At least one key confounder not controlled for

- Adjusted for post-exposure variables

- Selection into the analysis was related to exposure and outcome

- Proportions of and reasons for missing participants may differ across exposure groups and the analysis is unlikely to have removed the risk of bias arising from the missing data

- No pre-registered data analysis plan

\section{Summary:}

A maternal diet during pregnancy that is of lower quality is associated with a higher risk of childhood asthma over the first $10 \mathrm{y}$.

A maternal diet during pregnancy that is of higher inflammatory potential is associated with a higher risk of childhood asthma over the first $10 \mathrm{y}$. 
Study and Participant

Characteristics

\section{Intervention/Exposure and Outcomes Results}

ratio. All moderation components were

subjected to reverse scoring and have a maximum score of 10 , reflecting a lower intake.

A higher E-DII score indicates a more proinflammatory diet.

\section{Outcomes:}

Doctor-diagnosed asthma in child over a 10-y follow-up (at ages 3, 5, and 9 y).

\section{Outcome assessment methods:}

At $3 \mathrm{y}$, doctors directly reported asthma diagnosis. At 5 y, information on doctordiagnosed asthma was obtained through parental report. At $9 \mathrm{y}$,

information on asthma diagnosis was obtained through both doctors' and parental reports.
- Sensitivity analysis excluding

based on $<500$ or $>3500 \mathrm{kcal} / \mathrm{d}$

o At 5 y $(n=543)$, per SD score

increase

o $\mathrm{aOR}=1.44,95 \% \mathrm{Cl}=(1.05,1.98)$,

$$
\mathrm{P}<0.05
$$

- At 9 y $(n=444)$, per SD score increase

o $\mathrm{aOR}=1.43,95 \% \mathrm{Cl}=(1.08,1.89)$,

$\mathrm{P}<0.05$

$\circ$ (Results similar for sensitivity analysis excluding based on $<500$ or $>5000 \mathrm{kcal} / \mathrm{d}$ )

- Over 10 y follow-up, per score quartile, $P$ for trend $=0.002$ $\circ Q 2$ vs $Q 1$ (Ref): $P=N S$ $\circ Q 3$ vs $Q 1$ (Ref): $P<0.05$ (specific numbers only available for mutually adjusted model) ○Q4 vs Q1 (Ref): aOR=2.28, 95\% $\mathrm{Cl}=(1.30,3.98)$

- At 3 y, per score quartile, $P$ for trend $=0.012$

○Q2-Q4 vs Q1 (Ref): P<0.05 (specific numbers only available for mutually adjusted model)

- At 9 y, per score quartile, $P$ for trend $=0.011$

$\circ Q 2$ vs $Q 1$ (Ref): $P=N S$

○Q3-Q4 vs Q1 (Ref): $P<0.05$ (specific numbers only available for mutually adjusted model) 
Study and Participant

Characteristics
Intervention/Exposure and Outcomes Results

Non-significant:

HEI-2015

- At 5 y ( $n=543)$, per SD score increase, $\mathrm{P}=\mathrm{NS}$

- At 9 y $(n=444)$, per SD score

increase, $\mathrm{P}=\mathrm{NS}$

- Sensitivity analyses excluding improbable energy intakes did not change results

- At 5 y, per score quartile, $P$ for trend $=0.42$ ०Q2-Q4 vs Q1 (Ref): P=NS

- At 9 y, per score quartile, $P$ for trend $=0.50$

०Q2-Q4 vs Q1 (Ref): P=NS

DII:

- At 5 y, per SD score increase, $\mathrm{P}=\mathrm{NS}$

- Sensitivity analyses excluding based on $<500$ or $>5000 \mathrm{kcal} / \mathrm{d}$ did not change results

- At 9 y, per SD score increase $\mathrm{P}=\mathrm{NS}$

Association between E-DII score quartiles and doctor-diagnosed asthma:

- At $5 \mathrm{y}$, per score quartile, $P$ for trend $=0.077$

QQ2-Q4 vs Q1 (Ref): P=NS
Confounding, Study Limitations, and Summary

of Findings 


\section{Study and Participant}

Characteristics

\section{Nguyen, 201727; The \\ Netherlands}

\section{PCS, Generation R}

Baseline $\mathrm{N}=5225$ Analytic $\mathrm{N}=3600$

- Age: Median=31.7y, IQR=28.4-34.4y

- Race/Ethnicity: NR for mother

- SES:

- Higher educational level: $52.7 \%$

o Household income $\geq 2200$ Euros/mo: $61.2 \%$

- Other characteristics reported but no test for differences by exposure o Smoking

- Family history of eczema, asthma

o HMF

- Animals/Pets/Farming exposure

\section{Intervention/Exposure and Outcomes Results}

\section{Food(s) or Food Group(s):}

Diet Quality Score

Higher diet quality included:

- High intake of vegetables, fruit, whole grains, legumes, nuts, dairy, fish, and tea

- High ratio whole grains to total grains and soft fats (i.e. soft margarines) and oils to total fat

- Low intake of red meat, sugarcontaining beverages, alcohol, and salt

- Folic acid supplement use in early pregnancy

\section{Dietary assessment methods:}

Validated FFQ in early pregnancy (median 13.6 wks gestation (IQR=12.416.2 wks). FFQ included foods

frequently consumed in the Dutch population and was modified for use in pregnant women. Previously developed predefined food-based diet quality score was applied, reflecting adherence to Dutch dietary guidelines. Diet quality score included continuous scores on 15 components: vegetables, fruit, whole grains, legumes, nuts, dairy, fish, tea, ratio whole grains of total grains, ratio soft fats (i.e., soft margarines) and oils of total fat, red meat, sugar-containing beverages, alcohol, salt, and folic acid supplement use in early pregnancy. Maximum score for each component was 1 , with an overall score ranging

Confounding, Study Limitations, and Summary of Findings

\section{Significant:}

\section{Non-significant:}

Risk of ever asthma at 10 y per 1 point higher diet quality score $(\mathrm{n}$ $=3,610), \mathrm{P}=\mathrm{NS}$

Results of a sensitivity analysis were consistent with the main findings

\section{Limitations:}

- Limited generalizability

- At least one key confounder not controlled for

- Methods used to assess the exposure likely to result in minimal exposure misclassification

- Proportions of and reasons for missing participants may differ across exposure groups and the analysis is unlikely to have removed the risk of bias arising from the missing data

- Outcomes were subjective and assessed by participants, who were aware of the exposure received

- No pre-registered data analysis plan

\section{Summary:}

Diet quality during pregnancy was not associated with risk of asthma in childhood. 
from 0 to 15. A higher score

represented better diet quality.

Outcome: Eczema, asthma at $10 \mathrm{y}$

Outcome assessment methods:

Positive response to question "Was your child ever diagnosed by a

physician with eczema/asthma?"

\section{Bertelsen, $2014^{3}$; Norway PCS, MoBa}

Baseline N=40614 Analytic $\mathrm{N}=40614$

- Age:

$0<20-24 y: 9 \%$

$\circ \geq 35 \mathrm{y}: 18 \%$

- Race/Ethnicity: NR

- SES:

o Single: $3 \%$

o Maternal Education:

- <High school: $6 \%$

- >4y college: $23 \%$

- Reported but not tested by exposure: Smoking, Family history, BW, GA, Delivery mode, $\mathrm{HMF}$

\section{Food(s) or Food Group(s):}

- Unexposed: No consumption of probiotic milk and yogurt, $\mathrm{n}=25,572$ (63\%)

- Exposed: Maternal consumption of probiotic milk and yogurt $n=15,042$ (37\%)

during pregnancy (at $22 \mathrm{wk}$ )

\section{Dietary assessment methods:}

Intake of milk-based probiotic products during pregnancy was recorded in the FFQ. The women were asked how often they consumed milk and yogurt, clearly distinguishing probiotic milk and yogurt from other milk items. Reported pregnancy

consumption across all probiotic milk products was categorized into one dichotomous variable for any intake versus no intake and one 3-level variable based on intake in milliliters per

\section{Significant:}

\section{Non-Significant:}

Asthma at $36 \mathrm{mo}, \mathrm{P}=\mathrm{NS}$

- Sensitivity analyses, $P=N S$

- Maternal intake ONLY

o Maternal AND child intake

- Maternal history of allergy/asthma

(Yes, No)

- Delivery mode

oSex

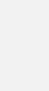

\section{Key confounders accounted for: \\ Age, SES, Smoking, Family history, HMF, Delivery mode}

\section{OFCs accounted for:}

Child sex

\section{Limitations:}

- Based on the exposure data availability, only a sub-set of women from MoBa were included in this study. Mothers who participated in this study were different than the rest, on the following

characteristics: education, smoking and parity

- Outcome measurement was subjective and were assessed by participants, who were aware of the exposure received

\section{Summary:}

Maternal consumption of probiotic milk and yogurt during pregnancy was not associated with risk of current asthma with asthma medication in the child at $36 \mathrm{mo}$. 


\begin{tabular}{|c|c|c|c|}
\hline $\begin{array}{l}\text { Study and Participant } \\
\text { Characteristics }\end{array}$ & Intervention/Exposure and Outcomes & Results & $\begin{array}{l}\text { Confounding, Study Limitations, and Summary } \\
\text { of Findings }\end{array}$ \\
\hline & $\begin{array}{l}\text { day categorized as "none," "13.0-28.3 } \\
\mathrm{mL} / \mathrm{d}, " \text { and ">28.4 mL/d" } \\
\text { Outcome: } \\
\text { Current asthma at } 36 \mathrm{mo} \text { with asthma } \\
\text { medication }\end{array}$ & & \\
\hline $\begin{array}{l}\text { Willers, } 2007^{31} \text {; U.K. } \\
\text { PCS } \\
\text { Baseline N=1751 Analytic } \\
\mathrm{N}=1212 \\
\text { Of } 1751 \text { women who } \\
\text { completed FFQ during } \\
\text { pregnancy, } 1212 \text { completed } \\
\text { follow-up when children } \\
\text { were } 5 y \text { y. } \\
\text { - Age at recruitment: } 29.9 y, \\
\text { 95\% Cl: (29.6, 30.2) } \\
\text { - Race/Ethnicity: NR } \\
\text { - SES: } \\
\text { ○ Partner from non-manual } \\
\text { social class: } 62.7 \% \\
\text { ○ Age left full time } \\
\text { education: Median=18.5y, } \\
\text { IQR=(16.0, } 21.0) \\
\text { - Characteristics reported } \\
\text { but no test for differences } \\
\text { by exposure } \\
\text { O Smoking } \\
\text { O Family history } \\
\text { O BW } \\
\text { O HMF (Ever) }\end{array}$ & $\begin{array}{l}\text { Food(s) or Food Group(s): } \\
\text { Apple consumption by tertile } \\
\text { - T1: } 0-1 / w k, n=398 \\
\text { - T2: } 1-4 / w k, n=427 \\
\text { - T3: }>4 / \text { wk, } n=384 \\
\text { at } \sim 32 \text { wk gestation } \\
\text { Fish consumption } \\
\text { - Never, } n=107 \\
\bullet<1 / w k, n=255 \\
\bullet \geq 1 / w k, n=831 \\
\text { at } \sim 32 \text { wk gestation } \\
\text { Oily fish consumption } \\
\text { - Never, } n=629 \\
\bullet<1 / w k, n=414 \\
\bullet \geq 1 / w k, n=161 \\
\text { at } \sim 32 \text { wk gestation } \\
\text { Dietary assessment methods: } \\
\text { Semi-quantitative, } 150 \text {-item FFQ, } \\
\text { divided into } 20 \text { food groups to capture } \\
\text { intake over the previous } 2-3 m o \text {. } \\
\text { Responses were categorized as rarely } \\
\text { or never, } 1-2 \text { times/mo, and separate } \\
\text { categories for } 1-7 \text { d/wk. Food amounts }\end{array}$ & $\begin{array}{l}\text { Non-significant: } \\
\text { Asthma and wheeze in last } 12 \text { mo } \\
\text { - Apple Intake, Cases } \mathrm{n}=145, \mathrm{P} \text { for } \\
\text { trend=NS } \\
\text { There were no consistent linear } \\
\text { associations between maternal } \\
\text { intake of total fruit, citrus/kiwi fruit, } \\
\text { total vegetables, green leafy } \\
\text { vegetables, fruit juice, whole grain } \\
\text { products, fat from dairy products or } \\
\text { butter versus margarine/low fat } \\
\text { spread use and respiratory or atopic } \\
\text { outcomes in children at } 5 \mathrm{y} \text {, nor were } \\
\text { there consistent associations } \\
\text { between maternal intake of food }\end{array}$ & $\begin{array}{l}\text { Key confounders accounted for: } \\
\text { Age, SES, Smoking (Mother, Household), Family } \\
\text { history, BW, HMF } \\
\text { OFCs accounted for: } \\
\text { Child sex } \\
\text { Limitations: } \\
\text { - At least one key confounder not controlled for } \\
\text { - Mothers who participated in the study were of } \\
\text { higher SES and had slightly higher consumption of } \\
\text { fruits, green leafy vegetables, whole grain } \\
\text { products and fish. They also had fewer respiratory } \\
\text { symptoms. } \\
\text { - Multiple exposure outcome comparisons were } \\
\text { assessed without using an appropriate p-value } \\
\text { correction } \\
\text { - No pre-registered data analysis plan } \\
\text { Summary: } \\
\text { Higher maternal apple intake was associated with } \\
\text { lower odds of doctor-confirmed asthma in the child } \\
\text { at } 5 y, \text { but not significantly associated with asthma } \\
\text { and wheeze in the previous } 12 m o \text {. }\end{array}$ \\
\hline
\end{tabular}


obtain the total measures/wk.

The food groups of interest in this study were total fruit, citrus/kiwi fruit, apples,

total vegetables, green leafy

vegetables, pure fruit juice, whole grain products, total fish, total oily fish, total fat from dairy products and exclusive butter versus margarine/low fat spread used as spread. The total number of measures per week was divided into tertiles for total fruit, citrus/kiwi fruit, apples, total vegetables, green leafy vegetables, pure fruit juice, whole grain products and into the categories never, less than once a week, and once or more a week for total fish and total oily fish. To facilitate extrapolation to the general population, subdivisions of food intakes into tertiles were derived from all of the women completing the FFQ and not merely those responding at 5 years.

\section{Outcome: Asthma}

\section{Outcome assessment methods:}

Based on ISAAC core questions on symptoms of asthma, including the questions: "Has your child ever suffered from asthma?", "Has this been confirmed by a doctor?" and "Has your child received treatment for asthma in the past 12 months?' 
Study and Participant

Characteristics
Results
Confounding, Study Limitations, and Summary

of Findings
Willers, 2008 ${ }^{32}$; The

Netherlands

\section{PCS, PIAMA}

Baseline $\mathrm{N}=3963$

Analytic $\mathrm{N}=2832$

- Age at Delivery: $30.3 \pm$ 3.9y

- Race/Ethnicity: NR

- SES:

- Low maternal educational level: $22.6 \%$

- Low partner's educational level: $24.6 \%$

Baseline characteristics NR by exposure

\section{Food(s) or Food Group(s):}

Consumption frequency (rarely, regularly, daily) of following food groups: vegetables, fruit, fish, egg, dairy, nuts, and nut products

$\sim 80 \%$ completed questionnaire between 30th and 36th wk of gestation

\section{Outcome:}

Asthma at 3-8y

\section{Outcome assessment methods:}

Self-reported prescription of inhaled corticosteroids for respiratory problems in the last $12 \mathrm{mo}$

Self-reported doctor-diagnosed asthma in the last $12 \mathrm{mo}$

\section{Significant:}

Doctor-Diagnosed Asthma From 38y:

- Nut Product Consumption

○ Daily vs Rarely (Ref): 1.64, 95\%

$\mathrm{Cl}=(1.03,2.60), \mathrm{P}<0.05$

Steroid Use From 3-8y:

- Nut Product Consumption

$\circ$ Daily vs Rarely (Ref): $\mathrm{aOR}=1.62$, $95 \% \mathrm{Cl}=(1.06,2.46), \mathrm{P}<0.05$

Steroid Use at 4y:

- Nut Product Consumption

o Daily vs Rarely (Ref): $P<0.05$

Steroid Use at 6y:

- Fruit Consumption

$\circ$ Daily vs Regularly + Rarely (Ref): $\mathrm{P}<0.05$

- Nut Product Consumption

o Daily vs Rarely (Ref): $\mathrm{P}<0.05$

\section{Non-Significant:}

Doctor-Diagnosed Asthma From 38y:

- Vegetables, Fruit, Dairy

Consumption

○Daily vs Regularly + Rarely (Ref):

$\mathrm{P}=\mathrm{NS}$

- Fish, Egg, Nuts Consumption

\section{Key confounders accounted for:}

SES, Smoking, Family history, BW, HMF practices

\section{OFCs accounted for:}

Child Sex

\section{Limitations:}

- At least one key confounder not controlled for

- Selection into the study was related to exposure and outcome and this could not be adjusted for in analyses

- No information on deviation from intended exposures

- No pre-registered data analysis plan

\section{Summary:}

High maternal nut product intake during pregnancy is associated with a higher risk of childhood asthma at 3-8 years, compared with low intake.

Higher maternal fruit product intake during pregnancy is associated with a reduced risk of childhood asthma at $6 y$, compared with low intake.

Maternal vegetable, dairy, fish, egg, and nut intake during pregnancy was not associated with risk of childhood asthma at 3-8 y. 
Study and Participant

Characteristics
Intervention/Exposure and Outcomes Results

oDaily + Regularly vs Rarely (Ref):

$\mathrm{P}=\mathrm{NS}$

- Nut Product Consumption

o Regularly vs Rarely (Ref): $\mathrm{P}=\mathrm{NS}$

Steroid Use From 3-8y:

- Vegetables, Fruit, Dairy

Consumption

- Daily vs

$$
\begin{aligned}
& \text { Regularly + } \\
& \text { Rarely (Ref): } \\
& \mathrm{P}=\mathrm{NS}
\end{aligned}
$$

- Fish, Egg, Nuts Consumption

$$
\begin{aligned}
& \text { Daily }+ \\
& \text { Regularly vs } \\
& \text { Rarely (Ref): } \\
& \mathrm{P}=\mathrm{NS}
\end{aligned}
$$

- Nut Product Consumption

o Regularly vs Rarely (Ref): $\mathrm{P}=\mathrm{NS}$

Steroid Use at 3, 4, 5, 7, and 8y:

- Fruit Consumption

$\circ$ Daily vs Regularly + Rarely (Ref):

$$
\mathrm{P}=\mathrm{NS}
$$

Steroid Use at 3, 5, 7, and 8y:

- Nut Product Consumption

$\circ$ Daily vs Rarely (Ref): $\mathrm{P}=\mathrm{NS}$

$$
\circ \text { Daily vs Rarely (Ref): } \mathrm{P}=\mathrm{NS}
$$

Confounding, Study Limitations, and Summary of Findings

Pregnancy and Lactation

Randomized Controlled Trials

Fukushima, $1997^{8}$; Japan Food(s) or Food Group(s):
Baseline $\mathrm{N}=350$ Analytic

$\mathrm{N}=283$

\section{Significant:}

Note, CD had significantly higher odds of overall allergies vs MD at

\section{Key confounders accounted for:}

Smoking, Family history, (CFB for aOR), Pets 


\section{Study and Participant}

Characteristics

- Age: NR

- Race/Ethnicity: 100\% from

Japan

- SES: NR

- Significant differences by exposure (by design)

HMF, CFB

- No differences by exposure: Smoking, Family history, BW, Pets

$\sim 76 \%$ had a positive history of allergy (i.e. both parents or one parents or sibling only had history of allergies)

\section{Intervention/Exposure and Outcomes Results}

- CD: Mothers instructed to consume

$>200 \mathrm{~mL} / \mathrm{d}$ cow milk, $\mathrm{n}=140$

randomized, $\mathrm{n}=127$ analyzed

- MD: Mothers instructed to consume $>200 \mathrm{~mL} / \mathrm{d}$ a casein-free,

hypoallergenic formula and to avoid cow milk and reduce consumption of cow milk products, $\mathrm{n}=140$ randomized, $\mathrm{n}=102$ analyzed

$12 \mathrm{mo}$, but due to inclusion of asthma

these results to not meet NESR

criteria

\section{Non-significant:}

Asthma prevalence at $24 \mathrm{mo}, \mathrm{P}=\mathrm{NS}$ - CD: $a O R=0.79,95 \% \mathrm{Cl}:(0.24$, 2.54)

-MD: (Ref)

$>200 \mathrm{~mL} / \mathrm{d}$ a casein-free,

hypoallergenic formula and to avoid cow milk and reduce consumption of cow milk products, $\mathrm{n}=70$ randomized, $\mathrm{n}=54$ analyzed (Not randomized, no relevant comparisons included, except as co-variates in logistic regressions) - Also supplemented with $1000 \mathrm{mg} / \mathrm{d}$ $\mathrm{Ca}$

during late pregnancy through end of lactation up to 6 mo postpartum

From birth to $6 \mathrm{mo}$, the infants in the $M D$ and $C D$ groups were exclusively HMF or mixed-fed with human milk and casein-free, hypoallergenic formula when human milk was insufficient. The infants in the AF group were mixed-fed with human milk and a formula with similar whey:casein ratio as human milk for the corresponding $6 \mathrm{mo}$. Infants who were fed human milk exclusively from birth to $4 \mathrm{mo}$ were excluded from the AF group.

Overall allergies at $24 \mathrm{mo}, \mathrm{P}=\mathrm{NS}$ - CD: aOR=1.75, 95\% Cl: $(0.94$, 3.25)

-MD: (Ref)

Note, odds of overall allergies NS at 6 and $18 \mathrm{mo}$. However, due to inclusion of asthma these results do not meet the NESR criteria
Confounding, Study Limitations, and Summary of Findings

\section{OFCs accounted for:}

None

\section{Limitations:}

- Randomization process NR

- MD still consumed cow milk products, but had lower intake than $C D$

- Attrition higher in MD group because women excluded from analysis if they consumed cow milk

Proportions of missing data seem to differ. reasons for missingness NR

- Participants were aware of exposure status and outcomes were self-reported

- Eczema logistic regressions only reported at 12 and $24 \mathrm{mo}$, but data were collected at all 4 time points

- Pre-registered data analysis plan NR

\section{Summary:}

Consuming diets replacing cow milk with casein-free hypoallergenic formula with reduced consumption of cow milk products from late pregnancy through lactation did not impact overall allergies, including atopic eczema, asthma, and allergic rhinitis.

\section{Dietary assessment methods}




\section{Outcomes:}

Overall allergies (eczema, asthma, and/or allergic rhinitis)

at $24 \mathrm{mo}$

\section{Outcome assessment methods:}

Self-reported by questionnaire. Specific allergies defined when symptoms chronically lasted for more than a few weeks.

Asthma was considered when cough and other respiratory symptoms were observed without any suspicion of infections.

\section{Zeiger, $1992^{36}$; U.S.}

RCT

Baseline $\mathrm{N}=379$ Analytic $\mathrm{N}=242$ (at $3 \mathrm{y}$ ) and 225 (at $4 y)$

- Age: $28.6 y$

- Race/Ethnicity: o Non-White: $~ 12.5 \%$

- SES:

\section{Food(s) or Food Group(s):}

- Control: Mothers encouraged to follow standard diets during 3rd trimester of pregnancy and lactation

- Prophylaxis: Avoided all milk (dairy), egg, and peanut products,

concentrated soy foods (i.e., tofu), $\leq 2$ servings/d wheat during 3rd trimester of pregnancy and lactation

- Prenatal vitamins plus supplemented with a total of $1500 \mathrm{mg} / \mathrm{d} \mathrm{Ca}$

Both groups encouraged to feed human milk for $\geq 4-6$ mo

\section{Significant:}

\section{Non-Significant:}

The period prevalence of asthma was similar in the prophylactictreated and control groups at 2, 3, and $4 \mathrm{y}$.

\section{PES:}

\section{Key confounders accounted for: \\ None}

\section{OFCs accounted for:}

None

Limitations: (Note: most of these limitations were identified based on Zeiger 1989 paper)

- More women in the prophylactic-treated group withdrew before delivery because of the protocol's dietary restrictions. As a result, halfway through recruitment, randomization was changed from 


\section{Study and Participant}

Characteristics

o Maternal occupation:

white collar: $~ 54.0 \%$

- Family income

$<\$ 20,000 / \mathrm{y}: ~ 9.7 \%$

- Maternal Education:

SHigh school: $\sim 13.6 \%$

- Significant differences by exposure: BW (term, singletons)

- No differences by exposure: Smoking, Family history, HMF, CFB, Animal/Pets/Farming exposure

Families were included in the study if at least one parent met the following criteria: history of an atopic disorder and specific $\lg \mathrm{E}$ by skin or RAST testing. Serum was obtained from the participants for total and specific lgE determinations. Participant fathers were skin tested to inhalant antigens at the intake session. Mothers were skin tested to foods and inhalants $4 \mathrm{mo}$ postpartum.

\section{Intervention/Exposure and Outcomes Results}

\section{Outcome:}

Allergic Rhinitis at $3 y$ and $4 y$

Period prevalence: Defined as the proportion of participant currently evidencing a measured parameter

Cumulative prevalence: Defined as the proportion of participants evidencing the measured parameter at any past or current time

Prophylactic infants used casein hydrolysate (Nutramigen) for supplementation or weaning, and avoided solid foods for $6 \mathrm{mo}$; cow milk, corn, soy, citrus, and wheat, for $12 \mathrm{mo}$; and egg, peanut, and fish, for $24 \mathrm{mo}$. Infants in the control groups Enfamil, a cow milk-based whey infant formula.

Solid foods were introduced to controlgroup infants, based on AAP recommendations.
Confounding, Study Limitations, and Summary of Findings

40:60 (treatment: control) to 50:50 with a new computerized list of random numbers

- Rate of drop-out at baseline was significantly different in the prophylaxis vs. control groups $(\mathrm{p}<0.0001)$

- Significantly more non-White fathers in prophylaxis group, and trend to more mothers with low education in control group

- 6 pairs of twins omitted from control group after randomization

- BW $2.9 \%$ lower in term, singletons in prophylactic group ( $\mathrm{P}=0.044)$; Overall, BW similar between groups

- Smoking during postpartum was significantly different between the prophylactic and control groups

\section{Summary:}

Maternal avoidance of cow milk, egg, and peanut during the last trimester of pregnancy and lactation, as well as infant avoidance of cow milk until 1 year and egg, peanut, and fish until after 2 y did not result in a lower risk of asthma at 2, 3, and 4 years compared to a control diet.

\section{Zeiger, $1995^{34}$; U.S. \\ RCT \\ Baseline N=379 Analytic $\mathrm{N}=165$}

\section{Food(s) or Food Group(s)}

- Control: Mothers encouraged to follow standard diets during $3^{\text {rd }}$ trimester of pregnancy and lactation, $\mathrm{n}=212$

o Cow milk-based whey infant formula provided for

\section{Significant:}

Non-Significant:

Period prevalence, $P=N S$
Key confounders accounted for:

None

OFCs accounted for: 


\section{Study and Participant}

Characteristics

Power analysis: Yes

Of these families, 14 in each group were found to be not atopic, which eliminated them from the study

\section{See Zeiger, $1992^{36}$}

\section{Intervention/Exposure and Outcomes Results}

supplementation or weaning through $12 \mathrm{mo}$ postpartum. CFB encouraged: no solids $<4 \mathrm{mo}$, cereal at $4 \mathrm{mo}$, followed by vegetables, fruits and egg yolks at

$6 \mathrm{mo}$, meats at $8 \mathrm{mo}$, and whole cow milk and egg whites at $12 \mathrm{mo}$.

- Prophylaxis: Instructed to avoid totally all milk (dairy), egg, and peanut products, avoid concentrated soy foods (i.e., tofu), $\leq 2$ servings/d wheat, with other grains to fulfill cereal and starch requirements during $3^{\text {rd }}$ trimester of pregnancy and lactation, $\mathrm{n}=167$

- In addition to prenatal vitamins, the maternal diet was supplemented with a total of $1500 \mathrm{mg} / \mathrm{d} \mathrm{Ca}$.

- A casein hydrolysate infant formula with low sensitization potential provided for supplementation or weaning through $12 \mathrm{mo}$ postpartum. CFB encouraged: no solids $<6 \mathrm{mo}$, non-legume vegetables, followed by rice cereal at $7 \mathrm{mo}$, meats at $8 \mathrm{mo}$, non-citrus fruits and juices at $9 \mathrm{mo}$, and cow milk at $12 \mathrm{mo}$. Wheat, soy, corn, and citrus introduced thereafter at monthly intervals, followed by egg at $24 \mathrm{mo}$ and peanuts and fish at $36 \mathrm{mo}$.

- Both groups encouraged to feed human milk for $\geq 4-6 \mathrm{mo}$

\section{Dietary assessment methods:}

Women were randomly assigned to groups. In addition to instructions
Confounding, Study Limitations, and Summary of Findings

None

Cumulative prevalence, $\mathrm{P}=\mathrm{NS}$

Limitations: (Note: most of these limitations were identified based on Zeiger 1989 paper)

- More women in the prophylactic-treated group withdrew before delivery because of the protocol's dietary restrictions. As a result, halfway through recruitment, randomization was changed from 40:60 (treatment: control) to 50:50 with a new computerized list of random numbers

- Rate of drop-out at baseline was significantly different in the prophylaxis vs. control groups $(p<0.0001)$

- Significantly more non-White fathers in prophylaxis group, and trend to more mothers with low education in control group

- 6 pairs of twins omitted from control group after randomization

- BW $2.9 \%$ lower in term, singletons in prophylactic group $(\mathrm{P}=0.044)$; Overall, BW similar between groups

- Smoking during postpartum was significantly different between the prophylactic and control groups

\section{Summary:}

Maternal avoidance of milk (dairy), egg, and peanut products, avoid concentrated soy foods, and limited intake of wheat during the 3rd trimester of pregnancy through lactation and reduced infant food allergen exposure during CFB did not affect the prevalence of asthma in the child at $7 \mathrm{y}$. 
Study and Participant

Characteristics
Intervention/Exposure and Outcomes

Results

described above, women in prophylaxis

group attended a dietary class was held before the 3rd trimester by a licensed

dietitian to provide detailed instructions on the maternal and infant diets, food

lists, recipes, and product sources.

Adherence to the dietary regimen was ascertained in part by maternal self-

report and daily diaries.

For both groups: $0.25 \mathrm{mg} / \mathrm{d}$ Tri-Vi-Flor given to infants according to their pediatrician's preference. Foods causing documented IgE sensitization were removed from the infant's diet until sensitization had waned or were tolerated on double-blind challenge.

Parents received intensive education on reducing environmental allergens and tobacco smoke from their homes.

Outcome: Atopic dermatitis, Allergic rhinitis, Food allergy

at $1,4,8$, and $12 \mathrm{mo}$

\section{Outcome assessment methods:}

Allergic rhinitis was defined as a nasal condition with characteristic symptoms of sneezing, itching, and/or rhinorrhea with

concurrent specific lgE and nasal eosinophils (NEs)
Confounding, Study Limitations, and Summary of Findings

\section{Lactation}

\section{Prospective Cohort Studies}


Study and Participant

Characteristics

\section{Lumia, 2012 38 ; Finland} PCS, DIPP

Baseline $\mathrm{N}=1798$

Analytic $\mathrm{N}=1798$

- Age at Delivery: 30.1 $4.9 y$

- Race/Ethnicity: NR

- SES: NR

Baseline characteristics NR by exposure

\section{Intervention/Exposure and Outcomes Results}

\section{Food(s) or Food Group(s):}

Intake $(\mathrm{g} / \mathrm{d})$ categorized into quartiles for following food groups: oils, butter and butter-spreads, industrial fat, fish, meat and meat products, and milk and milk products

- Margarine intake ( $\mathrm{g} / \mathrm{d})$ dichotomized into user/nonuser

Assessed once during third month of lactation

\section{Dietary assessment:}

Dietary intake during lactation was assessed by a validated, selfadministered, semi-quantitative 181item FFQ. The FFQ was designed to assess the maternal total diet (intake of foods and nutrients) during lactation, over a period of 1 month. The mothers were asked to circle the right picture

of the amount of margarine on one slice of bread they used. The consumption frequency was also asked. The use of margarines was classified depending on their fat content: margarines containing $70-80 \%$ or $30-60 \%$ fat, and brand names were given.

\section{Outcome:}

Current asthma at 5y

\section{Outcome assessment methods:}

Self-reported doctor-diagnosis plus any wheezing symptom or the use of

\section{Significant:}

Margarines

- User vs nonuser: aHR=1.96, 95\% $\mathrm{Cl}=(1.01,3.82), \mathrm{P}=0.047$

\section{Non-Significant:}

Oils

- Q1 and Q4 vs Q2/Q3 combined (Ref): $P=N S$

Butter and butter-spreads

- Q1 and Q4 vs Q2/Q3 combined (Ref): $P=N S$

Industrial Fat

- Q1 and Q4 vs Q2/Q3 combined (Ref): $P=N S$

Fish

- Q1 and Q4 vs Q2/Q3 combined (Ref): $P=N S$

Meat and meat products

- Q1 and Q4 vs Q2/Q3 combined (Ref): $P=N S$

Milk and milk products

- Q1 and Q4 vs Q2/Q3 combined (Ref): $P=N S$
Confounding, Study Limitations, and Summary of Findings

\section{Key Confounders accounted for:}

Age, SES, Smoking, Family history, GA, BW, Delivery mode, HMF practices,

Animals/pets/farming exposure

\section{OFCs accounted for:}

Child Sex

\section{Limitations:}

- At least one key confounder not controlled for

- Adjusted for post-exposure variable

- Outcome may have been subjective and was assessed by participants, who were aware of exposure received

- No information on missing outcome data by exposure group

- No pre-registered data analysis plan

\section{Summary:}

Maternal margarine intake during lactation is associated with a higher risk of childhood asthma at $5 y$, compared to no intake.

Maternal oil, butter and butter-spread, industrial fat, fish, meat and meat product, and milk and milk product intake during lactation are not associated with risk of childhood asthma at $5 y$. 
Study and Participant

Characteristics

Intervention/Exposure and Outcomes

Results

asthma medication during the previous

$12 \mathrm{mo}$
Confounding, Study Limitations, and Summary

of Findings 
Table 6. Risk of bias for randomized controlled trials examining maternal diet during pregnancy and lactation and risk of child food allergies and atopic allergic diseases ${ }^{\text {xiii, }}$ xix

\begin{tabular}{|l|c|c|c|c|c|}
\hline & Randomization & $\begin{array}{c}\text { Deviations from } \\
\text { intended } \\
\text { interventions }\end{array}$ & $\begin{array}{c}\text { Missing } \\
\text { outcome data }\end{array}$ & $\begin{array}{c}\text { Outcome } \\
\text { measurement }\end{array}$ & $\begin{array}{c}\text { Selection of the } \\
\text { reported result }\end{array}$ \\
\hline Falth-Magnusson, $\mathbf{1 9 8 7}^{7}$ & Low & High & Low & Low & Some concerns \\
\hline Fukushima, $\mathbf{1 9 9 7}^{8}$ & Some concerns & High & High & High & High \\
\hline Jirapinyo, $\mathbf{2 0 1 3}^{37}$ & Some concerns & Some concerns & Low & Low & Some concerns \\
\hline Lilja, $\mathbf{1 9 8 9} \mathbf{1 4}^{14}$ Lovegrove, $\mathbf{1 9 9 4}^{16}$ & Some concerns & Some concerns & Low & Low & High \\
\hline
\end{tabular}

Table 7. Risk of bias for non-randomized controlled trials examining maternal diet during pregnancy and lactation and risk of child food allergies and atopic allergic diseases ${ }^{\mathrm{xx}}$

\begin{tabular}{|c|c|c|c|c|c|c|c|}
\hline & Confounding & $\begin{array}{l}\text { Selection of } \\
\text { participants }\end{array}$ & $\begin{array}{l}\text { Classification } \\
\text { of exposures }\end{array}$ & $\begin{array}{l}\text { Deviations } \\
\text { from intended } \\
\text { exposures }\end{array}$ & Missing data & $\begin{array}{c}\text { Outcome } \\
\text { measurement }\end{array}$ & $\begin{array}{l}\text { Selection of } \\
\text { the reported } \\
\text { result }\end{array}$ \\
\hline Herrmann, $1996^{10}$ & Serious & Serious & Moderate & Serious & Moderate & Serious & Serious \\
\hline
\end{tabular}

xviii A detailed description of the methodology used for assessing risk of bias is available on the NESR website: https://nesr.usda.gov/2020-dietary-guidelinesadvisory-committee-systematic-reviews and in Part C of the following reference: Dietary Guidelines Advisory Committee. 2020. Scientific Report of the 2020 Dietary Guidelines Advisory Committee: Advisory Report to the Secretary of Agriculture and the Secretary of Health and Human Services. U.S. Department of Agriculture, Agricultural Research Service, Washington, DC.

xix Possible ratings of low, some concerns, or high determined using the "Cochrane Risk-of-bias 2.0" (RoB 2.0) (August 2016 version)" (Higgins JPT, Sterne JAC, Savović J, Page MJ, Hróbjartsson A, Boutron I, Reeves B, Eldridge S. A revised tool for assessing risk of bias in randomized trials In: Chandler J, McKenzie J, Boutron I, Welch V (editors). Cochrane Methods. Cochrane Database of Systematic Reviews 2016, Issue 10 (Suppl 1). dx.doi.org/10.1002/14651858.CD201601.)

${ }^{x x}$ Possible ratings of low, moderate, serious, critical, or no information determined using the "Risk of Bias in Non-randomized Studies of Interventions (ROBINS-I) tool" (Sterne JAC, Hernán MA, Reeves BC, Savović J, Berkman ND, Viswanathan M, Henry D, Altman DG, Ansari MT, Boutron I, Carpenter JR, Chan AW, Churchill R, Deeks JJ, Hróbjartsson A, Kirkham J, Jüni P, Loke YK, Pigott TD, Ramsay CR, Regidor D, Rothstein HR, Sandhu L, Santaguida PL, Schünemann HJ, Shea B, Shrier I, Tugwell P, Turner L, Valentine JC, Waddington H, Waters E, Wells GA, Whiting PF, Higgins JPT. ROBINS-I: a tool for assessing risk of bias in non-randomized studies of interventions. BMJ 2016; 355; i4919; doi: 10.1136/bmj.i4919.) 
Table 8. Risk of bias for observational studies examining maternal diet during pregnancy and lactation and risk of child food allergies and atopic allergic disease ${ }^{\mathrm{xxi}}$

\begin{tabular}{|c|c|c|c|c|c|c|c|}
\hline & Confounding & $\begin{array}{l}\text { Selection of } \\
\text { participants }\end{array}$ & $\begin{array}{l}\text { Classification } \\
\text { of exposures }\end{array}$ & $\begin{array}{l}\text { Deviations } \\
\text { from intended } \\
\text { exposures }\end{array}$ & Missing data & $\begin{array}{c}\text { Outcome } \\
\text { measurement }\end{array}$ & $\begin{array}{l}\text { Selection of } \\
\text { the reported } \\
\text { result }\end{array}$ \\
\hline Bédard, 2018² & Serious & Moderate & Moderate & Serious & Low & Moderate & Serious \\
\hline Bédard, $2020^{1}$ & Serious & Moderate & Moderate & No information & Low & Serious & Serious \\
\hline Bertelsen, 2014 ${ }^{3}$ & Serious & Moderate & Moderate & Moderate & Serious & Serious & Serious \\
\hline Bunyavanich, 2014 ${ }^{4}$ & Serious & Moderate & Moderate & No information & Serious & Moderate & Serious \\
\hline Chatzi, $2013^{5}$ & Serious & Serious & Serious & No information & No information & Serious & Serious \\
\hline Chen, $2020^{6}$ & Serious & Moderate & Moderate & Moderate & Serious & Moderate & Serious \\
\hline Hanson, $2020^{9}$ & Serious & Moderate & Moderate & Moderate & Low & Moderate & Serious \\
\hline Leermakers, $2013^{13}$ & Serious & Moderate & Moderate & Moderate & Moderate & Moderate & Serious \\
\hline Jedrychowski, $2011^{11}$ & Serious & Serious & Serious & Serious & No information & Serious & Serious \\
\hline Lack, $2003^{12}$ & Critical & Serious & Serious & No information & No information & Low & Critical \\
\hline Loo, $2017^{15}$ & Serious & Moderate & Moderate & No information & Moderate & Moderate & Serious \\
\hline Lumia, $2012^{38}$ & Serious & Moderate & Serious & Moderate & No information & Moderate & Serious \\
\hline Maslova, $\mathbf{2 0 1 2}^{17}$ & Serious & Serious & Moderate & Serious & Moderate & Moderate & Critical \\
\hline Maslova, $2012^{18}$ & Serious & Serious & Moderate & Serious & Moderate & Moderate & Critical \\
\hline Maslova, $\mathbf{2 0 1 3 ^ { 1 9 }}$ & Serious & Serious & Moderate & No information & No information & Serious & Serious \\
\hline Maslova, $2013^{20}$ & Serious & Serious & Moderate & Serious & Moderate & Moderate & Serious \\
\hline Miyake, $2009^{24}$ & Serious & Moderate & Moderate & No information & No information & Serious & Serious \\
\hline Miyake, $2010^{23}$ & Serious & Moderate & Moderate & No information & No information & Serious & Serious \\
\hline Miyake, $\mathbf{2 0 1 0 ^ { 2 2 }}$ & Serious & Moderate & Moderate & No information & No information & Serious & Serious \\
\hline Miyake, $\mathbf{2 0 1 1 ^ { 2 1 }}$ & Serious & Moderate & Moderate & No information & No information & Serious & Serious \\
\hline Miyake, $\mathbf{2 0 1 3}^{25}$ & Serious & Moderate & Moderate & No information & No information & Serious & Serious \\
\hline Miyake, $2014^{26}$ & Serious & Moderate & Moderate & No information & No information & Serious & Serious \\
\hline Nguyen, $2017^{27}$ & Serious & Moderate & Moderate & Moderate & No information & Moderate & Serious \\
\hline
\end{tabular}

xxi Possible ratings of low, moderate, serious, critical, or no information determined using the "Risk of Bias for Nutrition Observational Studies" tool (RoB-NObs) (Dietary Guidelines Advisory Committee. 2020. Scientific Report of the 2020 Dietary Guidelines Advisory Committee: Advisory Report to the Secretary of Agriculture and the Secretary of Health and Human Services. U.S. Department of Agriculture, Agricultural Research Service, Washington, DC.) 


\begin{tabular}{|l|l|l|l|l|c|c|c|}
\hline Saito, $\mathbf{2 0 1 0}^{28}$ & Serious & Moderate & Moderate & No information & No information & Serious & Serious \\
\hline Shaheen, $\mathbf{2 0 0 9}^{29}$ & Serious & Moderate & Moderate & Moderate & Serious & Serious & Serious \\
\hline Viljoen, $\mathbf{2 0 1 8}^{30}$ & Serious & Serious & Moderate & Moderate & Serious & Moderate & Critical \\
\hline Willers, $\mathbf{2 0 0 7}^{31}$ & Serious & Moderate & Moderate & Moderate & Serious & Moderate & Serious \\
\hline Willers, $\mathbf{2 0 0 8}^{32}$ & Serious & Serious & Moderate & No information & Serious & Moderate & Serious \\
\hline Wright, $\mathbf{2 0 1 8}^{33}$ & Serious & Moderate & Moderate & Moderate & Serious & Moderate & Serious \\
\hline
\end{tabular}




\section{METHODOLOGY}

The NESR team used its rigorous, protocol-driven methodology to support the 2020 Dietary Guidelines Advisory Committee in conducting this systematic review.

NESR's systematic review methodology involves:

- Developing a protocol,

- Searching for and selecting studies,

- Extracting data from and assessing the risk of bias of each included study,

- Synthesizing the evidence,

- Developing conclusion statements,

- Grading the evidence underlying the conclusion statements, and

- Recommending future research.

A detailed description of the methodology used in conducting this systematic review is available on the NESR website: https://nesr.usda.gov/2020-dietary-guidelines-advisory-committee-systematicreviews, and can be found in the 2020 Dietary Guidelines Advisory Committee Report, Part C: Methodology. ${ }^{x x i i}$ This systematic review was peer reviewed by Federal scientists, and information about the peer review process can also be found in the Committee's Report, Part C. Methodology. Additional information about this systematic review, including a description of and rationale for any modifications made to the protocol can be found in the 2020 Dietary Guidelines Advisory Committee Report, Chapter 2. Food, Beverage, and Nutrient Consumption During Pregnancy and Chapter 3. Food, Beverage, and Nutrient Consumption During Lactation.

Below are details of the final protocol for the systematic review described herein, including the:

- Analytic framework

- Literature search and screening plan

- Literature search and screening results

\section{ANALYTIC FRAMEWORK}

The analytic framework (Figure 1: Analytic frameworkFigure 1) illustrates the overall scope of the systematic review, including the population, the interventions and/or exposures, comparators, and outcomes of interest. It also includes definitions of key terms and identifies key confounders considered in the systematic review. The inclusion and exclusion criteria that follow provide additional information about how parts of the analytic framework were defined and operationalized for the review.

xxii Dietary Guidelines Advisory Committee. 2020. Scientific Report of the 2020 Dietary Guidelines Advisory Committee: Advisory Report to the Secretary of Agriculture and the Secretary of Health and Human Services. U.S. Department of Agriculture, Agricultural Research Service, Washington, DC. 


\section{Figure 1: Analytic framework}

\section{Systematic review question: What is the relationship between maternal diet during pregnancy and lactation and}

risk of child food allergies and atopic allergic diseases?

\begin{tabular}{|c|c|}
\hline Intervention/exposure vs Comparator & Outcomes \\
\hline $\begin{array}{l}\begin{array}{c}\text { Dietary intake of a food(s) } \\
\text { or food group(s) }\end{array} \\
\begin{array}{r}\text { No food or different } \\
\text { amount of dietary intake } \\
\text { of the same food(s) or } \\
\text { food group(s) }\end{array} \\
\begin{array}{l}\text { Population: Women during pregnancy and/or during lactation; } \\
\text { healthy and/or at risk for chronic disease }\end{array}\end{array}$ & $\begin{array}{l}\text { Food allergies } \\
\text { - Allergic rhinitis } \\
\text { Popic dermatitis } \\
\text { children and adolescents (ages 2-18 years) } \\
\text { - Asthma } \\
\text { Population: Children and adolescents (ages 2-18 } \\
\text { years) }\end{array}$ \\
\hline
\end{tabular}

\begin{tabular}{|l|l|}
\hline Legend \\
The relationship of interest in the \\
systematic review
\end{tabular}




\section{LITERATURE SEARCH AND SCREENING PLAN}

\section{Inclusion and exclusion criteria}

This table provides the inclusion and exclusion criteria for the systematic review. The inclusion and exclusion criteria are a set of characteristics used to determine which articles identified in the literature search were included in or excluded from the systematic review.

\section{Table 9. Inclusion and exclusion criteria}

\begin{tabular}{lll}
\hline Category & Inclusion Criteria & Exclusion Criteria \\
\hline $\begin{array}{ll}\text { Study } \\
\text { design }\end{array}$ & - Randomized controlled trials & - Uncontrolled trials \\
& - Non-randomized controlled trials & - Case-control studies \\
including quasi-experimental and & controlled before-and-after studies & - Cross-sectional studies \\
& - Prospective cohort studies & - Uncontrolled before-and-after \\
& - Retrospective cohort studies & - Nadies \\
& - Nested case-control studies & - Systematic reviews \\
& & - Meta-analyses
\end{tabular}

Intervention/ - Dietary intake of a food(s) or food exposure group(s)

\begin{tabular}{|c|c|c|}
\hline Comparator & $\begin{array}{l}\text { Different amount of dietary intake } \\
\text { of the same food(s) or food } \\
\text { group(s) }\end{array}$ & - No comparator \\
\hline Outcomes & $\begin{array}{ll}\text { - } & \text { Food allergies } \\
\text { - } & \text { Atopic dermatitis } \\
\text { - } & \text { Allergic rhinitis } \\
\text { - } & \text { Asthma ( } \geq 2 \text { years old) }\end{array}$ & $\begin{array}{l}\text { Food allergy when diagnosis } \\
\text { was based solely on food } \\
\text { allergen sensitization (i.e., } \\
\text { skin prick test, or serum lgE } \\
\text { measure) (i.e., without an oral } \\
\text { food challenge or parental } \\
\text { report of clinical history) } \\
\text { - Asthma when diagnosis was } \\
\text { based solely on report of } \\
\text { wheeze }\end{array}$ \\
\hline $\begin{array}{l}\text { Date of } \\
\text { publication }\end{array}$ & - January 1980 to January 2020 & $\begin{array}{l}\text { - Articles published before } \\
\text { January } 1980 \text { or after January } \\
2020\end{array}$ \\
\hline
\end{tabular}




\begin{tabular}{|c|c|c|}
\hline Category & Inclusion Criteria & Exclusion Criteria \\
\hline $\begin{array}{l}\text { Publication } \\
\text { status }\end{array}$ & $\begin{array}{l}\text { - Articles that have been peer- } \\
\text { reviewed }\end{array}$ & $\begin{array}{l}\text { - Articles that have not been } \\
\text { peer-reviewed and are not } \\
\text { published in peer-reviewed } \\
\text { journals, including } \\
\text { unpublished data, } \\
\text { manuscripts, reports, } \\
\text { abstracts, and conference } \\
\text { proceedings }\end{array}$ \\
\hline $\begin{array}{l}\text { Language of } \\
\text { publication }\end{array}$ & - Articles published in English & $\begin{array}{l}\text { - Articles published in } \\
\text { languages other than English }\end{array}$ \\
\hline Countryxxiii & $\begin{array}{l}\text { - Studies conducted in countries } \\
\text { ranked as high or very high human } \\
\text { development }\end{array}$ & $\begin{array}{l}\text { - Studies conducted in } \\
\text { countries ranked as medium } \\
\text { or lower human development }\end{array}$ \\
\hline $\begin{array}{l}\text { Study } \\
\text { participants }\end{array}$ & - Human participants & $\begin{array}{l}\text { - Non-human participants (e.g., } \\
\text { animal or in-vitro models) }\end{array}$ \\
\hline $\begin{array}{l}\text { Life stage of } \\
\text { study } \\
\text { participants } \\
\\
\text { intervention } \\
\text { or exposure }\end{array}$ & $\begin{array}{l}\text { - Women during pregnancy OR } \\
\text { women during both pregnancy and } \\
\text { lactation, regardless of human milk } \\
\text { feeding status } \\
\text { - Women during lactation who are } \\
\text { exclusively or predominantly } \\
\text { feeding human milk }\end{array}$ & $\begin{array}{l}\text { - Studies conducted } \\
\text { exclusively in non-lactating } \\
\text { women or in lactating women } \\
\text { who are not exclusively or } \\
\text { predominantly feeding human } \\
\text { milk. }\end{array}$ \\
\hline \multirow[t]{2}{*}{$\begin{array}{l}\text { Life stage of } \\
\text { study } \\
\text { participants } \\
\text { - outcomes: }\end{array}$} & $\begin{array}{l}\text { Food allergies, atopic dermatitis, and } \\
\text { allergic rhinitis: } \\
\text { - Infants and toddlers (birth }-24 \\
\text { months) }\end{array}$ & $\begin{array}{l}\text { Asthma: } \\
\text { - Infants and toddlers (birth - } \\
24 \text { months) }\end{array}$ \\
\hline & $\begin{array}{l}\text { All outcomes: } \\
\text { - Children and adolescents }(2-18 \\
\text { years) }\end{array}$ & $\begin{array}{l}\text { All outcomes: } \\
\text { - Adults (19-64 years) } \\
\text { - Older adults ( } 65 \text { years and } \\
\text { older) }\end{array}$ \\
\hline
\end{tabular}

\footnotetext{
xxiii The Human Development classification was based on the Human Development Index (HDI) ranking from the year the study intervention occurred or data were collected (UN Development Program. HDI 1990-2017 HDRO calculations based on data from UNDESA (2017a), UNESCO Institute for Statistics (2018), United Nations Statistics Division (2018b), World Bank (2018b), Barro and Lee (2016) and IMF (2018). Available from: http://hdr.undp.org/en/data). If the study did not report the year in which the intervention occurred or data were collected, the HDI classification for the year of publication was applied. HDI values are available from 1980, and then from 1990 to present. If a study was conducted prior to 1990, the HDI classification from 1990 was applied. When a country was not included in the HDI ranking, the current country classification from the World Bank was used instead (The World Bank. World Bank country and lending groups. Available from: https://datahelpdesk.worldbank.org/knowledgebase/articles/906519-world- country-and-lending-groups).
} 


\begin{tabular}{|c|c|c|}
\hline Category & Inclusion Criteria & Exclusion Criteria \\
\hline $\begin{array}{l}\text { Health } \\
\text { status of } \\
\text { study } \\
\text { participants }\end{array}$ & $\begin{array}{l}\text { - Studies that enroll participants who } \\
\text { are healthy and/or at risk for } \\
\text { chronic disease, including those } \\
\text { with obesity } \\
\text { - Studies that enroll some children } \\
\text { diagnosed with a disease or with } \\
\text { the health outcome of interest: } \\
\circ \text { Food allergies } \\
\circ \text { Atopic dermatitis } \\
\circ \text { Allergic rhinitis } \\
\circ \text { Asthma }\end{array}$ & $\begin{array}{l}\text { - Studies that exclusively } \\
\text { enroll mothers who gave birth } \\
\text { to preterm (<37 weeks and } \\
0 / 7 \text { days) infants } \\
\text { - Studies that exclusively } \\
\text { enroll mothers diagnosed with } \\
\text { a chronic disease, including } \\
\text { severe undernutrition or } \\
\text { hospitalized with an illness or } \\
\text { injury }\end{array}$ \\
\hline
\end{tabular}

\section{Electronic databases and search terms}

\section{PubMed}

- Provider: U.S. National Library of Medicine

- Date searched: January 21, 2020

- Date range searched: January 1, 1980 - January 21, 2020

- Search terms:

\#1 - Diet [Mesh] OR diet[tiab] OR diets[tiab] OR dietary[tiab] OR food*[tiab] OR foods[tiab] OR "Food"[Mesh] OR "Eating"[Mesh] OR (maternal tiab] AND intake[tiab])

\#2 - (("Allergy and Immunology"[Mesh:NoExp] OR allerg*[tiab] OR Hypersensitiv*[tiab] OR atopic[tiab]) AND (food*[tiab] OR peanut*[tiab] OR nut[tiab] OR nuts[tiab] OR egg*[tiab] OR eggs[tiab] OR milk[tiab] OR shellfish[tiab] OR fish[tiab] OR fishes[Mesh] OR seafood[Mesh] OR wheat[tiab] OR gluten*[tiab] OR glutens[Mesh] OR dairy[tiab] OR dairy products[Mesh] OR eggs[Mesh] OR nuts[Mesh] OR soy foods[mh] OR soy*[tiab])) OR "Food Hypersensitivity"[Mesh] OR asthma*[tiab] OR asthma[Mesh] OR "Rhinitis, Allergic"[Mesh] OR (allerg*[tiab] AND rhiniti*[tiab]) OR "Dermatitis, Atopic"[Mesh] OR atopic dermatitis*[tiab] OR eczema*[tiab] OR eczema[Mesh] OR "Immunoglobulin E"[Mesh] OR "Immunoglobulin E"[tiab] OR IgE[tiab]

\#3 - ("Pregnancy"[Mesh] OR "Pregnancy Complications"[Mesh] OR "Maternal Exposure"[Mesh] OR "pregnant women"[Mesh] OR pregnan*[tiab] OR pre-pregnancy[tiab] OR prenatal[tiab] OR pre-natal[tiab] OR maternal[tiab] OR mother*[tiab] OR "Mothers"[Mesh] OR perinatal[tiab] OR peri-natal[tiab] OR pre-conception[tiab] OR preconception[tiab] OR periconception[tiab] OR periconception[tiab] OR "Peripartum Period"[Mesh] OR peripartum[tiab] OR peri-partum[tiab] OR gestat*[tiab] OR natal[tiab] OR antenatal[tiab] OR ante-natal[tiab] OR puerperium[tiab] OR "Maternal Nutritional Physiological Phenomena"[Mesh] OR "Postpartum Period"[Mesh] OR postpart*[tiab] OR post-part[tiab] OR pre-parturition[tiab] OR postnatal[tiab] OR post-natal[tiab] OR post delivery[tiab] OR postdelivery[tiab] OR after birth[tiab] OR afterbirth[tiab] OR Lactation[Mesh] OR lactati*[tiab] OR breast feeding[Mesh] OR breastfe*[tiab] OR breast fe*[tiab] OR Milk, Human [Mesh] OR breastmilk[tiab] OR "breast 
milk"[tiab] OR "human milk"[tiab] OR "maternal milk"[tiab] OR "nursing women"[tiab]))

\#4 - \#1 AND \#2 AND \#3

\#5 - \#4 NOT ("Animals"[Mesh] NOT ("Animals"[Mesh] AND "Humans"[Mesh])) NOT (editorial[ptyp] OR comment[ptyp] OR news[ptyp] OR letter[ptyp] OR review[ptyp] OR systematic review[ptyp] OR systematic review[ti] OR meta-analysis[ptyp] OR meta-analysis[ti] OR meta-analyses[ti] OR retracted publication[ptyp] OR retraction of publication[ptyp] OR retraction of publication[tiab] OR retraction notice[ti])

Filters: Publication date from 1980/01/01 to 2020/01/21; English

\section{Cochrane Central Register of Controlled Trials (CENTRAL)}

- Provider: John Wiley \& Sons

- Date searched: January 21, 2020

- Date range searched: January 1, 1980 - January 21, 2020

- Search terms:

\#1 - [mh "Diet"] OR diet OR diets OR dietary OR food* OR foods OR [mh "Food"] OR [mh "Eating"] OR (maternal NEAR/4 intake)

\#2 - [mh ^"Allergy and Immunology"] OR ((allerg* OR Hypersensitivit*) NEAR/4 (food* OR peanut* OR nut OR nuts OR egg* OR milk OR shellfish OR seafood* OR wheat OR gluten* OR dairy OR fish OR soy*)) OR [mh "Food Hypersensitivity"] OR asthma* OR [mh "Rhinitis, Allergic"] OR (allerg* NEAR/4 Rhiniti*) OR [mh "Dermatitis, Atopic"] OR ((Dermatiti* OR eczema) NEAR/4 Atopic) OR (Infant* NEAR/4 Eczema) OR [mh "Immunoglobulin E"] OR "Immunoglobulin E"

\#3 - [mh "Pregnancy"] OR [mh "Pregnancy Complications"] OR [mh "Prenatal Exposure Delayed Effects"] OR [mh "Maternal Exposure"] OR [mh "Pregnant Women"] OR [mh "Mothers"] OR [mh "Peripartum Period"] OR [mh "Maternal Nutritional Physiological Phenomena"] OR [mh "Postpartum Period"] OR [mh Lactation] OR [mh "Breast Feeding"] OR [mh "Milk, Human"] OR (pregnancy OR pre-pregnancy OR prenatal OR pre-natal OR maternal OR mother ${ }^{*}$ OR postpartum OR perinatal OR peri-natal OR pre-conception OR preconception OR peri-conception OR periconception OR peripartum OR peri-partum OR gestat* OR natal OR antenatal OR ante-natal OR puerperium OR postpartum OR post-partum OR perinatal OR peri-natal OR puerperium OR postpartal OR post-partal OR postnatal OR "post delivery" OR "after birth" OR lactation OR lactating OR breastfeeding OR breast-feeding OR breast feed" OR breast-feed* OR breastfed OR breast-fed OR breastfeed OR "human milk" OR "nursing women"):ti,ab,kw

\#4 - \#1 AND \#2 AND \#3

Filters: Publication Year from 1980 to 2020, in Trials 


\section{Embase}

- Provider: Elsevier

- Date searched: January 21, 2020

- Date range searched: January 1, 1980 - January 21, 2020

- Search terms:

\#1 - 'diet'/exp OR 'food'/exp OR 'eating'/exp OR diet:ti,ab OR diets:ti,ab OR dietary:ti,ab OR food:ti,ab OR foods:ti,ab OR (maternal NEAR/4 intake*) OR eating:ti,ab OR eat:ti,ab

\#2 - 'allergic asthma'/exp OR 'food allergy'/exp OR 'allergic rhinitis'/exp OR 'dermatitis'/exp OR 'eczema'/exp OR 'skin allergy'/exp OR ((allerg* OR hypersensitivity*) NEAR/4 (food OR peanut* OR nut OR nuts OR egg OR eggs OR milk OR shellfish OR seafood* OR wheat OR gluten* OR fish OR dairy OR soy*)) OR 'immunoglobulin e'/exp OR 'immunoglobulin e':ti,ab

\#3 - pregnancy:ab,ti OR 'pre pregnancy':ab,ti OR prenatal:ab,ti OR 'pre natal':ab,ti OR maternal:ab,ti OR mother:ab,ti OR mothers:ab,ti OR 'pre conception':ab,ti OR preconception:ab,ti OR 'peri conception':ab,ti OR periconception:ab,ti OR peripartum:ab,ti OR 'peri partum':ab,ti OR gestation*:ab,ti OR natal:ab,ti OR antenatal:ab,ti OR 'ante natal':ab,ti OR postpartum:ab,ti OR post-partum:ab,ti OR perinatal:ab,ti OR 'peri natal':ab,ti OR puerperium:ab,ti OR postpartal:ab,ti OR post-partal:ab,ti OR postnatal:ab,ti OR 'post delivery':ab,ti OR 'after birth':ab,ti OR lactation:ab,ti OR lactating:ab,ti OR breastfeeding:ab,ti OR breast-feeding:ab,ti OR 'breast feed"':ab,ti OR breastfed:ab,ti OR 'breast fed':ab,ti OR breastfeed:ab,ti OR 'human milk':ab,ti OR 'nursing women':ab,ti OR 'pregnancy'/exp/mj OR 'pregnancy complication'/exp/mj OR 'prenatal exposure'/mj OR 'maternal exposure'/mj OR 'pregnant woman'/mj OR 'mother'/mj OR 'puerperium'/exp/mj OR 'maternal nutrition'/mj OR 'lactation'/mj OR 'breast feeding'/exp/mj OR 'breast milk'/exp/mj

\#4 - \#1 AND \#2 AND \#3

\#5 - \#4 AND ([article]/lim OR [article in press]/lim) AND [humans]/lim AND [english]/lim AND [1980-2020]/py NOT ([conference abstract]/lim OR [conference paper]/lim OR [editorial]/lim OR [erratum]/lim OR [letter]/lim OR [note]/lim OR [review]/lim OR [systematic review]/lim OR [meta analysis]/lim)

\section{Cumulative Index of Nursing and Allied Health Literature (CINAHL Plus)}

- Provider: EBSCOhost

- Date searched: January 21, 2020

- Date range searched: January 1, 1980 - January 21, 2020

- Search terms:

\#1 - (mh "Diet") OR diet OR diets OR dietary OR food OR foods[tiab] OR (mh "Food") OR (mh "Eating") OR (maternal N4 intake*)

\#2 - (mh "Allergy and Immunology") OR ((allerg* OR Hypersensitivit*) N4 (food* OR peanut ${ }^{*}$ OR nut OR nuts OR egg* OR milk OR shellfish OR seafood OR wheat OR gluten* OR dairy OR fish OR soy*)) OR (mh "Food Hypersensitivity+") OR asthma* OR (mh "Rhinitis, Allergic") OR (allerg* N4 Rhiniti*) OR (mh "Dermatitis, Atopic") OR ((Dermatiti* OR eczema) N4 Atopic)) OR (Infant* N5 Eczema) OR (mh "Immunoglobulin E") OR "Immunoglobulin E" 
\#3 - postpartum OR post-partum OR MH "Postpartum Period" OR postpartal OR post-partal OR postnatal OR post-natal OR "post deliver*" OR "after birth" OR MH pregnancy OR $\mathrm{MH}$ "pregnancy complications" OR MH "Prenatal Exposure Delayed Effects" OR MH "Maternal Exposure" OR MH "pregnant women" OR pregnan* OR pre-pregnancy OR prepregnancy OR prenatal OR antenatal OR maternal OR mother OR mothers OR perinatal OR peri-natal OR peri-conception OR periconception OR MH "Peripartum Period" OR peripartum OR peripartum OR gestation* OR natal OR puerperium OR MH "Maternal Nutritional Physiological Phenomena" OR MH "Breast Feeding"OR breastfeeding OR breast-feeding OR MH "Milk, Human" OR "human milk" OR MH Lactation OR lactation OR lactating OR breastfeeding OR "breast feed*" OR breast-feed* OR breastfed OR breast-fed OR breastfeed* OR "nursing women" OR "nursing mother*"

\#4 - \#1 AND \#2 AND \#3

\#5 - \#4 NOT (MH "Literature Review" OR MH "Meta Analysis" OR MH "Systematic Review" OR MH "News" OR MH "Retracted Publication" OR MH "Retraction of Publication)

Filters: Published Date: 19800101 - 20200121, English Language, Human

\section{LITERATURE SEARCH AND SCREENING RESULTS}

The flow chart (Figure 2) below illustrates the literature search and screening results for articles examining the systematic review question. The results of the electronic database searches, after removal of duplicates, were screened independently by two NESR analysts using a step-wise process by reviewing titles, abstracts, and full-texts to determine which articles met the inclusion criteria. Refer to Table $\mathbf{1 0}$ for the rationale for exclusion for each excluded full-text article. A manual search was done to find articles that were not identified when searching the electronic databases; all manually identified articles are also screened to determine whether they meet criteria for inclusion. 
Figure 2: Flow chart of literature search and screening results

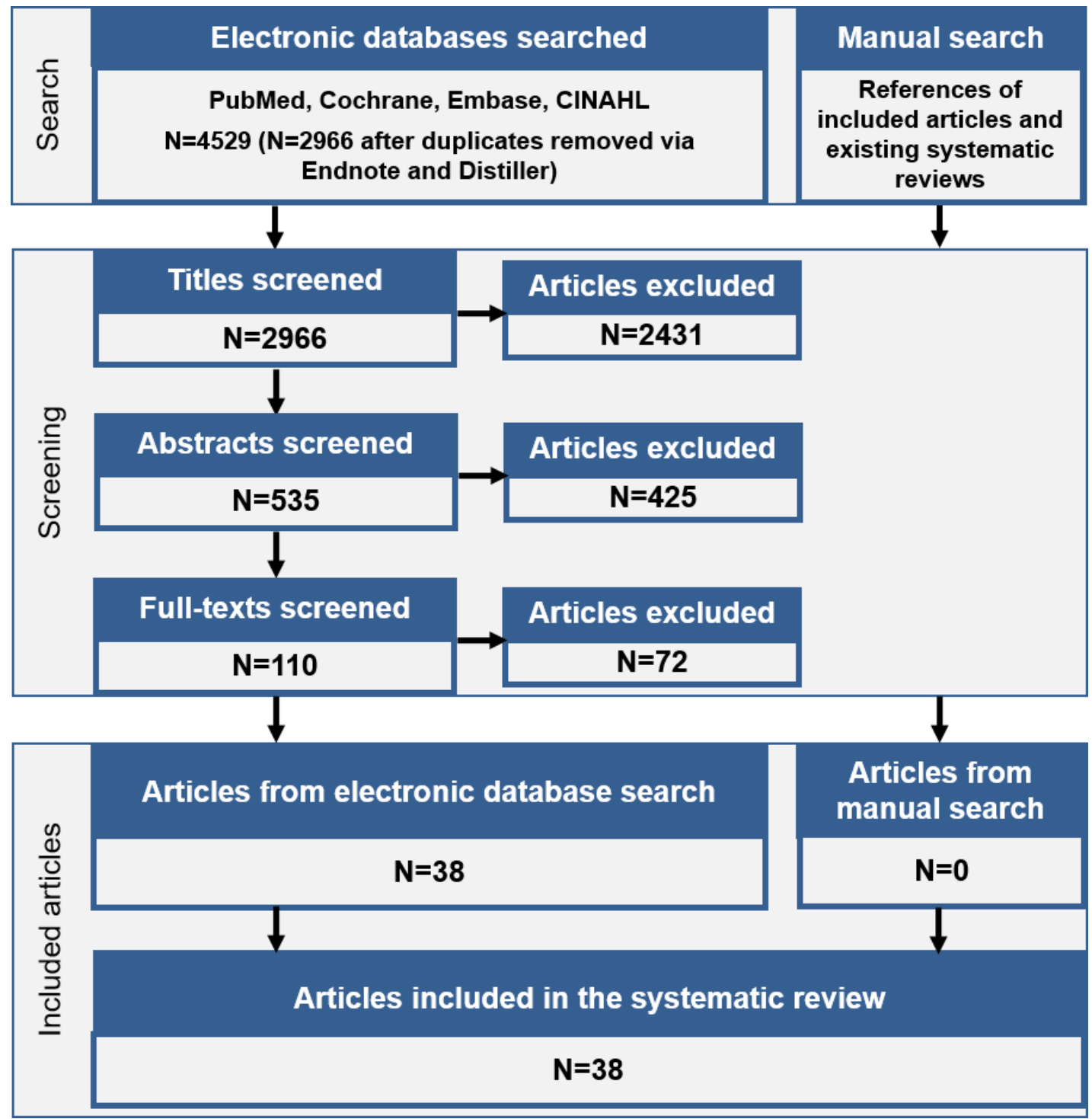




\section{Excluded articles}

The table below lists the articles excluded after full-text screening, and includes columns for the categories of inclusion and exclusion criteria (see Table 9) that studies were excluded based on. At least one reason for exclusion is provided for each article, though this may not reflect all possible reasons for exclusion. Information about articles excluded after title and abstract screening is available upon request.

\section{Table 10. Articles excluded after full text screening with rationale for exclusion}

$\begin{array}{ll}\text { Citation Rationale } & \end{array}$

1. Abrams, EM, Sicherer, SH. Maternal peanut consumption and risk of peanut allergy in childhood. Cmaj. 2018. 190:E814-e815. doi:10.1503/cmaj.180563.

2. Alvarez Zallo, N, Aguinaga-Ontoso, I, Alvarez-Alvarez, I, Marin-Fernandez, B, Guillen-Grima, $\mathrm{F}$, Azcona-San Julian, C. Influence of the Mediterranean diet during pregnancy in the development of wheezing and eczema in infants in Pamplona, Spain. Allergol Immunopathol (Madr). 2018. 46:9-14. doi:10.1016/j.aller.2017.02.009.

3. Arshad, SH, Bateman, B, Sadeghnejad, A, Gant, C, Matthews, SM. Prevention of allergic disease during childhood by allergen avoidance: the Isle of Wight prevention study. J Allergy Clin Immunol. 2007. 119:307-13. doi:10.1016/j.jaci.2006.12.621.

4. Baiz, N, Just, J, Chastang, J, Forhan, A, de Lauzon-Guillain, B, Magnier, AM, AnnesiMaesano, I. Maternal diet before and during pregnancy and risk of asthma and allergic rhinitis in children. Allergy Asthma Clin Immunol. 2019. 15:40. doi:10.1186/s13223-0190353-2.

5. Bedard, A, Northstone, K, Henderson, AJ, Shaheen, SO. Maternal intake of sugar during pregnancy and childhood respiratory and atopic outcomes. Eur Respir J. 2017. 50. doi:10.1183/13993003.00073-2017.

6. Berdi, M, de Lauzon-Guillain, B, Forhan, A, Castelli, FA, Fenaille, F, Charles, MA, Heude, B,

Study design; Outcome Junot, C, Adel-Patient, K. Immune components of early breastmilk: Association with maternal factors and with reported food allergy in childhood. Pediatr Allergy Immunol. 2019. 30:107-116. doi:10.1111/pai.12998.

7. Binkley, KE, Leaver, C, Ray, JG. Antenatal risk factors for peanut allergy in children. Allergy Asthma Clin Immunol. 2011. 7:17. doi:10.1186/1710-1492-7-17.

8. Bisgaard, H, Stokholm, J, Chawes, BL, Vissing, NH, Bjarnadottir, E, Schoos, AM, Wolsk, HM, Pedersen, TM, Vinding, RK, Thorsteinsdottir, S, Folsgaard, NV, Fink, NR, Thorsen, J, Pedersen, AG, Waage, J, Rasmussen, MA, Stark, KD, Olsen, SF, Bonnelykke, K. Fish OilDerived Fatty Acids in Pregnancy and Wheeze and Asthma in Offspring. N Engl J Med. 2016. 375:2530-9. doi:10.1056/NEJMoa1503734. 


\section{Citation}

9.

Calvani, M, Alessandri, C, Sopo, SM, Panetta, V, Pingitore, G, Tripodi, S, Zappala, D, Zicari,

AM. Consumption of fish, butter and margarine during pregnancy and development of allergic sensitizations in the offspring: role of maternal atopy. Pediatr Allergy Immunol. 2006.

17:94-102. doi:10.1111/j.1399-3038.2005.00367.x.

10. Castro-Rodriguez, JA, Garcia-Marcos, L, Sanchez-Solis, M, Perez-Fernandez, V, MartinezTorres, A, Mallol, J. Olive oil during pregnancy is associated with reduced wheezing during the first year of life of the offspring. Pediatr Pulmonol. 2010. 45:395-402. doi:10.1002/ppul.21205.

11. Castro-Rodriguez, JA, Ramirez-Hernandez, M, Padilla, O, Pacheco-Gonzalez, RM, PerezFernandez, V, Garcia-Marcos, L. Effect of foods and Mediterranean diet during pregnancy and first years of life on wheezing, rhinitis and dermatitis in preschoolers. Allergo Immunopathol (Madr). 2016. 44:400-9. doi:10.1016/j.aller.2015.12.002.

12. Celik, V, Beken, B, Yazicioglu, M, Ozdemir, PG, Sut, N. Do traditional fermented foods protect against infantile atopic dermatitis. Pediatr Allergy Immunol. 2019. 30:540-546. doi:10.1111/pai.13045.

13. Chandra, RK, Puri, S, Suraiya, C, Cheema, PS. Influence of maternal food antigen avoidance during pregnancy and lactation on incidence of atopic eczema in infants. Clin Allergy. 1986. 16:563-9. doi:10.1111/j.1365-2222.1986.tb01995.x.

14. Chapman, DJ. Does maternal diet contribute to colic among breastfed infants?. J Hum Lact. Study design; Outcome 2006. 22:236-7. doi:10.1177/0890334406287108.

15. Chatzi, L, Torrent, M, Romieu, I, Garcia-Esteban, R, Ferrer, C, Vioque, J, Kogevinas, M Sunyer, J. Mediterranean diet in pregnancy is protective for wheeze and atopy in childhood. Thorax. 2008. 63:507-13. doi:10.1136/thx.2007.081745.

16. Dean, T, Venter, C, Pereira, B, Grundy, J, Clayton, CB, Higgins, B. Government advice on peanut avoidance during pregnancy--is it followed correctly and what is the impact on sensitization?. J Hum Nutr Diet. 2007. 20:95-9. doi:10.1111/j.1365-277X.2007.00751.x.

17. Dotterud, CK, Storro, O, Simpson, MR, Johnsen, $R$, Oien, T. The impact of pre- and postnatal exposures on allergy related diseases in childhood: a controlled multicentre intervention study in primary health care. BMC Public Health. 2013. 13:123. doi:10.1186/1471-2458-13-123.

18. Dubakiene, R, Rudzeviciene, O, Butiene, I, Sezaite, I, Petronyte, M, Vaicekauskaite, D, Zvirbliene, A. Studies on early allergic sensitization in the Lithuanian birth cohort. ScientificWorldJournal. 2012. 2012:909524. doi:10.1100/2012/909524.

\section{Rationale}

Study design

Study design; Population

Study design; Intervention/Exposure

Study design

Retracted

Outcome

Intervention/Exposure; Outcome

Study design; Comparator; Cross-sectional data for control cohort

Exposure-outcome analysis not clear, data not reported 


\section{Citation}

19. Erkkola, M, Nwaru, BI, Kaila, M, Kronberg-Kippila, C, Ilonen, J, Simell, O, Veijola, R, Knip, $\mathrm{M}$, Virtanen, SM. Risk of asthma and allergic outcomes in the offspring in relation to maternal food consumption during pregnancy: a Finnish birth cohort study. Pediatr Allergy Immunol. 2012. 23:186-94. doi:10.1111/j.1399-3038.2012.01272.x.

20. Erratum: effect of combined maternal and infant food-allergen avoidance on development of atopy in early infancy: a randomized study (Zeiger et al (1989) 84 (72)). Journal of allergy and clinical immunology. 1989. 84:677-.

21. Falth-Magnusson, $\mathrm{K}, \mathrm{Kjellman}, \mathrm{NI}$. Allergy prevention by maternal elimination diet during late pregnancy--a 5-year follow-up of a randomized study. J Allergy Clin Immunol. 1992. 89:70913. doi:10.1016/0091-6749(92)90378-f.

22. Falth-Magnusson, K. Breast milk antibodies to foods in relation to maternal diet, maternal atopy and the development of atopic disease in the baby. Int Arch Allergy Appl Immunol. 1989. 90:297-300. doi:10.1159/000235041.

23. Fitzsimon, N, Fallon, U, O'Mahony, D, Loftus, BG, Bury, G, Murphy, AW, Kelleher, CC. Mothers' dietary patterns during pregnancy and risk of asthma symptoms in children at 3 years. Ir Med J. 2007. 100:suppl 27-32.

24. Frazier, AL, Camargo, CA, Jr, Malspeis, S, Willett, WC, Young, MC. Prospective study of peripregnancy consumption of peanuts or tree nuts by mothers and the risk of peanut or tree nut allergy in their offspring. JAMA Pediatr. 2014. 168:156-62. doi:10.1001/jamapediatrics.2013.4139.

25. Fukuda, Y, Yamamoto, S, Kameda, M. Association Between Lifestyle Characteristics and Body Mass Index of Mothers of Children With Allergic Diseases. J Clin Med Res. 2019. 11:780-788. doi:10.14740/jocmr3976.

26. Gao, X, Yan, Y, Zeng, G, Sha, T, Liu, S, He, Q, Chen, C, Li, L, Xiang, S, Li, H, Tan, S, Yan, Q. Influence of prenatal and early-life exposures on food allergy and eczema in infancy: a birth cohort study. BMC Pediatr. 2019. 19:239. doi:10.1186/s12887-019-1623-3.

27. Hansen, S, Strom, M, Maslova, E, Dahl, R, Hoffmann, HJ, Rytter, D, Bech, BH, Henriksen, TB, Granstrom, C, Halldorsson, TI, Chavarro, JE, Linneberg, A, Olsen, SF. Fish oil supplementation during pregnancy and allergic respiratory disease in the adult offspring. $J$ Allergy Clin Immunol. 2017. 139:104-111.e4. doi:10.1016/j.jaci.2016.02.042.

28. Harris, JM, Cullinan, $P$, Williams, HC, Mills, $P$, Moffat, S, White, C, Newman Taylor, AJ. Environmental associations with eczema in early life. Br J Dermatol. 2001. 144:795-802. doi:10.1046/j.1365-2133.2001.04135.x.

29. Hattevig, G, Kjellman, B, Sigurs, N, Bjorksten, B, Kjellman, NI. Effect of maternal avoidance of eggs, cow's milk and fish during lactation upon allergic manifestations in infants. Clin Exp Allergy. 1989. 19:27-32. doi:10.1111/j.1365-2222.1989.tb02339.x.

\section{Rationale}

Study design; Diet assessed after birth

Erratum

Outcome

Outcome

Study design; Population

Population

Study design; Health Status

Study design; Diet assessed after birth

Intervention/Exposure;

Intervention/Exposure; Population

Population; Human milk feeding practices unclear 


\section{Citation}

30. Hattevig, G, Kjellman, B, Sigurs, N, Grodzinsky, E, Hed, J, Bjorksten, B. The effect of maternal avoidance of eggs, cow's milk, and fish during lactation on the development of $\operatorname{lgE}$, IgG, and IgA antibodies in infants. J Allergy Clin Immunol. 1990. 85:108-15. doi:10.1016/0091-6749(90)90231-r.

31. Hattevig, G, Sigurs, N, Kjellman, B. Effects of maternal dietary avoidance during lactation on allergy in children at 10 years of age. Acta Paediatr. 1999. 88:7-12.

32. Hoppu, U, Kalliomaki, M, Isolauri, E. Maternal diet rich in saturated fat during breastfeeding is associated with atopic sensitization of the infant. Eur J Clin Nutr. 2000. 54:702-5. doi:10.1038/sj.ejcn.1601079.

33. Jonsson, K, Barman, M, Moberg, S, Sjoberg, A, Brekke, HK, Hesselmar, B, Johansen, S, Wold, AE, Sandberg, AS. Fat intake and breast milk fatty acid composition in farming and nonfarming women and allergy development in the offspring. Pediatr Res. 2016. 79:114-23. doi:10.1038/pr.2015.187.

34. Jonsson, K, Barman, M, Moberg, S, Sjoberg, A, Brekke, HK, Hesselmar, B, Sandberg, AS, Wold, AE. Serum fatty acids in infants, reflecting family fish consumption, were inversely associated with allergy development but not related to farm residence. Acta Paediatr. 2016. 105:1462-1471. doi:10.1111/apa.13592.

35. Kelleher, CC, Viljoen, K, Khalil, H, Somerville, R, O'Brien, J, Shrivastava, A, Murrin, C Longitudinal follow-up of the relationship between dietary intake and growth and

development in the Lifeways cross-generation cohort study 2001-2013. Proc Nutr Soc. 2014. 73:118-31. doi:10.1017/s002966511300373x.

36. Kemp, AS, Ponsonby, AL, Dwyer, T, Cochrane, JA, Pezic, A, Jones, G. Maternal antenatal peanut consumption and peanut and rye sensitization in the offspring at adolescence. Clin Exp Allergy. 2011. 41:224-31. doi:10.1111/j.1365-2222.2010.03668.x.

37. Kim, YH, Kim, KW, Lee, SY, Koo, KO, Kwon, SO, Seo, JH, Suh, DI, Shin, YH, Ahn, K, Oh SY, Lee, S, Sohn, MH, Hong, SJ. Maternal Perinatal Dietary Patterns Affect Food Allergy Development in Susceptible Infants. J Allergy Clin Immunol Pract. 2019. 7:2337-2347.e7. doi:10.1016/j.jaip.2019.03.026.

38. Kusmierek, M, Sardecka, I, Los-Rycharska, E, Krogulska, A. The impact of immunomodulatory factors from maternal diet during pregnancy on cow's milk allergy in offspring - A pilot study in the paediatric population of the Kuyavian-Pomeranian Voivodship. Allergol Immunopathol (Madr). 2019. 47:570-578. doi:10.1016/j.aller.2019.04.007.

39. Levin, ME, Botha, M, Basera, W, Facey-Thomas, HE, Gaunt, B, Gray, CL, Kiragu, W, Ramjith, J, Watkins, A, Genuneit, J. Environmental factors associated with allergy in urban and rural children from the South African Food Allergy (SAFFA) cohort. J Allergy Clin Immunol. 2020. 145:415-426. doi:10.1016/j.jaci.2019.07.048.

\section{Rationale}

Outcome

Population; Human milk feeding practices unclear

Intervention/Exposure;

Outcome; Composite outcome

Intervention/Exposure; Outcome; Exp-outcome not tested

\section{Study design}

\section{Study design; Outcome}

Outcome; Did not require skin prick test; No obvious food challenge

Study design; Health Status

Study design; Population 


\section{Citation}

40. Lilja, G, Dannaeus, A, Foucard, T, Graff-Lonnevig, V, Johansson, SG, Oman, H. Effects of maternal diet during late pregnancy and lactation on the development of IgE and egg- and milk-specific IgE and IgG antibodies in infants. Clin Exp Allergy. 1991. 21:195-202. doi:10.1111/j.1365-2222.1991.tb00830.x.

41. Linnamaa, P, Nieminen, K, Koulu, L, Tuomasjukka, S, Kallio, H, Yang, B, Tahvonen, R, Savolainen, J. Black currant seed oil supplementation of mothers enhances IFN-gamma and suppresses IL-4 production in breast milk. Pediatr Allergy Immunol. 2013. 24:562-6. doi:10.1111/pai.12110.

42. Linnamaa, P, Savolainen, J, Koulu, L, Tuomasjukka, S, Kallio, H, Yang, B, Vahlberg, T, Tahvonen, R. Blackcurrant seed oil for prevention of atopic dermatitis in newborns: a randomized, double-blind, placebo-controlled trial. Clin Exp Allergy. 2010. 40:1247-55. doi:10.1111/j.1365-2222.2010.03540.x.

43. Losol, P, Rezwan, FI, Patil, VK, Venter, C, Ewart, S, Zhang, H, Arshad, SH, Karmaus, W, Holloway, JW. Effect of gestational oily fish intake on the risk of allergy in children may be influenced by FADS1/2, ELOVL5 expression and DNA methylation. Genes Nutr. 2019. 14:20. doi:10.1186/s12263-019-0644-8.

44. Ludvigsson, J. Cow-milk-free diet during last trimester of pregnancy does not influence diabetes-related autoantibodies in nondiabetic children. Ann N Y Acad Sci. 2003. 1005:2758. doi:10.1196/annals.1288.042.

45. Lumia, M, Luukkainen, $\mathrm{P}$, Tapanainen, H, Kaila, M, Erkkola, M, Uusitalo, L, Niinisto, S, Kenward, MG, llonen, J, Simell, O, Knip, M, Veijola, R, Virtanen, SM. Dietary fatty acid composition during pregnancy and the risk of asthma in the offspring. Pediatr Allergy Immunol. 2011. 22:827-35. doi:10.1111/j.1399-3038.2011.01202.x.

46. Martin-Munoz, MF, Pineda, F, Garcia Parrado, G, Guillen, D, Rivero, D, Belver, T, Quirce, S. Food allergy in breastfeeding babies. Hidden allergens in human milk. Eur Ann Allergy Clin Immunol. 2016. 48:123-8.

47. Miyake, Y, Tanaka, K, Yasutake, A, Sasaki, S, Hirota, Y. Lack of association of mercury with risk of wheeze and eczema in Japanese children: the Osaka Maternal and Child Health Study. Environ Res. 2011. 111:1180-4. doi:10.1016/j.envres.2011.07.003.

48. Nascimento, JXPT, Ribeiro, CCC, Batista, RFL, de Britto Alves, MTSS, Simoes, VMF, Padilha, LL, Cardoso, VC, Vianna, EO, Bettiol, H, Barbieri, MA, Silva, AAMD. The First 1000 Days of Life Factors Associated with "Childhood Asthma Symptoms": Brisa Cohort, Brazil. Sci Rep. 2017. 7:16028. doi:10.1038/s41598-017-16295-4.

49. Neild, V. Diet and atopic eczema. Mod Midwife. 1994. 4:22

50. Ngamphaiboon, J, Chatchatee, P, Thongkaew, T. Cow's milk allergy in Thai children. Asian Pac J Allergy Immunol. 2008. 26:199-204.

\section{Rationale}

Outcome

Intervention/Exposure; Outcome

Intervention/Exposure

Intervention/Exposure; Outcome; No exp-outcome test

\section{Outcome}

Study design; Diet assessed after birth

Study design

Intervention/Exposure;

Outcome; <2y old

Study design; Not a study

Country; Health Status 


\section{Citation}

51. Nwaru, BI, Ahonen, S, Kaila, M, Erkkola, M, Haapala, AM, Kronberg-Kippila, C, Veijola, R, Ilonen, J, Simell, O, Knip, M, Virtanen, SM. Maternal diet during pregnancy and allergic sensitization in the offspring by 5 yrs of age: a prospective cohort study. Pediatr Allergy Immunol. 2010. 21:29-37. doi:10.1111/j.1399-3038.2009.00949.x.

52. Nwaru, BI, Erkkola, M, Ahonen, S, Kaila, M, Lumia, M, Prasad, M, Haapala, AM, KronbergKippila, C, Veijola, R, Ilonen, J, Simell, O, Knip, M, Virtanen, SM. Maternal diet during lactation and allergic sensitization in the offspring at age of 5. Pediatr Allergy Immunol. 2011. 22:334-41. doi:10.1111/j.1399-3038.2010.01114.x.

53. Nwaru, BI, Erkkola, M, Lumia, M, Kronberg-Kippila, C, Ahonen, S, Kaila, M, Ilonen, J, Simell, O, Knip, M, Veijola, R, Virtanen, SM. Maternal intake of fatty acids during pregnancy and allergies in the offspring. Br J Nutr. 2012. 108:720-32. doi:10.1017/s0007114511005940.

54. Oien, $\mathrm{T}$, Storro, $\mathrm{O}$, Johnsen, $\mathrm{R}$. Do early intake of fish and fish oil protect against eczema and doctor-diagnosed asthma at 2 years of age? A cohort study. J Epidemiol Community Health. 2010. 64:124-9. doi:10.1136/jech.2008.084921.

55. Olsen, SF, Osterdal, ML, Salvig, JD, Mortensen, LM, Rytter, D, Secher, NJ, Henriksen, TB. Fish oil intake compared with olive oil intake in late pregnancy and asthma in the offspring: 16 y of registry-based follow-up from a randomized controlled trial. Am J Clin Nutr. 2008. 88:167-75. doi:10.1093/ajcn/88.1.167.

56. Ozawa, N, Shimojo, N, Suzuki, Y, Ochiai, S, Nakano, T, Morita, Y, Inoue, Y, Arima, T, Suzuki, S, Kohno, Y. Maternal intake of Natto, a Japan's traditional fermented soybean food, during pregnancy and the risk of eczema in Japanese babies. Allergol Int. 2014. 63:261-6 doi:10.2332/allergolint.13-OA-0613.

57. Pele, F, Bajeux, E, Gendron, H, Monfort, C, Rouget, F, Multigner, L, Viel, JF, Cordier, S. Maternal fish and shellfish consumption and wheeze, eczema and food allergy at age two: a prospective cohort study in Brittany, France. Environ Health. 2013. 12:102. doi:10.1186/1476-069x-12-102.

58. Romieu, I, Torrent, M, Garcia-Esteban, R, Ferrer, C, Ribas-Fito, N, Anto, JM, Sunyer, J. Maternal fish intake during pregnancy and atopy and asthma in infancy. Clin Exp Allergy. 2007. 37:518-25. doi:10.1111/j.1365-2222.2007.02685.x.

59. Salam, MT, Li, YF, Langholz, B, Gilliland, FD. Maternal fish consumption during pregnancy and risk of early childhood asthma. J Asthma. 2005. 42:513-8. doi:10.1081/jas-67619.

60. Sausenthaler, S, Koletzko, S, Schaaf, B, Lehmann, I, Borte, M, Herbarth, O, von Berg, A, Wichmann, HE, Heinrich, J. Maternal diet during pregnancy in relation to eczema and allergic sensitization in the offspring at $2 \mathrm{y}$ of age. Am J Clin Nutr. 2007. 85:530-7. doi:10.1093/ajen/85.2.530.

\section{Rationale}

Outcome

Outcome

Study design; Diet assessed after birth

Study design; Population

Intervention/Exposure;

Diet assessed after birth

Population; Diet assessed before pregnancy

Study design; Population

Study design; Diet during pregnancy assessed after diagnosis

Study design; Diet assessed after birth 


\section{Citation}

61. Sewell, DA, Hammersley, VS, Robertson, A, Devereux, G, Stoddart, A, Weir, CJ, Worth, A, Sheikh, A. A pilot randomised controlled trial investigating a Mediterranean diet intervention in pregnant women for the primary prevention of allergic diseases in infants. J Hum Nutr Diet. 2017. 30:604-614. doi:10.1111/jhn.12469.

62. Sicherer, SH, Wood, RA, Stablein, D, Lindblad, R, Burks, AW, Liu, AH, Jones, SM Fleischer, DM, Leung, DYM, Sampson, HA. Maternal consumption of peanut during pregnancy is associated with peanut sensitization in atopic infants. Journal of Allergy and Clinical Immunology. 2010. 126:1191-1197. doi:10.1016/j.jaci.2010.08.036.

63. Sigurs, N, Hattevig, G, Kjellman, B. Maternal avoidance of eggs, cow's milk, and fish during lactation: effect on allergic manifestations, skin-prick tests, and specific IgE antibodies in children at age 4 years. Pediatrics. 1992. 89:735-9.

64. Stenius, F, Swartz, J, Lilja, G, Borres, M, Bottai, M, Pershagen, G, Scheynius, A, Alm, J. Lifestyle factors and sensitization in children - the ALADDIN birth cohort. Allergy. 2011. 66:1330-8. doi:10.1111/j.1398-9995.2011.02662.x.

65. Storro, O, Oien, T, Dotterud, CK, Jenssen, JA, Johnsen, R. A primary health-care intervention on pre- and postnatal risk factor behavior to prevent childhood allergy. The Prevention of Allergy among Children in Trondheim (PACT) study. BMC Public Health. 2010. 10:443. doi:10.1186/1471-2458-10-443.

66. Stratakis, N, Roumeliotaki, T, Oken, E, Ballester, F, Barros, H, Basterrechea, M, Cordier, S, de Groot, R, den Dekker, HT, Duijts, L, Eggesbo, M, Fantini, MP, Forastiere, F, Gehring, U, Gielen, M, Gori, D, Govarts, E, Inskip, HM, Iszatt, N, Jansen, M, Kelleher, C, Mehegan, J, Molto-Puigmarti, C, Mommers, M, Oliveira, A, Olsen, SF, Pele, F, Pizzi, C, Porta, D, Richiardi, L, Rifas-Shiman, SL, Robinson, SM, Schoeters, G, Strom, M, Sunyer, J, Thijs, C, Vrijheid, M, Vrijkotte, TGM, Wijga, AH, Kogevinas, M, Zeegers, MP, Chatzi, L. Fish and seafood consumption during pregnancy and the risk of asthma and allergic rhinitis in childhood: a pooled analysis of 18 European and US birth cohorts. Int J Epidemiol. 2017. 46:1465-1477. doi:10.1093/ije/dyx007.

67. Tuokkola, J, Luukkainen, $\mathrm{P}$, Tapanainen, H, Kaila, M, Vaarala, O, Kenward, MG, Virta, LJ, Veijola, R, Simell, O, llonen, J, Knip, M, Virtanen, SM. Maternal diet during pregnancy and lactation and cow's milk allergy in offspring. Eur J Clin Nutr. 2016. 70:554-9. doi:10.1038/ejcn.2015.223.

68. Ushiyama, Y, Matsumoto, K, Shinohara, M, Wakiguchi, H, Sakai, K, Komatsu, T, Yamamoto, S. Nutrition during pregnancy may be associated with allergic diseases in infants. J Nutr Sci Vitaminol (Tokyo). 2002. 48:345-51. doi:10.3177/jnsv.48.345.

\section{Rationale}

Outcome

Study design; Health Status; Diet during pregnancy assessed retrospectively

Population; Human milk feeding practices unclear

Intervention/Exposure; No results reported on association btw diet and sensitization

\section{Outcome}

Study design; Meta-analysis

Study design; Population; Outcome

Study design; Intervention/Exposure; Outcome; Diet assessed retrospectively 


\section{Citation}

69. Vance, GH, Grimshaw, KE, Briggs, R, Lewis, SA, Mullee, MA, Thornton, CA, Warner, JO Serum ovalbumin-specific immunoglobulin $G$ responses during pregnancy reflect maternal intake of dietary egg and relate to the development of allergy in early infancy. Clin Exp Allergy. 2004. 34:1855-61. doi:10.1111/j.1365-2222.2004.02111.x.

70. Venter, C, Pereira, B, Voigt, K, Grundy, J, Clayton, CB, Higgins, B, Arshad, SH, Dean, T. Factors associated with maternal dietary intake, feeding and weaning practices, and the development of food hypersensitivity in the infant. Pediatr Allergy Immunol. 2009. 20:320-7. doi:10.1111/j.1399-3038.2008.00832.x.

71. von Ehrenstein, OS, Aralis, H, Flores, ME, Ritz, B. Fast food consumption in pregnancy and subsequent asthma symptoms in young children. Pediatr Allergy Immunol. 2015. 26:571-7. doi:10.1111/pai.12433.

72. Warstedt, K, Furuhjelm, C, Falth-Magnusson, K, Fageras, M, Duchen, K. High levels of omega-3 fatty acids in milk from omega-3 fatty acid-supplemented mothers are related to less immunoglobulin E-associated disease in infancy. Acta Paediatr. 2016. 105:1337-1347. doi:10.1111/apa.13395.

\section{Rationale}

Outcome

\section{Statistical tests do not converge}

Study design Intervention/Exposure; Outcome; No Exp-outcome test 